\title{
CHARGED PARTICLE SPECTRA IN 32S + 32S INTERACTIONS AT 200 GEV/NUCLEON FROM CCD-IMAGED NUCLEAR COLLISIONS IN A STREAMER CHAMBER
}

\author{
LAWRENCE PAUL TEITELBAUM \\ Ph.D. Thesis \\ Physics Department \\ University of California
NUCLEAR SCIENCE DIVISION
Lawrence Berkeley Laboratory
University of California
Berkeley, CA 94720

APRIL 1992

\section{MASTER}

This work was supported by the Director, Office of Energy Research, Division of Nuclear Physics of the Office of High Energy and Nuclear Physics of the U.S. Department of Energy under Contract DE-AC03-76SF00098 


\title{
Charged Particle Spectra in ${ }^{32} \mathrm{~S}+{ }^{32} \mathrm{~S}$ Interactions at $200 \mathrm{GeV} /$ Nucleon from CCD-Imaged Nuclear Collisions in a Streamer Chamber
}

\author{
by \\ Lawrence Paul Teitelbaum \\ Ph.D. Thesis \\ April 1992 \\ Lawrence Berkeley Laboratory \\ University of California \\ Berkeley, California 94720
}

\begin{abstract}
We have measured the tiransverse momentum spectra $1 / p_{T} d N / d p_{T}$ and rapidity distributions $d N / d y$ of negatively charged hadrons and protons for central ${ }^{32} \mathrm{~S}+{ }^{32} \mathrm{~S}$ interactions at $200 \mathrm{GeV} /$ nucleon incident energy. The data were taken using the streamer chamber of experiment NA35 at the CERN SPS but with a novel imaging and data acquisition system based on charge-coupled devices (CCDs) as image sensors. The negative hadron $d N / d y$ distribution is too broad to be accounted for by thermal models which demand isotropic particle emission. It is compatible with models which emphasize longitudinal dynamics, by either a particle production mechanism, as in the Lund fragmentation model, or by introducing one-dimensional hydrodynamic expansion, as in the Landau model. The proton $d N / d y$ distribution, although showing no evidence for a peak in the target fragmentation region, exhibits limited nuclear stopping power. We estimate the mean rapidity shift of participant target protons to be $\Delta y \sim 1.5$, greater than observed for $p p$ collisions, less than measured in central $p A$ collisions, and much less than would be observed for a single equilibrated fireball at midrapidity. Both the negative hadron and proton $d N / d y$ distributions can be fit by a symmetric Landau two-fireball model. The negative hadron $p_{T}$ distribution is independent of rapidity, suggesting that the underlying invariant cross section factorizes, $E d^{3} \sigma / d^{3} p=f(y) g(k T)$. Although the spectrum possesses a two-component structure, a comparison to $p p$ data at comparable center-of-mass energy shows no evidence for enhanced production at low $p_{T} \lesssim 0.4 \mathrm{GeV} / c$. The two-component structure can be explained by a thermal and chemical equilibrium model which takes into account the kinematics of resonance decay. The proton $p_{T}$ distribution, although showing a much higher mean $p_{T}$ than in $p p$ data $\left\langle p_{T}\right\rangle \sim 0.600 \mathrm{GeV} / c$, is consistent with this model. Using an expression motivated by longitudinal expansion we find the same temperature for both the protons and negative hadrons at freezeout, $T_{f} \sim 170 \mathrm{MeV}$. We conclude that the charged particle spectra of negative hadrons and protons can be accommodated in a simple collision picture of limited nuclear stopping, evolution through a state of thermal equilibrium, followed by longitudinal hydrodynamic expansion until freezeout.
\end{abstract}




\section{Acknowledgements}

Numerous individuals have helped me in a multitude of ways to build the CCD Supervision System, participate in experiment NA35, analyze the data and write this dissertation.

First I must thank the members of the LBL streamer chamber group who were directly involved in the development of the CCD Supervision System. I would like to thank John Harris, the leader of the CCD group and my thesis adviser at LBL, for unfailing accessibility, encouragement to define my own vision for the project, and unflagging support, particularly during the endgame which dragged on far longer than I had anticipated. John's respectful and relaxed style of leadership also made it possible to balance the conflicting demands of my research and my family, which grew to include two children during my tenure with his group. Mark Tincknell presided over my early training and has remained a trusted friend. For two years, under constant pressure, Mark successfully wrestled with the design, construction and debugging of the interface board, and the development of the sensitive array processor machine code, the critical links in the data acquisition, without which there would have been no pictures, period. Wolfgang Rauch tutored me on a wide range of topics in statistics, mathematics, data acquisition and computing. Scott Chase, my fellow graduate student and office mate for the last five and one-half years, more than anyone else shared the daily struggle. Scott made important contributions to every facet of the project, from rewriting large pieces of system code to developing the computer measuring environment for the scanners to co-authoring the tracking code, and I benefited greatly from his broad knowledge of physics, mathematics and computing.

Howel Pugh, Grazyna Odyniec, Gulshan Rai and Shaheen Tonse all contributed greatly to my development as a physicist and helped me in different ways. Howel passed away before the data were mature but I frequently consulted his internal notes. Grazyna contributed her expertise in measurement of streamer chamber film, helped out with the optics during runs, and permitted her film scanners to measure our CCD pictures when we were just getting started. Grazyna was also a consistently supportive friend. Shaheen passed along a method for electron correction and taught me how to run the FRITIOF event simulation code. I am particularly grateful to Gulshan whom I turned to on a regular basis for answers to questions on statistics, error propagation, curve-fitting and minimization. I gratefully acknowledge the painstaking efforts of all the scanners who helped measure the data, Lois Tinay, Jean-Marie Walker, Youssef Billawalla, Hoover Wong, Santa Chatterji and Dipankar Ray. I would also like to thank T.J.M. Symons and the Nuclear Science Division of LBL for consistent support of this project.

I am grateful to Reinhard Stock and all the members of the NA35 Collaboration who welcomed us graciously to CERN. I must particularly thank Herbert Stroebele for quick responses to innumerable electronic mail inquiries, and for his continuing interest in my analysis as our data grew to maturity. I thank Rainer Renfordt for patience while we poked around in the dark stepping on his cameras, 
and for providing the run summaries and trigger cross sections. Peter Seyboth answered many questions about the momentum reconstruction and vertex fitting. Sigi Wenig was generous with details of his analysis of the film data. The image intensifier optical assemblies could not have been constructed without the advice and assistance of J.P. Fabre, K. Geissler and H. Fessler at CERN nor without the aid of $M$. Wensveen and Hugh Ellison who actually machined them. I was fortunate to have many useful discussions with Spyros Margetis who was a visitor at LBL while I was writing up the physics section of my thesis and who also contributed the calorimeter data and beam line figures.

I am indebted to Art Poskanzer and all the members of the Relativistic Nuclear Collisions Group at LBL for making building 50D such a fertile environment in which to do physics. In particular I would like to thank Peter Jacobs for useful discussions and general confidence building, and Erwin Friedlander for pointing me to the literature on double scanning, for suggesting the Monte Carlo method for error analysis, and for answering any and all questions on statistical methods. I would also like to thank Miklos Gyulassy of the Nuclear Theory Group for many informative discussions.

I also wish to thank P. Buford Price, my on-campus adviser at the University of California, Berkeley, and the other members of my thesis committee, Herbert Steiner, John Rasmussen, and Gerson Goldhaber. I regret that I did not consult with them more often.

It would have been impossible to complete this dissertation without the army of individuals who make LBL such a special community. I thank everyone at the Information and Computing Sciences Division, in particular, Marty Gelbaum, Wes Bethel, Nancy Johnston, and Ralph Dennis. Werner Koellner made it easy to set up the universe of CERN Library software on my own computer. I have never looked for a book or journal article and been unable to find it at the LBL library and I particularly thank Richard Robinson for help with literature searches.

Finally I thank my wife Barbara and my children Ben and Sophie. More than anyone, Barbara has shouldered the burdens associated with this quixotic undertaking. Her strength and faith never wavered(well, almost never) in the face of innumerable revisions of the projected completion date, more than a year of lost weekends, and the insidious domination which $m y$ thesis exercised over our life. Fortunately, I will have a long time to repay this enormous debt. During the final phase, five-year old Ben would remind Barbara to be patient, that "Daddy's working as hard as he can", and three-year old Sophie would ask me sweetly at dinner if I had completed my "PhD" that day. Although at first it seemed that it would be difficult to finish because of the children, it finally became clear that it would have been impossible to do it without their love and inspiration. 


\section{Contents}

$\begin{array}{ll}\text { Acknowledgements } & \text { ii }\end{array}$

Table of Contents $\quad$ iv

List of Figures vi

List of Tables $\quad$ xi

1 Introduction 1

1.1 Motivation . . . . . . . . . . . . . . . 1

1.2 Theoretical Considerations in

Nucleus-Nucleus Collisions . . . . . . . . . . . . 6

1.2.1 Dynamical Picture . . . . . . . . . . . . . 6

1.2 .2 Thermodynamics . . . . . . . . . . . . . 12

1.2.3 Hydrodynamic Expansion . . . . . . . . . . . . . 14

2 Experiment NA35 at the CERN SPS 17

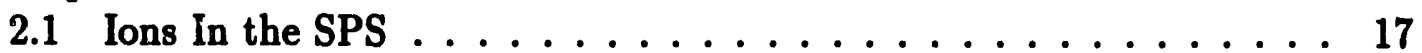

2.2 NA35 Experimental Overview . . . . . . . . . . . . 18

2.2.1 Streamer Chamber . . . . . . . . . . . 20

2.2 .2 Calorimetry . . . . . . . . . . . . . . 22

2.3 Trigger and Event Selection $\ldots \ldots \ldots \ldots \ldots \ldots$

3 CCD Supervision System 30

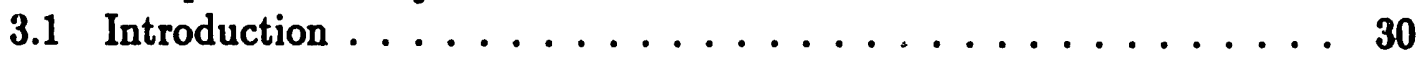

3.2 CCD Supervision System Hardware . . . . . . . . . . . . . 33

3.2.1 Charge-Coupled Devices . . . . . . . . . . . . . . 33

3.2.2 Cameras and Controllers . . . . . . . . . . . . . . . 39

3.2.3 Image Intensifier Optical Assemblies . . . . . . . . . 40

3.2.4 Host Computer and Array Processor . . . . . . . . . . . 44

3.2.5 Interface . . . . . . . . . . . . . . . 45

3.2.6 Graphics Display Station . . . . . . . . . . . . . . 46

3.2.7 Data Acquisition Cycle . . . . . . . . . . . . . . 46

3.3 Software . . . . . . . . . . . . . . . 47

3.4 Optical Configurations and Resolution $\ldots \ldots \ldots \ldots \ldots$

3.5 Online . . . . . . . . . . . . . . . 55 
4 Track Measuring, Track Matching and Reconstruction 58

4.1 Introduction . . . . . . . . . . . . . . . . . . 58

4.2 The Geometry of Photography and

Reconstruction ...................661

4.3 Optical Constants and Distortion Correction ............ 71

4.4 Track "Measuring" .................... 73

4.4.1 Tracking .................... 74

4.4.2 Track filtering .................. 77

4.4 .3 Cleanup ..................... 83

4.4.4 Scanning Efficiency ................. 86

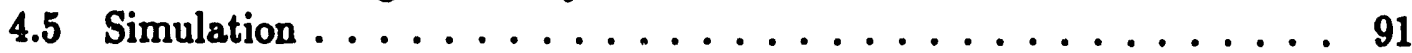

4.6 Track Matching . . . . . . . . . . . . . . . . 99

4.6.1 Projective Envelopes . . . . . . . . . . . . . . 99

4.6.2 Online Reconstruction ................ . 104

4.6.3 Ambiguity Resolution ................ 105

4.6.4 Performance on Scanner Matched Data . . . . . . . . . . . 106

4.7 Final Reconstruction and Vertex Fit .............. 107

4.8 Matching Efficiency and Spurious Track

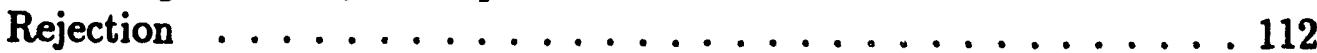

4.9 Momentum Resolution . . . . . . . . . . . . . . . 118

5 Corrections to the Data 123

5.1 Introduction ........................ 123

5.2 Geometrical Acceptance and Geometrical Weight . . . . . . . 124

5.3 Electron Correction . . . . . . . . . . . . . . . . . 134

5.4 Non-vertex Particles . . . . . . . . . . . . . . . . . 138

5.4.1 The simulation of $A A$ collisions including secondary

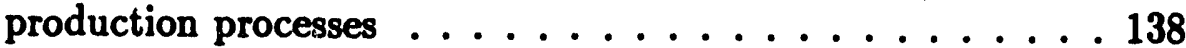

5.4.2 Corrections to the negative hadron $p_{T}$ and $d N / d y$ distributions ......................... 149

5.4.3 Corrections to the proton $d N / d y$ and $p_{T}$

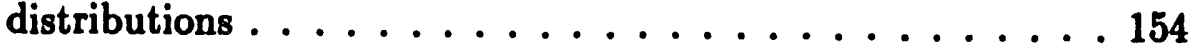

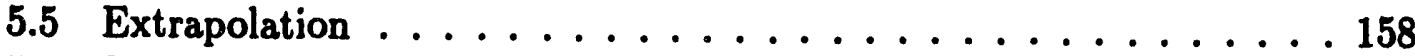

5.6 Summary of Corrections and Data Sample . . . . . . . . . . 162

6 Charged Particle Spectra 164

6.1 Rapidity Distributions ..................... 164

6.2 Transverse Momentum Spectra . . . . . . . . . . . 177

7 Summary and Conclusions $\quad 197$

$\begin{array}{ll}\text { Bibliography } & 200\end{array}$ 


\section{List of Figures}

1.1 Participant-spectator picture of a nucleus-nucleus collision at $p_{\text {inc }} \sim 1 \mathrm{GeV} / \mathrm{c} \ldots \ldots \ldots \ldots \ldots \ldots \ldots$

1.2 Ultrarelativistic nucleus-nucleus collision in the inside-outside cascade model . . . . . . . . . . . . . . 9

2.1 CERN machine complex employed for the acceleration of light ions 18

2.2 NA35 experimental apparatus $\ldots \ldots \ldots \ldots \ldots \ldots \ldots$

2.3 Schematic diagram of the NA35 streamer chamber . . . . . . . 21

2.4 Film image of a ${ }^{32} \mathrm{~S}+{ }^{32} \mathrm{~S}$ collision at $200 \mathrm{GeV} /$ nucleon $\ldots \ldots . .23$

2.5 NA35 beam counters $\ldots \ldots \ldots \ldots \ldots \ldots \ldots \ldots$

2.6 Differential cross sections $d \sigma / d E_{T}$ as a function of $E_{T}$ for $200 \mathrm{GeV} /$ nucleon ${ }^{32} \mathrm{~S}$ projectiles $\ldots \ldots \ldots \ldots \ldots$

$2.7 E_{T}-E_{V e t o}$ correlation $\ldots \ldots \ldots \ldots \ldots$

3.1 Directly digitized CCD image of a ${ }^{32} \mathrm{~S}+{ }^{32} \mathrm{~S}$ collision at 200 $\mathrm{GeV} /$ nucleon in the NA35 streamer chamber . . . . . . . . 31

3.2 CCD Supervision System hardware $\ldots \ldots \ldots \ldots \ldots \ldots$

3.3 Depth structure of Texas Instruments Virtual Phase CCD . . . . 35

3.4 Construction of two-dimensional imaging CCD . . . . . . . . 36

3.5 Two-stage image intensifier optical assembly . . . . . . . . . 41

3.6 Response curve of MCP image intensifier . . . . . . . . . 43

3.7 Camera positions $\ldots \ldots \ldots \ldots \ldots \ldots \ldots$ 
3.8 Normal magnification CCD image of an ${ }^{16} \mathrm{O}+$ Au collision at 200 $\mathrm{GeV} /$ nucleon $\ldots \ldots \ldots \ldots \ldots \ldots \ldots \ldots \ldots$

3.9 High magnification CCD image of an ${ }^{16} \mathrm{O}+\mathrm{Au}$ collision at 200 $\mathrm{GeV} /$ mucleon $\ldots \ldots \ldots \ldots \ldots \ldots \ldots \ldots$

3.10 Effective source size as a function of distance from the chamber midplane . . . . . . . . . . . . . . . . 54

3.11 Peak-finding along a single pixel contour $\ldots \ldots \ldots \ldots \ldots$

4.1 Square CCD image of a $\sim 1.2 \times 1.2 \mathrm{~m}^{2}$ section of the rectangular streamer chamber . . . . . . . . . . . . . . 60

4.2 Three spaces employed in measurement and reconstruction of visual tracking detector data $\ldots \ldots \ldots \ldots \ldots \ldots$

4.3 Standard parametrization of a charged particle trajectory . . . . . 65

4.4 Kinematic relations between rightside and wrongside tracks . . . . 66

4.5 Camera-to-camera mappings . . . . . . . . . . . . 70

4.6 Peak-finding along elliptical contours $\ldots \ldots \ldots \ldots \ldots \ldots$

4.7 Deviations of measured track points from circle fits $\ldots \ldots \ldots$. . 78

4.8 Number of measured points as a function of projected arc length . . 80

4.9 Rms deviation of circle fits before and after iterative filter $\ldots \ldots 80$

4.10 Definition of fractional projected arc length $f p l \ldots \ldots \ldots$. . . . 81

4.11 Maximum projected arc length distribution for ideally measured tracks 82

4.12 Fractional projected arc length $f p l$ as a function of $\theta_{\text {proj }} \ldots \ldots 84$

4.13 Chromosome measuring defect $\ldots \ldots \ldots \ldots$. . . . . . . 87

4.14 Scanning efficiency. Composite event of all tracks in the category seen by $A(B)$ but not by $B(A) \ldots \ldots \ldots \ldots . \ldots . \ldots 89$

4.15 Magnetic field profiles $\ldots \ldots \ldots \ldots \ldots \ldots$ 
$4.16 \mathrm{Rms}$ deviation of track fits in the film plane $\ldots \ldots \ldots \ldots$

4.17 Projected distance to the start point $\ldots \ldots \ldots \ldots \ldots$

4.18 Influence of central cone on rapidity density of negative hadrons from simulated data $\ldots \ldots \ldots \ldots \ldots \ldots \ldots$

$4.19 \psi$ distributions for positively and negatively charged particles from simulated data $\ldots \ldots \ldots \ldots \ldots \ldots$

4.20 Chamber-boundary projective envelope . . . . . . . . . . . 101

4.21 Dip plane-boundary projective envelope . . . . . . . . . 103

4.22 Geometry of the vertex fit $\ldots \ldots \ldots \ldots \ldots$

4.23 Vertex fit distributions $\ldots \ldots \ldots \ldots \ldots \ldots \ldots$

$4.24 \chi_{v t x}^{2}$ distribution for reconstructed simulated tracks $\ldots \ldots \ldots 111$

$4.25 \chi_{v t x}^{2}$ distribution for measured data $\ldots \ldots \ldots \ldots \ldots \ldots$

4.26 Differences in transverse momentum $p_{T}$ and rapidity $y$ for compromised tracks . . . . . . . . . . . . . . . . 114

4.27 Matching efficiency $\eta_{\text {match }} \ldots \ldots \ldots \ldots \ldots \ldots$

4.28 Spurious track contamination $\eta_{\text {spur }} \ldots \ldots \ldots \ldots \ldots$

4.29 Comparison of the rapidity density $d N / d y$ for spurious tracks and correctly matched tracks . . . . . . . . . . . . . 117

$4.30 \chi_{v t x}^{2}$ distribution for Monte Carlo data processed through the complete data reduction chain . . . . . . . . . . . . 118

4.31 Pull quantities for the momentum fit parameters $1 / p, \lambda, \phi \ldots \ldots 120$

4.32 Momentum resolution $\delta p / p$ as a function of $p \ldots \ldots \ldots 121$

4.33 Transverse momentum resolution $\delta p_{T} \ldots \ldots \ldots \ldots$. . . . . 122

5.1 Geometrical $\left(y, p_{T}\right)$ acceptance for $\pi^{-} \ldots \ldots \ldots \ldots$

5.2 Geometrical $\left(y, p_{T}\right)$ acceptance for protons. . . . . . . . 127 
5.3 Projected arc length distributions splmax as a function of $\psi$ for selected values of $\left(p, \theta_{l a b}\right) \ldots \ldots \ldots \ldots \ldots \ldots \ldots$

5.4 Comparison of inverted, "measured" distribution to the FRITIOF reference spectrum. . . . . . . . . . . . . . . . 132

5.5 Final pion and proton acceptance in $\left(y, p_{T}\right)$ space. . . . . . . 133

5.6 Normalized electron and pion rapidity distributions $\ldots \ldots \ldots . \ldots 137$

5.7 Ratio of the electron yield to the negative hadron yield as a function

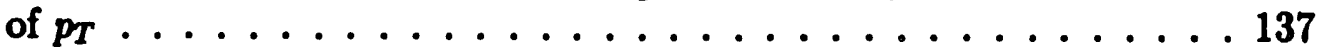

5.8 Representative elements of the electron correction table $c_{i j}\left(y, p_{T}\right) \quad .138$

5.9 Number of charged particles from weak decay and secondary hadronic interaction as a function of secondary vertex position . . . 143

5.10 Bend-plane trajectories of charged secondaries for one event $\ldots \ldots 144$

5.11 Number of negative hadrons as a function of $\left(y, p_{T}\right)$ for particles originating in primary, decay and secondary hadronic processes . . 145

5.12 Bend-plane trajectories of secondaries produced in hadronic interactions . . . . . . . . . . . . . . . . 147

5.13 Contributions of primary, weak and secondary hadronic processes to the negative hadron transverse momentum distribution $\ldots \ldots 149$

5.14 Comparison of negative hadron $d N / d p_{T}$ spectra of measured data and the full Monte Carlo . . . . . . . . . . . . . 150

5.15 Ratio of the sum of the primary, weak and secondary hadronic components to the primary spectrum as a function of $p_{T} \ldots \ldots \ldots 152$

5.16 Corr ion factors to the negative hadron $p_{T}$ spectrum $\ldots \ldots 152$

5.17 Rapidity dependence of the correction to the negative hadron

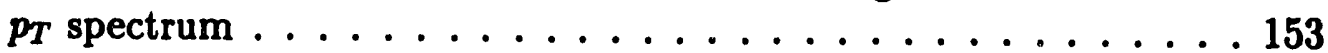

5.18 Correction factors to the negative hadron $d N / d y$ distribution $\ldots \ldots 153$

5.19 Contributions to the the charge-excess distribution $d N / d y \ldots \ldots$ 
5.20 Transverse momentum dependence of $d N / d p_{T}$ from $\left(h^{+}-h^{-}\right)_{\text {weak }}$ for different rapidity intervals $\ldots \ldots \ldots \ldots \ldots \ldots$

5.21 Ratios and correction factors for the proton $d N / d y$ distribution $\ldots 157$

5.22 Ratios and correction factors for the proton $p_{T}$ distribution $\ldots \ldots 158$

5.23 Transverse mass $m_{T}$ fit to the negative hadron $p_{T}$ spectrum for extrapolation down to $p_{T}=0 \ldots \ldots \ldots \ldots \ldots \ldots$

5.24 Transverse mass $m_{T}$ fit to the proton $p_{T}$ spectrum $\ldots \ldots \ldots 159$

5.25 Monte Carlo extrapolation of proton $d N / d y$ distribution $\ldots \ldots 161$

6.1 Fully corrected negative hadron $d N / d y$ distribution $\ldots \ldots \ldots \ldots 165$

6.2 Landau fireball fits to the negative hadron $d N / d y$ distribution $\ldots 168$

6.3 Corrected proton $d N / d y$ distribution $\ldots \ldots \ldots \ldots \ldots$

6.4 Landau two-fireball fit to the proton $d N / d y$ distribution $\ldots \ldots 172$

6.5 Fully corrected negative hadron $p_{T}$ distributions $1 / p_{T} d N / d p_{T} \ldots 179$

6.6 Single-component thermal fits to negative hadron $p_{T}$ distributions . 181

6.7 Linear plus quadratic exponential fit to negative hadron $p_{T}$ distributions. Ratio of $A A$ to $p p \ldots \ldots \ldots \ldots \ldots \ldots$

6.8 Two-component thermal fits to negative hadron $p_{T}$ distributions $\ldots 188$

6.9 Proton transverse rnomentum distribution $\ldots \ldots \ldots \ldots$ 


\section{List of Tables}

3.1 Characteristics of first and second stage image intensifier tubes . . . 42

3.2 Characteristics of primary and relay lenses at high and normal magnification ..................... 50

4.1 Calculation of partition-dependent scanning efficiency . . . . . . 90

5.1 Default and modified FRITIOF/JETSET parameters . . . . . 139

5.2 Material parameters important for secondary hadronic interactions

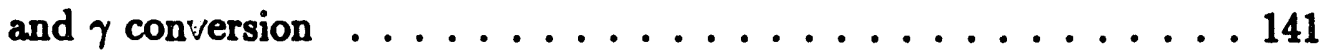

5.3 Contributions to observable $d N / d y$ charge-excess distribution . . . 154

5.4 Monte Carlo extrapolation factors for the proton $d N / d y$ distribution 161

5.5 Influence of selection cuts on data sample . . . . . . . . . 163

6.1 Summary of the fits to proton and negative hadron $d N / d y$

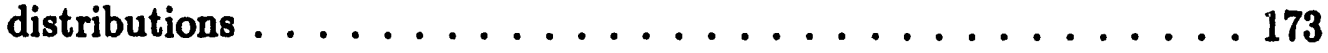

6.2 Summary of two-component fits to the negative hadron

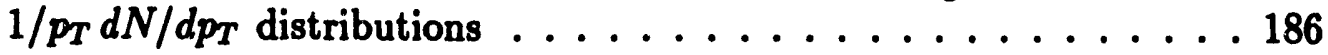

6.3 Summary of single-component fits to the proton $1 / p_{T} d N / d p_{T}$

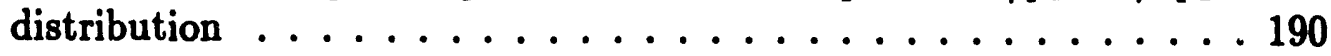




\section{Chapter 1}

\section{Introduction}

\subsection{Motivation}

The main motivation for investigating relativistic and ultrarelativistic nucleusnucleus collisions is to produce and study strongly interacting matter under extreme conditions of high particle density, high energy density, and high temperature. In the relativistic domain, at incident momentum per nucleon $p \sim 1 \mathrm{GeV} / c$, the emphasis of research is on understanding the nuclear matter equation of state at high baryon density. For incident particle velocities greater than the speed of sound, the development of a shock wave might lead to baryon densities 3-5 times greater than in normal nuclear matter [Sch74]. In this regime the nucleon degrees of freedom dominate. Particle production occurs mainly through the excitation and decay of baryon resonances. In the ultrarelativistic domain, $p \gtrsim 10 \mathrm{GeV} / c$, temperatures and energy densities are expected to be higher, baryon densities may be significantly lower, and the subnucleon constituents-quarks and partons-become important. As the center-of-mass energy $\sqrt{3}$ rises, multiparticle production processes dominate the inelastic cross section. In this regime the focus of experimentation is the detection of quark matter, the quark gluon plasma predicted by quantum chromodynamics (QCD), the gauge field theory of the strong interaction. The subject of this thesis is a measurement of the charged particle spectra resulting from collisions of ${ }^{32} \mathrm{~S}$ nuclei at $200 \mathrm{GeV} /$ nucleon bombarding energy at the Super Proton Synchrotron (SPS) accelerator at CERN, the European Laboratory for Particle Physics. Corresponding to $\sqrt{s}=20 \mathrm{GeV}$, this is the highest energy range yet explored with nuclear projectiles more massive than $\alpha$ particles. It will remain so until the first generation of experiments at RHIC, the relativistic heavy-ion collider now under construction.

Soon after the introduction of the quark model by Gell-Mann [Gel64] to explain the spectroscopy of hadrons, speculations were entertained that dense stars were composed of quark matter [Ito70]. The existence of a quark gluon plasma is a natural consequence of the asymptotic freedom property of QCD. Collins and Perry [Col75] were perhaps the first to apply QCD and specifically invoke asymptotic freedom to argue that at densities much higher than nuclear-matter density, strongly interacting matter should consist of locally-free quarks. Study of the pro- 
duction and evolution of the quark gluon plasma and its subsequent decay into hadronic matter might provide insight into confinement, the phenomenon which accounts for the non-observation of free quarks and gluons. Much of the motivation for colliding heavy nuclei, beyond the excitement of searching for a new phase of matter, is the prospect of addressing fundamental questions in QCD that are difficult to answer in the perturbative regime of hard parton-parton scattering.

Why nucleus-nucleus collisions? First, the energy density $\varepsilon$ is likely to be higher in $A A$ than in $p p$ collisions at the same available center-of-mass energy $\sqrt{s}$, because of the large number of produced particles. If the multiplicity increases like the nuclear mass $A$ while the average energy per particle remains the same, and if the interaction volume is cylindrical because of Lorentz contraction along the beam direction so that $V=\pi R^{2} \Delta L$ where $\Delta L \sim 1 \mathrm{fm}$ is an energy-independent limiting nuclear thickness [McL82] and the nuclear radius $R \sim A^{1 / 3}$, then

$$
\varepsilon=E / V \sim A / A^{2 / 3} \sim A^{1 / 3} \text {. }
$$

For Au nuclei, $A=197$, the energy density may be six times higher than in $p p$ collisions at the same $\sqrt{s}$. Second, the transverse dimensions of nuclei are urge compared to typical hadronic mean free paths. For example, the mean free path of a proton in normal nuclear matter would be

$$
\lambda=1 /(\mathrm{n} \sigma) \sim 1 /\left(.15 \mathrm{fm}^{-3}\right)(40 \mathrm{mb}) \sim 1.7 \mathrm{fm}
$$

$\lambda$ would be much smaller for Lorentz-contracted heavy nuclei. This means that particles produced in nucleus-nucleus collisions would rescatter many times, enabling them to come to thermal equilibrium.

High energy nucleus-nucleus collisions are generally believed to take place in three stages: a fast interpenetration and compression stage, a high density stage which may be long enough for the excited matter to reach thermal equilibriumto achieve a thermodynamic state-thereby justifying application of the concepts and methods of statistical physics, followed by an expansion until freezeout, the time when nuclear matter becomes too dilute to undergo further interaction and final-state particles stream freely toward detectors. What we hope to measure are signals which are generated during the high-density stage. This is presumably when quark matter is produced. Observables which originate during this stage and which escape the system with only weak subsequent interaction are called penetrating probes. They bring information out from the plasma. Primordial observables reach their asymptotic values during the high-density stage in spite of non-negligible interaction during evolution. Freezeout observables are strongly influenced by the expansion dynamics and assume their values as particles decouple from the interaction region. Charged particle spectra may be able to address all three stages of a nucleus-nucleus collision.

In this thesis we will present distributions for the single-particle inclusive process

$$
a+b \rightarrow c+X
$$


where $a$ and $b$ are the incident projectile and target, $c$ is a particula- final-state particle, and $X$ stands for whatever else may be produced along with $c$. A fully inclusive measurement selects essentially all events in which $c$ occurs, with a minimum of experimental bias, only requiring that an interaction has taken place. A semi-inclusive measurement is more restrictive, imposing additional criteria for event selection, for example by requiring that $X$ consists of at least $n$ charged particles. In nucleus-nucleus collisions, since we are interested in events with large energy densities, large numbers of particles and large volumes, we try to select central collisions in which the nuclei completely overlap and most of the constituent nucleons participate in inelastic interactions. The triggers that were used to select central events in a relatively unbiased manner will be discussed in Chapter 2.

The kinematic variables we will employ are the transverse momentum $p_{T}$ and the longitudinal rapidity $y$,

$$
\begin{gathered}
p_{T}=\sqrt{p_{y}^{2}+p_{z}^{2}} \\
y=\frac{1}{2} \ln \frac{E+p_{L}}{E-p_{L}},
\end{gathered}
$$

where $p_{L}=p_{x}$ is the component of momentum along the beam direction and $E$ is the energy. The main reason for using $y$ to describe longitudinal motion is the way it transforms under Lorentz boosts,

$$
y \rightarrow y^{\prime}=y+\ln \gamma(1+\beta),
$$

where $\gamma$ and $\beta$ are the usual Lorentz transformation variables. Equation 1.4 implies that differences in rapidity are Lorentz-invariant and that the rapidity distribution, the particle density per unit of rapidity $d N / d y$, has the same shape in any reference frame. We have measured the single-particle inclusive rapidity and transverse momentum distributions for leading protons and negative hadrons $\left(h^{-}\right)$in central collisions of ${ }^{32} \mathrm{~S}$ projectiles with ${ }^{32} \mathrm{~S}$ targets. The transverse momentum distribution $1 / p_{T}\left(d N / d p_{T}\right)$ is related to the invariant cross section by an integration over rapidity,

$$
E \frac{d^{3} \sigma}{d p^{3}}=\frac{1}{\pi} \frac{d^{2} \sigma}{d y d p_{T}^{2}} \rightarrow \frac{1}{p_{T}} \frac{d N}{d p_{T}} .
$$

The proton rapidity distribution may bring out information from the interpenetration stage: how many binary nucleon-nucleon collisions have occurred and how much energy has been deposited when a fast baryon traverses nuclear matter [Bus88]. The average baryon rapidity loss $\langle\Delta y\rangle$ has been used as a measure of the nuclear stopping power in high-energy hadron-nucleus and light $A A$ collisions both in experimental [Too87], [Abe88], [Bel86] and theoretical studies [Dat85], [Bow86]. From the proton $d N / d y$ distribution we can estimate the baryon density which may be achieved in central collisions of heavy nuclei, which determines the character, baryon-rich or baryon-free, of the quark matter which we hope to produce in the collisions. The negative hadron $d N / d y$ distribution addresses multiparticle production: does it occur mainly by a central mechanism or by a fragmentation process in which projectile and target nucleons are excited 
to higher mass states which subsequently decay [Whi74], and does cascading in nuclear matter make a significant contribution [DeM82a]? The rapidity distribution of produced particles also addresses the degree of thermalization which may have been achieved and the influence of the expansion dynamics on the freezeout observables [Sta89b], [Lee90]. It also may provide a good estimate of the energy density, especially if the expansion is governed by scaling hydrodynamics [Gyu84]. The rapidity distributions of produced particles and baryons may be primordial. For the case of boost-invariant hydrodynansic expansion [Bj083] the particle density per unit of rapidity is a constant of the motion and carries out information from the high-density stage.

The transverse momentum distributions may measure the temperature of hot nuclear matter. The Fermi-Dirac, Bose-Einstein, and Maxwell-Boltzmann statistical distributions all make definite predictions for the shape of the $p_{T}$ spectra for a system in thermal equilibrium at temperature $T$. Hagedorn has argued [Hag83] that no experimental evidence from $p p$ interactions at any energy scale up to $\sqrt{s} \sim 50 \mathrm{GeV}$ contradicts the assertion of all statistical models that the inclusive $p_{T}$ distribution should be approximately thermal for $p_{T} \lesssim 1-1.5 \mathrm{GeV} / c$. Temperatures are determined at freezeout. In any realistic model the system cools during expansion-there is no thermal reservoir to maintain the temperature. Collective effects during expansion can modify the shape of an initial thermal spectrum [Sie79], [Lee89], which suggests that the transverse momentum distribution may help clarify the expansion dynamics. The $p_{T}$ distribution can also address whether chemical equilibrium, which determines the relative abundance of different stable particles and resonances, has been reached and whether it has been sustained during expansion. Its shape is sensitive to the kinematics of resonance decay [Bro91], [Sol90], and so indirectly to the abundances, and also to the loss of chemical equilibrium during expansion [Kat90], [Ger90]. Because $p_{T}$ distributions have been extensively measured in $p p$ collisions we can compare $A A$ to $p p$ results to search for departures from linear superposition of $p p$ physics.

The measurements presented in this thesis have been made as a member of the NA35 collaboration. Experiment NA35 employs calorimetry for triggering and energy flow studies [Bac91] but its unique feature is a large volume streamer chamber, a relatively fast, triggerable, visual tracking detector which can measure the momenta of nearly all charged particles from the collision over broad ranges of the kinematic variables $y$ and $p_{T}$. Visual techniques have always played an important role in particle physics, even in the age of electronic detectors. Bubble chambers and streamer chambers have been utilized as survey instruments in exploratory experiments, particularly in strong interaction physics as new energy scales have opened with the development of more powerful particle accelerators [Whi74], [Aln87]. This was the role envisioned for NA35 [GSI85]. The streamer chamber is able to measure charged particle multiplicities, single particle inclusive distributions for processes with large cross sections, multiparticle correlations, and neutral strange particle $\left(\Lambda, \bar{\Lambda}, K_{S}^{0}\right)$ yields and spectra, all with a minimum of experimental bias. Its two main disadvantages are its intrinsically slow data reduction rate-film data is conventionally measured by hand-and its inability to 
directly identify the mass of high-energy charged particles. A problem specific to NA35 is that in a fixed-target experiment the center-of-mass is located at forward laboratory angles, concentrating half the charged particles at polar angles $\theta_{\text {lab }}<5^{\circ}$. With greater than 200 charged particles in central collisions, the spatial resolution of the streamer chamber is often exceeded, significantly reducing the traditional $4 \pi$ solid angle acceptance of the detector. For ${ }^{32} \mathrm{~S}$ projectiles, this has made it difficult to measure data for heavy nuclear targets. Consequently, we have been unable to study the spectra systematically as a function of target mass. However, NA35 has obtained a relatively complete picture of an average central $S+S$ event from the $p_{T}$ and rapidity distributions of protons, $h^{-}, \Lambda, \pi$, and $K_{S}^{0}$ [Wen90].

A significant fraction of this thesis project was devoted to the development and implementation of a new imaging and data acquisition system using chargecoupled devices (CCDs) as image sensors in order to take direct digital images of streamer chamber events. The long-term goal was the metamorphosis of the streamer chamber into a completely electronic detector with particle identification capability. We have only partially succeeded. We have demonstrated the feasibility and desirability of this method, accomplished an acceleration of the data reduction rate, and obtained the only measurement of the proton $p_{T}$ and rapidity distributions presently available from nucleus-nucleus collisions at $200 \mathrm{GeV} / c$, other than that of our NA35 colleagues from the analysis of film data with conventional techniques. The development of large-scale time-projection chambers (TPCs) for nucleus-nucleus collisions [Pug86], [Kad90], [Lov91] probably signals the demise of the streamer chamber as an important detector. No second generation version of our system is likely to be constructed.

The organization of this thesis is as follows. In the remainder of this chapter we briefly present some of the theoretical considerations relevant to nucleus-nucleus collisions. Because the subject is interdisciplinary, incorporating elements from nuclear physics, particle physics, thermodynamics, statistical mechanics, and hydrodynamics, the theory is vast. Our objective is to develop a clearer intuitive picture of the collision dynamics, and introduce pertinent concepts and results from thermodynamics and hydrodynamics since they have played leading roles in calculations and theoretical development. Chapter 2 describes experiment NA35 at the CERN SPS. Chapter 3 discusses the system we have built for imaging and data acquisition, the CCD Supervision System. Readers uninterested in technical details may choose to omit this chapter although we encourage them to glance at the beautiful streamer chamber images. Chapter 4 is very long. Because the imaging hardware was new and a completely new data reduction machinery was constructed, we decided to carefully document our methods. We elected to present the material in a linear and self-contained fashion. The most important sections for the general reader: Section 4.4.2 and 4.4.4 where we develop a track measurement model that accounts for the influence of imperfect measuring on the data and parametrize the model from the measured data; Section 4.5 where we discuss simulation and introduce concepts we use later to define our acceptance; Section 4.7 because we show a number of important plots; and Section 4.8 where we estimate the efficiency of the full data reduction chain. Chapter 5 is important. One of the 
difficulties in comparing $A A$ data from different experiments or comparing it to earlier $p p$ and $p A$ results is the inconsistency between the corrections which have been applied to the data. In particular, a tremendous amount of theoretical activity was driven by two preliminary results which reported a strong enhancement, relative to $p p$ data, of the pion $p_{T}$ spectrum at low $p_{T}$. Chapter 6 presents and discusses our results, the transverse momentum and rapidity distributions of protons and negative hadrons, compares some of their features to results from $p p, p A$, and light $A A$ experiments at the same energy, and interprets the spectra in the spirit of several models. Chapter 7 is a brief summary. The main conclusion of this thesis is that the spectral features of both the leading protons and the produced hadrons can be accounted for by a simple model based on limited nuclear stopping, evolution to a state of thermal equilibrium, followed by longitudinal, hydrodynamic expansion. We obtain estimates for the temperature $T$, the baryon density $n_{b}$, and the energy density $\varepsilon$ at an early stage of the collision. Our results suggest that the deconfinement phase transition is within reach at SPS energy and that quark matter is likely to be baryon-rich for the heavy nuclear projectiles which will become available in the next generation of experiments at CERN.

\subsection{Theoretical Considerations in Nucleus-Nucleus Collisions}

\subsubsection{Dynamical Picture}

What happens when two nuclei collide at impact parameter $b$ ? Figure 1.1 illustrates the geometry of a nucleus-nucleus collision at relativistic incident laboratory momentum $p_{\text {inc }} \sim 1 \mathrm{GeV} / c$ in the center-of-mass frame. At this momentum, the Lorentz $\gamma$ factor of each nucleon, measured with respect to the center-of-mass, is $\gamma=1.1$ so Lorentz contraction is negligible. At energies much higher than the nuclear binding energy, the main role of intranuclear forces is to confine nucleons in the nucleus with internal momenta distributed according to their Fermi motion up to a maximum $p_{F} \sim 270 \mathrm{MeV} / c$. Since the range of nuclear forces is $\sim 1 \mathrm{fm}$, the size of a nucleon, only particles in the overlap region have a significant probability of interacting strongly-they are the participants in the reaction, the nucleons which do not overlap are spectators to the collision. In the simple fireball model [Wes76], which assumes clean cut geometry and treats nuclei as hard spheres, the available energy heats participant matter to a temperature $T$ estimated from relativistic ideal gas thermodynamics. The fireball, at rest in the center-of-mass $y_{\mathrm{cm}}=0$, decays isotropically. The center-of-mass rapidity is referred to as midrapidity because

$y_{\mathrm{cm}}=0$ at $1 / 2\left(y_{\text {proj }}-y_{\mathrm{targ}}\right)$ for collisions of equal-mass nuclei [Sto86], independent of impact parameter. Spectators may introduce distortions either by directly contaminating participant matter observables or because participants undergo reinteraction in spectator matter. Both effects will be diminished in central collisions of equal-mass nuclei, especially at midrapidity. In asymmetric collisions, where the mass/size of the target is much greater than that of the projectile, spectator effects 


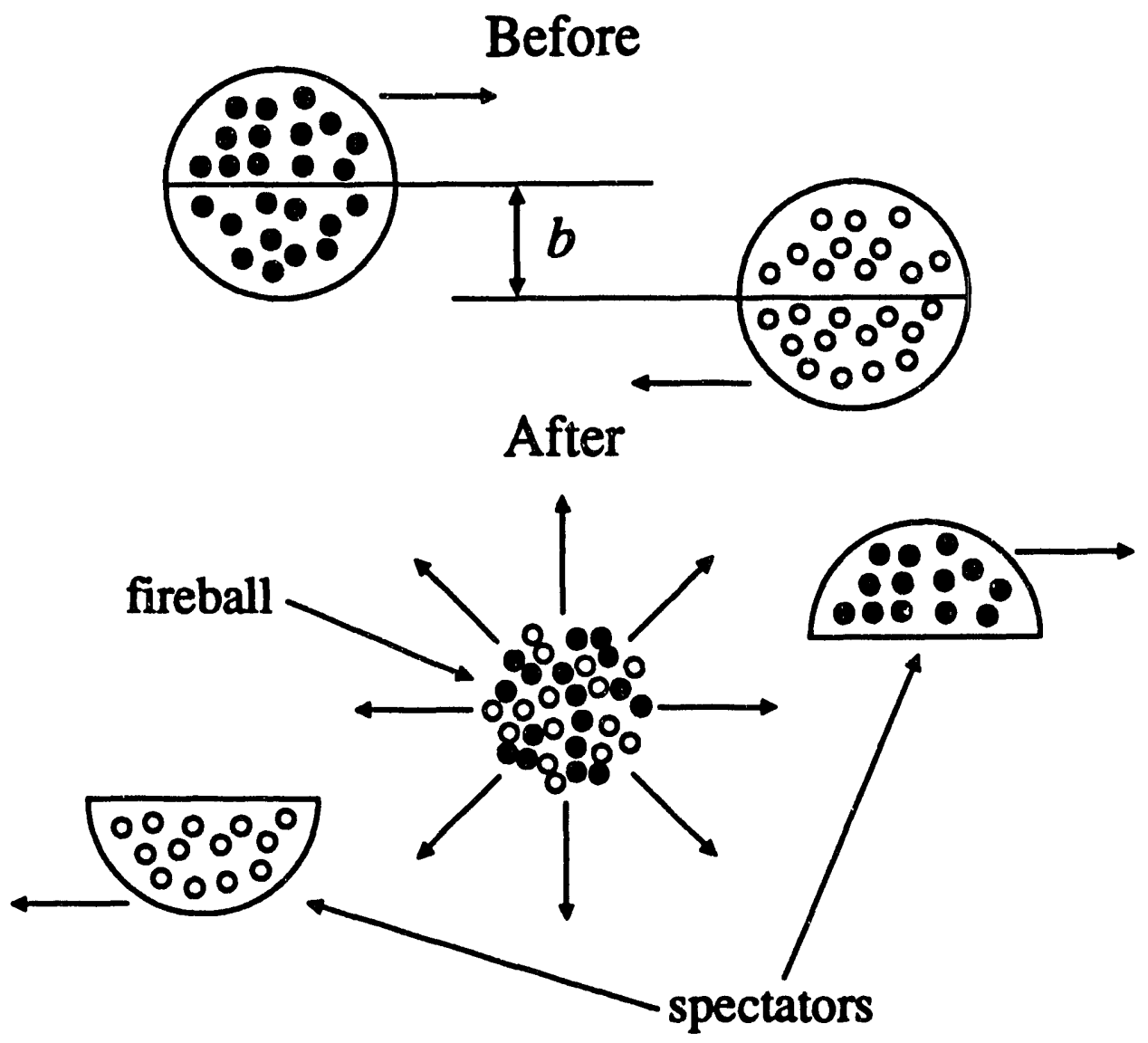

Figure 1.1: Participant-spectator picture of a nucleus-nucleus collision at $p_{\text {inc }} \sim 1$ $\mathrm{GeV} / c$. Lorentz contraction is negligible. Participant matter is fully stopped and, in the simplest model, forms a thermal fireball which decays isotropically. The spectators stream forward, essentially at the velocity of the projectiles, with a dispersion in velocity which can be ascribed to Fermi motion [Gol74]. 
will be especially strong, particularly in the target rapidity hemisphere $y<y_{\mathrm{cm}}$. In asymmetric collisions there is always a large volume of spectator matter.

As the colliding nuclei begin to interpenetrate, binary nucleon-nucleon collisions occur. Interacting nucleons acquire transverse momentum and are excited to resonant states. The effectiveness of nuclear matter at redistributing the initial longitudinal momentum into baryon resonance production, particle production, and transverse degrees of freedom is called the nuclear matter stopping power. As the first participating nucleons are slowed by collisions, the trailing nucleons continue to stream forward at their initial velocity. This dynamical pileup [Sto86], denoted shock compression because it is predicted from hydrodynamical calculations [Sch74] to occur when the initial interpenetration velocity is greater than the nuclear sound speed, leads to baryon densities which exceed the superposition density $2 \gamma \rho_{0}$ where $\rho_{0}$ is the density of normal nuclear matter. Since the initial velocity in the center-of-mass is $\beta \sim 0.5$, the timescale for the interpenetration phase is

$$
\tau=\frac{\text { distance }}{\text { velocity }} \sim \frac{2 R}{\gamma \beta} \sim 20 \mathrm{fm} / c
$$

where $R \gtrsim 5$ is the radius of a heavy nucleus at rest. Because heavy nuclear targets present many mean free paths there is both sufficient time and sufficient material to ensure that each incident nucleon undergoes many binary $N N$ collisions. If the available energy is equipartitioned among the different degrees of freedom the nucleus-nucleus system is said to be fully stopped. For the case of complete stopping, participants are brought to rest in the center-of-mass, high baryon densities are achieved by shock compression, and the mechanism for equilibration is multiple, binary $N N$ collisions. The lifetime of the high density stage, estimated from cascade calculations to be $\sim 5-10 \mathrm{fm} / c$ [Har84a], is long enough for elastic and inelastic collisions to lead to thermal and chemical equilibrium. Neither multiparticle production nor the parton substructure enters into the description. The high density stage is dominated by nucleons and baryon resonances.

If the fireball were to decouple instantaneously at the end of the high density stage, without subsequent interaction, all resonances would decay and the remaining stable particles would stream freely to detectors. For a given temperature, particle yields would be determined from the open resonance channels and particle spectra from the superposition of resonance decay kinematics onto thermal distributions. Instead, the expansion dynamics may introduce significant perturbations. During expansion, the system cools and the particle density decreases, increasing the mean free path. Collisions become less frequent. Depending on the timescale of the expansion, the fireball may fall out of chemical and/or thermal equilibrium [Har84b]. Different observables may freeze out at different times [Nag82]. At $p_{\text {inc }} \sim 1 \mathrm{GeV} / c$, because 4-5 average $N N$ collisions during interpenetration should be sufficient to equilibrate the longitudinal and transverse degrees of freedom, the expansion is often assumed to be spherically symmetric [Bon78], [Sie79].

The situation at ultrarelativistic momentum $p_{\text {inc }} \sim 200 \mathrm{GeV} / c$ is shown in Figure 1.2 for $a b=0$ collision. The dynamical picture changes dramatically at this energy scale. The Lorentz $\gamma$ factor is $\gamma=10$, the nuclei approach each other 

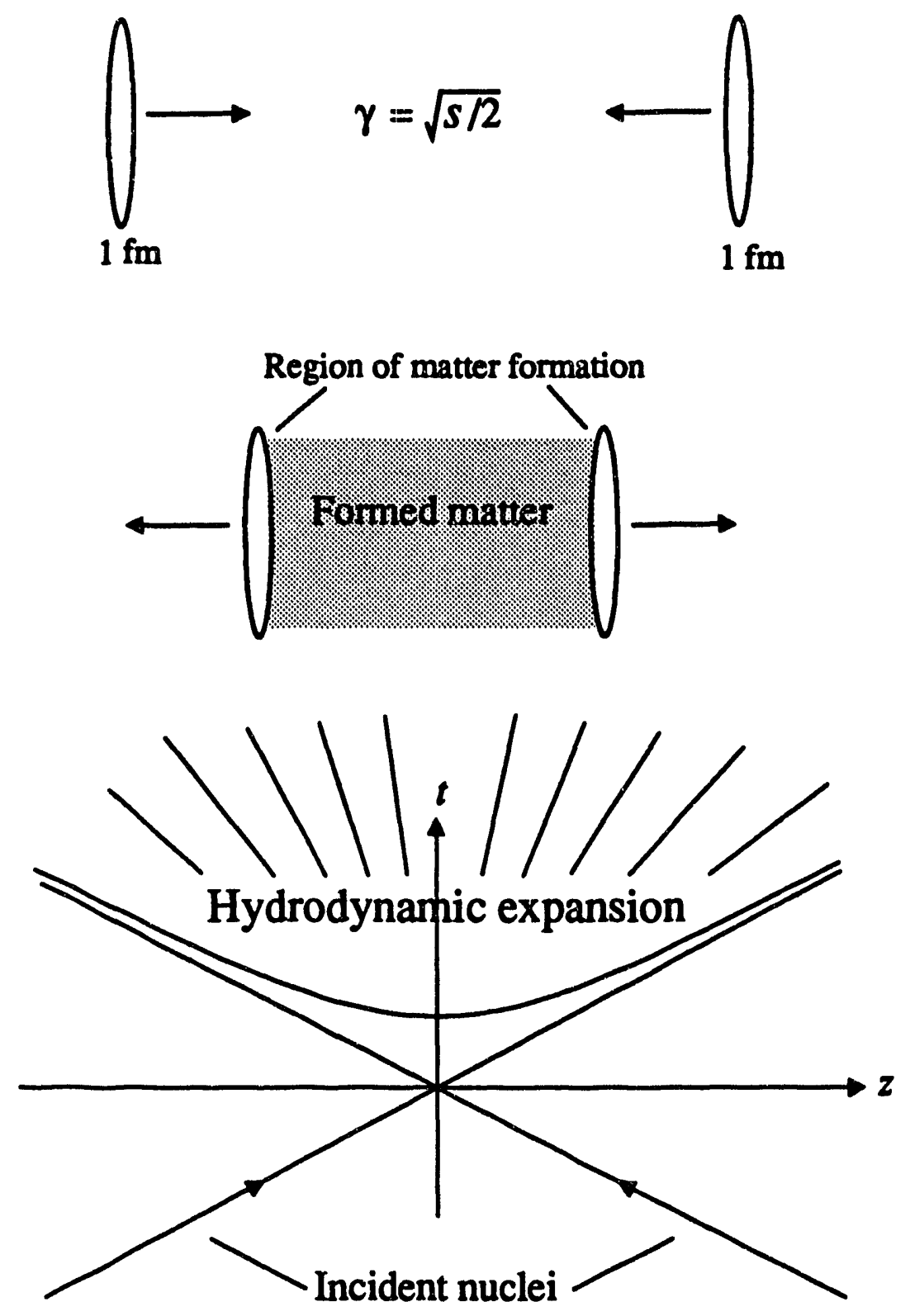

L

Figure 1.2: Ultrarelativistic nucleus-nucleus collision in the inside-outside cascade model. For $\sqrt{s} \gtrsim 20 \mathrm{GeV}$ the nuclei are Lorentz contracted to $\sim 1 \mathrm{fm}$. Baryon number is confined to the fragmentation regions. Matter forms at the proper formation time on the space-time hyperbola $\tau_{0}^{2}=t^{2}-z^{2}$. The initial condition for hydrodynamic expansion is $v_{z}=z / t$ where the expansion begins at later times and larger velocities further from the collision point. 
at $\beta=1$, and they are contracted to a limiting thickness $\sim 1 \mathrm{fm}$ [Bjo76]. The timescale for interpenetration is short and independent of nuclear size

$$
\tau=\frac{\text { distance }}{\text { velocity }} \sim \frac{1 \mathrm{fm}}{\beta} \sim 1 \mathrm{fm} / c .
$$

Although the low-momentum components of tha nuclear wavefunction may interact strongly [Bjo76], [McL82], producing particles near $y_{c m}=0$, the valence constituents pass through each other and are not significantly slowed by the collision, that is, the nuclei are transparent [Fer79]. Transparency means that in $p A$ and $A A$ collisions the leading baryon(s), the fast baryon(s) which intuitively can be associated with the incident nucleon(s), and fast fragments are distributed ás if they had undergone a single nucleon-nucleon collision [Ani80]. It is explained by postulating the existence of a proper formation time $\tau_{0} \sim 1 \mathrm{fm} / c$, the proper time required to form a secondary hadron [Gyu84], [Kaj82]. Because of Lorentz time dilation, in any other frame the formation time increases,

$$
t(y)=\gamma(y) \tau_{0}=\tau_{0} \cosh y .
$$

This phenomenon is also known as longitudinal growth-the distance scale of the interaction grows with rapidity and fast projectile fragments are produced far outside the target [Nik81]. Because the interpenetration timescale is $\simeq \tau_{0}$, collisions at this energy cannot evolve according to a semiclassical $N N$ cascade among hadrons in well defined states. Calculations typically assume that multiple excitations occur during the short traversal time followed by fragmentation which depends on the number of nucleon-nucleon collisions [Dat85], [Bow86]. Leading baryon $d N / d y$ distributions from $p A$ collisions [Too87], [Abe88] indicate that the mean rapidity shift $\langle\Delta y\rangle=\left\langle y_{\text {proj }}-y_{\text {leading }}>\right.$ increases as more nucleons are ejected from the target, evidence for multiple collisions. However, an adequate $p A$ data set still does not exist to trace out the energy dependence of nuclear stopping and the onset of nuclear transparency. Note that the SPS energy scale $\sqrt{s} \approx 20 \mathrm{GeV}$ is approximately the threshold for this crossover because for Lorentz $\gamma$ factors greater than

$$
\gamma=\frac{E_{c m}}{2 m}=\frac{\sqrt{s}}{2 m} \sim \frac{1}{2} \sqrt{s} \sim 10,
$$

Lorentz contraction saturates at the limiting nuclear thickness for typical nuclear distance scales of $2 R \sim 10 \mathrm{fm}$.

A natural consequence of this picture is the expectation of different physics in the fragmentation regions, where particles have longitudinal momentum close to the target and projectile, and the central region. The physics in the central region is determined by the soft, multiparticle production processes which dominate the high energy inelastic cross section [Gia79] and lead to peaks at midrapidity in the $d N / d y$ distribution for produced particles in $p p$ collisions [DeM82a]. The parton structure of the nucleons is important. The source of hadronic matter in the central region in $A A$ collisions is presumably a complicated superposition of nonperturbative QCD processes [Kaj83b]. Figure 1.2 has been drawn assuming that matter forms in 
an inside-outside cascade. The inside-outside cascade model, developed mainly by Bjorken in Ref. Bjo76, can account for limiting fragmentation [Ben69] and the weak energy dependence of central particle production (approximate Feynman scaling [Fey69]) observed in hadron-hadron interactions, and for transparency observed in hadron-nucleus collisions [Kaj82]. Its main feature: the $d N / d y$ distribution of produced particles will be a plateau which develops from the inside(slow particles with $y \sim 0$ created at times $t(y) \sim \tau_{0}$ within $\sim 1 \mathrm{fm}$ of the collision point) out (fast particles with $y \sim y_{\text {proj }}$ at later times and perhaps tens of fermis downstream). Matter forms at the edge of the fragmentation regions. Neglecting transverse motion, in one dimension of space plus one of time $(1+1)$, matter forms along the hyperbola

$$
\tau^{2}=t^{2}-z^{2},
$$

which leads to the famous light cone diagram of the inside-outside cascade, also shown in Figure 1.2. Because of the explicit correlation between rapidity and longitudinal position, Bjorken was also able to obtain his famous estimate for the energy density near midrapidity,

$$
\varepsilon_{0}=\frac{<m_{T}>\frac{d N}{\tau_{0} A_{T}}}{d y}
$$

where $A_{\Upsilon} \sim \pi R^{2}$ is the transverse area.

For the case of nuclear transparency, the participant nucleons are shifted toward midrapidity by $\Delta y \sim 0.7$ units, $^{1}$ the baryons in the fragmentation region are compressed, and high energy and particle densities are achieved in the baryonfree central region by multiparticle production. The mechanism for equilibration in both the central and fragmentation regions is the same as at lower energy, multiple collisions, but for times $\tau>\tau_{0}$. Because of the high particle densities, the mean free path in the central region may be very short and equilibration may take place very rapidly [Bjo83]. For this reason, calculations which employ the insideoutside cascade model typically assume that thermalization occurs immediately after materialization from the cascade [Kaj83a], [Kaj83b].

What about the subsequent expansion? The inside-outside cascade emphasizes the longitudinal development and neglects the transverse motion. It establishes the initial conditions for the expansion phase. Most hydrodynamic calculations hypothesize that near the collision axis, radially distant from the surface of the excited matter, the associated fluid velocity field is longitudinal [Bjo83], [Kaj83a], justifying a $1+1$ dimensional treatment, particularly at early times during the expansion. The following heuristic argument makes contact with the particle kinematic variables. It is well known that the mean transverse momentum $\left\langle p_{T}\right\rangle$ increases very slowly with $\sqrt{s}$ [Gia79] and depends only weakly on rapidity outside of the fragmentation regions, that is, the invariant cross section factorizes. The rapidity scale increases much faster, like $y \sim \ln \sqrt{s}$. So over most of the central region, the longitudinal velocities are much greater than the radial velocities.

\footnotetext{
${ }^{1}$ This is true because the leading baryon loses half of its energy on average in an $N N$ collision at high energy [Gia79].
} 
At what energy will a true central region develop, such that particle production occurs predominantly outside of the nuclear volumes and such that there is a clean separation of the central region from the fragmentation regions? The measured rapidity distributions for protons and negative hadrons permit us to address this question at $\sqrt{s}=20 \mathrm{GeV}$ in Chapter 6 .

\subsubsection{Thermodynamics}

Thermodynamics has played an important role in hadron physics ever since the pioneering early work of Fermi [Fer50] and Landau [Lan65]. The justification for this approach is more compelling for nucleus-nucleus collisions because of the large particle densities and large transverse dimensions. Thermodynamic models have been used widely both at relativistic [Das81] and ultrarelativistic [Cle86] energies. In this section we collect some results which will be employed in Chapter 6 to estimate the thermodynamic properties of the high density stage assuming thermal and chemical equilibrium.

For open systems, the first law of thermodynamics states

$$
d E=T d S-P d V+\sum_{i} \mu_{i} d N_{i}
$$

where $T$ is the temperature, $S$ is the entropy, $P$ is the pressure, $V$ is the volume, $N_{i}$ is the particle number of species $i$ and $\mu_{i}$ is its chemical potential. A system is open if the particle numbers are not fixed. Particle production, inelastic collisions and resonance decay are processes which change particle number during the evolution of a nucleus-nucleus collision. The chemical potential plays the same role for systems which exchange particles that the temperature plays for systems which exchange energy-two systems are in equilibrium with respect to particle transfer when $\mu_{1}=\mu_{2}$. It occupies a central position in discussions of phase ${ }^{2}$ and chemical equilibrium [Van66]. If all other thermodynamic quantities are held fixed, the chemical potential is the energy required to add one particle to an open system. For systems in which the log of the grand partition function $\ln Z$ is proportional to the volume, Equation 1.12 implies the following thermodynamic relation [Cle86] in terms of the energy density $\varepsilon$, the entropy density $s$ and the particle number densities $n_{i}$,

$$
\varepsilon+P=T s+\sum_{i} \mu_{i} n_{i}
$$

For the case of $\mu_{i}=0$, Equation 1.13 leads to simplifications in the hydrodynamic equations, in particular, a simple expression for the decrease in temperature during expansion. If $\mu_{i}=0$, Equations 1.12 and 1.13 imply

$$
c_{s}^{2}=\left(\frac{\partial P}{\partial \varepsilon}\right)_{V}=\frac{s}{T} \frac{\partial T}{\partial s} .
$$

\footnotetext{
${ }^{2}$ We refer to phase in the context of phase transitions, for example the quark gluon plasma phase compared to the hadronic phase. A hadronic resonance gas, constrained by baryon and strangeness conservation, may be in chemical equilibrium in the hadronic phase.
} 
The proper time dependence of the temperature follows from Equation 1.14 and Bjorken's scaling solution for the entropy density.

In thermal equilibrium at temperature $T$ and chemical potential $\mu$ the number of particles per unit volume of momentum space is given by the Fermi-Dirac or Bose-Einstein distribution function,

$$
\frac{d^{3} N}{d p^{3}} \propto \frac{1}{\exp [(E-\mu) / T] \pm 1}
$$

In Section 6.2 we discuss the different transverse momentum distributions which result from Equation 1.15 for different rapidity intervals. The temperature can be determined by fitting the $p_{T}$ distribution. In the Maxwell-Boltzmann limit the fits are particularly simple and the temperature is the only parameter, apart from overall normalization.

For the case of a phase transition between a quark gluon plasma and a hadronic gas, a relationship can be obtained [Cle86] between the baryon chemical potential $\mu_{\mathrm{b}}$ and the quark chemical potential $\mu_{\mathrm{q}}$,

$$
\mu_{\mathrm{b}}=3 \mu_{\mathrm{q}} \text {. }
$$

The criteria for equilibrium between different phases in an open system are that the temperature, pressure, and chemical potential be the same in every phase [Lan69], [Van66]. For processes that take place at constant temperature and pressure it is convenient to introduce the Gibbs free energy $G\left(T, P, N_{i}\right)=E-T S+P V$. Taking the differential and using Equation 1.12 leads to

$$
d G=-S d T+V d P+\sum_{i} \mu_{i} d N_{i}
$$

which implies the formal definition of the chemical potential

$$
\mu_{i}=\left(\frac{\partial G}{\partial N_{i}}\right)_{T, P} .
$$

Equation 1.16 results from minimizing the Gibbs free energy at constant temperature and pressure and requiring baryon number conservation,

$$
N_{\mathrm{q}} / 3+N_{\mathrm{N}}=\text { constant, }
$$

where $N_{\mathrm{N}}$ and $N_{\mathrm{q}}$ are the excess of nucleons over antinucleons and quarks over antiquarks, respectively.

In statistical mechanics the energy and particle number of many particle, open systems are defined as average values over the grand canonical ensemble and can be derived from the grand partition function [Fin69]. In particular,

$$
n=\frac{\langle N\rangle}{V}=\frac{T}{V} \frac{\partial}{\partial \mu} \ln Z \text {. }
$$


Equation 1.20 for the net quark number density $n_{\mathrm{q}}=N_{\mathrm{q}} / V$ can be evaluated for free particles both for the case of massless fermions and for the case of massless bosons at zero chemical potential [Mul85], [Cle86]. The result for the net baryon number density $n_{\mathrm{b}}$ of a two flavor quark gluon plasma is

$$
n_{b}=\frac{1}{3} n_{q}=\frac{2}{3}\left(\mu_{\mathrm{q}}^{3}+\frac{\mu_{\mathrm{q}} T^{2}}{\pi^{2}}\right) .
$$

For the case of scaling hydrodynamics, $n_{\mathrm{b}}$ in the high density stage can be estimated from the the measured baryon rapidity distribution. Under the assumptions that Equation 1.21 has been valid early in the collision and that the chemical potentials are small we can estimate the quark, and hence the baryon, chemical potential if we can deduce temperatures at earlier times from $T_{f}$, the measured temperature at freezeout. We will make this estimate in Chapter 6 using the results of this and the next section.

\subsubsection{Hydrodynamic Expansion}

Hydrodynamics has been used extensively to calculate the space-time evolation of nucleus-nucleus collisions [Cla86]. The two fundamental assumptions under which the hydrodynamic equations are valid, that the number of degrees of freedom is large and that the mean free path is short, are reasonably well satisfied during the compression, high density, and early expansion stages of the collision. As we mentioned in Section 1.2.1, under the influence of the seminal papers by Landau [Lan65] and Bjorken [Bjo83], at high energy most theoretical efforts have emphasized the longitudinal motion. Discussions of the complicated transverse motion, when the expansion becomes $(1+3)$ dimensional, can be found in [Bay83] and [Cle86]. We neglect it. We trace out the derivation of the scaling solution to establish a connection between the observable rapidity density $d N / d y$ and the particle density per cubic fermi, ${ }^{3}$ and to obtain a simple expression for the time dependence of the temperature during expansion.

The hydrodynamic equations of motion express energy and momentum conservation in terms of the stress tensor $T^{\mu \nu}$,

$$
\partial_{\mu} T^{\mu \nu}=0,
$$

where for a perfect fluid

$$
T^{\mu \nu}=(\varepsilon+P) u^{\mu} u^{\nu}+P g^{\mu \nu} .
$$

In Equation 1.23, $g^{\mu \nu}$ is the metric tensor and $u^{\mu}$ is the four-velocity of a fluid element with local velocity $\vec{v}$

$$
u^{\mu}=\gamma(1, \vec{v}) \text {. }
$$

\footnotetext{
${ }^{3}$ Although we emphasize the baryon density $n_{b}$ because we will estimate the baryon chemical potential from our measurement of the proton rapidity distribution, ideal gas thermodynamics at $\mu=0$ shows that $s \propto n$ so that this connection also applies to produced particles [Gyu84].
} 
By contracting Equation 1.23 with $u_{\mu}$ and employing Equations 1.12 and 1.13 it can be shown that baryon number conservation, expressed in the form of a fluid continuity equation

$$
\partial_{\mu}\left(u^{\mu} n_{b}\right)=0,
$$

implies entropy conservation,

$$
\partial_{\mu}\left(u^{\mu} s\right)=0 \text {. }
$$

In $(1+1)$ dimensional hydrodynamics, after parametrizing the four velocity by

$$
u^{\mu}=(\cosh \theta, \sinh \theta, 0,0),
$$

and changing variables to the space-time rapidity and the proper time

$$
y=\frac{1}{2} \ln \frac{t+z}{t-z}, \quad \tau=\sqrt{t^{2}+z^{2}},
$$

the conservation equation for $n_{\mathrm{b}}$ becomes

$$
\tau \frac{\partial n_{\mathrm{b}}}{\partial \tau}+n_{\mathrm{b}} \frac{\partial \theta}{\partial y}+\tanh (\theta-y)\left(\frac{\partial n_{\mathrm{b}}}{\partial y}+n_{\mathrm{b}} \tau \frac{\partial \theta}{\partial \tau}\right)=0
$$

with an identical equation for the entropy density. We will see below that for Bjorken's scaling solution, the fluid space-time rapidity can be identified with the particle longitudinal rapidity of Equation 1.3. This identification enables one to estimate the baryon density at early times from the measured proton rapidity distribution. The transformation equations back to ihe time and space representation are

$$
t=\tau \cosh y, \quad z=\tau \sinh y .
$$

Bjorken's scaling hypothesis, motivated by the observation of a plateau in the rapidity distribution of produced particles [Gia79], asserts that for nucleus-nucleus collisions the initial conditions in the central region are invariant under Lorentz transformations. In the inside-outside cascade model the scaling hypothesis is embodied by the specification of initial conditions, which are independent of rapidity, on a surface of constant proper time at $\tau=\tau_{0}$. Bjorken argued that the symmetry with respect to Lorentz transformations of the initial conditions must be preserved by the dynamics which implies that the hydrodynamic equations must be covariant under Lorentz boosts, i.e, under small shifts in rapidity. From Equation 1.29 it is clear that this will be true if

$$
\theta(\tau, y)=y,
$$

which leads to a dramatic simplification in the hydrodynamic equations, in particular,

The solutions are

$$
\frac{d n_{\mathrm{b}}}{d \tau}=-\frac{n_{\mathrm{b}}}{\tau}, \quad \frac{d s}{d \tau}=-\frac{s}{\tau} .
$$

$$
n_{\mathrm{b}}(\tau)=n_{\mathrm{b}}\left(\tau_{0}\right) \frac{\tau_{0}}{\tau}, \quad s(\tau)=s\left(\tau_{0}\right) \frac{\tau_{0}}{\tau} .
$$


Note that Equation 1.31, together with the definition of the space component of the fluid four-velocity $u^{1}=\gamma v_{z}$, implies that

$$
v_{z}=\frac{z}{t}
$$

throughout the expansion, not only as an initial condition. This establishes the correspondence with the particle rapidity [McL86]

$$
y=\frac{1}{2} \ln \frac{1+p / E}{1-p / E}=\frac{1}{2} \ln \frac{1+v_{z}}{1-v_{z}}=\frac{1}{2} \ln \frac{t+z}{t-z} .
$$

Note the difference between the initial conditions in the Bjorken picture and in Landau's model, where at $t=0$ the fluid is assumed to be at rest, completely stopped by the collision. Because of this difference, Landau derived his asymptotic solution for the charged particle $d N / d y$ distribution ${ }^{4}$

$$
\frac{d N}{d y} \propto \frac{1}{\sqrt{2 \pi \sigma^{2}}} \exp \left(-y^{2} / 2 \sigma^{2}\right), \quad \sigma^{2}=\frac{1}{2} \ln \left(s / 4 m_{p}^{2}\right),
$$

whereas Bjorken obtained his scaling solution from the assumption of a central plateau in the rapidity density. We will use both the Bjorken and Landau pictures to interpret the negative hadron and proton $d N / d y$ distributions in Chapter 6.

An important consequence of the scaling solution is

$$
\begin{aligned}
\frac{d N_{\mathrm{b}}}{d y}=\frac{1}{d y} \int n_{\mathrm{b}} d^{3} x=\frac{1}{d y} \int n_{\mathrm{b}}(\tau) \tau d y d^{2} x_{T} & =\int n_{\mathrm{b}}\left(\tau_{0}\right) \tau_{0} d^{2} x_{T} \\
& =n_{\mathrm{b}}\left(\tau_{0}\right) \tau_{0} A_{T},
\end{aligned}
$$

where we have used the volume element in a frame in which the fluid is at rest,

$$
d^{3} x=\tau d y d^{2} x_{r}
$$

The baryon density at early times can be determined from a measurement of the baryon density per unit of rapidity at freezeout. Furthermore,

$$
\frac{d}{d \tau}\left(\frac{d N_{\mathrm{b}}}{d y}\right)=\frac{d}{d \tau}\left(n_{\mathrm{b}} \tau\right) \int d^{2} x_{T}=0,
$$

the baryon content per unit of rapidity is a constant of the motion, independent of the equation of state. From Equation 1.14, if the baryon density or, equivalently, the baryon chemical potential, is small the temperature evolution during the expansion is given by

$$
T(\tau)=T\left(\tau_{0}\right)\left(\frac{\tau_{0}}{\tau}\right)^{c_{0}^{2}}
$$

If we can estimate the duration of the expansion we can determine the temperature at $\tau_{0}$ from $T_{f}$, the temperature at freezeout.

\footnotetext{
${ }^{4}$ The expression appearing in Landau's original paper is for the pseudorapidity density $d N / d \eta$ where $\eta=\frac{1}{2} \ln \frac{1+\cos \ell}{1-\cos \theta}$. The substitution $\eta \rightarrow y$ is justified in Ref. Car72.
} 


\section{Chapter 2}

\section{Experiment NA35 at the CERN SPS}

Experiment NA35 at the CERN SPS was originally conceived as a somewhat lower energy Proton Synchrotron(PS) experiment. A proposal was made before the PS Program Advisory Committee for a combined Streamer Chamber-Plastic Ball experiment [PSC82]. This experiment was approved at the available energies of 9-13 GeV/nucleon.

A number of technical problems and physics arguments conspired to transform this proposal into an SPS experiment. The difficulty of moving a large superconducting magnet from CERN's North Area inspired the suggestion [Har89] of using the SPS ring as a passive beam transport. Once the idea of heavy ions circulating in the SPS had been accepted it was a logical step to accelerate them. The increased rapidity gap between the fragmentation regions and midrapidity would enable a cleaner separation of the fireball from spectator matter. The greater than factor of 4 increase in $\sqrt{s}$ might lead to a large increase in the energy density [McL82]. NA35 would have the additional advantage of the availability of $p p$ data at $200 \mathrm{GeV} / \mathrm{c}$ from the same detector [DeM82a] in order to investigate departures from superposition in $p A$ and $A A$ physics. The move to the SPS resulted in a huge change in scale of the heavy ion program at CERN with six large collaborations eventually participating in major experiments [PQM88].

There have been two production running periods as of June 1990. In the fall of $1986{ }^{16} \mathrm{O}$ beams were accelerated to 60 and $200 \mathrm{GeV} /$ nucleon as well as proton beams at the same energies. In the fall of $1987^{32} \mathrm{~S}$ beams were accelerated to 200 $\mathrm{GeV} /$ nucleon. Data were taken for a variety of nuclear targets ranging in mass from $\mathrm{Al}$ to $\mathrm{Pb}$.

\subsection{Ions In the SPS}

The complex of accelerators employed for the acceleration of light ions to 60 and $200 \mathrm{GeV} /$ nucleon is shown in Figure 2.1. An electron cyclotron resonance (ECR) ion source, developed at Grenoble [Gel82] and built in collaboration with GSI- 


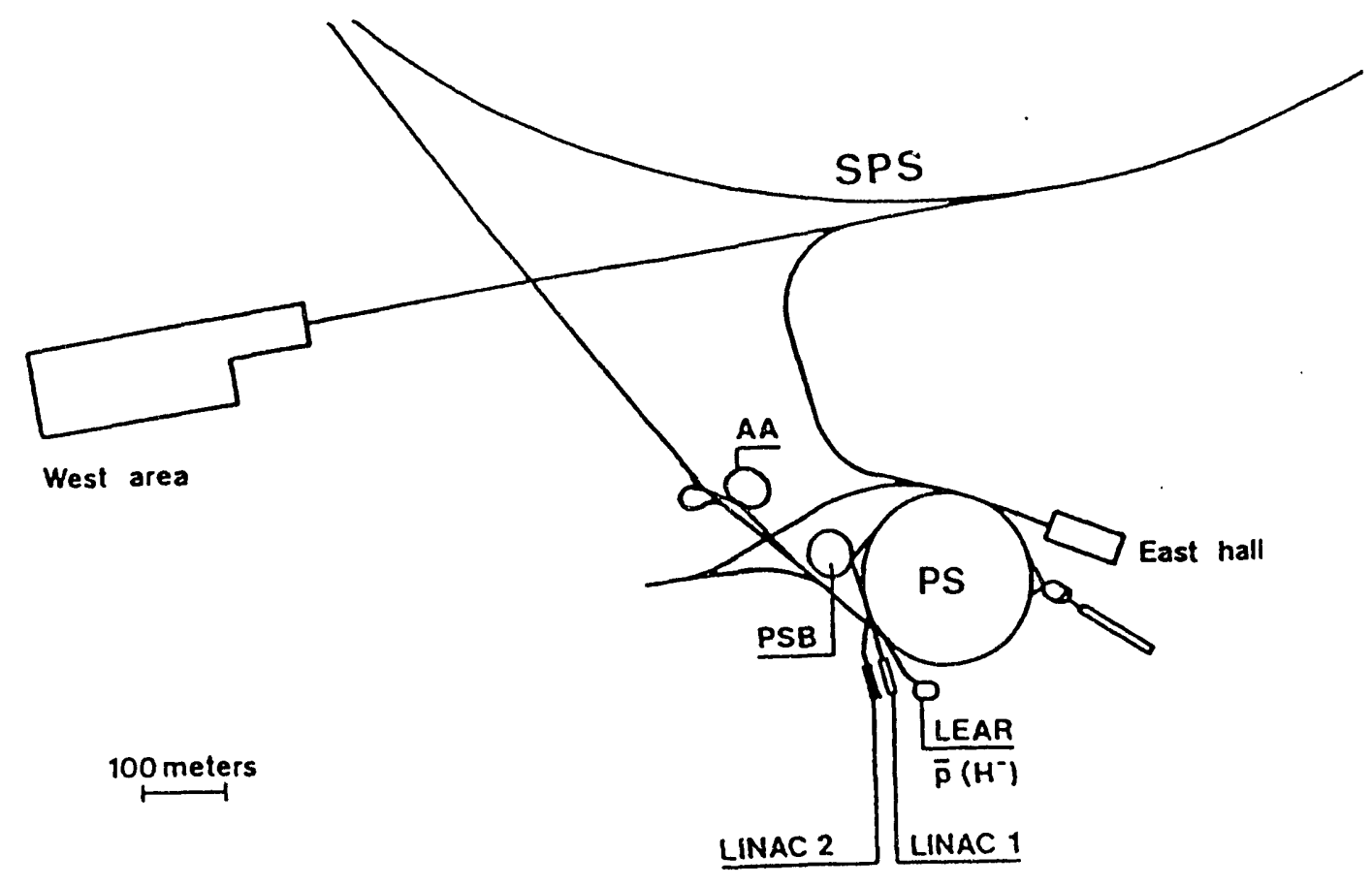

Figure 2.1: CERN complex employed for the acceleration of light ions. Ions from Linac 1 are injected into the PS, accelerated to $10 \mathrm{GeV} /$ nucleon, then accelerated to full energy in the SPS ring and delivered to the experimental areas.

Darmstadt, and a radio frequency quadrupole (RFQ) cavity preaccelerator built at LBL delivered up to $100 \mu \mathrm{A}$ of high charge state $\left(\mathrm{O}^{6+} / \mathrm{S}^{12+}\right)$ ions into the PS's Linac 1 [Wol86]. After acceleration to a kinetic energy of $12 \mathrm{MeV} /$ nucleon and complete stripping, a $10 \mu \mathrm{A}$ beam was injected into the PS booster and subsequently into the PS at $260 \mathrm{MeV} /$ nucleon. A $10 \mathrm{GeV} /$ nucleon beam was extracted from the PS and injected into the SPS for final acceleration. Beams of approximately $10^{7}$ ions/s intensity were extracted from the SPS and fed into the external beam transport system for delivery to the experimental halls [Dar88]. NA35 is located in the North experimental area. It shared the $\mathrm{H} 2$ beam line with experiment NA36. The overall spill cycle at $200 \mathrm{GeV} /$ nucleon was $14.4 \mathrm{~s}$ with a $4.4 \mathrm{~s}$ extraction. At $60 \mathrm{GeV} /$ nucleon the spill length was longer, $\simeq 6 \mathrm{~s}$.

\subsection{NA35 Experimental Overview}

Experiment NA35 is a combined streamer chamber, calorimeter experiment designed as a survey study of nucleus-nucleus collisions at 60 and $200 \mathrm{GeV} /$ nucleon. The detector layout is shown in Figure 2.2. ${ }^{16} \mathrm{O}$ and ${ }^{32} \mathrm{~S}$ ion beams, collimated down to an intensity of about $10^{5}$ ions/s, passed through a set of beam-defining counters. The projectile nuclei strike nuclear targets positioned $\sim 8 \mathrm{~cm}$ upstream 


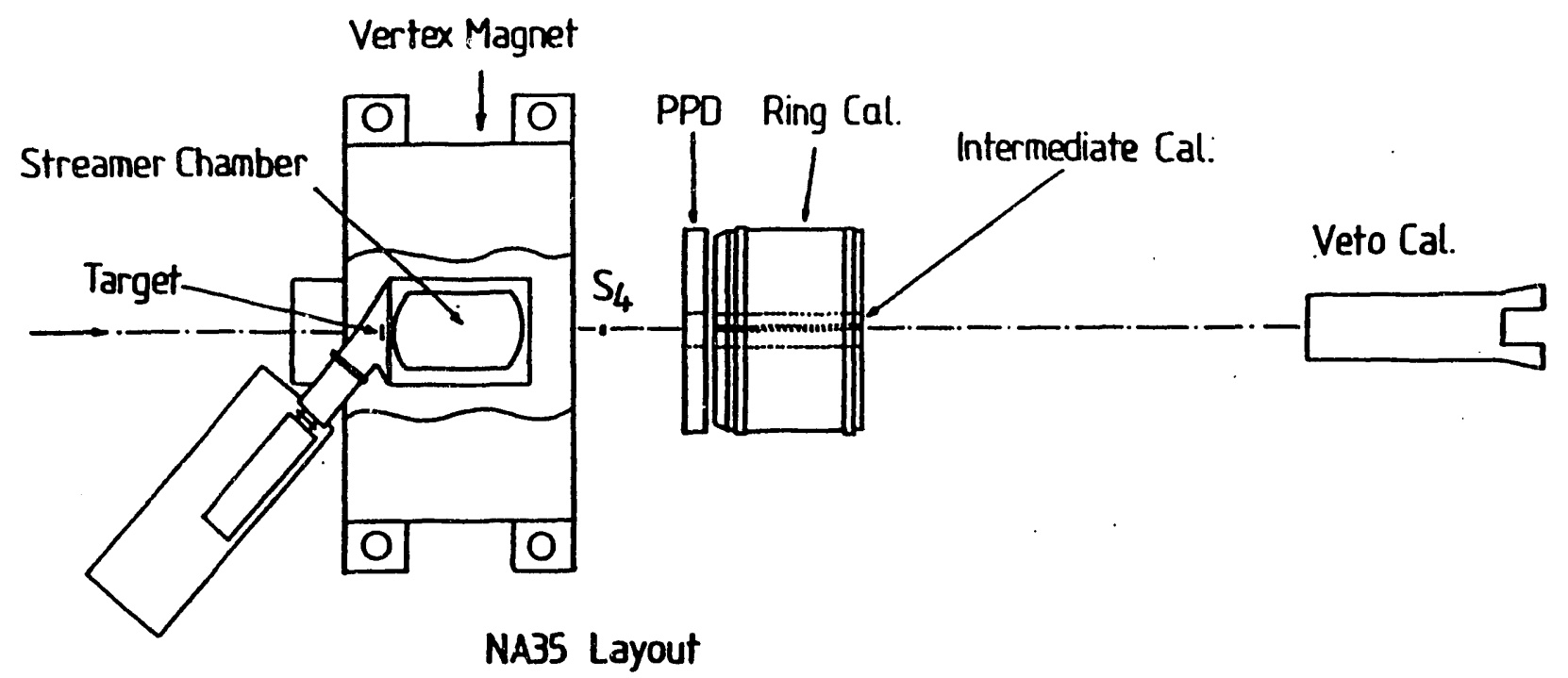

Figure 2.2: NA35 experimental apparatus. The beam enters from the left. The Photon Position Detector (PPD), Veto calorimeter and scintillator $\mathrm{S}_{4}$ provide different event triggers.

of the streamer chamber entrance window. ${ }^{1}$ The streamer chamber, constructed inside a large superconducting magnet, was previously employed in experiments NA5 [DeM82a] and NA9 [Alb83]. It can measure exclusive, final state charged particle multiplicities, inclusive charged particle spectra, charged particle correlations, and strange particle spectra and yields from $V^{0}$ decays and from the charged $K$ decays $K^{ \pm} \rightarrow \pi^{ \pm} \pi^{0}, K^{ \pm} \rightarrow \pi^{ \pm} \pi^{ \pm} \pi^{\mp}$ in its fiducial volume. Two meters downstream from the streamer chamber is a scintillator, denoted $S_{4}$ in Figure 2.2, which provided a minimum bias trigger. The calorimeter complex is located about seven meters downstream from the streamer chamber. These calorimeters were used previously in experiments NA5 and NA24 [DeM87a], [DeM87b]. They can be used as triggering devices for the streamer chamber and can measure forward energy flow $E_{V \text { eto }}$ into a veto calorimeter, transverse energy $E_{T}$ in a set of mid and intermediate rapidity devices, $E_{V \text { eto }}-E_{T}$ correlations, and subdivide the calorimetric signal into electromagnetic and hadronic components. A small hole in the forward calorimeter allowed passage of non-interacting beams on to NA36.

\footnotetext{
${ }^{1}$ Virtually all of the streamer chamber data which has been analyzed were taken with external targets located outside of the chamber. Some internal target runs were taken and deemed unmeasurable, mainly because of obscuration by low-momentum, spiralling electrons.
} 


\subsubsection{Streamer Chamber}

A streamer chamber is a visual tracking detector with good two-track resolution, excellent multi-track efficiency, and large solid angle acceptance. The trajectories of charged particles are imaged in several camera views and measured. Charged particle momenta can then be determined by stereoscopic reconstruction in a magnetic field.

The primary ionization electrons left along the path of a charged particle as it traverses the chamber gas are accelerated by an applied electric field. When a primary electron reaches sufficiently high energy it can cause secondary ionizations which successively multiply to create an avalanche, a teardrop-shaped space charge structure of electron-ion pairs. When the local electric field generated by the avalanche space charge distribution reaches a significant fraction of the applied field, ultraviolet photons emitted by excited atoms produce secondary avalanches [Kle86] and the avalanche is transformed into a streamer. The electric field is terminated before the streamer can grow into a complete spark discharge. By varying the strength and the duration of the electric field chambers can be operated in either avalanche mode or streamer mode. The luminosity of both avalanches and streamers is a consequence of radiative recombination with a color characteristic of the chamber gas. A complete discussion of the process of streamer and avalanche formation can be found in Ref. Ric74 and Ref. Bul67. Streamers are relatively bright and can be imaged with conventional photography onto sensitive films or seen by the human eye. In order to detect avalanches, image intensifiers must be employed. Running in or near the avalanche mode has several advantagesintrinsically superior position resolution, reduced production of flares, which are spark discharges between the high voltage(HV) electrodes which provide the electric field, and preservation of the primary ionization distribution which facilitates particle identification [Bas81].

The NA35 streamer chamber [Alb83] is a large chamber with a sensitive volume of $2.0 \times 1.2 \times .72 \mathrm{~m}^{3}$. It rests in a superconducting vertex magnet with a central magnetic field strength $\vec{B}$ of 1.5 Tesla. Because of the size of the chamber the field varies by as much as $20 \%$ near the edges of the fiducial volume. The electric field $\vec{E}$ is established between grid electrodes inside the chamber. A schematic diagram of the chamber displaying the grid configuration and the orientation of the $\vec{E}$ and $\vec{B}$ fields is shown in Figure 2.3. In order to avoid a central electrode near the beam the chamber is divided into three gaps by two electrodes. HV pulses of opposite polarities are produced in a $375 \mathrm{kV}$ Marx generator with two parallel discharge tubes, one at each polarity. The HV pulses are shaped to a width of 12 nsec (FWHM) by a double Blumlein transmission line with a single spark gap. The discharge takes place between the two polarities to ensure that both pulses arrive at the chamber simultaneously [GSI85]. The chamber performance was extremely stable with very small pulse-to-pulse variations of the peak $\vec{E}$ field and good field homogeneity along the length of the chamber.

The gas volume is enclosed by a transparent, mylar foil $120 \mu \mathrm{m}$ thick. Visible, charged particle trajectories were imaged through the mylar on the top face 


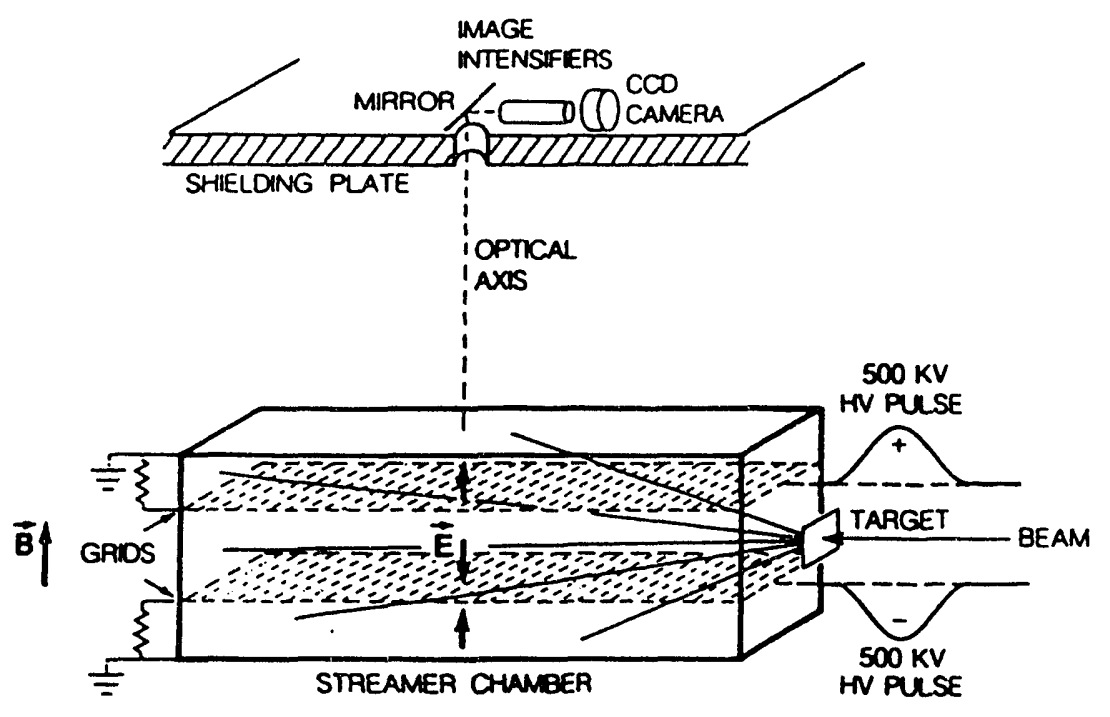

Figure 2.3: NA35 streamer chamber. The electric $\vec{E}$ and magnetic $\vec{B}$ fields are parallel. The grid electrodes act as wave guides for the HV pulse. Terminating the chamber at its wave impedance through termination resistors ensures homogeneity of $\vec{E}$ along its length. Film and CCD cameras are housed in a camera pavilion above the chamber.

of the chamber. The front support face is polyethylene except for a thin window consisting only of the mylar foil. Because of the strong, kinematic forward focusing of energetic particles in the laboratory rest frame, virtually all particles enter the chamber through the thin window, reducing the probability of secondary interaction.

The chamber is filled with a Neon-Helium gas mixture $(80 \%-20 \%)$ at atmospheric pressure. The memory time of the chamber was reduced to $10 \mu \mathrm{s}$ by the addition of $0.05 \mathrm{ppm}$ of the electronegative gas $\mathrm{SF}_{6}$. The addition of $0.25 \%$ isobutane allowed for a $10 \%$ reduction in operating voltage while keeping the streamer light output constant. Because of the anticipated high track density it was essential to optimize the spatial resolution. Operating the chamber near the avalanche mode resulted in a streamer length of several $\mathrm{mm}$ and a streamer diameter of about $1 \mathrm{~mm}$. A complete technical description of the NA35 streamer chamber can be found in Ref. Wen90.

Streamer chamber pictures were recorded by two independent imaging systems, a film camera system that was previously used by UA5 [Aln87] and a chargecoupled device (CCD) camera system, developed specifically for NA35 [Tin87], that produces direct digital images. Both devices utilized image intensifiers to record the faint avalanche/streamers. A more complete discussion of the film camera imaging system can be found in Ref. San87. The data analyzed in this thesis were taken with the CCD camera system. It also served as an online monitor, providing event-by-event visual feedback on chamber and trigger performance and a quantitative estimate of the centrality of the trigger from an online measurement 
of the charged particle multiplicity. The design, construction and implementation of the CCD system, and the development of novel data reduction techniques whose objective was the automation of visual detector event processing, comprise a large portion of this thesis.

A film image of a $200 \mathrm{GeV} /$ nucleon ${ }^{32} \mathrm{~S}+{ }^{32} \mathrm{~S}$ collision is shown in Figure 2.4. With field on, because of the external target, the streamer chamber has a gradual onset of its acceptance, reaching full acceptance for pions at $y \simeq 0.4$ and for protons at $y \simeq 0.2$. The number of charged particles detected in Figure 2.4 is $\sim 300$. The bright, typically circular, spots are flares caused by charged particles passing close to the grid electrodes or energetic $\delta$-electrons. The extremely bright section at the center of the picture and running nearly the full length of the image is the central cone or beam pencil. It is formed by the superposition of many tracks. The forward cone corresponds to laboratory angles $\theta_{\text {lab }}<6^{\circ}$. The projected track density in the cone exceeds the streamer chamber plus optical resolution and leads to a rapid reduction in detection efficiency and sharp cutoffs in the acceptance for rapidities greater than $y \sim 3.0$. The streamer chamber acceptance for CCD-imaged data will be discussed in detail in Chapter 5 .

The relatively faint, illuminated crosses which appear within the image and the much brighter ones outside of the chamber sensitive volume are fiducial marks, reference marks at precisely known positions which are required for the reconstruction of space curves from projected film or CCD images. The chamber was equipped with nine fiducial marks on the back plane and ten fiducial marks outside the front window. In order to correct for distortions caused by the image intensifiers and the imperfect lenses a calibration grid of stretched fluorescent wires was placed in front of the chamber and recorded at periodic intervals. From the surveyed positions and images of the fiducials and grids a self-consistent set of optical constants and a procedure for distortion correction were determined.

With a memory time of $10 \mu$ s the chamber can be operated at a beam intensity of $10^{5}$ ions/s. The HV power supplies can recharge the Marx generator at a rate of 3-4 Hz. However, the $\sim .6 \mathrm{~s}$ decay time of images on the output phosphors of the image intensifiers sets an upper bound of 1.5 frames/s with the actual data rate depending on film transport or, for the case of the CCD's, the readout rate of the large digital images. The film data acquisition recorded 3 events/spill, the CCD system recorded 1 event/spill. The CCD system data acquisition is discussed in Chapter 3.

\subsubsection{Calorimetry}

There were four calorimeters employed in NA35. A photon position detector (PPD) [Art84] and a ring calorimeter (Ring) [DeM83a] measured mid-rapidity particles. A downstream veto calorimeter (Veto) [DeM83a] measured the projectile fragmentation region. Both calorimeters have been used in previous experiments. An intermediate calorimeter served as an active collimator [Mar91] in order to restrict the acceptance of the Veto to the projectile fragmentation region.

The PPD is a highly segmented, gas sampling shower detector with coordinate 


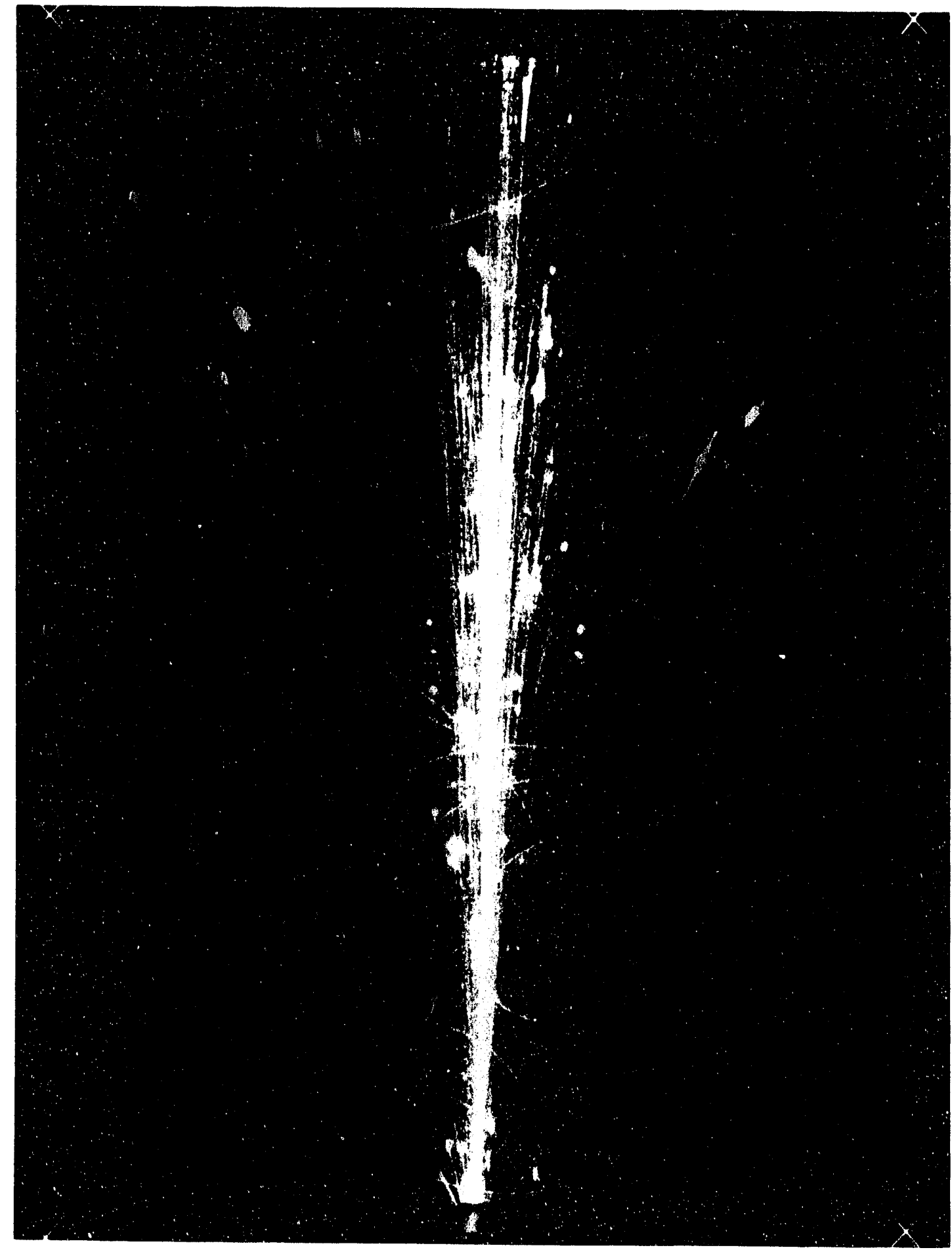

Figure 2.4: Film image of $\mathrm{a}^{32} \mathrm{~S}+{ }^{32} \mathrm{~S}$ collision at $200 \mathrm{GeV} /$ nucleon incident energy. 
readout. In experiment NA24 [DeM87a], [DeM87b] it was used to separate direct photons from the large background from the decay $\pi^{0} \rightarrow \gamma \gamma$. For low multiplicities the high granularity of the PPD leads to nearly $100 \%$ efficiency for $\pi^{0}$ mass reconstruction if the $\gamma-\gamma$ separation is greater than $3 \mathrm{~cm}$. For the high multiplicities encountered in NA35 no attempt was made to reconstruct individual showers in any of the calorimeters. In NA35 the PPD was used to measure the electromagnetic component of the transverse energy $E_{T}$. Its sensitive area is $3.0 \times 3.0 \mathrm{~m}^{2}$ excluding a $0.5 \times 0.5 \mathrm{~m}^{2}$ square central hole. Electromagnetic showers are sampled by 12 sensitive layers of triangular proportional tubes sandwiched with 8 layers of lead converter totalling 9.6 radiation lengths $X_{0}$ and 0.5 nuclear absorption lengths $\lambda_{\text {int }}$. A subset of its 6144 horizontal and vertical sense wires are summed directly in hardware so it can serve as a trigger device on a $\sim 200 \mathrm{~ns}$ time scale, more than an order of magnitude shorter than the memory time of the streamer chamber. The PPD is too short to fully contain high energy showers so the combined PPD/Ring system was calibrated with electrons from 5 to $170 \mathrm{GeV}$ and with hadrons from 5 to $60 \mathrm{GeV}$. The energy resolution for incident electrons was determined to be $\sigma(E) / E=0.30 / \sqrt{E}$. The PPD is thick enough to contain almost all the electromagnetic energy incident upon it at midrapidity [Hec88]. The angular acceptance of the PPD in pseudorapidity $\eta$ at $200 \mathrm{GeV} /$ nucleon is $2.2<\eta<3.6$ with full $2 \pi$ coverage in azimuth $\phi$.

The Ring is an intermediate granularity, sampling shower detector with electromagnetic and hadronic sections. It was used in experiment NA5 to search for jets with a large acceptance calorimeter trigger [DeM82b], [DeM83b]. In NA24 it completed the energy measurement for electromagnetic showers which leaked out the PPD. In NA35 the Ring was used to measure the hadronic component of $E_{T}$. Its sensitive area is a circle of $3.0 \mathrm{~m}$ diameter excluding a $56 \mathrm{~cm}$ diameter central hole around the beam axis. The electromagnetic section is comprised of 16 layers of lead-scintillator sandwich totalling $16.0 X_{0}$. The hadronic section consists of 20 layers of iron-scintillator sandwich. Together, the electromagnetic and hadronic sections total $8.0 \lambda_{\text {int }}$, enough to fully contain $200 \mathrm{GeV}$ hadronic showers. The Ring is divided into 240 cells, 24 in azimuth $\phi$ and 10 in polar angle $\theta$. A nonuniform segmentation in $\theta$ was employed to obtain an angular acceptance of \pm one unit of $\eta$ per cell about midrapidity at $200 \mathrm{GeV} /$ nucleon. A novel light collection system [Eck78], utilizing different wavelength shifter material in the electromagnetic and hadronic sections, enabled both sections to be read out optically in a common acrylic rod. The Ring was calibrated with a test module from $2-10 \mathrm{GeV}$ in the PS and directly from $20-60 \mathrm{GeV}$ in the SPS. For the electromagnetic section the energy resolution determined from incident electrons was $\sigma(E) / E=.006+0.14 / \sqrt{E}$. For the hadronic section the energy resolution determined from incident pions was $\sigma(E) / E=.003+0.64 / \sqrt{E}$. The angular acceptance of the Ring at $200 \mathrm{GeV} /$ nucleon is $2.3<\eta<3.9$ with complete $\phi$ coverage. The Ring is mounted on movable rails so that the longitudinal distance from the target can be varied in order to select approximately the same acceptance at different incident beam energies.

The Veto is a coarse granularity, sampling shower detector with electromagnetic 


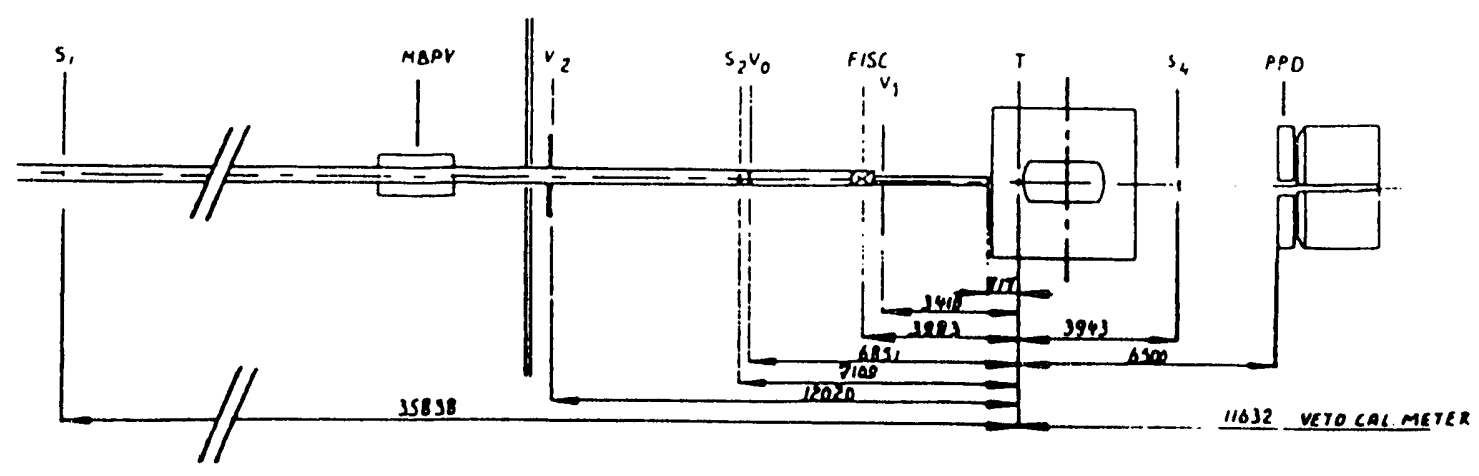

Figure 2.5: NA35 beam counters. Also shown are the trigger scintillator $\mathrm{S}_{4}$ and the PPD which provide the minimum bias and $E_{T}$ triggers, respectively.

and hadronic sections. It was used in NA24 to measure energy flow at small angles and help reject background [DeM87a]. In NA35 it was used to measure the forward energy $E_{V \text { eto }}$ in the projectile fragmentation region. Its sensitive area has an octagonal shape and covers approximately $1.0 \times 1.0 \mathrm{~m}^{2}$ with a small central hole of $2.5 \mathrm{~cm}$ diameter which allowed non-interacting beams to pass through to experiment NA36. For certain running periods this hole was plugged to obtain a better measurement. In fact, with the Veto transmitting, the detector was not an effective trigger device nor could it make a consistent energy measurement [Mar91]. The electromagnetic section contains 16 layers of $5.5 \mathrm{~cm}$ thick lead sandwiched with $2.5 \mathrm{~cm}$ scintillator totalling $16.0 X_{0}$. The hadronic section contains 25 layers of 5.0 $\mathrm{cm}$ thick iron sandwiched with $2.5 \mathrm{~cm}$ scintillator. The detector totals $9.6 \lambda_{\text {int }}$. It is subdivided into four cells with separate electromagnetic and hadronic readout. The Veto was calibrated with incident electrons from 15 to $100 \mathrm{GeV}$ and pions from 15 to $340 \mathrm{GeV}$. The energy resolution for incident electrons, which are contained in the electromagnetic section, is $\sigma(E) / E=0.17 / \sqrt{E}$. The energy resolution for pions is $\sigma(E) / E=0.03+0.74 / \sqrt{E}$.

\subsection{Trigger and Event Selection}

${ }^{16} \mathrm{O}$ and ${ }^{32} \mathrm{~S}$ beams were defined by a series of counters, depicted schematically in Figure 2.5. Ion beams are selected by an upstream, $5 \mathrm{~mm}$ thick, Cerenkov-emitting quartz counter $S_{1}$ in coincidence with a $0.3 \mathrm{~mm}$ thick scintillation counter $S_{2}$ closer to the target. The Cerenkov counter had single charge resolution up to $Z=16$ so it could select ${ }^{32} \mathrm{~S}$ ions of the proper charge state and reject contaminations in the beam from ions with the same charge-to-mass ratio. The veto counters $V_{0}, V_{1}$ and $V_{2}$ reject events with beam halo particles and events which result from upstream interactions in $S_{1}$ and $S_{2}$.

Three types of triggers were employed by NA35:

1. A minimum bias or interaction trigger was derived from a $1.0 \mathrm{~cm}$ diameter, 10 
mm thick scintillation counter, denoted $S_{4}$ in Figure 2.5, located $4 \mathrm{~m}$ downstream from the target. $S_{4}$ subtended the projectile fragmentation region. Minimum bias events were obtained by selecting interactions which produced a pulse height below the projectile peak. A class of soft nuclear and electromagnetic interactions cannot be separated from the projectile peak [Bam88] and contribute a trigger bias for peripheral events. Minimum bias data were used to measure the total inelastic cross sections $\sigma_{\text {tot }}$ which exhibited the target and projectile mass dependence established at low-energy [Bam88],

$$
\sigma_{\text {tot }}=\pi R_{0}^{2}\left[A_{p}^{1 / 3}+A_{t}^{1 / 3}-\beta\left(A_{p}^{-1 / 3}+A_{t}^{-1 / 3}\right)\right]^{2} .
$$

The data were well fit with the values determined at $2.1 \mathrm{GeV} /$ nucleon [Hec78], $R_{0}=1.36 \mathrm{fm}$ and $\beta=1.11$. Minimum bias data were also used to study the correlations between $E_{T}, E_{V \text { eto }}$, and the charged particle multiplicity $n_{c h}$. For ${ }^{32} \mathrm{~S}+{ }^{32} \mathrm{~S}$ at $200 \mathrm{GeV} /$ nucleon, $\sigma_{\text {tot }}=1.8$ barns and $\left\langle n_{\text {ch }}\right\rangle=75$.

2. A forward energy trigger was derived from the Veto by selecting events with leading energy $E_{V \text { eto }}$ less than a certain threshold. The vertex magnet has an analyzing power of $4.32 \mathrm{~T}$-m which adds a $1 \mathrm{GeV} / c p_{T}$ kick in the bend plane to high momentum charged particles [Hec88]. The Veto acceptance was extended asymmetrically to detect deflected projectile fragmentation protons. The most central sample which has been analyzed for ${ }^{32} \mathrm{~S}+{ }^{32} \mathrm{~S}$ was a film data set for a "hard Veto" trigger which corresponded to a trigger cross section $\sigma_{\text {trig }}=34$ mbarns, $2 \%$ of the total inelastic cross section. For this event class $\left\langle n_{c h}\right\rangle=229$. These events were selected by requiring a forward energy $E_{V \text { eto }}<1200 \mathrm{GeV}$. The differential cross section $d \sigma / d E_{V \text { eto }}$ fell precipitously for $E_{V \text { eto }}<1000 \mathrm{GeV}$ [Mar91], suggesting that there were $\sim 5$ spectator nucleons even in the most central collisions of light, equal-mass nuclei. This interpretation was confirmed by Monte Carlo studies. The Veto trigger was relatively unbiased with respect to transverse observables. For ${ }^{16} \mathrm{O}+\mathrm{Au}$ at $200 \mathrm{GeV} /$ nucleon, the differential cross section $d \sigma / d E_{T}$ for hard Veto events was Gaussian distributed [Hec88].

3. A transverse energy $E_{T}$ trigger was derived from the PPD by selecting events which deposited energy greater than a certain threshold. Because of the PPD acceptance, $E_{T}$ trigger events were generated mainly by produced particles. The hadronic energy measured by the PPD is redistributed by the magnetic field. Two-thirds of the energy captured by the PPD is neutral in origin-as a trigger device it was found to be insensitive to the magnetic field [Hec88]. The ${ }^{32} \mathrm{~S}+{ }^{32} \mathrm{~S}$ data analyzed in this thesis were taken with a "medium $E_{T}$ " trigger which corresponded to $\sigma_{\text {trig }}=200$ mbarns, $11 \%$ of $\sigma_{\text {tot }}$. This event class was selected by requiring $E_{T}>25 \mathrm{GeV}$ in the PPD. Since approximately $40 \%$ of the transverse energy is electromagnetic [Mar91], this corresponds to $E_{T} \gtrsim 60 \mathrm{GeV}$. See Figure 2.6 to see where this cut occurs relative to the minimum bias cross section. The hardest $E_{T}$ triggers are biased relative to the Veto trigger. The differential cross section with respect to pseudorapidity 
$d E_{T} / d \eta$ is shifted to lower values of $\eta$. This bias is dramatically reduced for less central $E_{T}$ triggers.

Figure 2.6 shows the differential cross section $d \sigma / d E_{T}$ in barns/GeV as a function of $E_{T}$ for $200 \mathrm{GeV} /$ nucleon ${ }^{32} \mathrm{~S}$ projectiles incident upon a number of targets. The data were taken with a minimum bias trigger. For heavy targets, the dis-

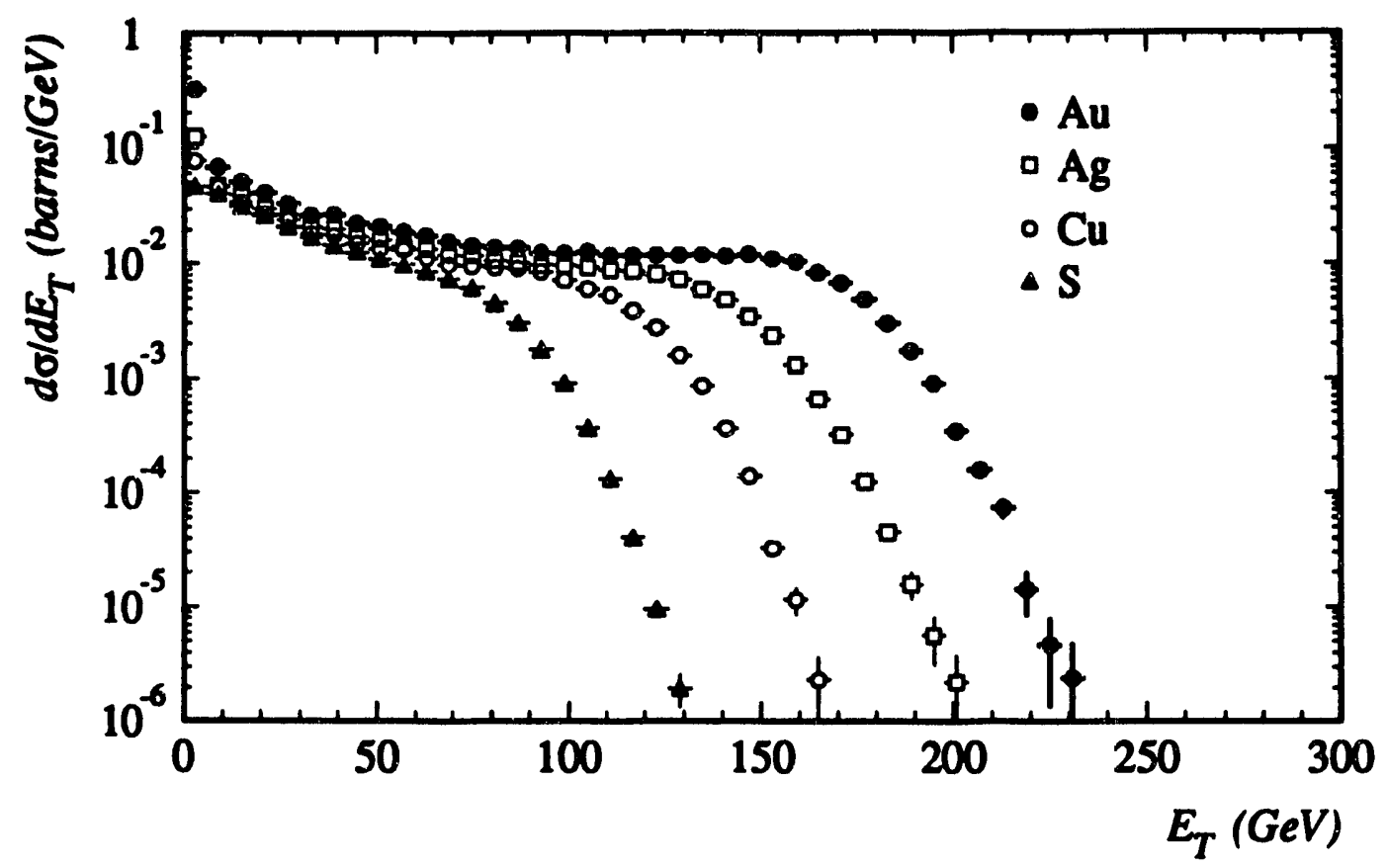

Figure 2.6: Minimum bias differential cross sections $d \sigma / d E_{T}$ as a function of $E_{T}$ for $200 \mathrm{GeV} /$ nucleon ${ }^{32} \mathrm{~S}$ projectiles. The characteristic shape for heavy targets-falloff at low $E_{T}$, broad plateau, sharp falloff at high $E_{T}$-is evidence for the geometrical interpretation.

tributions can be characterized by a rapidly falling cross section from peripheral collisions, a broad plateau while the incident nucleus "dives in" the larger target, followed by a sharp falloff at high $E_{T}$ from events which fluctuate about "average central collisions". For light targets the transition from peripheral to central collisions is less well defined-the projectile "passes by" rather than dives in. In ${ }^{32} \mathrm{~S}+{ }^{32} \mathrm{~S}$ collisions the observation of an $E_{V \text { eto }}$ signal for the highest $E_{T}$ events indicates there is a subset of peripherally interacting nucleons even in the most central collisions. This broad geometrical picture and the idea of average central collisions are supported by impact parameter-weighted convolutions of $p A E_{T}$ spectra [Rem88], [Mar91]. Figure 2.7, a scatter plot of $E_{T}$ versus $E_{V \text { eto }}$, shows the correlation between $E_{T}$ and $E_{V \text { eto }}$ for minimum bias ${ }^{32} \mathrm{~S}+\mathrm{Au}$ interactions at $200 \mathrm{GeV} /$ nucleon. The clear anticorrelation between transverse and forward energy provides more evidence for the geometrical interpretation outlined above. A similar, strong anticorrelation was reported between the mean charged multiplicity detected in the streamer chamber and $E_{V \text { eto }}$ in $200 \mathrm{GeV} /$ nucleon ${ }^{16} \mathrm{O}+\mathrm{Pb}$ collisions [Bam87]. The important point is that either an $E_{T}$ trigger, an $E_{V \text { eto }}$ 


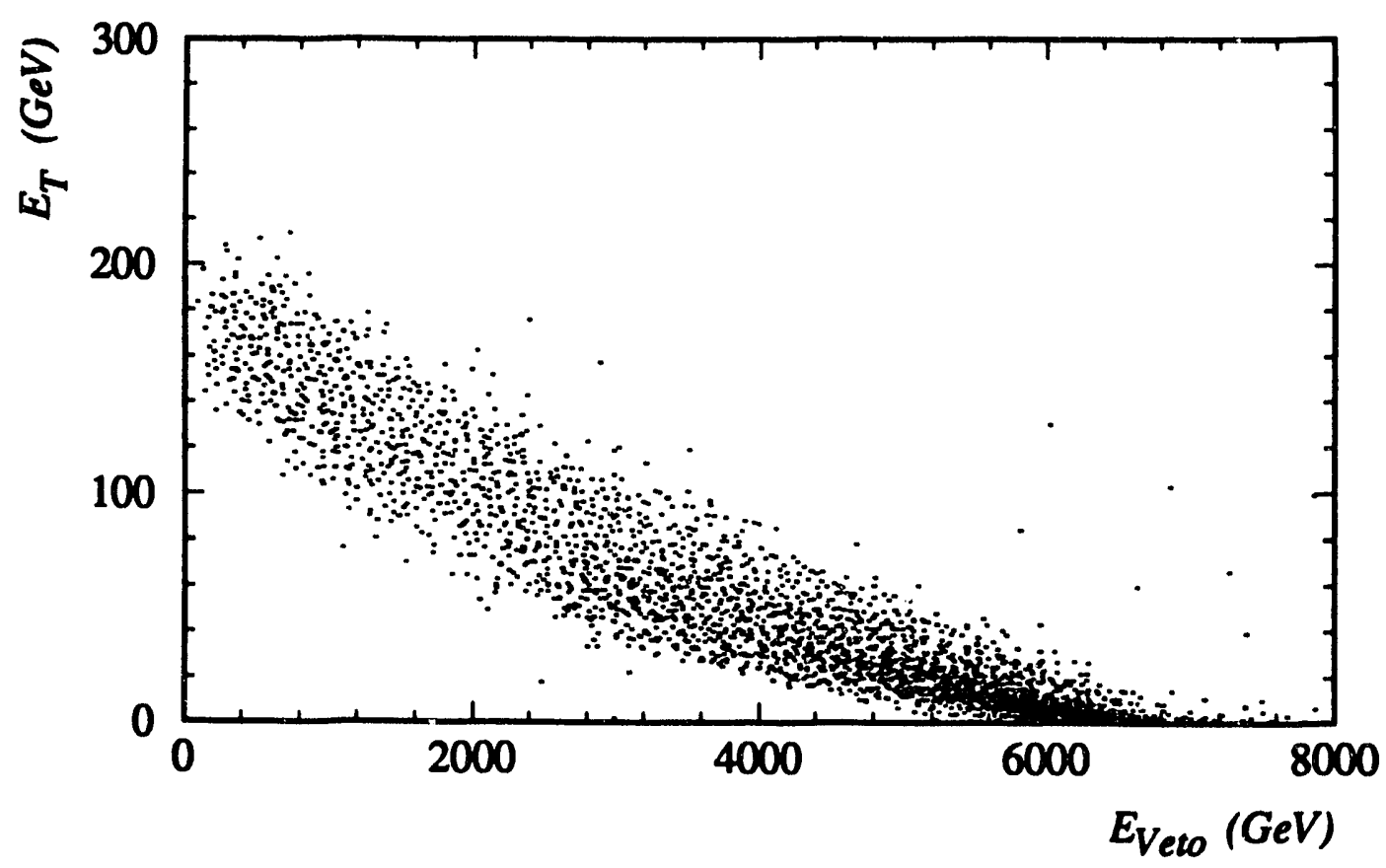

Figure 2.7: $E_{T}-E_{V \text { eto }}$ correlation for minimum bias ${ }^{32} \mathrm{~S}+\mathrm{Au}$ interactions at 200 $\mathrm{GeV} /$ nucleon. The strong anticorrelation prnvides more evidence for the geometrical interpretation.

trigger or a trigger derived from charged multiplicity, can be used to select violent, central collisions. This is the event class where both the energy density and the interaction volume may be large enough to produce a transition to quark matter. This event class is not rare. Events in the tail of the $E_{T}$ distribution still occur with appreciable probability at the 100 mbarn level. The NA35 analysis of rapidity and transverse momentum distributions of both charged and neutral strange particles has concentrated on the central event classes obtained from the Veto and $E_{T}$ triggers.

The CCD data acquisition recorded 5000 events for the hard Veto trigger, 2000 events for the hard $E_{T}$ trigger, and 4500 events for the medium $E_{T}$ trigger, all for ${ }^{32} \mathrm{~S}+{ }^{32} \mathrm{~S}$ collisions at $200 \mathrm{GeV} /$ nucleon. We elected to measure events from the medium $E_{T}$ data set because the multiplicity was not so high that it swamped the CCD resolution. Approximately 220 events have been measured and analyzed. We discuss track measuring in Section 4.4.3. Approximately 6000 events were recorded for heavier targets. A handful of events with $\mathrm{Cu}$ targets were measured to investigate the feasibility of analyzing heavy target data. Because of the higher charged particle multiplicities, the measuring demands increased and both the physics acceptance and efficiency decreased. As a result, we were unable to study charged particle spectra systematically as a function of target mass.

For the central triggers, the selection of streamer chamber events for measurement and analysis was straightforward. Events were scanned quickly, rejecting those which were too faint because of voltage fluctuations of the Marx generator, 
so obscured by spiralling electrons that they were deemed unmeasurable, or of such a low multiplicity that they cuuld only have resulted from a trigger inefficiency. Less than 10\% of CCD-imaged events were rejected according to these criteria and their exclusion from the data sample is believed not to introduce a bias. 


\section{Chapter 3}

\section{CCD Supervision System}

\subsection{Introduction}

In spite of the notable achievements of the UA5 collaboration [Aln87] at the SPS collider and its double streamer chamber ancestor at the ISR [Tho77] in the relatively low multiplicity environments of $p \bar{p}$ and $p p$ collisions, the physics analysis of streamer chamber pictures has been severely handicapped by the conventional method of data reduction, i.e., operator scanning and measurement. The measuring process is laborious, the resulting data samples have been small, and quantitative information residing in the image, for example, streamer brightness or the number of streamers per unit length, has generally been unused. Particle identification has only been possible for neutral particles, which can be well identified by their charged decays, and for low $\beta$, heavily-ionizing particles with laboratory momenta $p_{l a b}<1 \mathrm{GeV} / c$ [Eck73], although the feasibility of particle identification in the relativistic rise has been demonstrated [Eck77], [Bas81]. On :ie other hand, streamer chambers have nearly $100 \%$ tracking efficiency for charged particles, excellent two-track resolution, typically close to full $4 \pi$ acceptance in the center-of-mass, excellent momentum resolution, and employ well established track reconstruction techniques. The long term goals for the construction of an electronic camera system were to significantly accelerate the data reduction rate and to discriminate between primary particles of the same charge, for example $\pi^{+}, K^{+}$, $p$, without surrendering any of the features which make visual detectors so appealing. A directly digitized picture of a ${ }^{32} \mathrm{~S}+{ }^{32} \mathrm{~S}$ collision at $200 \mathrm{GeV} /$ nucleon in the NA35 streamir chamber, taken with the charge-coupled device (CCD) system described in this chapter, is shown in Figure 3.1.

The digitization of visual images is motivated by the existence of a powerful class of mathematical methods and image processing techniques which may be applied to digitized images to improve image quality, for pattern recognition, or to efficiently parametrize or represent an image in a way suitable for subsequent analysis. Direct digitization, the process of obtaining a gray-scale representation of a visual image without the intermediate step of capturing it on film, possesses additional advantages. Depending on the properties of the imager, a loss of information may be avoided, the spectral response and sensitivity may be extended, 


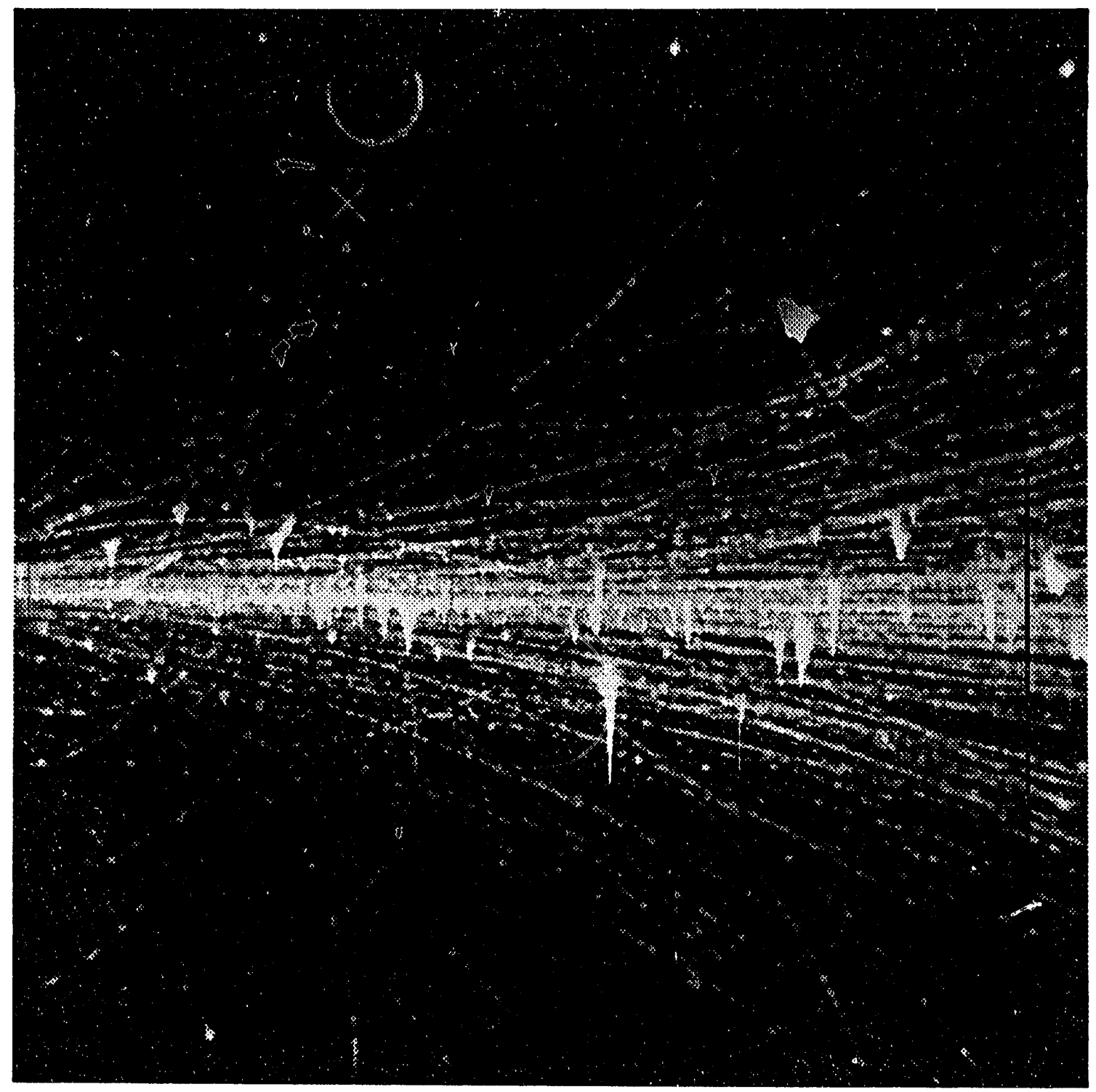

Figure 3.1: Directly digitized image of a ${ }^{32} \mathrm{~S}+{ }^{32} \mathrm{~S}$ collision at $200 \mathrm{GeV} /$ nucleon in the NA35 streamer chamber. The image consists of $1024 \times 1024$ picture elements (pixels). Each pixel contains up to 8 bits of intensity information. The grayscale image is obtained by defining a correspondence, a look-up table, between an intensity value and a shade of gray. 
and the resolution may be improved. The nearly real-time, continuous availability of images may also be of crucial importance.

Charge-coupled devices (CCDs) possess a number of attributes which make them ideal imagers for direct digitization [Jan85]. They have a wide spectral bandwidth, broad dynamic range, excellent sensitivity, and exceptional linearity. However, even the highest density pixel arrays available in 1985 did not have a resolution comparable to filn. Moreover they were expensive, not readily attainable, and virtually unreplaceable, since the only two manufacturers of large devices abandoned the off-the-shelf, commercial market at about this time. In addition, cheap, high-speed, high-density recording devices and wide bandwidth data buses were still in their infancy making data acquisition and recording problematic.

In spite of these difficulties, the CCD Supervision System was developed, so named because its first intended application was in a supervisory role. Another CCD system for streamer chamber imaging was being developed by a group from Michigan State University (MSU) [Ang88] but it utilized less dense CCD chips. Although adequate for the MSU group objective of studying relatively low multiplicity, intermediate energy collisions well below the pion production threshold $p_{\text {lab }}=0.792 \mathrm{GeV} / c$, these chips lacked the resolution required to image the extremel:- high multiplicity events anticipated at $p_{l a b}=200 \mathrm{GeV} / c$.

Based on the physics objectives of NA35, a rather elaborate "wish list" of desired system properties and functions was devised [GSI85]. The most important performance requirements were the highest possible image resolution, good sensitivity, fast readout, high speed pixel processing power, and faithful graphical representation of the CCD images at the full resolution of the devices. High resolution dictated using the largest available CCD, the Texas Instruments $1024 \times 1024$ pixel Virtual Phase array. Sufficient sensitivity was obtained by building a high gain, two-stage image intensifier optical assembly. Fast readout (2 s to read three $1024 \times 1024$ pixel $\times 8$ bit images) was accomplished by a combination of measures: the camera drive electronics and analog-to-digital converters (ADC's) were tuned to achieve exceptionally fast pixel transfers, an interface board was designed and built which multiplexed the three camera data streams at their full rate, and a fast array processor was employed to fetch the pictures from the interface and buffer them in memory, again at the full rate which any single camera could deliver. The array processor also provided the computing power for full frame, image processing operations and a convenient data path to the host computer. A graphics computer, with enough resolution in both pixels and intensity to permit a one-to-one mapping of CCD images, served as the display. ${ }^{1}$

This chapter will describe the CCD Supervision System hardware, the software developed for image acquisition and three-view data recording, and the optical configurations employed in experiment NA35. ${ }^{2}$ It will conclude with a brief discussion

\footnotetext{
${ }^{1}$ We particularly thank R. Dwinell and M. Fahmie of the LBL Bevatron Electronics Group for their help with the design and construction of the custom interface board, H. Fessler, K. Geissler and J.P. Fabre for advice and assistance in the design of the image intensifier optical assemblies, and $M$. Wensveen and $H$. Ellison for the construction of the optical assemblies.

${ }^{2}$ Some of the material presented in this chapter has been published in Ref. Tin87.
} 
of system performance as an online monitor during the Fall 1986 running period with ${ }^{16} \mathrm{O}$ beams when only two cameras were instrumented. A third camera ${ }^{3}$ was added for the Fall 1987 run with ${ }^{32} \mathrm{~S}$ beams. The next chapter will describe techniques for full three-view data reduction and momentum reconstruction.

\subsection{CCD Supervision System Hardware}

A block diagram of the Supervision System, illustrating the five major hardware subsystems, is presented in Figure 3.2. Light from the streamer chamber is amplified in the image intensifier assembly, imaged onto the CCDs, and read out through the interface into the array processor, where it is buffered while being read into the main computer memory. The implementation is based on a VAXstation II computer with the array processor and the display configured as peripheral devices on its bus. Once in memory, an image is available for display, interactive examination, background calculation and recording to tape. Two other schemes were investigated [Tin87] and rejected during the design phase.

\subsubsection{Charge-Coupled Devices}

CCDs were first developed by Bell Laboratories in 1970 in the form of a linear shift register [Boy70]. The ability of silicon to detect visible radiation caused CCDs to be recognized as potential imaging sensors [Jan85]. They have developed rapidly, motivated principally by the desire to construct a fully TV-compatible, all-solid-state imager on a single chip, and are now among the largest metal-oxide semiconductor (MOS) devices being fabricated [Blo83]. They have been used in a large number of scientific applications including ground-based astronomical observatories and as image transducers for NASA's Galileo-Jupiter Orbiter [Jan81], the Solar-A experiment [Hov89], and for the Wide Field/Planetary Camera on the Hubble Space Telescope. The depletion layers of CCDs are also the thinnest silicon samples in which the ionization energy loss of minimum ionizing particles has been detected [Dam86]. Combined with their small pixel size and intrinsic two-dimensional information, this makes them attractive candidates for high resolution vertex detectors [Ake87]. For completeness, a brief discussion of how CCDs are made and how they work follows. A more complete general discussion, from which this synopsis is drawn, including the physical principles that underlie the operation of all silicon devices, can be found in Ref. Dam86.

The basic principle employed by all semiconductor detectors is the detection of charge generated within the depletion region of a reverse-biased pn junction. Figure 3.3 shows a schematic illustration of both the depth structure and the image area of the virtual phase CCD used in our application. A thin, epitaxial layer $(10-20 \mu \mathrm{m})$ of p-type silicon is grown on a suitable substrate. A pn junction is constructed by introducing a shallow implant of $n$-type dopant, the $n$-channel. The

\footnotetext{
${ }^{3}$ Our third CCD chip was obtained courtesy of the Jet Propulsion Laboratory with the assiotance of J. Janesick of JPL.
} 
CCD CAMERAS

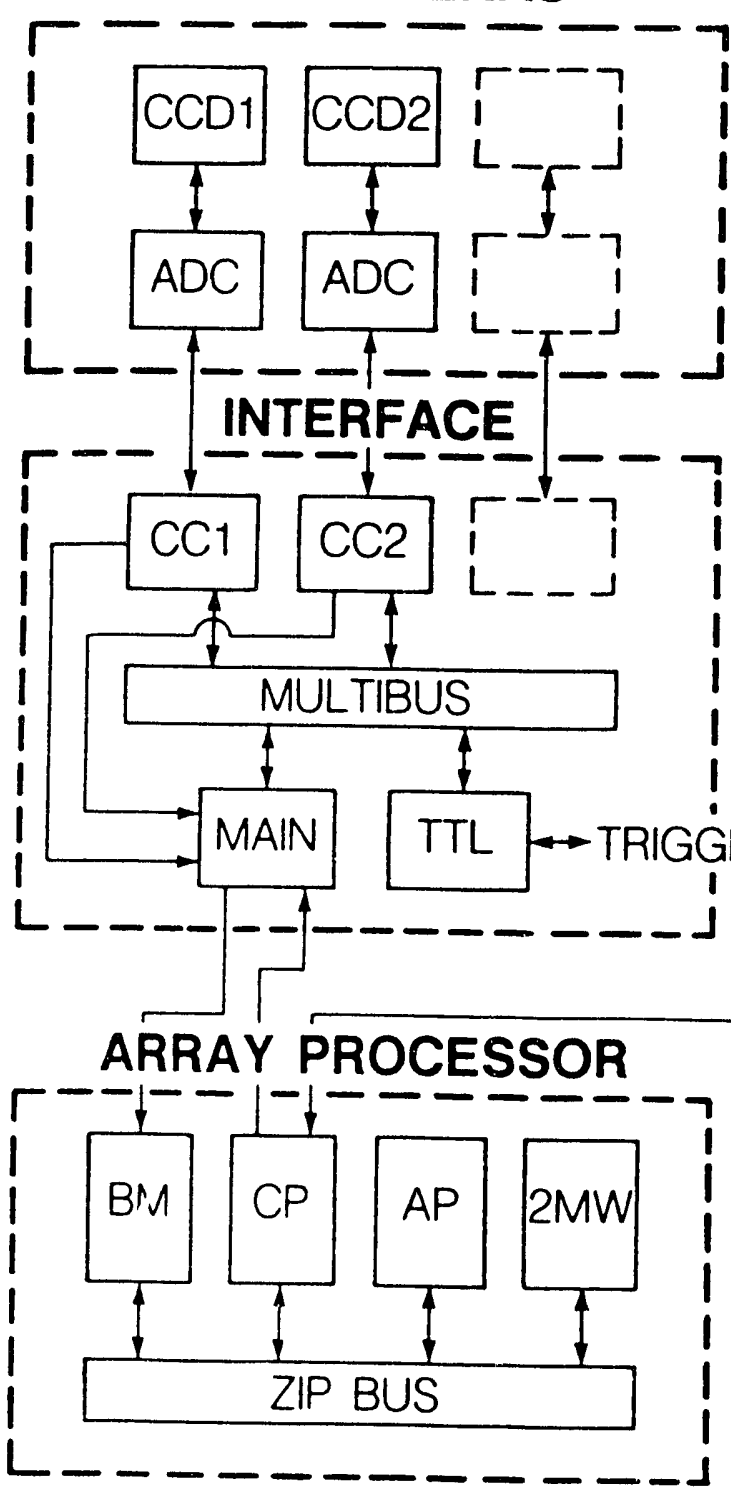

COLOR DISPLAY

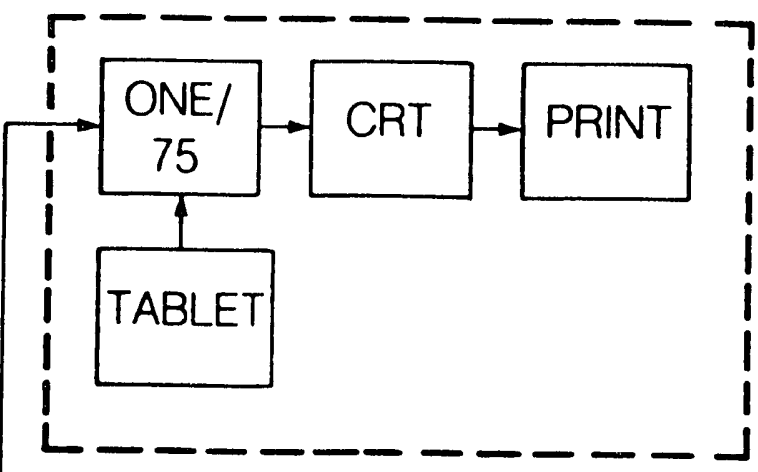

HOST COMPUTER

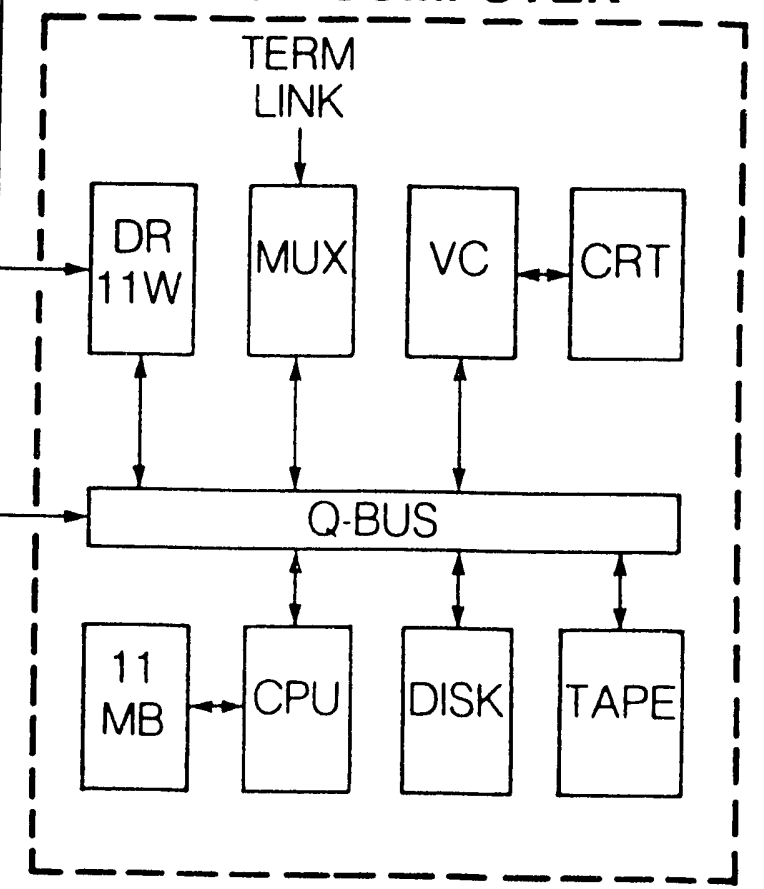

Figure 3.2: CCD Supervision System hardware. The major components are the image intensifier optical assemblies, the CCD cameras, the interface, the array processor, the color display, and the host computer. 

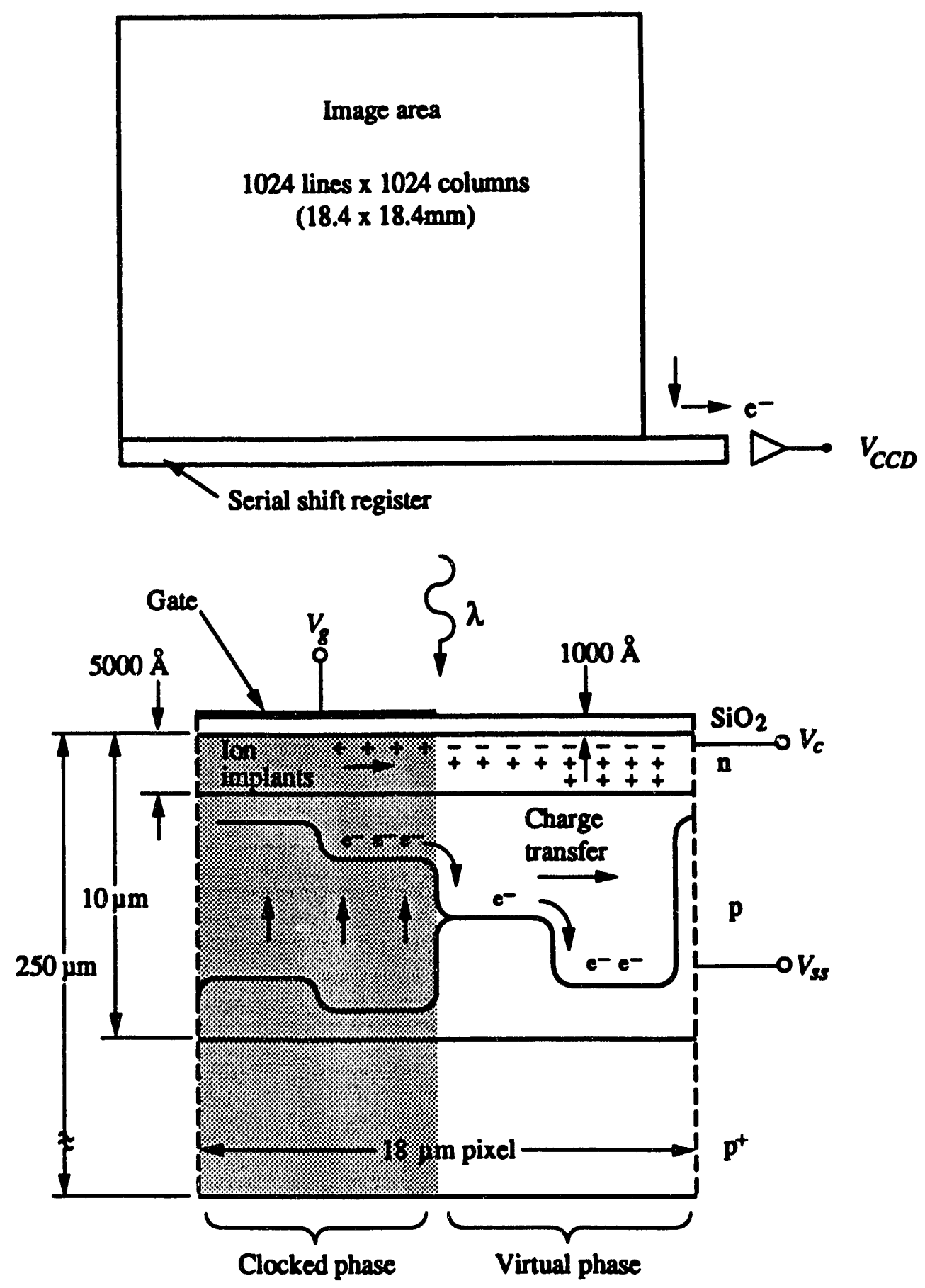

Figure 3.3: Texas Instruments Virtual Phase CCD. The device is frontside illuminated, indicated by the incident photon. Note that the gate electrode only covers half the pixel, dividing it into clocked and virtual regions. When the gate potential is low, charge can be collected in the potential wells of either region. Charge can be transferred from the clocked to the virtual region by raising the gate potential above the potential of the virtual barrier, shown schematically by the arrows. 
surface above the channel is oxidized to form an insulating layer on top of which is applied a conducting gate. Because of diffusion of electrons into the $p$ material and holes into the n material, a small depletion region devoid of free charge carriers develops at the interface between the two kinds of silicon, the characteristic feature of the pn junction. Different bias voltages can be applied to the gate, the channel and the substrate, $V_{g}, V_{c}$, and $V_{g}$, respectively. By reverse-biasing the junction, i.e., $V_{c}>V_{o s}$, the depletion region can be extended. By maintaining the gate at a lower potential than the channel, $V_{s}<V_{c}$, a potential energy minimum can be created within the totally depleted $\mathbf{n}$ channel. Electrons liberated by the passage of a particle accumulate in this buried channel. This is the basic mechanism for storing charge in CCDs.

Two more steps must be taken to create the two-dimensional pixel structure of an imaging CCD and provide a method for readout. These are depicted schematically in Figure 3.4. Channel stops, typically heavily doped p-type implants ap-

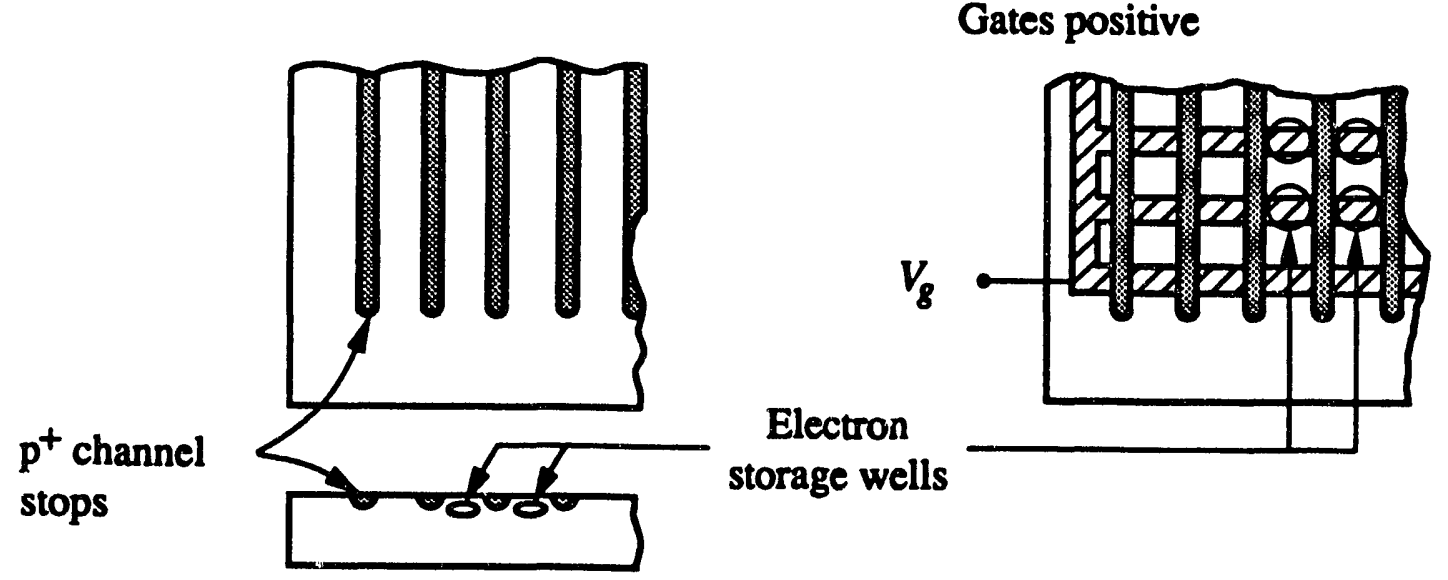

Figure 3.4: Creation of two-dimensional imaging CCD by the introduction of channel stops and gates.

proximately $1 \mu \mathrm{m}$ wide and deep which become fully depleted as part of the overall biasing of the CCD, are strips of negative space charge which confine electrons in the horizontal or serial direction. Charge confinement in the vertical or parallel direction is achieved by building a non-uniform gate structure consisting of a series of horizontal bars. These are biased positively to form an array of potential wells below the gates and in between the channel stops. Each member of this array of wells constitutes a pixel. A more complicated gate structure is required to move charge. By clocking the CCD-applying properly synchronized, time-varying gate voltages-charge can be transferred in the vertical direction. The virtual phase technology [Hyn79] employs a single level gate structure, covering only half of the pixel, which enables the device to be frontside illuminated and still achieve acceptable quantum efficiency. The edge of the gate divides the pixel into clocked and virtual regions. The four-level potential structure shown in Figure 3.3 is constructed by 
introducing additional n- and p-type ion implants at different positions and depths in the buried channel. These are indicated by plus and minus signs in Figure 3.3. Charge is transferred from the clocked to the virtual region, shown schematically in the figure by the motion of the electrons from the well under the clocked phase to the well under the virtual phase, by changing the gate voltage so the potential of the clocked well is higher than the potential of the virtual barrier. Depending on the biasing, charge collection can occur in the potential well in either the clocked or virtual region.

Below the imaging CCD is one additional row of pixels, a linear CCD with its own gate structure. The imaging CCD is clocked one row at a time in the parallel direction into this serial shift register. Then the charge is shifted one pixel at a time in the serial direction. The charge on the last pixel is transferred onto the input of a transistor located on the chip. The voltage change induced at the output of the transistor drives an amplifier mounted on the chip. The analog voltage at the output of this on-board amplifier $V_{O C D}$ is proportional to the charge generated in the pixel by the passage of a photon or ionizing particle and constitutes the signal of the CCD. The serial and parallel charge transfers can be accomplished at high rates (3-10 $\mathrm{MHz}$ ) with high efficiency. CCDs acquire their reputation for being slow devices because of the relatively long time it takes to sample the signal in order to perform analog-to-digital conversion with acceptably low noise.

The performance of CCDs as imaging transducers can be divided into three areas: spectral response and efficiency of primary photon-electron conversion, resolution capability, and signal quality. A good in-depth discussion of CCD performance in scientific applications including a description of how these performance characteristics are measured can be found in Ref. Jan85.

The spectral response of CCDs to incident photons is characterized by their wavelength-dependent quantum efficiency (QE), which measures the probability of generating an electron per incident photon per pixel. It depends primarily on the depth of the photosensitive volume of the CCD, on surface effects at the boundaries of the photosensitive volume, and for the case of frontside illuminated CCDs on absorption effects in the gate structure. Since far-ultraviolet and X-ray photons can generate more than one electron-hole pair in silicon, the QE can be greater than 1. For the near-infrared through the visible, photons which interact in the photosensitive volume generate one electron-hole pair. Depending on the device, QEs in the visible part of the spectrum range from $20 \%$ to nearly $100 \%$ peaking at wavelengths from $\sim 600-800 \mathrm{~nm}$ which is well tuned to the characteristic $630 \mathrm{~nm}$ orange light in a He-Ne streamer chamber. Although the statistical probability of detecting single photons is high, the ultimate sensitivity of a CCD is limited by noise.

The resolution capability of a CCD is determined by the extent to which charge generated by photons incident on a given pixel is collected by that pixel and retained by that pixel during the numerous charge transfers required for readout. The apparent charge collection ability depends on two factors: charge diffusion and charge transfer efficiency (CTE). CTE affects the output signal by diverting charge trom the target pixel [Jan85]. The CTE for modern CCDs is consistently 
greater than .9995 in both the parallel and serial directions and can be assumed not to degrade an image, particularly when the signal level is greater than $\sim 1000$ $e^{-}$per pixel. A quantitative measure of the resolution of an imager, and hence of the impact of diffusion on the charge collection efficiency of a CCD, is its modulation transfer function (MTF). The MTF describes the output response of an imager to sinusoidal input intensity variations as a function of spatial frequency. The ideal MTF of discrete sampling devices like the CCD [Blo83] is $\sin (x) / x$ where $x=\pi f / 2 f_{\max }, f$ is the spatial frequency of the input intensity distribution, and $f_{\max }$ is the maximum spatial frequency a device can resolve. The resolution of an optical instrument is often stated in line-pairs(lp)/mm at $50 \%$ MTF. An ideal CCD would achieve an MTF of $64 \%$ at $f=f_{\max }=1 / 2 d$, where $d$ is the size of a pixel in mm. The theoretical model of Crowell and Labuda [Cro69] has been successful in accounting for the departure of CCD resolution from ideal behavior. The film used in the NA35 filn cameras had a measured resolution of $60 \mathrm{lp} / \mathrm{mm}$ at $40 \%$ MTF [Wen90].

CCD signal quality can be characterized by sensitivity, dynamic range, and linearity. As mentioned above, CCD sensitivity is ultimately determined by noise. An intrinsic source of noise is the dark current, the signal generated in the absence of illumination. Dark current is important in astronomical applications when integration times are long and light levels can be extremely low. In the accelerator context, where integration times are short, dark current is generally negligible. Operated at $\sim-45^{\circ} \mathrm{C}$, triggered on the first interaction in the $4 \mathrm{~s}$ extraction spill, and exposed for $\simeq 700 \mathrm{~ms}$, the dark current contribution to our signal is less than $10 e^{-}$per pixel. ${ }^{4}$ A second source of noise is the on-chip amplifier which contributes $\sim 10-50 e^{-}$rms. For virtual phase CCDs, however, the noise floor is always dominated by spurious charge generated during clocking, which introduces $\sim 200 e^{-}$noise. The dynamic range is determined from the pixel full well capacity, the maximum charge signal that can be collected, and the noise floor. The full well capacity of CCDs ranges from 50,000-200,000 $e^{-}$. If the charge deposited in a pixel exceeds the full well capacity, the signal spills or blooms into neighboring pixels, undermining the resolution. Blooming is exacerbated by charge transfer so the bloomed regions of a CCD image appear to "drip" in the direction of parallel shift. CCDs are exceptionally linear devices, exhibiting nonlinearities of less than $1 \%$ over their full dynamic range from full well down into the noise.

The $1024 \times 1024$ pixel Texas Instruments virtual phase CCDs employed in the Supervision System had a sensitive area of $18 \times 18 \mathrm{~mm}^{2}$ and were essentially devoid of defects. Their QE ranged from $20-50 \%$ for visible wavelengths from $\sim 350-850$ $\mathrm{nm}$. They achieved an MTF of $\simeq 50 \%$ at $f_{\max }=1 /(2 \times 18 \mu \mathrm{m}) \simeq 28 \mathrm{lp} / \mathrm{mm}$ with a CTE of better than .9997 for both horizontal and vertical charge transfers. With a noise floor of $\sim 100-200 e^{-}$from spurious charge and a full well capacity of $\sim 100,000 e^{-}$, the dynamic range of the device was $\sim 500$.

Compared to film, CCDs have both advantages and disadvantages. Assum-

\footnotetext{
${ }^{4}$ Precise specifications for the Texas Instruments virtual phase CCDs used in the Supervision System were provided by J. Janesick from Jet Propulsion Laboratory internal reports [Jan90].
} 
ing that the $\sim 7 \mu \mathrm{m}$ silver-halide film grain plays the same sampling role as a CCD pixel, the limiting resolution of film would occur at a spatial frequency $f_{\max }=1 /(2 \times 7 \mu \mathrm{m}) \simeq 70 \mathrm{lp} / \mathrm{mm}$, more than a factor of 2 better than the most dense CCD arrays. Furthermore, images recorded on standard $35 \mathrm{~mm}$ or $70 \mathrm{~mm}$ film require less demagnification than comparably sized images recorded on single CCDs. This means that CCDs are forced to resolve scenes at spatial frequencies closer to their limiting resolution. For example, the NA35 film cameras, operating at demagnification $M=30$, image a $1 \mathrm{~mm}$ diameter streamer over $\sim 25$ film grains, whereas the CCD cameras, operating at $M=65$, image the same streamer onto a single pixel. Film affords faster capture and more compact data storage of high resolution images than current digital technology can offer. Mechanical film transports have been devised so that film cameras can achieve the full event rate permitted by the timescale for generation of the streamer chamber high voltage pulse. However, CCDs are more sensitive than film, often by factors of 10 or more, depending on the CCD noise floor and the film grain exposure threshold. And their response is linear, compared to the logarithmic response of film, which is important for applications which need to extract information from the intensity distributions, not just the positions, of luminous objects. The main advantage of CCDs is that they provide proportional, directly digitized, high intensity resolution, gray-level representations of visual data.

\subsubsection{Cameras and Controllers}

The camera systems were manufactured by Photometrics Ltd., of Tucson, Arizona. Each consists of a camera head with optics, CCD, and cooling system; a camera electronics box that regulates the CCD voltages, drives the CCD clock phases, and reads and digitizes the analog pixel charge signal; and a camera controller board which administrates camera functions.

The camera heads provide the mechanical mounting for the CCDs, coupling the optics, cooling and electronics. The CCDs are cooled to a temperature of $-45^{\circ} \mathrm{C}$ by a combination thermoelectric (Peltier) and flowing water system. The camera heads include mounts for standard Pentax lenses. Electronically controlled mechanical shutters open and close in $\sim 10$ to $40 \mathrm{~ms}$.

The camera electronics are housed in individual boxes close to each camera head. They drive the CCDs' clock phases at $500 \mathrm{kHz}$, leading to a serial transfer rate of $2.0 \mu \mathrm{s} / \mathrm{pixel}$. The CCDs are read out in a steady, noninterruptible stream of pixels, each digitized to 9 bits by successive approximation ADCs. The 9 bit digitization was a compromise between the need for gray-level sensitivity and the requirement of fast readout. The digitized pixel data are transmitted serially over a $25 \mathrm{~m}$ cable from the experimental area to the electronics room. Power and control signals share the same cable, and the drive voltages are regulated in the electronics boxes to minimize noise at the CCDs.

The cameras are controlled by single-slot Multibus boards, which use standard Multibus communication protocol. The camera controllers supply the following functions: definition of CCD array subsections for individual readout, readout of 
the CCD array, continuous clearing of CCD pixels, shutter operation including timed exposure, amplifier gain adjustment, and summation of pixels into lower resolution images. The digitized pixel data appear on an auxiliary connector at the front edge of each board. In this application the Multibus DMA bus-master control circuitry was disabled on each board so pixel streams from multiple controllers could be transferred in parallel to the main interface board.

\subsubsection{Image Intensifier Optical Assemblies}

In spite of the superior photon sensitivity of CCDs, the faint avalanche/streamers in the NA35 chamber could not be imaged directly. An avalanche containing $10^{8}$ electrons, in a chamber operating at atmospheric pressure in an electric field of $\sim 20 \mathrm{kV} / \mathrm{cm}$, isotropically emits $\sim 10^{8}$ photons [Bul67]. Because of the small aperture of a $\sim 1 \mathrm{~cm}^{2}$ lens located $\simeq 330 \mathrm{~cm}$ from this light source, only $\sim 10^{3}$ photons would be collected by a typical camera system. Distributed over the $\sim 3 \times 3$ pixels required to achieve optimum resolution, only 100 photons strike each pixel. Folding in the quantum efficiency, $\sim 30 e^{-}$would be collected in each pixel, much lower than the 100-200 $e^{-}$read noise of the CCD [Jan90]. With a full well capacity of $\sim 100,000 e^{-}$, a gain of $\sim 1000$ would push an average avalanche/streamer into the heart of the CCD dynamic range, i.e, 30,000 $e^{-}$. During a test run, a provisional setup with three stages of image intensification indicated the required light amplification and led to the development of an extremely high gain, two-stage image intensifier optical assembly. In turn, the availability of a very sensitive imaging system led to the optimization of the BEVALAC streamer chamber which facilitated high multiplicity experiments with heavy targets and projectiles [Cha92].

The optical assembly, including a mounted CCD camera and a mirror housing, is shown in Figure 3.5. Two of the three CCD camera systems required mirrors because the small camera pavilion of NA35 could only accommodate both film and CCD imaging systems by squeezing the CCD assemblies into indirect lines-of-sight. Viewing reflected light from the mirror was an advantage because it distanced the optical assembly from the camera hole where the streamer chamber magnetic field leaked through the shielding plate. As a result, no compensation coils were necessary and the introduction of additional optical distortion was avoided. Tilting the mirror changed the field of view. The third camera, pointing directly down into its camera port, needed a compensation coil to counteract the leakage field.

Reflected light from the chamber is imaged by the primary lens onto the photocathode of the first stage image intensifier, a second generation, inverter type tube with incorporated microchannel plate (MCP). Some of the characteristics of the intensifiers are listed in Table 3.1. Different primary lenses can be utilized to change the demagnification, the field of view, and the maximum aperture for light collection. The MCP intensifier is screwed into a cylindrical delrin collar, which serves as the insulator for the high voltage second stage, then encased in an aluminum cover which supports the lens mount. The output phosphor of the first stage is proximity-coupled directly to the photocathode of the second stage intensifier, a first generation, inverting, electrostatic-focusing tube. A small layer 


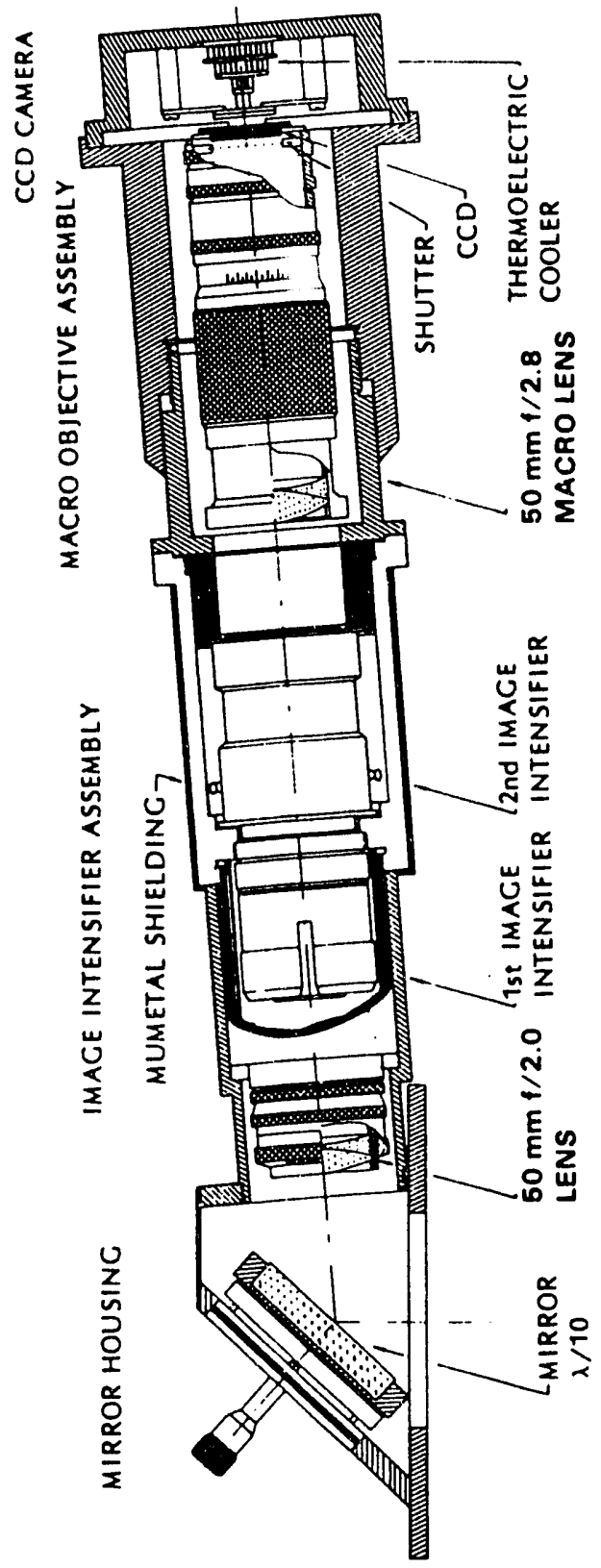

Figure 3.5: Two-stage image intensifier optical assembly. Incident light is reflected by the mirror and focused by the primary lens onto the photocathode of the first stage intensifier. The intensifiers are proximity-coupled with optical grease. The image on the output phosphor of the second stage is focused onto the CCD at demagnification $M=1$. 


\begin{tabular}{||l|l||}
\hline \hline First Stage & Varo model 3603-1 or AEG model XX 1400 \\
\hline Type & $\begin{array}{l}\text { Second generation, inverter with incorporated mi- } \\
\text { crochannel plate (MCP) }\end{array}$ \\
\hline Focussing method & $\begin{array}{l}\text { Electrostatic from photocathode to MCP input, } \\
\text { proximity from MCP output to phosphor }\end{array}$ \\
\hline Sensitive diameter & $25 \mathrm{~mm}$ \\
\hline Gain & 60,000 \\
\hline Limiting resolution & $34 \mathrm{lp} / \mathrm{mm}$ \\
\hline Resolution at $50 \%$ MTF & $9.7 \mathrm{lp} / \mathrm{mm}$ \\
\hline Distortion & $5 \% \mathrm{maximum}$ \\
\hline Nominal operating voltage & $2.65 \mathrm{~V}$ \\
\hline
\end{tabular}

\begin{tabular}{||l|l||}
\hline Second Stage & Varo model 1248-1 \\
\hline Type & First generation, inverter \\
\hline Focussing method & Electrostatic from photocathode to phosphor \\
\hline Sensitive diameter & $40 \mathrm{~mm}^{6}$ \\
\hline Gain & 100 \\
\hline Limiting resolution & $56 \mathrm{lp} / \mathrm{mm}$ \\
\hline Resolution at $50 \% \mathrm{MTF}$ & $18.6 \mathrm{lp} / \mathrm{mm}$ \\
\hline Distortion & $5 \% \mathrm{maximum}$ \\
\hline Nominal operating voltage & $15 \mathrm{kV}$ \\
\hline \hline
\end{tabular}

Table 3.1: Characteristics of first and second stage image intensifier tubes. 
of optically conducting grease provides the seal. The second stage tube is inserted into the delrin collar, pressed into place to make optical contact, and held in place by a delrin barrel. Both intensifiers are sheathed in thin, mu-metal jackets to shield the devices from small stray fields leaking through the shielding plate. A small hole is left in the side of the assembly for the power cables. The amplified, erect image on the output phosphor of the second stage is lens-coupled to the CCD by a $50 \mathrm{~mm}$ focal length, Macro, relay lens at a demagnification $M=1$. The relay lens is housed in an aluminum barrel with a threaded section for fine focusing. The focusing barrel was machined so that one turn moved the lens one mm towards or away from the output phosphor. The final focus is determined with the aid of a resolution chart and is sensitive to $\sim 1 / 4$ of a turn, $250 \mu \mathrm{m}$. The CCD image sensor is positioned at the focal plane instead of film.

The order of the intensifiers in the optical assembly is important. The electrostatic tube is linear throughout its operating range. An automatic brightness control is incorporated in the MCP tube which limits the output luminosity at high input light levels. The response of this tube, according to the manufacturer's technical description, is shown in Figure 3.6. By adjusting the gain control of the

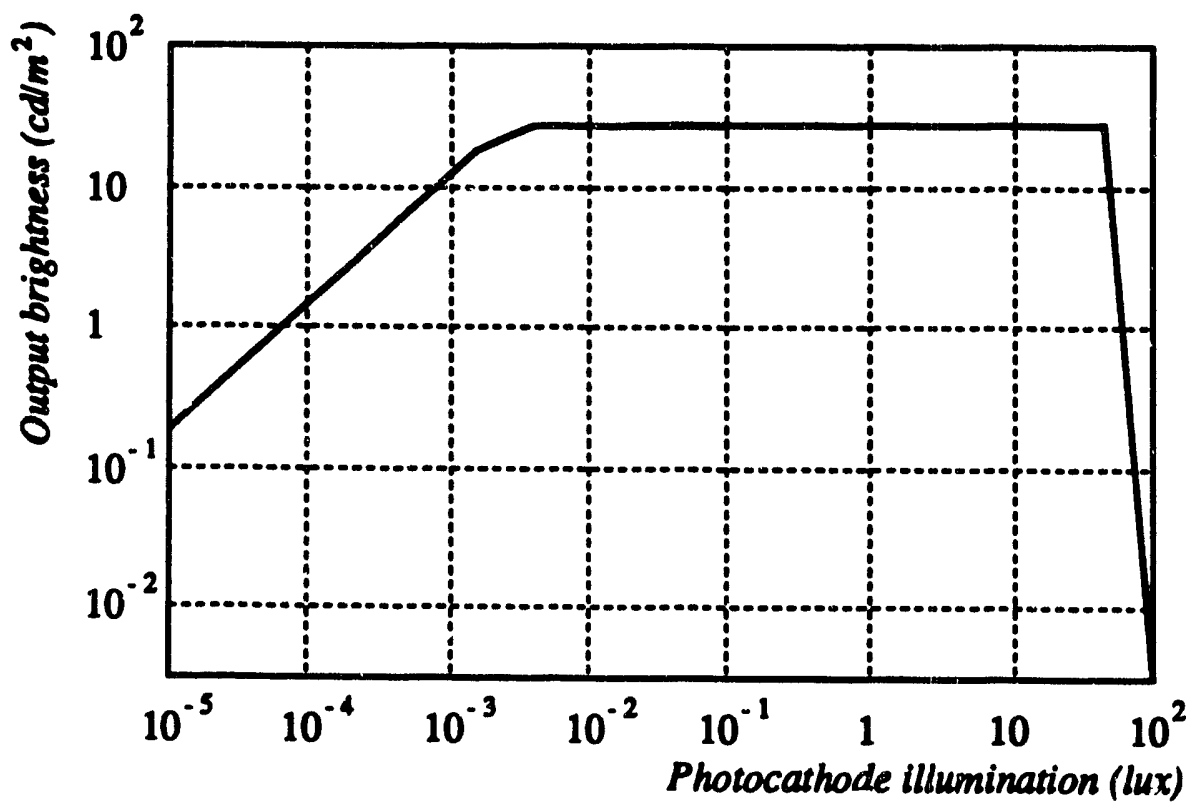

Figure 3.6: Response curve of MCP image intensifier.

MCP intensifier, the flares can be pushed into the saturation region of the gain curve, indicated in Figure 3.6 by the horizontal line for illuminations greater than

\footnotetext{
${ }^{6}$ The limiting resolution was measured with the USAF 1951 resolving power target, which locally generates a squarewave pattern of $\sim 100 \%$ contrast. The resolution at $50 \%$ MTF was estimated by interpolating from the manufacturer's specifications at 60 and $20 \%$ MTF.

${ }^{6}$ Because the two intensifier stages are proximity coupled only $25 \mathrm{~mm}$ of the sensitive area of the second stage is illuminated.
} 
$\simeq 10^{-2}$ lux. An intensity difference of 100-1000 at the input, relative to a flare irradiating the photocathode at $10^{-1}$ lux, can be reduced to a difference of 3-15 at the output, resulting in an apparent suppression of bright flares and reduced blooming. Some blooming can be observed dripping upward in Figure 3.1 due to very bright flares in the chamber.

The maximum luminous gain of the two-stage intensifier setup is approximately $3 \times 10^{6}$. The extremely high overall gain was necessitated by the decision to lenscouple the image from the intensifier output phosphor to the CCD. Due to the finite aperture and response of the secondary lens only $0.3 \%$ of the light from the output of the image intensifiers was transmitted to the CCD. The film camera system used by NA35, borrowed and adapted from the UA5 experiment, employed image intensifiers with a luminous gain of $\sim 2000$. Images were transmitted directly to the film by pressing it against a fiber optic block which was proximity-coupled to the intensifier output phosphor. Mounting a fiber optic block directly onto the CCD front face and proximity-coupling the image from phosphor to fiber optic would have eliminated the need for the second stage intensifier and improved the resolution. This procedure risked damaging the CCD (our supply was 2-3 and unreplaceable!) and was never seriously considered. The limiting resolution of the optical system was $\sim 22 \mathrm{lp} / \mathrm{mm}$, determined directly by observing the last resolved image from a resolving power target. The dominant contributions were from the resolution of the MCP intensifier tube and from the $28 \mathrm{lp} / \mathrm{mm}$ physical pixil size of the CCD. The image intensifiers introduced non-symmetric distortion which increased with distance from the tube center. At extremely bright input levels, the space charge developed in the electrostatic-focusing section of one of the tubes altered the electron optics, leading to dramatic, event-dependent distortions. This was not an issue at CERN since the charge of the ion beams was low and the targets were located outside of the chamber. For experiments at the BEVALAC, utilizing heavily-ionizing $A u$ beams $(Z=79)$ and internal targets, this distortion had a significant impact on the goodness-of-fit and the efficiency of the momentum reconstruction. The maxima of the streamer intensity distributions produced from $2 \times 10^{4}$ to $1 \times 10^{5} e^{-}$per CCD pixel, with a detection threshold of $\simeq 200 e^{-}$, corresponding to an avalanche containing $\sim 10^{6}$ electrons.

\subsubsection{Host Computer and Array Processor}

The host computer for both program development and data acquisition is a DEC VAXstation II ( $\mu$ VAX II) running the VAX/VMS operating system. The computer was configured with a large internal memory because 10 Mbytes were reserved for CCD images. Since each full resolution, three-view event required $3 \times 1024 \times 1024=$ 3 Mbytes of data memory, two events could be buffered in memory for longer timescale processing while the current event was read into the system for display. Because of the large number of peripheral devices configured on the system, a large enclosure with $12 \mathrm{Q}$-bus slots was selected. The bus was subsequently expanded to 20 slots. The I/O devices for writing the large CCD images to tape evolved with the available technology from a slow streaming cartridge tape to two higher capacity, 
faster VHS video tape systems to up to four $8 \mathrm{~mm}$ Exabyte devices. During data acquisition the $\mathrm{I} / \mathrm{O}$ capability required to service multiple $8 \mathrm{~mm}$ tape drives and the picture stream from the cameras simultaneously exceeded the bandwidth of the Q-bus, which was the limiting factor for our data rate.

The array processor is a ZIP 3216 manufactured by Mercury Computer Systems. It is a six-board set that resides on the $\mu$ VAX Q-bus. The ZIP provides the system with data acquisition control, data buffering and high speed computing power for full-frame image processing operations. It executes at $9 \mathrm{MIPs}$, features concurrent control and arithmetic processors, base program memory and was configured with $3 \mathrm{Mwords}$ of 16 bit data memory to store CCD images. The array processor has $4.5 \mathrm{MHz}, 16$ bit, auxiliary input and output ports separate from the ZIP Q-bus interface to the $\mu$ VAX. The auxiliary $I / O$ ports are the entry point of CCD pixel data into the computer system. Programs executing in the array processor issue camera control commands, fetch the pictures from the $1 / 0$ ports and buffer them in data memory. The data are then DMA transferred over the Q-bus to the host computer at $\simeq 1 \mathrm{Mbyte} / \mathrm{s}$. The array processor has a library of standard image processing algorithms, its own programming language, and a hardware simulator program on the VAX for application development.

\subsubsection{Interface}

The CCD-array processor interface is a pair of Multibus boards that route signals between the external apparatus and the computer system. Both boards were designed and built at LBL. The TTL logic board sends and receives trigger signals to and from the experimental trigger electronics. The main interface board multiplexes these signal sources into the array processor $\mathrm{I} / \mathrm{O}$ ports.

The main interface board is the critical link in the data path between the CCDs and the host computer. It is a single Multibus card that communicates with the CCD camera controllers via both the Multibus and auxiliary data cables, and with the TTL logic board via the Multibus. It connects to the ZIP array processor I/O ports by a pair of cables. Its architecture is discussed in Ref. Tin87. The primary job of the main board is to multiplex the streams of CCD data into the array processor in an interleaved fashion. The data flow from the CCDs and the trigger signals drives the data acquisition cycle on an interrupt basis. There is no rigid sequencing built into the board to control the camera readout order or speed. It allows one, two, or three cameras, independently configured with different array readout sizes, to send pixels simultaneously and at different rates.

Each CCD camera presents a 9 bit pixel every $2 \mu \mathrm{s}$. The main interface board uses a 64 word deep, 15 bit word, first-in-first out (FIFO) buffer for each camera's data stream. Pixels accumulate in the FIFO buffers, which issue a half-full flag at 32 pixels. The time to half-full is 32 pixels $\times 2 \mu \mathrm{s} / \mathrm{pixel}=64 \mu \mathrm{s}$. The array processor can fetch a 16 bit word from the input port and write it to data memory in two $110 \mathrm{~ns}$ instructions. The pixels are burst-transferred to the array processor input port at its maximum rate in 32 pixels $\times 220 \mathrm{~ns} /$ pixel $=7 \mu \mathrm{s}$. The time to service all three half-full FIFO buffers, including an overhead of about $5 \mu$ s per 
camera, is $3 \times(7+5)=36 \mu \mathrm{s}$. This is smaller than the 32 pixel accumulation time so in principle the array processor can spend part of its time performing some background calculation during data acquisition.

Requests to the array processor, generated in hardware, are queued as conditions in a condition FIFO on the main interface board. The condition words are delivered to the array processor input port where they invoke appropriate software routines. Typical conditions are pixel FIFO half-full, camera done, TTL board input received, or error. In an elementary data acquisition cycle, for example, if the condition received were camera 1 pixel FIFO half-full, the array processor would select the camera 1 data channel, read and store 32 pixels, then reenable interrupts and wait for the next condition.

\subsubsection{Graphics Display Station}

The high resolution color display is a model One/75 made by Raster Technologies. It is interfaced to the $\mu$ VAX by a 16 bit, two-way DMA, DR11W board on the Q-bus. A $1024 \times 1024$ pixel image can be transferred to the screen in $\sim 4 \mathrm{~s}$. The One/75 has a $1280 \times 1024$ byte $\times 8$ bits deep video memory and a large $19^{\prime \prime}$ screen which can display the full $1280 \times 1024$ pixel image. An application can chorse up to 256 simultaneous colors by loading a look-up table which translates pixel intensity values into gray-levels or pseudocolors. The One/75 offers pan, up to 16 - 1 /old zoom, and a nondestructive cursor. These features are custrolled by an sitached data tablet. The cursor can be positioned on the tablet for readiback of coordinates and intensity values. The One/75 tablet and display screen also served as a measuring station for obtaining $(x, y)$ coordinate pairs along particle trajectories, the input for stereoscopic momentum reconstruction. A second device was obtained in fall 1989 when the measuring phase of the project was expanded.

The importance of visual feedback, throughout the hardware, data acquisition, and data reduction development phases of this project, cannot be overstated. Each step taken or software tool employed in the process of extracting charged particle kinematic information-from imaging through tracking, track measuring, track matching and momentum reconstruction-relied on visualization on the display screen for its confirmation.

\subsubsection{Data Acquisition Cycle}

The data acquisition cycle is interrupt driven. The program executing in the array processor first initializes the main interface board and the Multibus, initializes each CCD camera controller to the appropriate readout array size, then enables the first interrupt-accelerator beam spill on. While idle the CCDs are clocked to clear thermally generated charge. In response to the accelerator spill on signal from the TTL logic board, the code stops the continuous clear and opens the camera shutters. After receipt of the valid trigger signal, the cameras integrate for a programmable exposure time, the shutters are closed, and CCD readout is initiated. 
The data are buffered in the pixel FIFO's on the main interface board, which generate interrupts when they accumulate 32 pixels. The array processor services the interrupts and transfers the pixel data into memory. The end of the pixel data stream is signaled by a camera-done interrupt. After a maximum of $2 \mathrm{~s}$, the digitization and read out of all three CCD images is complete. The 9 bit CCD data words are then mapped into the intensity range 0-239 by a look-up table. The functional form of the mapping was a power law $f(x)=x^{\alpha}$, where $\alpha=\ln (239) / \ln (512)$. There were three reasons for the mapping from 9 to less than 8 bits. First, all data transfers in the computer system used 16 bit words, so by packing 2 pixels per 16 bit word, a factor of two in throughput was achieved. Second, 8 bit data words were a much more efficient format for buffering the large CCD images in the $\mu \mathrm{VAX}$ memory. Third, the 8 bit data words matched the depth of the display video memory, so the correspondence between the CCD data and its visual representation was 1-1 both in resolution and intensity. Sixteen levels of gray scale were reserved for overlays. After byte packing, the data are DMA transferred into free memory buffers in the $\mu \mathrm{VAX}$, where they are available for display, background processing and recording to tape. This sequence repeats once every accelerator spill cycle.

\subsection{Software}

Because of its novel design and because it was implemented virtually without antecedent, the Supervision System demanded a major software effort, first for system hardware development and debugging, then for data acquisition and control, and finally for data reduction and analysis. A number of different software environments evolved, for data acquisition, for the development of tracking and track matching algorithms, and for track measuring.

The major objectives of the data acquisition environment were to exploit as much as possible concurrent computing and I/O resources and to simultaneously provide the experimenter with a flexible, interactive environment for system control and for interactive, quantitative examination of the pictures. To achieve these goals, the software utilized multiple, asynchronous, concurrently executing computer tasks (VAX/VMS processes), deployed a large shared memory section for data and interprocess communication, and featured a flexible command stream that prioritized responding to real-time requests during standalone data acquisition.

Five concurrently executing processes comprised the data acquisition software. A user interface performed command parsing and distribution, called utilities for system configuration and debugging, and managed a set of screen windows that accepted terminal input, showed data buffer and process status, and offered online help. The other processes were viewed collectively as "background" processes. They were designed to logically separate control of the different physical devices and conceptually divide different system tasks. One background process was devoted to control of front end image acquisition from the CCD cameras and execu- 
tion of image processing operations on the array processor. A second was dedicated to displaying CCD images on the graphics station and managing input from the digitizing tablet. Embedded in the display process are a look-up table utility that, manipulates the translation of pixel intensity to pseudocolor representation and a profiling utility that uses cursor-driven readback of the video memory to plot intensity distributions and histogram intensity spectra. A third process was responsible for I/O operations between data buffers in the shared memory and disk or tape. The recording software evolved with the available technology until it could simultaneously and asynchronously drive four $8 \mathrm{~mm}$ tape devices and achieve a data rate of $4 \times 256 \mathrm{Kbyte} / \mathrm{s}=1 \mathrm{Mbyte} / \mathrm{s}$, sufficient to reach our design goal of writing one 3 Mbyte event per 6 s BEVALAC machine cycle. ${ }^{7}$ However, when front end I/O from the cameras and DMA transfers to the graphics display were contending for the same data path, the write rate in practice was bandwidth limited by the $Q$-bus to $~ 1.5$ Mbytes per BEVALAC cycle. The last background process extracted physics observables and chamber diagnostic information from the pictures, such as charged multiplicity per event $n_{c h}$ and average chamber intensity measures. From archive files of these data on disk, estimates of projected pseudorapidity distributions $d n / d \eta_{\text {proj }}$ and $n_{c h}$ vs $E_{T}$ correlations could be compiled.

The shared memory was implemented as a VAX/VMS global section. The global section was conceptually divided into a large data buffer for CCD images and a small reserved area for data structures for interprocess communication and buffer control. Access to the picture buffers is granted by distributed buffer management software which allocates pool space, coordinates data sharing, arbitrates access conflicts, and maintains process wait queues pending the availability of resources. Processes can request exclusive write access or shareable read access to the data buffers. The integrity of the control data structures and process synchronization is achieved by employing operating system event flags and resource locks. The exclusive allocation of memory pool space for storing picture data and the granting of shareable read access to existing pictures are completely dynamic. Background processes function asynchronously without explicit communication about the state of the memory buffers. During three-view data acquisition, six picture buffers were typically shared by the processes responsible for data recording, image display and physics calculation, while three buffers were being loaded with the next event.

\subsection{Optical Configurations and Resolution}

The Supervision System was used in two different modes in experiment NA35. During the first run, when the emphasis was on monitoring streamer chamber performance and the experimental trigger, the system was instrumented with only two cameras. During the second run, the system was extended with a third camera and used for data acquisition in addition to its supervisory capacity. Three stereo-

\footnotetext{
${ }^{7}$ Since the Supervision System was designed to image streamer chambers both at the CERN SPS and at the Berkeley BEVALAC, the shorter BEVALAC machine cycle placed the more stringent demands on the $\mathrm{I} / \mathrm{O}$ capability.
} 
scopic views were recorded for each event. The camera positions with respect to the magnetic bend plane are indicated in Figure 3.7. The cameras are nominally

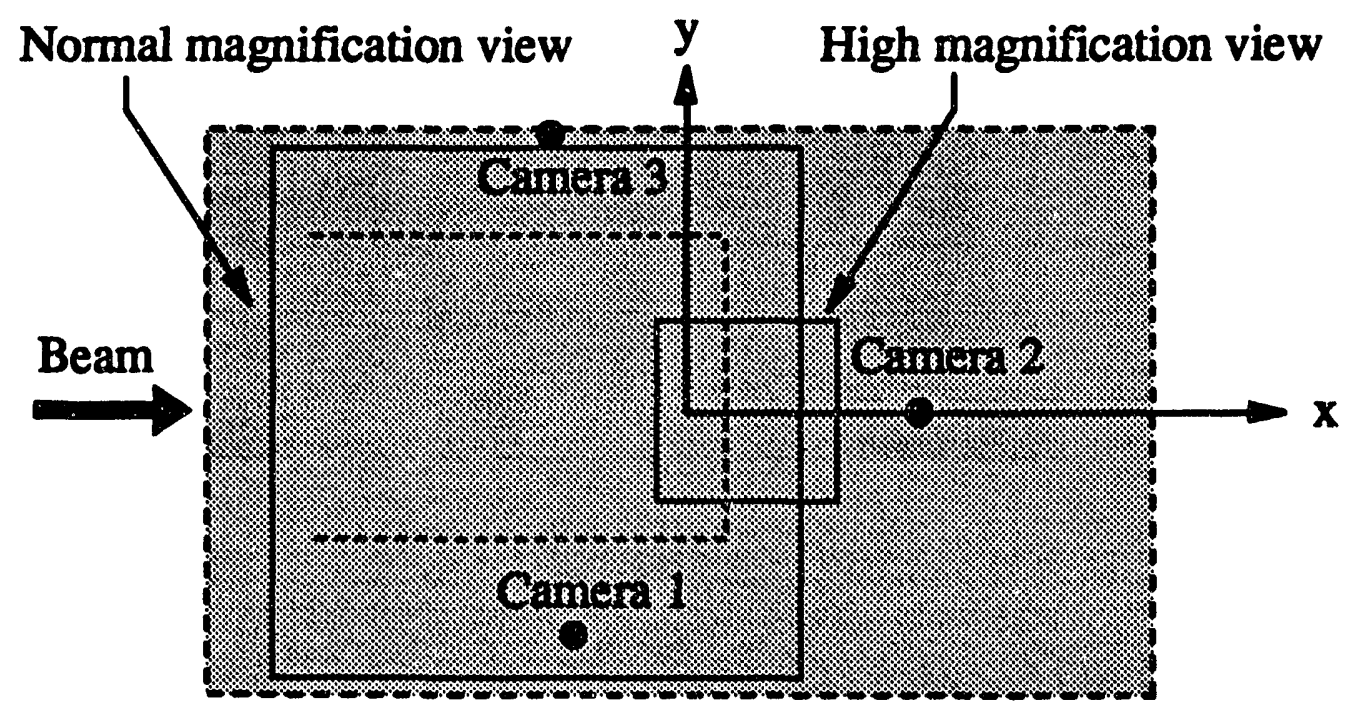

Figure 3.7: Camera positions. The shaded area is the streamer chamber sensitive volume. Also shown are the fields-of-view at the different magnifications. The dashed line that intersects both views is the contour employed for the online track finding.

located $\sim 330 \mathrm{~cm}$ above the midplane of the chamber. Cameras 1 and 2 imaged the chamber through the mirror housings. Camera 3 pointed directly into its camera port and was located $\sim 15 \mathrm{~cm}$ farther from the beamline than camera 1 . Space constraints imposed this asymmetry, which resulted in different stereo angles ${ }^{8}$ for the three different two-camera stereo systems. The cameras point approximately at the chamber midpoint-the axes of symmetry of the optical assemblies are only approximately perpendicular to the chamber midplane. The CCD image planes are inclined at small angles to the midplane.

The different lens configurations used in the different running modes are summarized in Table 3.2. In the two-camera monitoring mode, a $50 \mathrm{~mm}$ focal length primary lens was used on one camera and a $135 \mathrm{~mm}$ focal length lens on the other. The relay lens, a $50 \mathrm{~mm}$ focal length Macro, always imaged the output phosphor of the second stage intensifier onto the CCD at $M=1$. The normal magnification lens $M=65$ viewed $a \sim 1.2 \times 1.2 \mathrm{~m}^{2}$ square region of the chamber. The high magnification lens $M=23$ imaged a smaller $\sim .4 \times .4 \mathrm{~m}^{2}$ region at higher spatial resolution. These are indicated by the squares in Figure 3.7.

\footnotetext{
${ }^{8}$ The stereo angle between two cameras is defined from the position vectors from the chamber midpoint to the effective pinholes of each camera's optical system, $\cos \left(\theta_{a b}\right)=\vec{a} \cdot \vec{b} /(\|\vec{a}\|\|\vec{b}\|)$. The stereo axis of each camera pair is defined from the line segment connecting the pinholes. The role of the pinholes, determined by an optical constants program, and the significance of the stereo axis are discussed in Chapter 4.
} 


\begin{tabular}{||l|l||}
\hline \hline Primary lens (High magnification) & Pentax \\
\hline Focal length & $135 \mathrm{~mm}$ \\
\hline f-stop & $\mathrm{f} / 2.8$ \\
\hline Distance to midplane & $340 \mathrm{~cm}$ \\
\hline Demagnification at midplane & 23 \\
\hline Resolution (MTF $=50 \%)$ & $50 \mathrm{lp} / \mathrm{mm}$ \\
\hline
\end{tabular}

\begin{tabular}{||l|l||}
\hline Primary lens (Normal magnification) & Pentax \\
\hline Focal length & $50 \mathrm{~mm}$ \\
\hline f-stop & $\mathrm{f} / 2.8$ \\
\hline Distance to midplane & $330 \mathrm{~cm}$ \\
\hline Demagnification at midplane & 65 \\
\hline Resolution (MTF $=50 \%$ ) & $50 \mathrm{lp} / \mathrm{mm}$ \\
\hline
\end{tabular}

\begin{tabular}{||l|l||}
\hline Relay lens & Pentax \\
\hline Focal length & $50 \mathrm{~mm}$ Macro \\
\hline f-stop & $\mathrm{f} / 2.8$ \\
\hline Demagnification & 1 \\
\hline Resolution (MTF $=50 \%)$ & $50 \mathrm{lp} / \mathrm{mm}$ \\
\hline
\end{tabular}

\begin{tabular}{||l|l||}
\hline Stereo angle \\
\hline$\theta_{12}$ & $14.8^{\circ}$ \\
\hline$\theta_{23}$ & $17.2^{\circ}$ \\
\hline$\theta_{31}$ & $18.4^{\circ}$ \\
\hline \hline
\end{tabular}

Table 3.2: Characteristics of primary and relay lenses at high and normal magnification. 
Examples of each view from an ${ }^{16} \mathrm{O}+{ }^{197} \mathrm{Au}$ collision at $200 \mathrm{GeV} /$ nucleon, taken with the streamer chamber magnet off, are shown in Figures 3.8 and 3.9. Note the energetic $\delta$-rays that wander about the chamber. These curl up with the field on and appear as bright spots or give rise to flares. The two exceptionally bright tracks which approximately bound the central cone in Figure 3.8 are low momentum, target protons. The lower demagnification in Figure 3.9 enabled tracks in the central cone to be distinguished. The overlap of the different views can be identified from tracks which are imaged in both pictures, in this case from one of the bright protons, from the non-vertex track at slope $\simeq-1$, and from the seahorseshaped $\delta$-ray. Tracks found in the mutually exclusive regions of the different views can be combined to obtain the estimate oi $n_{c h}$. For the case of magnetic field off, the projected angle of emission and hence $\eta_{\text {proj }}$ can be calculated from the surveyed target position and a single track point. The approximate overlap of the different views and a three-sided, rectangular contour for online track finding are shown in Figure 3.7. For full three-view data acquisition, all cameras used the normal magnification primary lens. A representative CCD image taken in this configuration of a ${ }^{32} \mathrm{~S}+{ }^{32} \mathrm{~S}$ collision at $200 \mathrm{GeV} /$ nucleon with field on is shown in Figure 3.1.

An estimate of the resolution of an optical system ${ }^{9}$ can be obtained by calculating the effective source size of a luminous object, photographed and projected to full size in space, after folding in effects which degrade the resolution [Bul67]. According to Ref. Bul67, the effective source size is approximately the square root of the sum of the squares of the following terms:

1. Actual source size in space.

2. Diameters of the circles-of-confusion due to object depth-of-field, image intensifier photocathode and output phosphor nonflatness, and image depthof-field.

3. Diffraction from the lens apertures.

4. Resolutions of the lenses, intensifier assembly, and the CCD projected into space.

Explicit expressions for the different contributions can be found in Ref. Bul67. For the CCD camera optical configurations, the dominant terms are the real source size, the object depth-of-field, and the resolutions of the imaging devices:

$$
S_{e f f}^{2} \simeq s^{2}+\left(\frac{z-z_{0}}{\left(M_{1}+1\right) f / \#_{1}}\right)^{2}+\left(\frac{M_{1}}{R_{1}}\right)^{2}+\left(\frac{M_{1}}{R_{2}}\right)^{2}+\left(\frac{M_{1} M_{2}}{R_{3}}\right)^{2}+\left(\frac{M_{1} M_{2}}{R_{4}}\right)^{2} .
$$

$z-z_{0}$ is the distance from the chamber midplane and $f / \#_{1}$ is the $f$-number of the primary lens. $M_{1}$ and $M_{2}$ are the demagnifications of the primary and relay lens respectively. $R_{1}, R_{2}, R_{3}$, and $R_{4}$ are the resolutions in $1 \mathrm{p} / \mathrm{mm}$ of the primary

9J.W. Harris performed the initial optical resolution calculations. 


$$
\square
$$




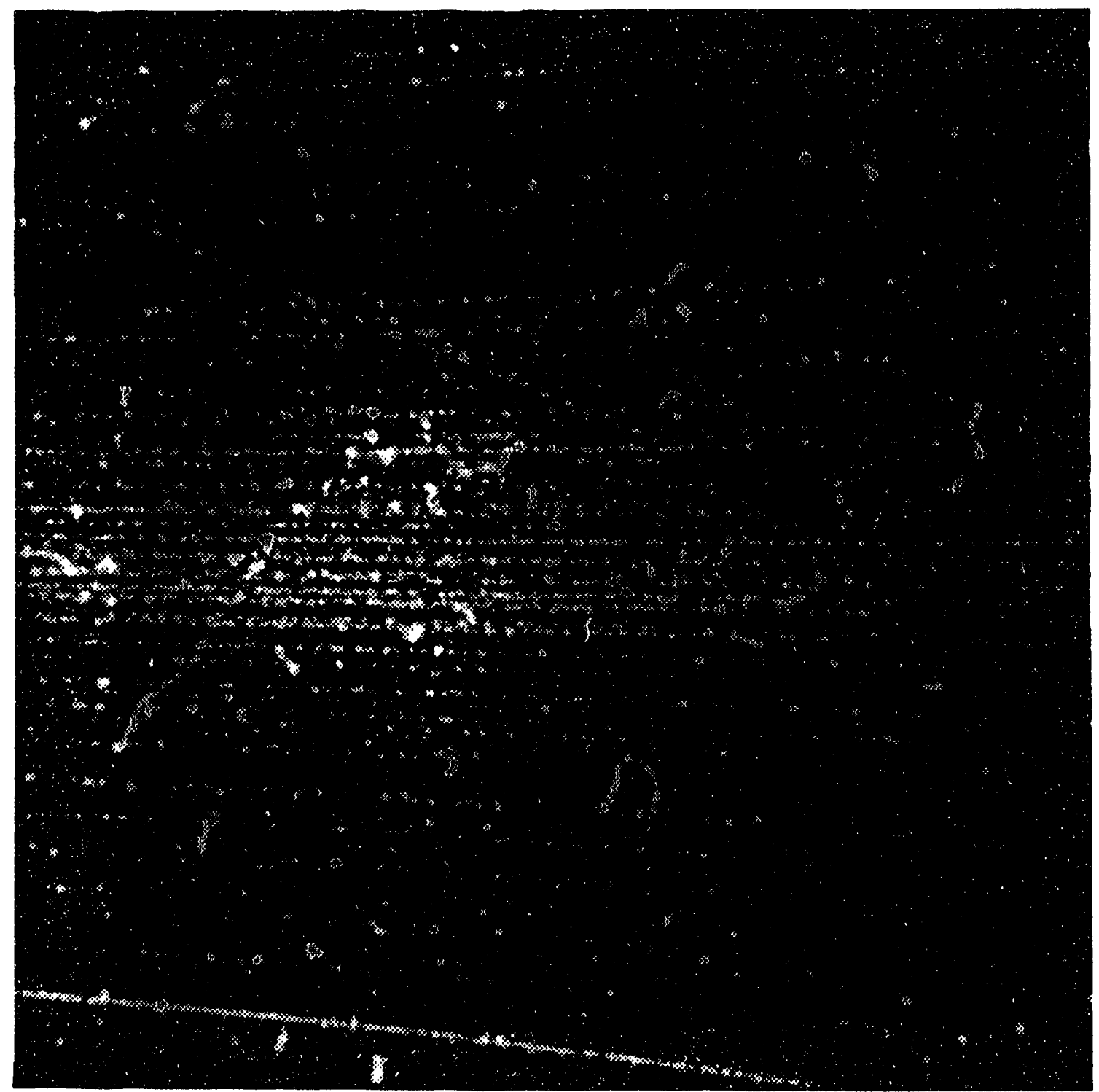

Figure 3.9: High magnification CCD image of the same event. The region of overlap can be identified by common features, such as the heavily ionizing proton near the bottom and characteristic $\delta$-rays, for example, the seahorse-shaped $\delta$-ray near the top left which intersects the downward sloping non-vertex track. 
lens, image intensifier assembly, relay lens and the CCD respectively. From the resolutions at 50\% MTF listed in Tables 3.1 and 3.2 the effective source size of a $1 \mathrm{~mm}$ diameter streamer at the chamber midplane should be $S_{\text {eff }} \simeq 8.0 \mathrm{~mm}$. The resolution is limited chiefly by the properties of the MCP intensifier. Employing the image intensifier limiting resolutions, $S_{\text {eff }} \simeq 3.7 \mathrm{~mm}$. Since $M_{2}=1$, at normal magnification a $1 \mathrm{~mm}$ diameter streamer would be imaged over a $\sim 5 \times 5$ pixel region on the CCD, which is what was observed. At high magnification, the effective source size at $50 \% \mathrm{MTF}$ and limiting resolution should be $\simeq \mathbf{3 . 0}$ and $1.6 \mathrm{~mm}$, respectively, leading to streamer images over somewhat larger pixel neighborhoods. A perfect optical instrument, limited only by diffraction and by the resolution of the CCD, would image a $1 \mathrm{~mm}$ diameter streamer over a $\sim 2 \times 2$ pixel region. The results of the complete calculation are shown in Figure 3.10, which

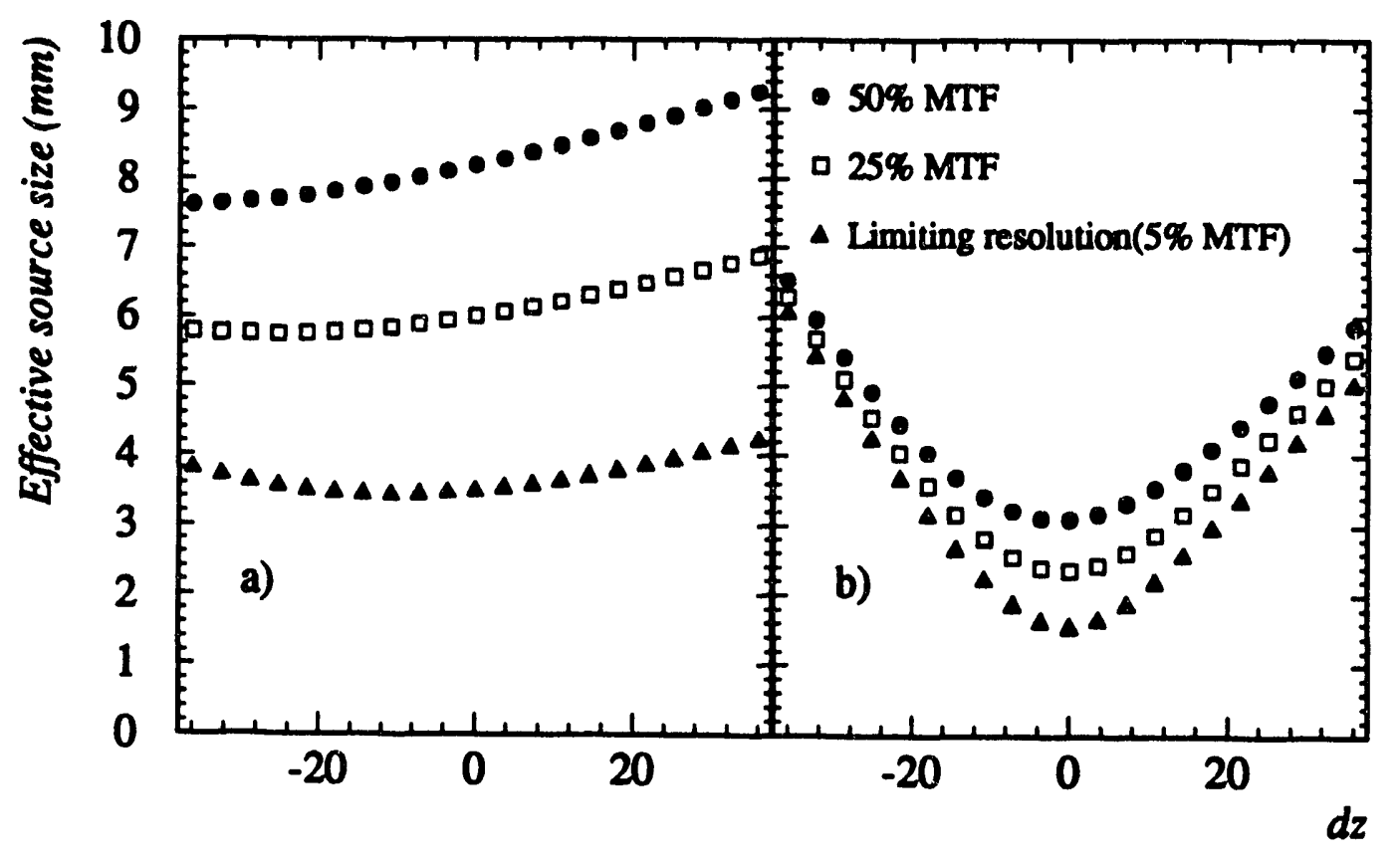

Figure 3.10: Effective source size of a $1 \mathrm{~mm}$ diameter streamer projected into the object plane as a function of $z-z_{0}$, the distance from the chamber midplane. The panels labeled a) and b) are for normal and high magnification, respectively. At normal magnification and 50\% MTF, the source size is dominated by the $M / R$ terms in Equation 3.1 which increase monotonically from the front to the back of the chamber. As the resolution goes up and/or the demagnification goes down the object depth-of-field contribution, which is approximately symmetric in $z-z_{0}$, introduces a quadratic modulation.

plots effective source size as a function of distance from the chamber midplane for both magnifications. Three different estimates of the lens and image intensifier resolutions were employed in the calculation, indicated in the figure by the change in MTF from $50 \%$ to $5 \%$ at limiting resolution. The CCD achieves $50 \%$ MTF at its limiting spatial frequency. At normal magnification, plotted in Figure 3.10a, and 
$50 \%$ MTF, the source size is dominated by the resolution terms in Equation 3.1. Since $R$ is fixed, these terms increase monotonically as $M=d / f-1$ increases from the front to the back of the chamber. The distance $d$ of a streamer from the effective pinhole ranges from $\sim 300-360 \mathrm{~cm}$. $f$ is the focal length of the primary lens. If $M / R \gg 1$, the object depth-of-field term, which is approximately symmetric in $z-z_{0}$, makes a negligible contribution when added in quadrature. As $R$ increases, the object depth-of-field introduces a quadratic modulation on top of a slowly varying pedestal. At smaller demagnification, plotted in Figure 3.10b, where the pedestal of minimum effective source size is lower, the quadratic modulation is more pronounced and is large enough in scale to be important at each resolution level.

It is generally assumed[Bul67] that a streamer position can be measured, either by visually estimating or by explicitly calculating the centroid of the streamer from the intensity distribution, with a precision which is a certain fraction of the effective source size. Assuming the center of a streamer can be located with an accuracy of $\pm S_{\text {eff }} / 10$, as is done in Bul67, the resolution at normal magnification and $50 \% \mathrm{MTF}$ is $1 \mathrm{~mm}$ in space. The large effective source size presents a problem, however, for the resolution of close track pairs. This leads to a reduction in detection efficiency near the central cone which we discuss in Chapter 4.

\subsection{Online}

The Supervision System was used in experiment NA35 to obtain immediate feedback on streamer chamber performance and the event trigger. It also provided a first look at the physics by analyzing events online and correlating streamer chamber and calorimetric observables. The online analysis and front end data acquisition of CCD images could be easily accomplished once every SPS machine cycle. For each CCD event trigger information from another computer was also recorded by means of the external terminal link shown in Figure 3.2. This information consisted of the corresponding roll and frame number for matching CCD, film and calorimeter data, and the energies deposited in the calorimeters.

CCD events were analyzed to determine track hits, from which the charged multiplicity $n_{\text {ch }}$ and the projected emission angle $\theta_{\text {proj }}$ could be calculated, and to evaluate several performance measures, which examined the average light intensity per streamer in various regions of the chamber. The online track finding was simple, defining track hits by applying a peak-finding algorithm to a one-dimensional intensity distribution sampled along a single pixel contour. The contour, indicated in Figure 3.7 by the interior dashed line, crossed both the normal and high magnification views.

The peak-finding algorithm is illustrated in Figure 3.11. The figure is a magnified view of a portion of the graphic display station screen. A segment of the single pixel contour in the normal magnification view is indicated by the green overlay. The green tick marks highlight the track-contour crossings found by the algorithm. The red histogram is the intensity distribution of the CCD image along the con- 


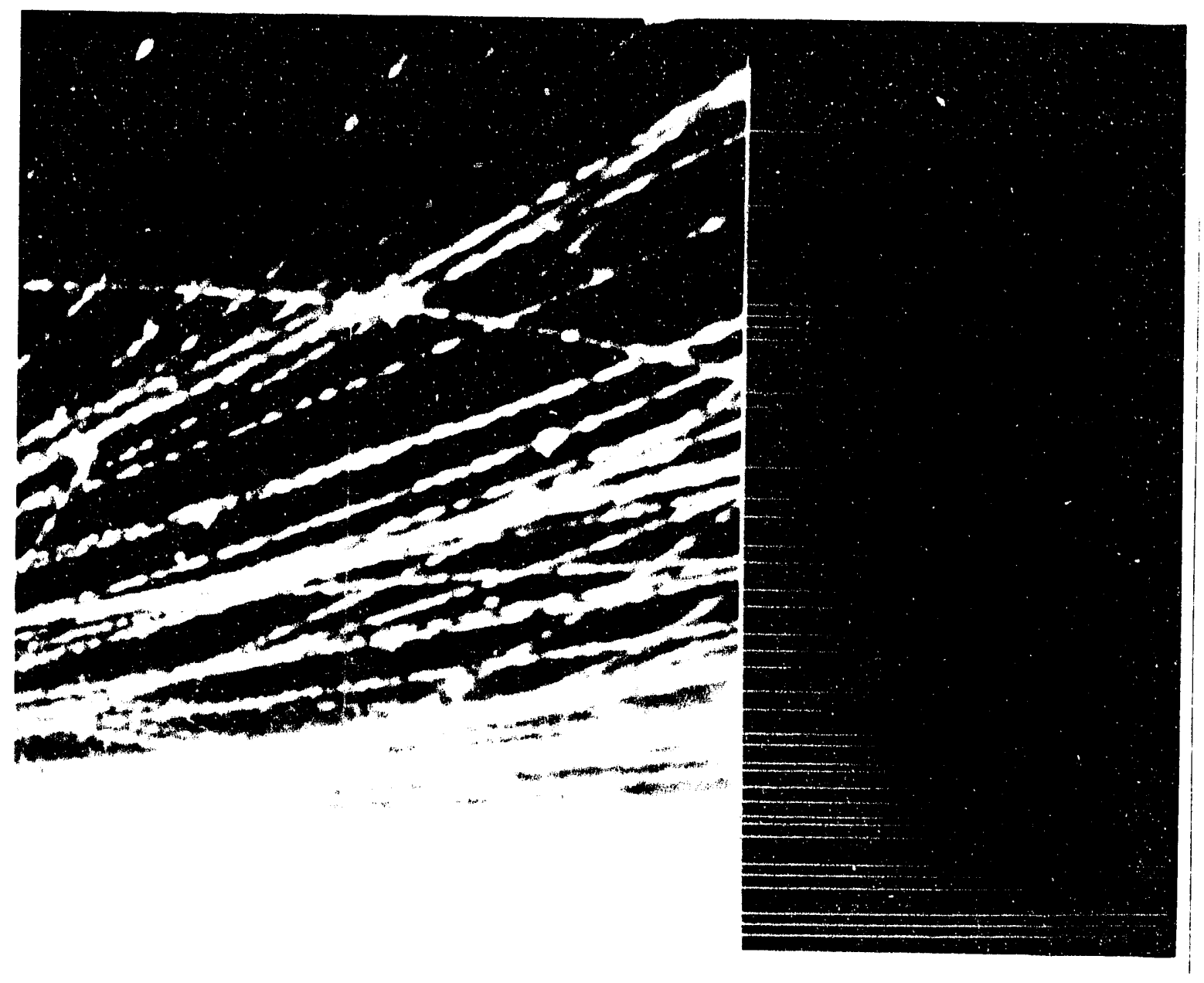

Figure 3.11: Peak-finding algorithm. The CCD intensity distribution along the one pixel wide green contour is displayed as the red histogram at the edge of the picture. 
tour, completely unprocessed except for the look-up table compression of the 9-bit $A D C$ digital number to a value between 0 and 239. The green overlays locate the peak positions found by the algorithm. Far from the cone, the signal is very clean with a small, camera-dependent background pedestal. Near the cone, the bright background introduces a higher pedestal, even for isolated tracks. The projected track density in the cone exceeds the resolution. In this region, track-finding is performed in the high magnification view.

Since the scale of streamer size is $\sim 3 \times 3$ to $7 \times 7$ pixels, the peak-finding algorithm looked for track structure over five pixel neighborhoods. A pixel $j$ was declared a candidate peak $P_{i}(j)$ if its intensity value $I(j)$ was a local maxima, if it exceeded the background threshold $t$, and if it exhibited sufficient sharpness $s$, defined with respect to its second-nearest neighbors:

$$
\begin{aligned}
I(j) & \geq I(j \pm 1), \\
I(j) & >t, \\
I(j) & >\frac{1}{s}\left(\frac{1}{2}\{I(j+2)+I(j-2)\}\right) .
\end{aligned}
$$

$s$ is a positive number less than 1 . For example, for a Gaussian intensity distribution with $\sigma=3$ pixels, $s=0.8$. Double counting of tracks which satisfied $I(j)=I(j \pm 1)$ and finding tracks more closely spaced than was consistent with the two-track resolution were suppressed by requiring at least three pixels between adjacent peaks:

$$
\left|P_{i}(j)-P_{i \pm 1}\left(j^{\prime}\right)\right| \geq 3 .
$$

The mutually exclusive contour segments from the two views were identified and $n_{c h}$ obtained by adding the tracks found in both views.

Trigger systematics and rough estimates of the target mass dependence of particle production could be quickly obtained at the $\sim 20 \%$ level from the event files written to disk by the online analysis. 


\section{Chapter 4}

\section{Track Measuring, Track Matching and Reconstruction}

\subsection{Introduction}

The streamer chamber is in many respects the successor to the bubble chamber. Its main advantages are its shori memory time, which can be tuned to permit operation in intense beams of $10^{6}$ particles per second [Eck77], and the ease with which it can be triggered. Only relatively recently have rapidly recycling, bubble chambers been developed which can be incorporated into hybrid spectrometers [Bal77] and triggered. ${ }^{1}$ The streamer chamber also benefits from its low mass which makes multiple scattering and energy loss negligible for most ionizing particles. One disadvantage is its poorer resolution. Judged by the intrinsic scale of their respective light sources, conventional modern streamer chambers [Aln87], [San87] achieve position resolutions of $\simeq 1 \mathrm{~mm}$ in space compared to the $\simeq 200 \mu \mathrm{m}$ resolution of the large modern bubble chambers [Fis73]. High pressure streamer chambers [Maj82], which are motivated by the importance of secondary vertex detection in searchs for short lived, heavy particle states, have obtained $50 \mu \mathrm{m}$ resolution. These efforts equal the precision of the latest holographic bubble chambers [Kit89] but have not as yet produced physics results. The streamer chamber shares with the bubble chamber the class of problems associated with stereoscopic track reconstruction, the task of determining three-dimensional space trajectories from two-dimensional projected images. Some of these problems are exacerbated by the high track multiplicities and extreme track densities encountered in high energy, nucleus-nucleus collisions where obscuration can undermine track measurement.

One inheritance from the bubble chamber is direct. The computer codes employed for stereoscopic momentum reconstruction of streamer chamber data are generally modifications of pre-existing bubble chamber codes. In particular, LBL and FNAL experiments have used the Three-View Geometry Program (TVGP)

\footnotetext{
${ }^{1}$ Trigger selection in a bubble chamber is for a given expansion cycle, not on a particle-byparticle basis and not on the $\mu \mathrm{sec}$ time scale. This limits the beam intensity to $\simeq 10^{3}$ particles per second.
} 
developed for the 72" bubble chamber [Sol65] while CERN experiments have used programs developed for the large chambers BEBC and Mirabelle [Lau72]. Because the bubble chamber was a seminal detector in the development of particle physics its capabilities have been extensively studied. In heavy liquids, multiple scattering and energy loss strongly perturb particles from their ideal trajectories. Refraction at the necessarily thick, optical windows significantly bends light rays from straight paths. These effects are virtually absent from streamer chambers. They are usually operated at atmospheric pressure where their sensitive gases, which present little material to energetic particles, can be contained by optically thin, transparent, mylar windows. Hence the reconstruction codes developed for bubble chambers are well equipped to handle the simpler versions of these problems when they arise in streamer chambers.

The principal objectives for the development of a CCD imaging system for the streamer chamber were to significantly accelerate the data reduction rate and to identify charged particles from an optical measurement of ionization. Visual tracking detector data from bubble chambers, streamer chambers and emulsions is conventionally hand measured by scanners. Because track matching, the association of different projected images with the same space curve, becomes much more difficult in a high multiplicity, high track density environment, the effort expended measuring $n$ tracks in a three-camera system increases much more rapidly than $3 n$. This is the motivation for computer matching. If the pattern recognition problem can be solved so that track measuring can be accomplished without scanner assistance, visual tracking detectors will be transformed into electronic ones, with a tremendous improvement in the statistical reach of the data.

In principle, both primary specific ionization and total specific ionization can be inferred from streamer chamber data, the former from streamer counting or gap length distributions, the latter from streamer brightness [Ric74]. Low momentum particles with $\beta<.7$ have been identified visually in this way for many years [Eck77]. Attempts to identify high momentum particles in the relativistic rise have also met with some success [Eck77], [Bas81], but much better $d E / d x$ resolution is required. Streamer brightness histograms for individual tracks imaged by the CCD Supervision System resemble the Landau energy loss distribution, indicating particle identification by $d E / d x$ might be possible especially in the $1 / \beta^{2}$ region of the energy loss curve. In spite of suggestive, preliminary correlations between brightness per unit length and momentum, a more thorough first analysis concluded that particle identification by $d E / d x$ was likely to fail, particularly at the high final-state momenta encountered at SPS energies. However, normalization corrections for event-by-event high voltage fluctuations and track-by-track corrections for trajectory-dependent effects, which were important for the MSU group ${ }^{2}$ [Kro90], were never applied. Since most charged particles with rapidity $1<y<3$ are near minimum-ionizing the potential phase space for $\pi, K, p$ separation by this method was small and this effort was abandoned. It may be revived for data taken at the BEVALAC, where most of the singly-charged particles from partic-

${ }^{2}$ The CCD system developed by MSU was employed for very different physics. See page 32. 
ipant matter have $p_{l a b}<1 \mathrm{GeV} / c$. Separate tests with higher resolution image intensifiers using cosmic rays [Roh89] indicated that adequate $d E / d x$ resolution could be achieved in the relativistic rise by deducing the primary ionization from avalanche counting.

Because of the square geometry of the CCDs, their limited resolution at appropriate demagnification, and our interest in particle identification by $d E / d x$, we elected to image a square region in the upstream part of the chamber. This is shown schematically in Figure 4.1. This choice of field-of-view maximized the spa-

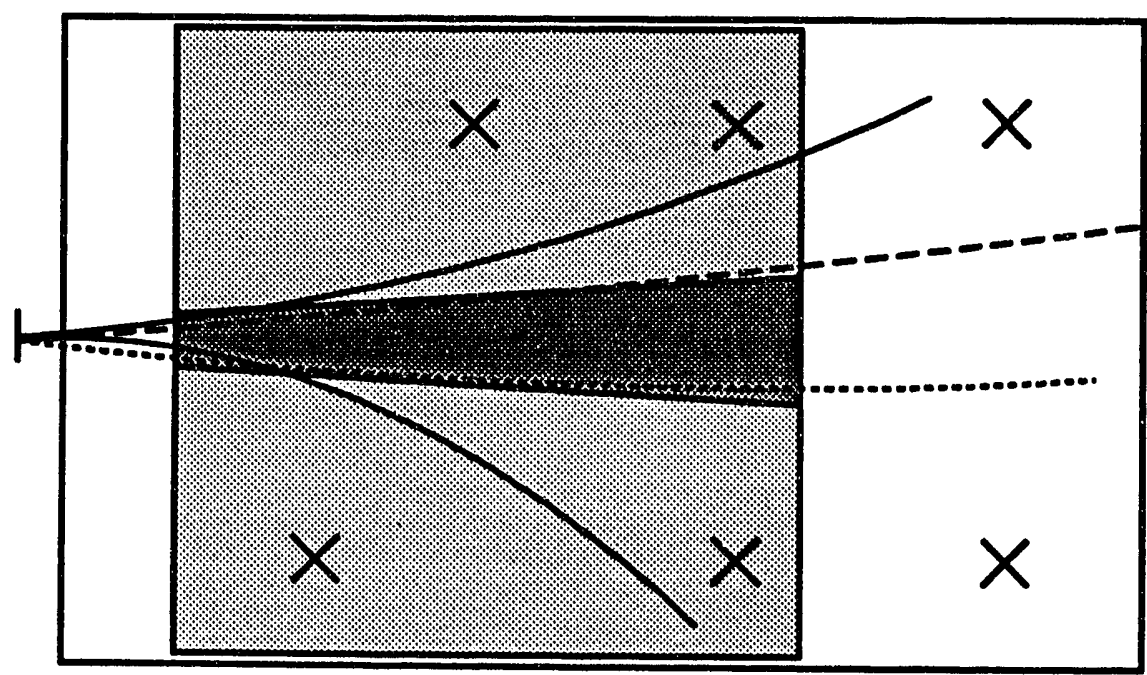

L

Figure 4.1: Square CCD image of a $\sim 1.2 \times 1.2 \mathrm{~m}^{2}$ section of the rectangular streamer chamber. The crosses are the fiducial marks. The darkened region at small laboratory angles is the central cone. The dashed tracks in the cone would be impossible to measure.

tial resolution, while achieving good phase space acceptance for charged particles in the laboratory momentum range we could conceivably identify by $d E / d x .^{3}$ The crosses included in the figure are the fiducial marks, reference points of known position required for momentum reconstruction. Also shown is the central cone, a region of extremely high track density at small laboratory emission angles. Tracks with projected images in this region, such as the double-dashed track in the figure, cannot be resolved or measured. The thick dashed track is at the edge of the CCD acceptance because of the short arc length available for measurement. It would be easily measured by the film cameras, which imaged the whole chamber onto $70 \mathrm{~mm}$ film. The presence of the cone leads to a dramatic reduction in detection efficiency near midrapidity.

The cone plays a dominant role in the analysis of our data. It diminishes the effective fiducial volume of the streamer chamber and defines the phase space

\footnotetext{
${ }^{3}$ Forward of $y=3$ charged particles are either minimum-ionizing and cannot be identified by $d E / d x$ or in the relativistic rise which requires much better $d E / d x$ resolution.
} 
acceptance of our measurement. Within that acceptance, the reduced detection efficiency near midrapidity leads to large correction factors and correspondingly large statistical errors. A number of different conecuts have been employed throughout the measurement and analysis of the data.

Traditionally, the problem of deducing space trajectories and momenta from multiple projected images has separated the two-dimensional track finding and measuring phase from track matching and stereoscopic track reconstruction. We have attacked the problem in this manner. A second approach divides point measurement, point matching and space point reconstruction from three-dimensional tracking and momentum estimation. This method has generally not been applied to conventional film-imaged data because of the dearth of quantitative information upon which the unambiguous association of measured points from different images can be made, and because of a more subtle problem associated with momentum fitting after the space trajectory has been determined-the residuals minimized during the momentum fit would be constructed from derived rather than measured quantities and the statistical distribution of the reconstructed space points about the nominal trajectory may be complicated and poorly known. This may undermine the statistical integrity of the fit[Soo67]. Taking advantage of the quantitative information made available by digitizing film images with a line-scanning CCD, this method has recently been implemented within the NA35 collaboration [Bun91], [Fuc91]. CCD-imaged data is obviously a natural candidate for application of the method.

\subsection{The Geometry of Photography and Reconstruction}

This section will describe the geometrical context in which visual tracking detectors like bubble chambers and streamer chambers make measurements and in which space trajectories are deduced from the two-dimensional projected images of photography. Several coordinate systems or spaces will be defined which will be referred to throughout this chapter and the remainder of this thesis. A number of additional concepts which are inherently geometrical in nature and commonly employed in the description of tracking detector data will be introduced. The general problem of helix fitting will be briefly discussed. The section will conclude with a discussion of the camera-to-camera mappings which transform the image of a space curve from one camera view into another. A track, the three-dimensional object characterizing a particle trajectory, should be distinguished from its twodimensional image. The term track will often be used when image is really intended but the meaning will be clear from the context.

Because of refractive effects and optical distortions in the imaging system, and because of the use of different display and digitization schemes for the measurement of photographic images, three coordinate systems must be employed:

1. The chamber space. 
2. The measurement space.

3. A reference system, often referred to in bubble chamber literature as the ideal film plane system.

These are depicted in Figure 4.2. Chamber space is the three-dimensional sensitive volume of the streamer chamber, a subset of the laboratory coordinate system. The convention for defining the $x, y$, and $z$ axes is indicated in the figure. The beam enters from the left and points in the positive $x$ direction. The origin of the coordinate system is chosen at the midpoint of the chamber. Most of the data taken by experiment NA35 used an external target upstream of the chamber entrance window at $(x, y, z) \simeq(-108,0,0)$. The magnetic field $\vec{B}$ points in the negative $z$ direction with the axis of symmetry of the superconducting dipole passing through the origin at $x=y=0$. Since $\vec{B}$ is parallel to $z$, the $x y$ plane is the bend plane. The midplane of the magnet coincides with the midplane of the chamber.

Measurement space is the two-dimensional plane surface in which sets of $\left(x^{\prime \prime}, y^{\prime \prime}\right)$ points are assigned to track images. Because measurement space is nearly parallel to the bend plane, the projected trajectory of a charged particle is well approximated by a circle. The process of photography may be viewed abstractly as a nonlinear mapping $M: R^{3} \rightarrow R^{2}$ from chamber space to measurement space, indicated in the figure by the arrow mapping the point $\left(x_{c}, y_{c}, z_{c}\right) \rightarrow\left(x_{m}, y_{m}\right)$. This is not strictly correct because characteristic features of the display and digitization system are interposed between the $\operatorname{CCD}($ film $)$ image and the measurement space. For a film measurement, for example, the demagnification of the optical system which projects the image onto the scanning table and the resolution of the digitizer critically influence the measurement. The coordinates in measurement space are arbitrary and their natural unit is the pixel, a dimensionless quantity corresponding to the "least count" of the digitization device.

Because the measurement space has no well-defined, physical relationship to the chamber space and because of optical distortions, it is unsuitable for track reconstruction and the reference system is introduced. Definition of the film plane system relies on an idealized representation of a camera consisting of a pinhole, an optical axis and an image plane, the ideal film plane. The pinhole is specified by the position vector $\vec{c}$ from the origin to the lens of the equivalent pinhole camera. The optical axis is somewhat arbitrary and does not necessarily coincide with the physical symmetry axis of the real optical imaging device. It is defined to be the line connecting the origin of coordinates in chamber space to the pinhole. The optical axis vector $\vec{a}$ points from the origin towards the pinhole. An arbitrary plane perpendicular to the optical axis can be designated as the film plane. The distance $f$ of the film plane from the pinhole is generally chosen so that $\|\vec{c}\| / f$ equals the camera demagnification. The film plane system is the three-dimensional coordinate system, indicated by the dashed frame in Figure 4.2 , whose $x y$ plane coincides with the film plane and whose $z$ axis points along the optical axis vector. For example, the coordinates of the camera pinhole in the film plane system are $\left(x^{\prime}, y^{\prime}, z^{\prime}\right)=(0,0,-f)$. Given $\vec{c}$ and $\vec{a}$ the orthogonal transformation from chamber space to the film plane system can be calculated in a straightforward manner. A 


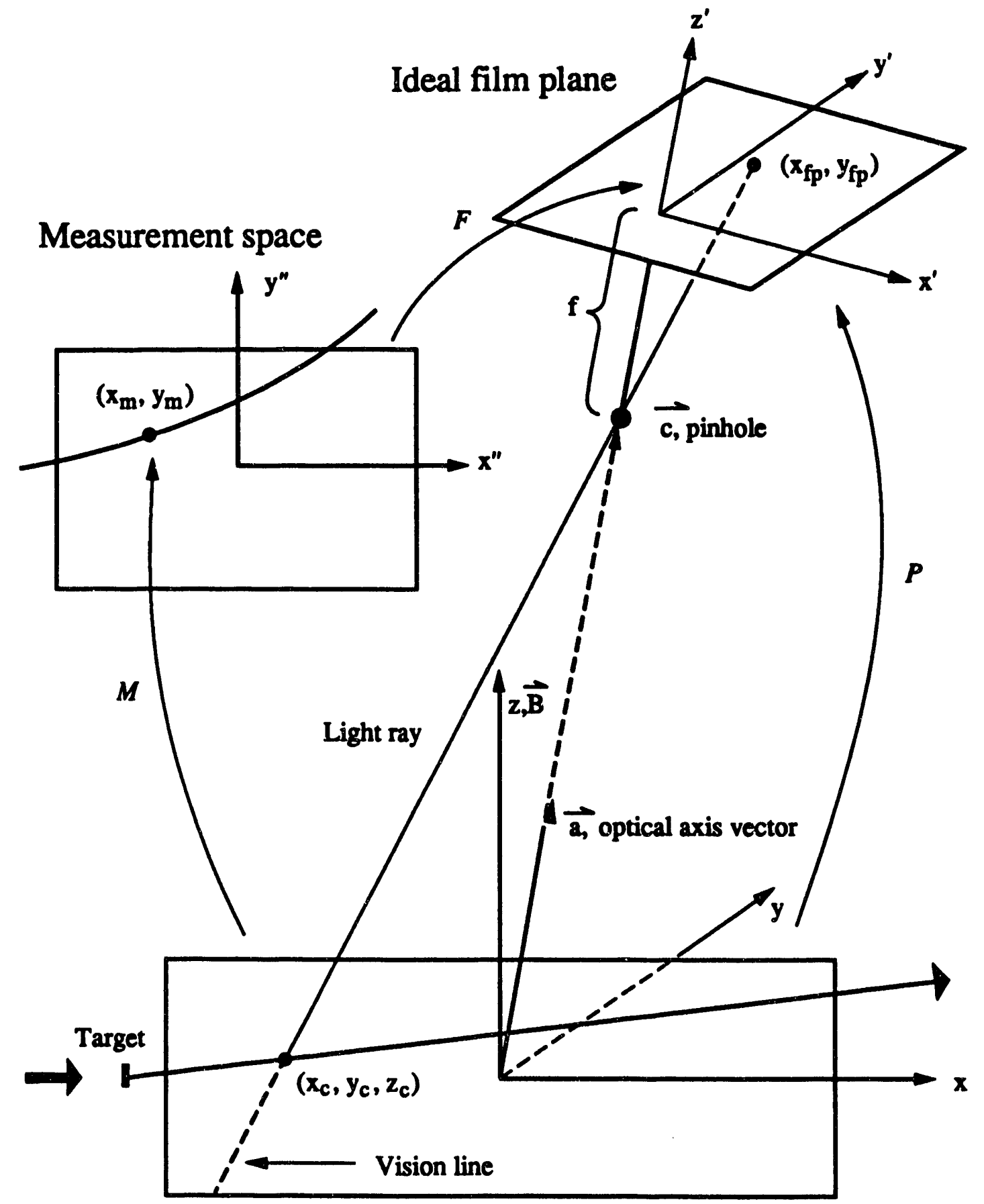

L

\section{Chamber space}

Figure 4.2: The three spaces employed in the measurement and reconstruction of streamer chamber images. The figure illustrates the non-bend plane in chamber space, the bend plane trajectory in measurement space and the ideal film plane. The mappings $M, P$, and $F$ are indicated by arrows. 
different choice for the reference system, motivated by the strong refractive effects in bubble chambers and relying on the concept of apparent position, is described in Ref. Soo67.

Points in the chamber space have a precise relationship to points in the film plane-they project along light rays which pass through the pinhole. This can be represented by a linear, projective mapping $P: R^{3} \rightarrow R^{2}$ from chamber space to film plane. Both the light ray and the mapping $P$ are indicated in Figure 4.2, the light ray passing from $\left(x_{c}, y_{x}, z_{c}\right) \rightarrow\left(x_{f p}, y_{f_{p}}\right)$ and the arrow representing the mapping $P$. Since $P$ is a projective mapping it is non-invertible. If the light ray is extended to its boundaries in chamber space it traces out a vision line : any space point lying on a vision line maps to the same image in the film plane under $P$. When image points in the reference systems associated with two different cameras are known to derive from the same space point the images are referred to as corresponding points. A space point can be reconstructed from two or more corresponding points by calculating the intersection of the vision lines.

The fiducial transformation from the measurement space to the film plane is most generally a six-parameter mapping $F: R^{2} \rightarrow R^{2}$, consisting of a translation plus a non-orthogonal matrix which permits different scale factors in the different directions [Soo67]:

$$
\left(\begin{array}{l}
x_{f_{p}} \\
y_{f_{p}}
\end{array}\right)=\left(\begin{array}{l}
f_{1} \\
f_{2}
\end{array}\right)+\left(\begin{array}{ll}
f_{3} & f_{1} \\
f_{5} & f_{6}
\end{array}\right)\left(\begin{array}{l}
x_{m} \\
y_{m}
\end{array}\right) .
$$

If the optical system were free of distortion $P=F \circ M$. The circle means the composition of two mappings, $M$ followed by $F$. $F$ is called a fiducial transformation because it can be deduced from fiducial marks, fixed points in the chamber space, by comparing their images under $P$ to their images under $M$. The role of optical constants programs is to fix the position of the pinhole, calculate the mapping $F$ and parametrize the optical distortion from the difference between $P$ and $F \circ M$. Once measurements are transformed to the film plane and distortion-corrected, geometrical reconstruction can begin.

The trajectory of a charged particle in a homogeneous magnetic field in the absence of physical perturbations like energy loss and multiple scattering is a helix. The equation of the helix in the chamber space [Raj75] can be expressed as a function of a parameter $\theta$ :

$$
\begin{aligned}
& x=\alpha+r \cos \theta \\
& y=\beta+r \sin \theta \\
& \dot{z}=z_{0}+(k / 2 \pi) \theta .
\end{aligned}
$$

$(\alpha, \beta)$ and $r$ specify the axis of symmetry and radius of the cylindrical surface on which the helix resides. The pitch $k$ represents the change in $z$ after one complete revolution in the $x y$ plane, the bend plane. $z_{0}$ is the value of $z$ when the parameter $\theta=0$. The motion in the non-bend planes is sinusoidal. If the distance $l$ traversed by a charged particle in the bend plane is small such that $l / r \ll 1$, the out-of-plane trajectory is well approximated by a straight line. 
Because of energy loss and inhomogeneities in the magnetic field, the trajectory of a charged particle with a given mass becomes more complicated but still can be put into a standard parametrization ${ }^{4}$ [Lau72] determined by the following set of six parameters:

1. $x_{0}, y_{0}, z_{0}$ : the coordinates of a reference point on the track. They usually have the meaning of the start point of the track.

2. $\lambda_{0}, \phi_{0}$ : two angles, the dip and azimuth, which specify the direction of motion at the start point.

3. $1 / p_{0}: p_{0}$ is the momentum of the charged particle at the start point.

The standard parametrization is illustrated in Figure 4.3. The start point is indi-

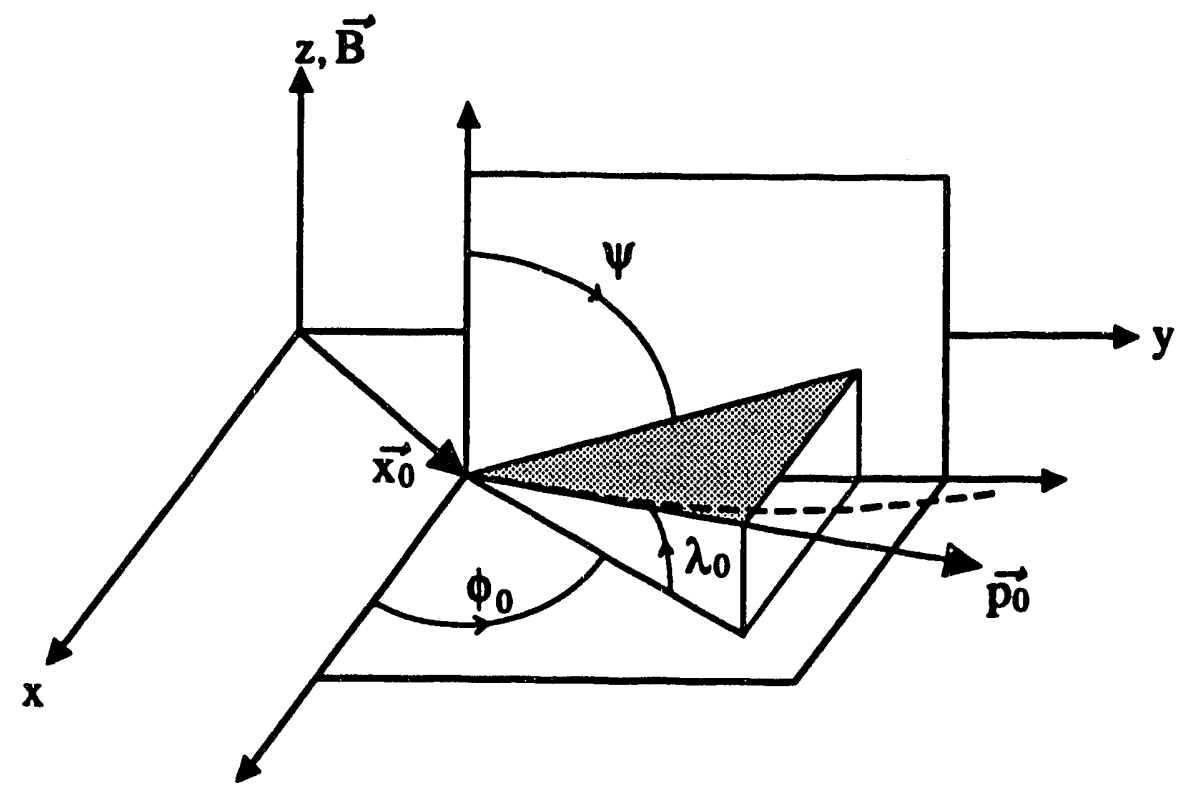

\section{L}

Figure 4.3: Standard parametrization of a charged particle trajectory. The start point is indicated by the vector $\overrightarrow{x_{0}} . \psi$ is the azimuthal angle in the transverse momentum plane, measured from the $z$ axis.

cated by the vector $\overrightarrow{x_{0}}$. The correspondence between the helix parameters $r$ and $k$ and the parameters $p_{0}$ and $\lambda_{0}$ is direct: $\tan \lambda_{0}=k /(2 \pi r), p_{0} \cos \lambda_{0}=r B_{z}$. Since the reference point is arbitrary, one coordinate is usually fixed. The goal of track fitting is to deduce the remaining five parameters.

Also shown in Figure 4.3 is the azimuthal angle in the transverse momentum plane $\psi$. The polar coordinate angles with respect to the beam direction $x$ are

${ }^{4}$ The NA35 reconstruction program, based on the HYDRA data management system developed at CERN, is a literal implementation of the method described in Ref. Lau72. The discussion of helix fitting above is drawn from this paper. 
$(\theta, \psi)$. Our convention is to measure $\psi$ from the $z$ axis towards the $y$ axis. Positively charged particles with $0<\psi<\pi$ have azimuth $0<\phi<\pi / 2$ and are referred to as rightside particles: ${ }^{5}$ the magnetic field bends them away from the beam without crossing it. Conversely, positive particles with $\pi<\psi<2 \pi$ have $3 \pi / 2<\phi<2 \pi$ and are called wrongside: the magnetic field bends them back towards the beam. These definitions and angular domains are illustrated in Figure 4.4 which shows the bend plane trajectories and the projections of the momentum vector $\vec{p}$ into
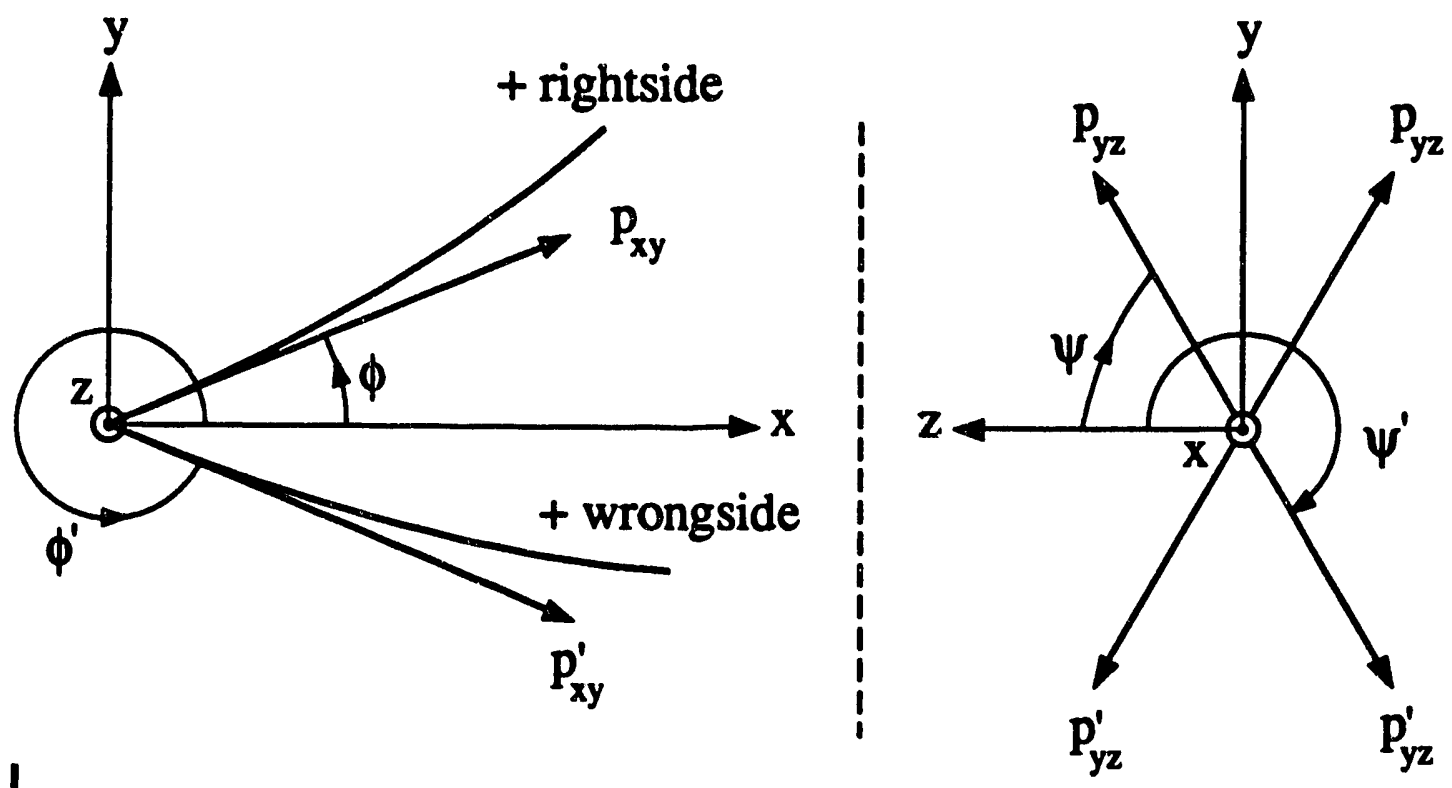

Figure 4.4: Kinematic relations between rightside and wrongside tracks. $p_{x y}$ and $p_{y z}$ are the projections of $\vec{p}$ into the bend and transverse momentum planes.

the $x y$ (bend) and $y z$ (transverse momentum) planes. Four tracks are illustrated, all of which have the same $\|\vec{p}\|$ and the same polar angle $\theta$. The kinematic variables of the wrongside tracks are identified with primes. Both members of the pairs of rightside and wrongside tracks have identical bend-plane trajectories, the same $p_{x y}=\|\vec{p}\| \cos (\lambda)$, and the same $\phi$, they differ only by the sign of the dip $\lambda$. Their $\psi$ angles are mirror symmetric about the $x y$ plane, $\psi \rightarrow \pi-\psi$ and $\psi \rightarrow 2 \pi-\psi$, respectively. Rightside and wrongside tracks with the same $\lambda$ and $\theta$ have azimuths which satisfy $\phi^{\prime}=2 \pi-\phi$. Analogous definitions hold for negatively charged particles. Because of the large Lorentz boost imparted to most particles in a fixed-target experiment at $200 \mathrm{GeV} / c$ many wrongside tracks with near-beam $\phi$ angles appear as rightside particles. The rightsides for positively and negatively charged particles, respectively, are loosely referred to as the positive and negative sides of the chamber. Because of obscuration caused by the beam pencil, the

\footnotetext{
${ }^{5}$ This is true because the streamer chamber running with an external target has virtually no acceptance for backward going particles.
} 
streamer chamber acceptance for given $(p, \theta)$ is dominated by the asymmetry of rightside and wrongside charged particles with respect to the beam.

In the standard parametrization the trajectory of a particle is a function of the arc length $s$ :

$$
\begin{aligned}
& x=x_{0}+\int_{0}^{e} \cos \phi \cos \lambda d s \\
& y=y_{0}+\int_{0}^{e} \sin \phi \cos \lambda d s \\
& z=z_{0}+\int_{0}^{e} \sin \lambda d s .
\end{aligned}
$$

Here $\lambda$ and $\phi$ give the instantaneous direction of motion in space. They are functions of the momentum $p(s)$ and the magnetic field $\vec{B}(s)$ as well as the arc length:

$$
\begin{aligned}
& \phi=\phi_{0}+\int_{0}^{s} \frac{(e / c) B_{\phi}}{p} d s \\
& \lambda=\lambda_{0}+\int_{0}^{s} \frac{(e / c) B_{\lambda}}{p} d s .
\end{aligned}
$$

$B_{\lambda}$ and $B_{\phi}$ are the components of $\vec{B}$ which account for local changes in $\lambda$ and $\phi$. $p$ can be determined from $p_{0}$ by the range-momentum relation. These equations can be integrated numerically to give the coordinates $(x, y, z)$ of any point on the track. The standard parametrization reduces to the helix representation if energy loss can be neglected so that $p=p_{0}$ and if $\vec{B}=B \hat{z}$ is constant.

Track fitting consists of making corrections to an initial estimate of the track parameters by minimizing the perpendicular distances from the track to the measured points in the film plane. The deviations $d_{i}$ are functions of the parameters and the measurements,

$$
d_{i}=f\left(x_{i}, y_{i} ; \lambda_{0}, \phi_{0}, 1 / p_{0}, x_{0}, y_{0}\right) .
$$

This expression is linearized to yield

$$
d=d_{0}+\frac{\partial f}{\partial \lambda_{0}} \delta \lambda_{0}+\frac{\partial f}{\partial \phi_{0}} \delta \phi_{0}+\frac{\partial f}{\partial\left(1 / p_{0}\right)} \delta\left(1 / p_{0}\right)+\frac{\partial f}{\partial x_{0}} \delta x_{0}+\frac{\partial f}{\partial y_{0}} \delta y_{0} .
$$

The partials here are functions of $\partial x / \partial \zeta, \partial y / \partial \zeta, \partial z / \partial \zeta$, where $\zeta$ may be any of the track parameters. They can be expressed analytically by differentiating Equations 4.3 and 4.4 and evaluated by numerical integration. The corrections to the estimated track parameters are obtained from an iterative least-squares fit which minimizes

$$
\chi_{f i t}^{2}=\sum_{i=1}^{n} \frac{d_{i}^{2}}{\sigma_{i}^{2}} .
$$

The error on each measurement $\sigma_{i}$ is taken to be a constant, the setting error.

The track fit is performed in a local frame $\left(x^{\prime}, y^{\prime}, z^{\prime}\right)$ obtained by a translation to the start point and a rotation such that $\vec{p}_{0}$ lies along the $y^{\prime}$ axis and $\lambda^{\prime}=0$. Five 
parameters are varied independently in the primed system: the radius of curvature, the azimuth, the dip out of the $x^{\prime} y^{\prime}$ plane, and the two start point coordinates in the $x^{\prime} y^{\prime}$ plane. The track fit system is updated with each iteration until the fit converges. After convergence the final parameters are transformed back to the chamber space. In spite of the fact that at each iteration step, only two start point coordinates are varied, $x_{0}, y_{0}$, and $z_{0}$ may all have changed during the fit.

In order to calculate the deviations in the film plane, each measured point must be associated with a space point on the track and a local track tangent. This is done by integrating the equations of motion Equations 4.3 and 4.4 up to an approximate arc length $s$ from the approximate track parameters. An estimate of the vertex position provides the approximate values for $x_{0}, y_{0}$ and $z_{0}$. The estimates of the track parameters $p_{0}, \lambda_{0}$ and $\phi_{0}$ and the arc lengths $s_{i}$ are furnished by performing an approximate fit based on optics and geometry. With the exception of a small class of measured points associated with characteristic physical effects, for example secondary vertices, kinks, ${ }^{6}$ energetic $\delta$-rays or exceptionally bright streamers, corresponding points cannot be found. The approximate fit is based on non-corresponding point (NCP) reconstruction.

NCP reconstruction depends on the construction of a space surface from two vision lines in one camera view, denoted the auxiliary view, which approximately contains the track. If a measurement in a different camera view, denoted the primary view, is intermediate in arc length between the measurements in the auxiliary view used to construct the approximating surface, a space point can be found from the intersection of the vision line with this surface. The two camera views form a stereo system. If track images have been measured in three views, three stereo systems can be employed; if only two track images have been measured only one stereo system is available for NCP reconstruction.

The two-dimensional vector $\vec{s}$ connecting the camera pinholes of a stereo system

$$
\vec{s}=\left(x_{c_{2}}-x_{c_{1}}, y_{c_{2}}-y_{c_{1}}\right)
$$

is called the stereo axis [Raj75]. If $\vec{t}$ is the track tangent in the auxiliary view it is shown in Ref. Soo67 that the uncertainty in $z$, in track depth in the chamber, is

$$
d z \propto 1 /\{\hat{z} \cdot(\vec{s} \times \vec{t})\}
$$

The uncertainty $d z$ is inversely proportional to the sine of the angle between the track and the stereo axis. This can be understood intuitively. If a track lies entirely in the plane spanned by the stereo axis and any vision line in the auxiliary view, a vision line in the primary view lies in the same plane, that is, $\vec{s} \times \vec{t}=0$ and the intersection point is completely undetermined. If the uncertainty is so great that the NCP reconstruction is unreliable, a track is said to possess bad stereo with respect to the given stereo system. In principle only two views are required to reconstruct a space curve. With three views the space points may be more precisely calculated by weighted averaging of the results from all acceptable stereo

\footnotetext{
${ }^{6}$ Kinks are points at which the curvature of a track has an apparent discontinuity. They result from a charged decay with a neutral daughter, for example $K^{+} \rightarrow \pi^{+} \pi^{0}$ or $\pi^{+} \rightarrow \mu^{+} \nu$.
} 
pairs. Bad stereo leads to a significant difference in reconstruction efficiency for two-view as compared to three-view reconstructions because only one stereo system rather than three is available for constructing the NCPs .

Attempts to accelerate the measurement of visual tracking detector data rely on predictive techniques which reduce measuring effort by using the measurements in one view to restrict the search space in a second. The principle underlying these methods is illustrated in Figure 4.5. Each measurement $\left(x_{1}^{\prime}, y_{1}^{\prime}\right)$ in the film plane of camera 1 is associated with the set of points in the chamber space $\left(x_{c}, y_{c}, z_{c}\right)$ comprising its vision line. This set is mapped under $P_{2}$, the projective mapping for camera 2, to a two-dimensional curve $\gamma$ in the film plane of camera 2. $\gamma$ is bounded by the images under $P_{2}$ of the vision line-chamber boundary intersections. If a measurement is known to correspond to a chamber exit point, $\gamma$ reduces to the two boundary points. In Ref. Raj75 it is explicitly shown that for the case of a stereo system with both film planes perpendicular to the $z$ axis and with $z_{c_{1}}=z_{c_{2}}$, the equation for the curve $\gamma$ is

$$
\begin{aligned}
& x_{2}^{\prime}=x_{1}^{\prime}+\left(1-\frac{f}{z_{c_{2}}-z}\right)\left(x_{c_{2}}-x_{c_{1}}\right) \\
& y_{2}^{\prime}=y_{1}^{\prime}+\left(1-\frac{f}{z_{c_{2}}-z}\right)\left(y_{c_{2}}-y_{c_{1}}\right) .
\end{aligned}
$$

In Equation 4.10 the coordinate $z$ takes on all values in the chamber space, i.e., $z=[+36,-36]$. The camera-to-camera mapping $C: R^{2} \rightarrow R^{2}$ from the film plane of one camera to the film plane of another is a line segment parallel to the stereo axis. For the NA35 CCD cameras, the angles between the optical axes and the $z$ direction are $\simeq 10^{\circ}$ and $z_{c_{1}} \simeq z_{c_{2}} \simeq z_{c_{3}}$, approximately satisfying the assumptions of Equation 4.10.

A second construction $C^{\prime}$ which maps points from film plane to film plane is also illustrated in the figure. From the last imaged point in a given view, two planes are defined from the vision line-chamber boundary intersections. Each plane is spanned by a vector drawn from the target to the intersection point and by $\hat{y}$ the unit vector along the $y$ axis. Because the out-of-plane trajectory is sinusoidal the space curve associated with this track image must pass through the subset of chamber space bounded by the two planes. These planes may be denoted maximal dip planes because highly dipping tracks, which exit the chamber at the top or bottom at $z= \pm 36 \mathrm{~cm}$, would travel in them if the linear approximation to the out-of-plane trajectory were valid. Instead of the vision line-chamber intersections, the domain for $P_{2}$ is restricted to points bounded by the vision line-dip plane intersections. Like $C$, the camera-to-camera mapping $C^{\prime}: R^{2} \rightarrow R^{2}$ is generally a point-to-line transformation. The range of $C^{\prime}$ is the curve $\gamma^{\prime}$, a subset of $\gamma$. Both $\gamma$ and $\gamma^{\prime}$ are shown in Figure 4.5. By construction, the image of the target in camera 1 maps to a point in camera 2 under $C^{\prime}$. The set of mapped line segments which constitute the image of a track under $C^{\prime}$ focuses to a point. In the optical consistency calculation described in Section 4.6.1, both $C$ and $C^{\prime}$ are extended to the measurement space by composition with the inverses of the fiducial transformation $F$ defined in Equation 4.1 and the distortion correction mapping 


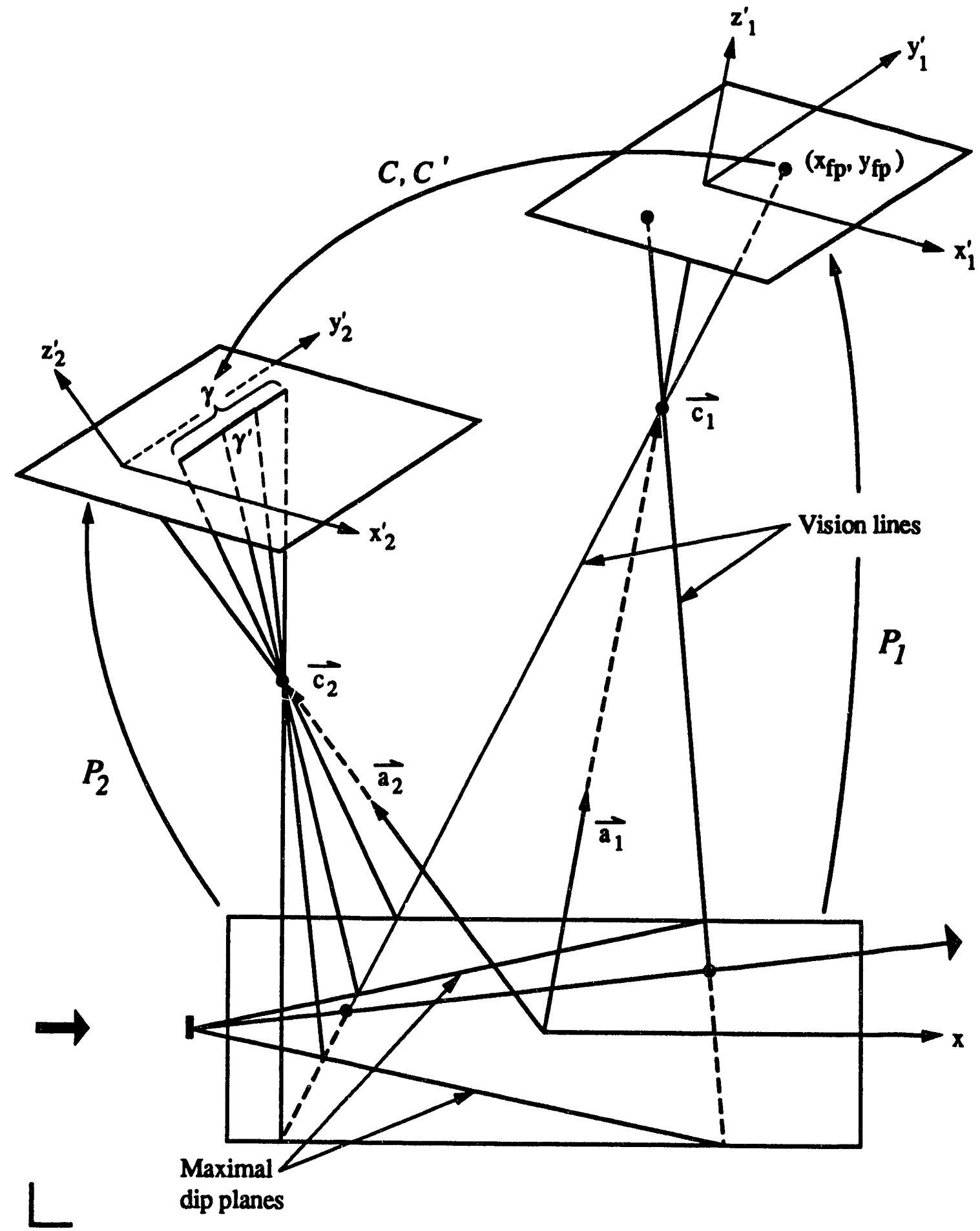

Figure 4.5: Camera-to-camera mappings $C$ and $C^{\prime}$. The images of the point $\left(x_{f p}, y_{f_{p}}\right)$ under $C$ and $C^{\prime}$ are the curves $\gamma$ and $\gamma^{\prime}$, respectively. The maximal dip planes are constructed from the vision line-chamber boundary intersections of the last measured point. 
$D C:$

$$
C_{\text {extended }}=F^{-1} \circ D C^{-1} \circ C .
$$

This expression means $C$ followed by $D C^{-1}$ followed by $F^{-1}$. The distortion correction mapping is described in $\hat{t}^{i} \cdot \mathrm{e}$ next section.

\subsection{Optical Constants and Distortion Correc- tion}

The objective of the optical constants program ${ }^{7}$ is to determine best estimates for the camera pinholes and fiducial transformations, and to parametrize the optical distortions so that measured data can be corrected before geometrical reconstruction. The distortion correction procedure will be represented by the mapping $D C: R^{2} \rightarrow R^{2}$ from the film plane to itself. The fiducial system consists of a square distortion grid above the chamber at $z \simeq+43 \mathrm{~cm}$ and a set of fiducial marks below it at $z \simeq-40 \mathrm{~cm}$ [Alb83]. The need for distortion correction is evident from a CCD picture of the distortion grid. The central grid cells, which image onto the center of the optical assembly output phosphor, are nearly perfect squares. Neighboring cells suffer the pincushion distortion characteristic of simple, positive lenses [Hec87]. The curvature of the distortion reverses and becomes more intense at the edges of the image which coincide with the periphery of the image intensifier assembly.

The input to the calculation consists of surveyed positions of the distortion grid and the fiducial marks, the measured images of the grid points and fiducials, and transformed values of the grid points and fiducials under the mappings $P$ and $F$ of Section 4.2:

1. $\overrightarrow{x f}, \overrightarrow{x g}$ : the surveyed positions in chamber space of the fiducials and grids. These are known with a precision of $\simeq 200 \mu \mathrm{m}$ in space [Alb83].

2. $\overrightarrow{x f}_{\text {meas }} \overrightarrow{x g}_{\text {meas }}$ : the measured images of the fiducials and grids. These twodimensional vectors are known with a precision of $\simeq 1$ pixel.

3. $\overrightarrow{x f}_{\text {undist }}, \overrightarrow{x g}_{\text {undist }}$ : the ideal, projective images of the fiducials and grids in the film plane, for example, $\overrightarrow{x f}_{\text {undist }}=P(\overrightarrow{x f})$.

4. $\overrightarrow{x f}_{\text {dist }}, \overrightarrow{x g}_{\text {dist }}$ : the distorted images of the fiducials and grids in the film plane, obtained by distorting the ideal projective images in the film plane, $\overrightarrow{x f}_{\text {dist }}=D C^{-1}\left(\overrightarrow{x f}_{\text {undiat }}\right)$.

5. $\overrightarrow{x f}_{\text {trane }}, \overrightarrow{x g}_{\text {trans }}$ : the transformed measurements of the fiducials and grids in the film plane, for example, $\overrightarrow{x f}_{\text {trans }}=F\left(\overrightarrow{x f}_{\text {meas }}\right)$.

\footnotetext{
${ }^{7}$ The optical constants program, OPTICN35, was developed by P. Seyboth for experiment NA5, which employed the same streamer chamber. It has nerer been documented except in an NA5 internal note [Sey86]. The NA5 streamer chamber was also used by the EMC collaboration for experiment NA9 [Alb83] but with different optice.
} 
The CERN minimization package MINUIT [Jam89] is employed to vary three parameters, the coordinates of the pinhole $\left(c_{x}, c_{y}, c_{z}\right)$, subject to the constraint that the two angles which specify the optical axis vector $\vec{a}$ remain fixed. For each value of the parameters the fiducial transformation $F$ is found from a least-squares fit to the grid measurements. The fit minimizes the sum of the squared distances from the transformed measurements to the corresponding, undistorted, projective images in the film plane, assuming equal error's for all distances:

$$
\chi_{F}^{2}=\sum_{i=1}^{n g r i d}\left\|\overrightarrow{x g}_{\text {trans }}^{i}-\overrightarrow{x g}_{\text {undist }}^{i}\right\|^{2} .
$$

Approximately 120 grid measurements are used in the calculation. After $F$ has been determined the measured grid points are transformed to the film plane and a distortion table is computed. At each grid node the difference vector $\overrightarrow{d x}$,

$$
\overrightarrow{d x}=\overrightarrow{x g}_{\text {trans }}-\overrightarrow{x g}_{\text {undiat }}
$$

represents the local distortion. Transformed measurements are distortion -corrected by linearly interpolating between the four $\overrightarrow{d x}$ vectors at the grid nodes bounding the appropriate grid square.

The distortion correction and fiducial transformation are used to predict the images of the fiducial marks in measurement space. For each fiducial in chamber space,

$$
\overrightarrow{x f}_{\text {pred }}=F^{-1} \circ D C^{-1} \circ P(\overrightarrow{x f}) \text {. }
$$

The composition means mapping $P$ followed by $D C^{-1}$ followed by $F^{-1}$. MINUIT converges to values of the parameters $\left(c_{x}, c_{y}, c_{z}\right)$ which minimize the squared distances from the fiducial measurements to the fiducial predictions in measurement space:

$$
\chi_{\text {minimization }}^{2}=\sum_{i=1}^{n f i d u c i a l}\left\|\overrightarrow{x f}_{\text {meas }}^{i}-\overrightarrow{x f}_{\text {pred }}^{i}\right\|^{2} .
$$

The minimization is carried through separately for each camera. After the minimization is completed the final distortion table is evaluated. The distortion table is extrapolated to imageable regions of the chamber where the grid squares have been incompletely measured. At convergence, the rms deviation of the fiducial predictions from the measurements is $\simeq 0.5$ pixels, except for fiducials which are located in the extrapolated regions of the distortion table. The resolution in space can be estimated from the corresponding point reconstruction of the fiducial marks. The rms deviation in the $x y$ plane of the reconstructed fiducial positions from the surveyed positions is $\simeq 800 \mu \mathrm{m}$, consistent with the $S_{\text {eff }} / 10$ estimate discussed in Section 3.4. See Figure 3.10. The rms deviation in $z$, excluding fiducial marks which are measured in extrapolated regions of the distortion table, is $<2 \mathrm{~mm}$. Note that the lever arm for the intersection of light rays for the corresponding point reconstruction is $\simeq 370 \mathrm{~cm}$ at the fiducial plane. For virtually all imaged points, the overall resolution is $\simeq 2 \mathrm{~mm}$ in space. 
The first step in track reconstruction is the replacement of the measurements by their distortion-corrected images in the film plane $\vec{x}_{f p}=D C \circ F\left(\vec{x}_{\text {meas }}\right)$. Since the CCD image sensor has a fixed position with respect to the fiducial marks, the fiducial transformation $\boldsymbol{F}$ need only be calculated once, simultaneously and selfconsistently with the calculation of the distortion table. This single transformation is applied to all measured data. ${ }^{8}$

\subsection{Track "Measuring"}

By track measuring we mean the sequence of steps required to obtain a set of $(x, y)$ points in the measurement space. It is a combination of computer tracking, track filtering, and manual scanner cleanup. It was originally hoped, and is still the goal [Cha92], that the tracking would attain high enough efficiency to permit the extraction of certain physics observables without scanner assistance. For the NA35 CCD data reported in this thesis this was not the case. The track finding efficiency, estimated from visual comparison of the found tracks with CCD images, was $\simeq 70-80 \%$ at the stage of computer tracking used in this thesis. Let $p$ be the probability of finding a track in a single view. If tracking inefficiencies are independent from view to view, and if two-view reconstructions are included, then the reconstruction efficiency will be

$$
\eta_{\text {rec }}=p^{3}+3 p^{2}(1-p)
$$

This implied $\eta_{\text {rec }} \sim 80 \%$ including nearly half two-view reconstructions which was judged to be unacceptable, particularly in light of the traditional expectation of exceptionally high efficiency for both bubble chambers and streamer chambers with conventional or computer-assisted measuring. Moreover a distinction must be made between tracks that are found and tracks that are well measured. With this additional criterion, the track finding efficiency was reduced to $\sim 60 \%$.

After the initial computer tracking step, the digitized data are filtered. The main objectives of this pre-cleanup filter are to reject spurious tracks, remove outliers from valid tracks, and to ensure that all tracks selected for the next stage of processing are consistent with the measurement guidelines discussed in Section 4.4.3. The filtered data is selectively remeasured by scanners with the intention of achieving close to $100 \%$ efficiency in a good fiducial volume in the mesaurement space. A post-cleanup filter, which can reject outlier points but assumes all tracks are valid, improves the overall quality of the measured data, aids the training and supervision of the scanners, and calculates measuring distributions which characterize the departures of the data from ideal measurement. Simulated events, which take into account the limitations of the measuring, are constructed by sampling from these distributions.

\footnotetext{
${ }^{8}$ This is different from film. Because of film transport effects, the fiducial positions in filmimaged data vary from frame to frame and $F$ must be calculated on a frame-by-frame basis, independent from the calculation of the distortion table.
} 
The tracking will be described in detail in Ref. Cha92 so only a brief discussion will be presented here.

\subsubsection{Tracking}

Our approach to tracking evolved from our early success in track finding using a simple, one-dimensional peak-finding algorithm along linear contours. See Figure 3.11. Alternative methods which rely heavily on image processing techniques have been employed by NA35 with film data digitized offline by a high resolution, line-scanning CCD camera [Roh87], [Fle89]. We investigated standard image processing operations but they did not significantly suppress background nor were they particularly effective at locating streamers. The tracking takes place in four steps. First, streamers are located by a peak-finding algorithm which identifies track-contour crossings. Second, streamers are joined to form track fragmentscircular segments which pass through the primary vertex. Next, the fragments are merged into track candidates. Last, a small set of candidates is rejected to arrive at the final track set.

1. Peak-finding. We search for peaks along a set of nested contours devised to be approximately orthogonal to nearly all the tracks. Both circular contours concentric with the target and elliptical contours confocal with the target were tried. Compared to full-frame image processing operations, contour searches significantly reduce computing time and impose a predictable radial spacing on the candidate peaks which facilitates the overall tracking. Peakfinding is somewhat of a misnomer since the algorithm looks for ridges in the intensity distribution which correspond to track-contour crossings. The calculation is illustrated in Figure 4.6. The gradient vector of the intensity distribution $\nabla I(x, y)$ is calculated from finite differences. The directional derivative along the contour $d f / d l$ is approximated by the component of the gradient perpendicular to the radial line from the vertex to the candidate pixel $(\mathrm{CP}), d f / d l \simeq \nabla I(x, y) \cdot \hat{\mathcal{L}}$. The radius vector $\vec{r}$ and the unit vectors $\hat{\|}$ and $\hat{\perp}$ parallel and perpendicular to $\vec{r}$ are indicated in the figure. A ridge crossing occurs when the directional derivative changes sign. The intensity surface in the neighborhood of the $\mathrm{CP}$ is approximatud by a general quadratic function:

$$
I(x, y)=a x^{2}+b y^{2}+c x y+d x+e y+g .
$$

At an extremum the first derivatives must vanish. So in the neighborhood of a candidate track point, the intensity surface can be represented by the quadratic form:

$$
I(x, y)=a x^{2}+b y^{2}+c x y .
$$

The coefficients, which are second derivatives evaluated at the CP and describe the curvature of the surface, are again calculated from finite differences. A principal axis transformation identifies the major axis which by definition is the direction of minimum curvature. The principal axes $p_{1}$ and $p_{2}$ and representative level curves of the intensity ellipsoid are shown schematically in Figure 4.6.

The CP is called a peak if: 


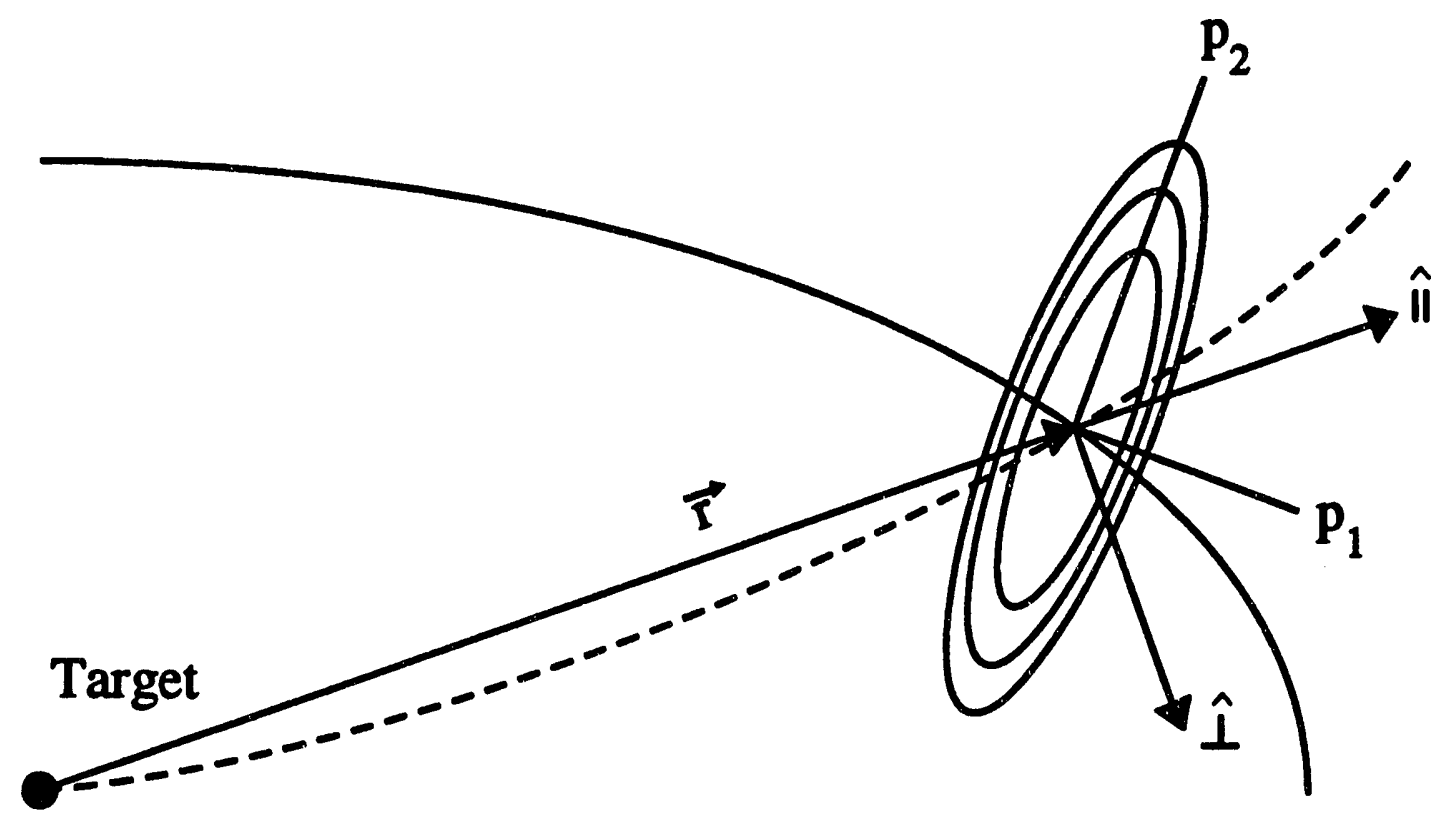

Figure 4.6: Peak-finding along elliptical contours. The track, shown as a dashed line, intersects the contour at the candidate pixel. The quadratic approximation to the intensity surface is represented by the elliptical level curves with respect to the principal axes $p_{1}$ and $p_{2}$. A ridge crossing occurs when $d f / d l \simeq \nabla I(x, y) \cdot \hat{\perp}$ changes sign. 
1. Its intensity, averaged over nine nearby pixels, is greater than the CCD offset plus local background.

2. The minimum curvature of the intensity surface, rescaled by the average intensity, is negative and large enough, indicating a maximum or a saddle point.

3. The gradient changes sign between the previous pixel on the contour and the CP.

The peak positions are determined by linear interpolation along the contour between the CP and the previous pixel. In this manner, the centroids of points along tracks can be found to better than the one pixel accuracy of hand measurements.

2. Fragment finding. Starting on the most distant contour from the target, where the track separation is the cleanest, and proceeding contour-by-contour towards the vertex, each peak serves as the seed for the growth of a set of fragment candidates. Only one peak per contour may be added to any candidate. Distinguishing features of our fragment finding are the use of recursion and the rooted tree data structures which are natural for a recursive search. The algorithm for selecting the search direction was adapted from a well-studied mothod used in radar tracking called the alpha-beta tracker [Cad73]. Each peak within an angle window about the search direction is added to the current candidate to form the next level of the tree and so on.

When the recursive search from a given peak is exhausted, each candidate is fitted to a circle. A $\chi^{2}$ and a figure-of-merit are defined:

$$
\begin{aligned}
\qquad \chi^{2} & =\frac{\sum_{i=1}^{n}\left(\sqrt{\left(x_{i}-x_{0}\right)^{2}+\left(y_{i}-y_{0}\right)^{2}}-r_{0}\right)^{2}}{n-2}, \\
\text { figure-of-merit } & =\frac{n}{\chi^{2}} .
\end{aligned}
$$

$\left(x_{0}, y_{0}\right)$ and $r_{0}$ are the center and radius of the fitted circle and $n$ is the number of points on the candidate fragment. Since the fits are constrained to pass through the vertex, they have only two degrees of freedom. An intensity comparison determines if the the average intensity per pixel is greater along the track candidate than along nearby, parallel trajectories. This test is weak and is intended to reject "matchsticks", short spurious tracks which accidentally point back to the vertex. A candidate is selected from the tree if:

1. It has the best figure-of-merit.

2. The $\chi^{2}<\chi_{\max }^{2}$, a cutoff estimated from circle fits to hand-measured data.

3. The intensity test is satisfied.

Successfully found fragments are extended by adding points. This procedure is virtually identical to the fragment search but takes advantage of the reduction in 
available peaks because of the assignment of peaks to tracks and the better predictions which result from more complete knowledge of the track parameters. Points are added only if they improve the fit or make a small change to an exceptionally good fit.

3. Fragment merging. A final set of track candidates is chosen by recursively merging track fragments into single tracks. Fragments which may lie along the same track are selected if they have approximately the same projected radius of curvature and angle of emission at the vertex. They are spliced into single tracks, their composite $\chi^{2}$ is calculated as above, and they are merged if:

1. The $\chi^{2}$ remains $<\chi_{\max }^{2}$.

2. The $\chi^{2}$ improves as a result of the association into a single track.

The number of tracks remaining after fragment merging is typically half of the fragments found in the earlier step.

4. Candidate rejection. The final track rejection is weak. Its primary objective is to expedite the scanner cleanup by eliminating tracks outside the fiducial volume or tracks which may be well found but still inadequately measured for track matching and reconstruction. Spurious tracks have presumably been rejected earlier in the track finding although the intensity test is repeated for the track as a whole with this in mind. Tracks are rejected if they lie mostly in the cone or if they have too short projected arc length. Peaks which have not been associated with tracks are saved and made available to the scanners and may be used instead of hand-digitized points during cleanup.

\subsubsection{Track filtering}

Track filtering has two objectives. First, it attempts to improve the overall quality and reconstructability of the data by removing outlier points and enforcing fairly regular spacing between measurements. Second, it calculates measuring distributions from the data which quantitatively characterize the departures of the data from ideal measurement. The filtering is an iterative procedure based on a circular track model in the measurement space. After an initial fit, the signed distance $d_{i}$ of each point from the track is calculated from $\left(x_{0}, y_{0}\right)$ and $r_{0}$ of the fit:

$$
d_{i}=\sqrt{\left(x_{i}-x_{0}\right)^{2}+\left(y_{i}-y_{0}\right)^{2}}-r_{0} \text {. }
$$

Figure 4.7 shows the distribution of distances and both single and double Gaussian fits to data which has been tracked then cleaned up by the scanners. The double Gaussian fit, although artificial, illustrates the point that the distribution is comprised of different event classes-the tails are contributed by tracks with small radii of curvature. If the most distant point has $d_{\max }<n \sigma_{\text {gauss }}$, where $n=2(3)$ for pre(post)-filter and $\sigma_{\text {gauss }}=0.7$ pixels is the standard deviation of the single Gaussian fit, the track is said to have converged. If not, the most distant point is deleted 


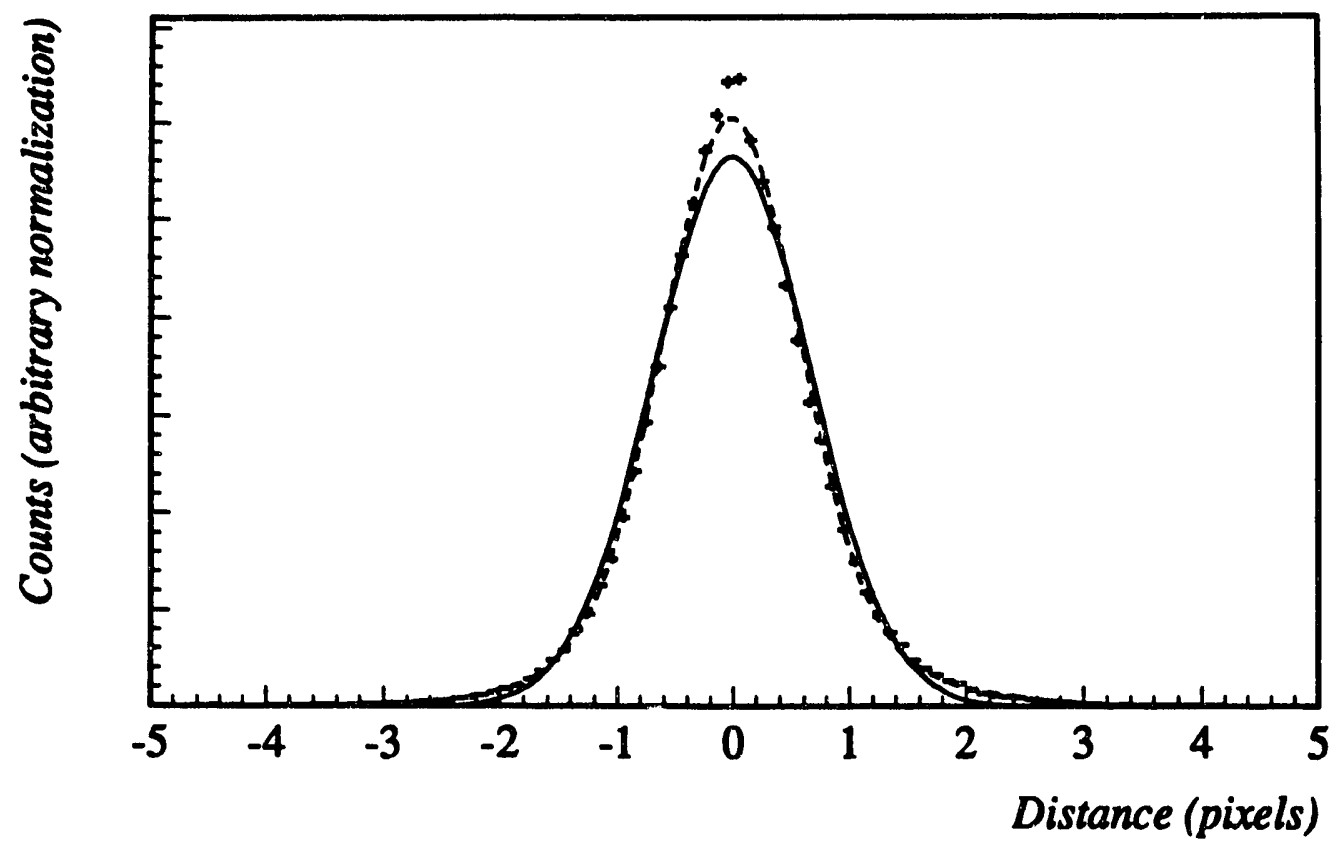

Figure 4.7: Deviation in pixels of measured track points from circle fits. The solid line is the result of a Gaussian fit, the dashed line of two Gaussians. The single Gaussian fit is taken to be an adequate representation of the data for filtering and simulation.

and the track refitted. The difference in the hardness of the cut before and after cleanup is indicative of our greater confidence in tracks which have been remeasured by the scanners. They are less likely to contain spurious points-relatively distant points are attributed to the tails of the distribution. Points are deleted until convergence or until the iteration is terminated. The maximum number of iterations is reduced for long, sharply curving tracks for which the circular model is a somewhat poorer global approximation. Most tracks converge after fewer than two iterations. Only $1.5 \%$ of tracks with $r_{0}>300$ pixels, which corresponds in the case of $\operatorname{dip} \lambda=0$ to $p \simeq 200 \mathrm{MeV} / c$, fail to converge. Visual examination of a large number of tracks has shown this procedure to be extremely powerful at rejecting outlier points which have been mistakenly or accidentally introduced during the measuring. The pre-filter deletes tracks if during iteration too few points or insufficient arc length remain for a satisfactory momentum reconstruction. Since the scanners are assumed to have conformed to the measurement guidelines listed in Section 4.4.3, no tracks are deleted by the post-filter.

After filtering, the tracks are pruned to remove points which are artificially close to preceding ones and to obtain quasi-equally spaced measurements. There are typically 30-50 measured points for a track with $\simeq 1 \mathrm{~m}$ projected arc length. The number of measured points per track is a strong but broad function of arc length. This is indicated in Figure 4.8, a profile histogram which plots the average and rms deviation of the number of measured points as a function of measured projected arc length $s p l$. The calculated arc length in pixels has been rescaled by 
the average camera demagnification to obtain distances $s p l$ in $\mathrm{cm}$. Also shown is a polynomial parametrization which is employed in track simulation to assign the number of points per track in a way consistent with the data. The improvement in measuring quality can be seen in Figure 4.9, a scatter plot of the rms deviation of the circle fits before and after filtering, denoted by $R M S 1$ and $R M S 2$, respectively. Because points are deleted randomly during the pruning, the rms deviation can increase for tracks which converge on the zeroth iteration. The output of the postcleanup filter constitutes the raw data which is the basis for track matching and momentum reconstruction.

In order to describe the departures of the measured data from ideal measurement a simple model was devised which addressed the most important features of measurement-scanning efficiency, track length and measuring error (generally referred to as setting error in the bubble chamber context). An ideally measured track possesses three attributes:

1. Its measured points are approximately equally spaced with at least 8 points for short tracks $(s p l \sim 20 \mathrm{~cm})$ and $\geq 30$ points for longer ones $(s p l \sim 100$ $\mathrm{cm})$. See Figure 4.8.

2. Its measured projected arc length would be the fully measureable length from its entrance point to its exit point in the fiducial volume. Obscuration of one track by another would not reduce the measured length.

3. The measuring error would be Gaussian distributed with $\sigma_{\text {meas }}<1$ pixel, the "least count" of our digitized data.

Attributes 1 and 2 minimize the inefficiencies due to failure of the track reconstruction, achieve the best momentum measurement because of the strong inverse power dependence of momentum resolution on arc length, and best constrain any matching procedure which relies on goodness-of-fit. Attribute 3 guarantees that the estimators minimized in the track fit are approximately $\chi^{2}$ distributed so that the fitting procedure is statistically sound. An ideally measured event would consist of ideally measured tracks with $100 \%$ scanning efficiency.

The distance distribution, which was plotted in Figure 4.7 and is the basis for the iterative filter, accounts for the measuring error. We introduce a fractional projected arc length distribution $f p l$ to account for the obscuration. Figure 4.10 illustrates its definition. The measured arc length $s p l$ is calculated in a straightforward manner from first to last measured point. The solid lines bounded by solid circles in the figure indicate the measured segments of tracks. Note that tracks can be extended into the cone if they can be resolved there. The arc length for the ideally measured equivalent track splmax is determined by extrapolating the measured segment back to the vertex and calculating the intersections of the extrapolated track with the four line segments indicated in the figure, which bound the good fiducial volume in measurement space. The extrapolated pieces of tracks are shown as dashed lines. The track-fiducial volume intersections are marked by open circles. In Chapter 5, the definition of the good fiducial volume is extended 


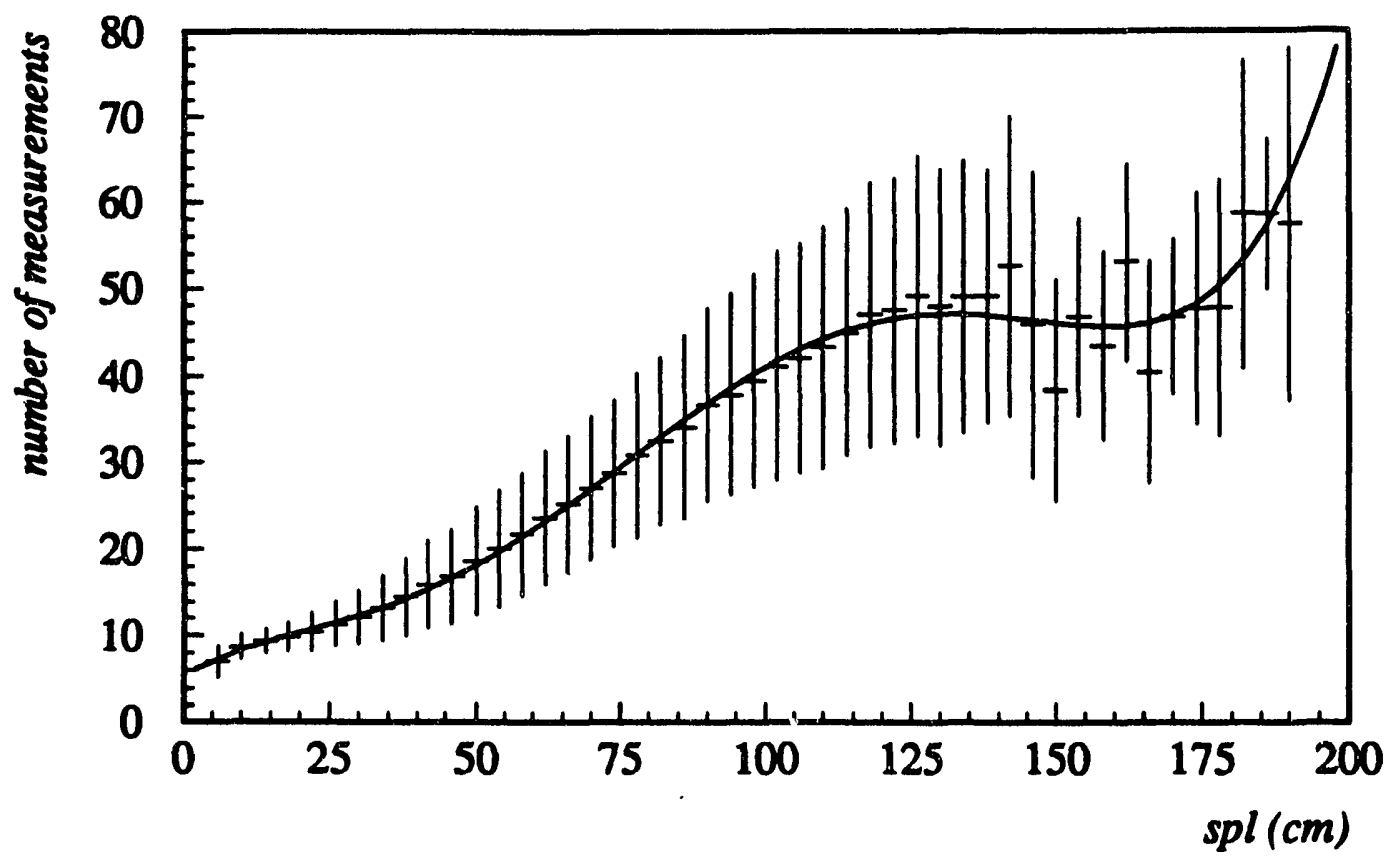

Figure 4.8: Number of measured points as a function of measured projected arc length spl. The profile histogram plots the mean and rms deviation of the underlying two-dimensional histogram. The solid line is a polynomial parametrization.

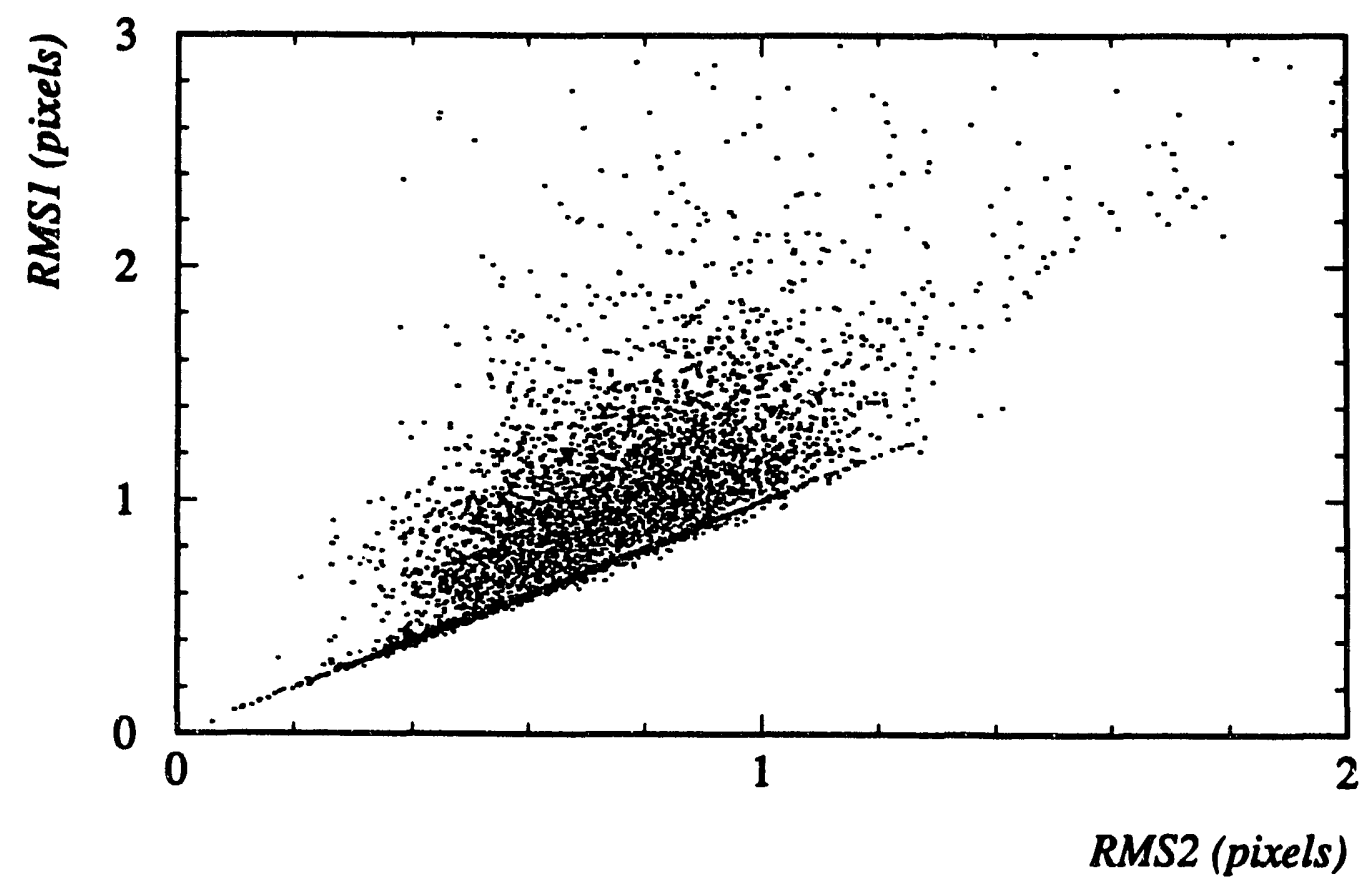

Figure 4.9: Rms deviation of circle fits. RMS1 and RMS2 are the respective deviations before and after the iterative filter. After convergence, all points are regarded as equally probable so random deletion of too-closely spaced points can lead to an increase in the deviation for tracks which converge on the zeroth iteration. 


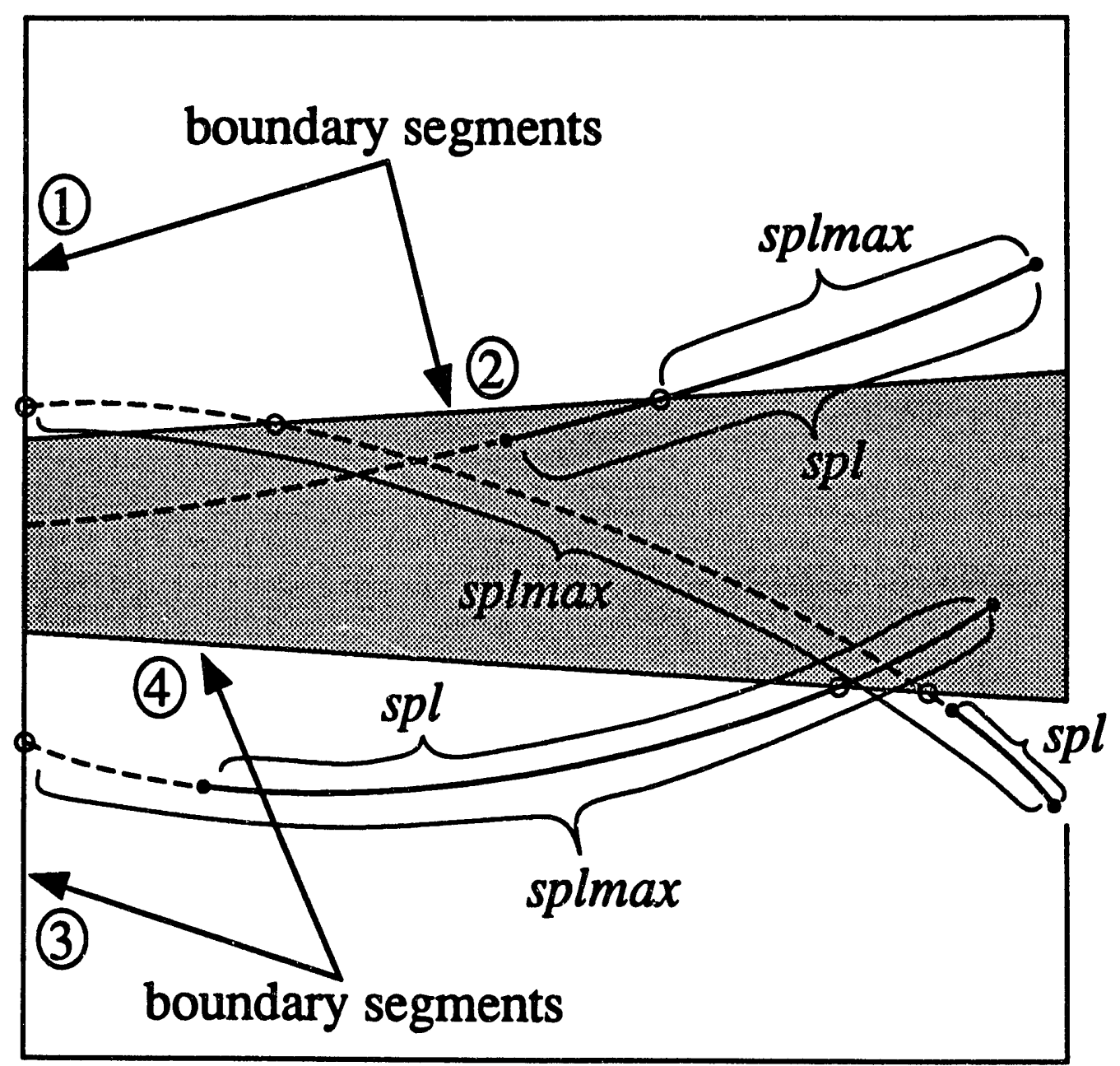

L

Figure 4.10: Definition of fractional projected arc length $f p l=s p l / s p l m a x$. The dark track segments indicate the measured portion of a track. The dashed lines are obtained by extrapolating back to the vertex. Track-fiducial volume intersections are identified by the open circles. The three examples shown have $n=1, n=2$, and $n=3$ intersections. The shaded region is the measuring cone. 
by the requirement that $s p l m a x>20 \mathrm{~cm}$ in all three views after reprojection of the trajectory of a reconstructed track into the measurement space. This can be done because splmax is independent of the measured arc length. Given a reasonable estimate of the vertex coordinates in measurement space and a well-measured track segment including the last measured point, splmax is completely determined, within the precision of the circle fit, regardless of how much of the track is measured or how much of the track is obscured.

The splmax distribution is shown in Figure 4.11. A rigid beam track, traversing

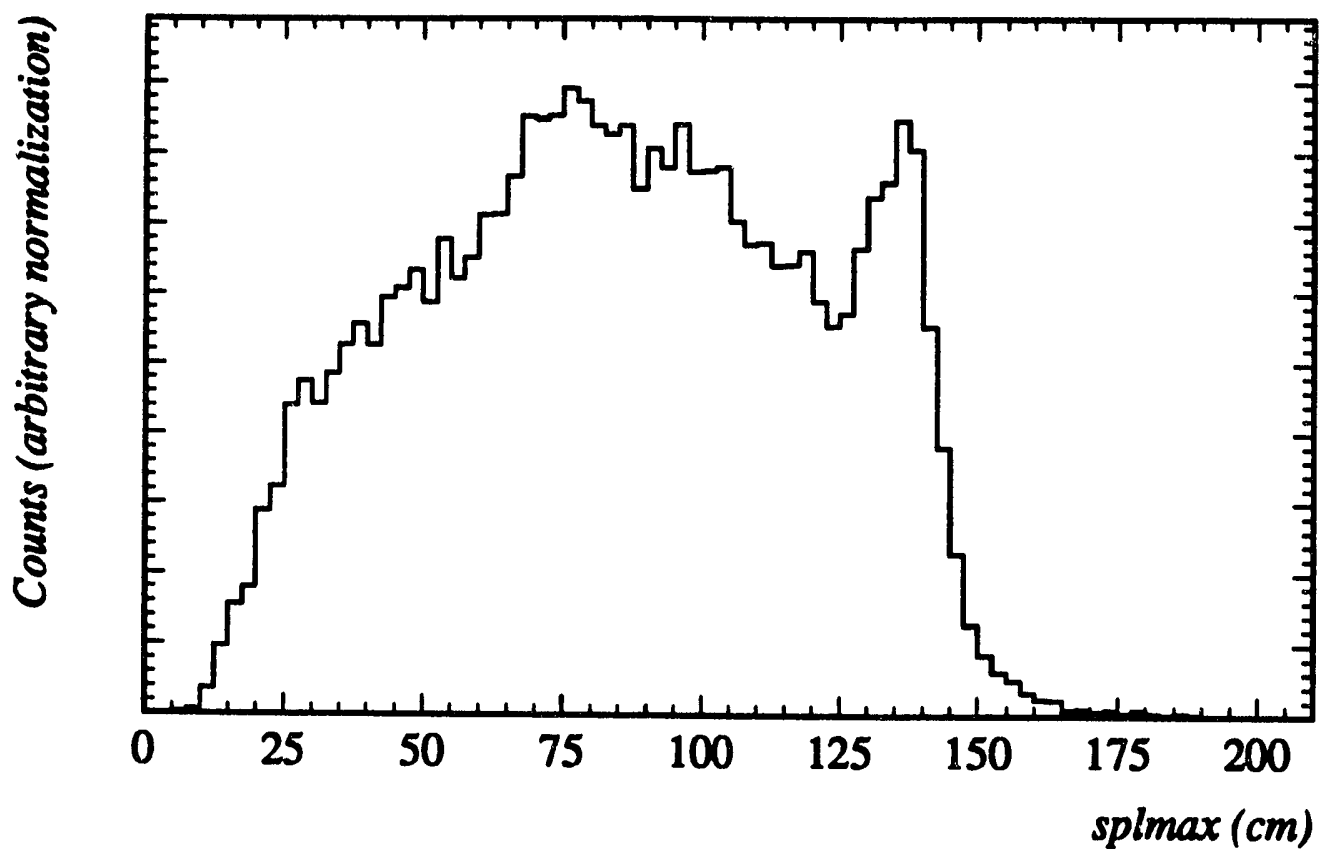

Figure 4.11: Maximum projected arc length distribution for equivalent, ideally measured tracks. The scanners were instructed to only measure tracks whose splmax exceeded the length of a flexible template which corresponded to $\simeq 15$ cm.

the subset of the streamer chamber within the optical field-of-view, would have $s p l m a x \simeq 130 \mathrm{~cm}$. The sharp peak for splmax large consists of wron gside tracks, some of which cross the cone. This distribution also demonstrates the degree to which the scanners adhered to the arc length requirement of the measuring guidelines and is the basis for the splmax $>20 \mathrm{~cm}$ arc length cut mentioned above which plays an important role in the geometrical efficiency correction discussed in Chapter 5.

For each track $f p l$ is defined in the obvious way:

$$
f p l=\frac{s p l}{s p l m a x} .
$$

Note that $f p l$ can be greater than 1 for tracks which are extended into the cone but do not cross it. Tracks can be characterized by the number of intersections 
$n=1,2,3$ or 4 which a given track can make with the boundary segments. Three examples are illustrated in Figure 4.10. Intuitively, one expects that tracks which enter then leave the cone, which can be identified by $n>2$, will be distributed differently than tracks which do not. One also expects an $f p l$ dependence on track kinematic properties, namely the rigidity and angle of emission at the vertex. In chamber space these correspond to the phase space variables $(p, \theta)$. In the measurement space the corresponding quantities are $\left(r_{\text {proj }}, \theta_{\text {proj }}\right)$, the projected radius of curvature and projected angle of emission.

The dominant dependence is on angle as indicated in Figure 4.12, a scatter plot of $f p l$ as a function of $\theta_{\text {proj. }}$. Because of the external target, the domain of $\theta_{\text {proj }}$ is restricted for the most part to $-\pi / 2<\theta_{\text {proj }}<\pi / 2$. The upper figure, labeled $n<3$, is for tracks which do not cross the cone. Note the reflection symmetry

about $\theta_{\text {proj }}=0$. The narrow tail out to $f p l=3$ for small $\theta_{p r o j}$ is contributed by tracks which emerge from the cone near the downstream end of the fiducial volume. Even though these typicaliy have short measured arc lengths, tracks of this type can sometimes be extended into the cone. For $n<3$, the fpl dependence on rigidity is weak. The lower figure, plotted for the case $n>2$ and which consists exclusively of wrongside tracks, has a completely different structure. Here, by definition, $f p l$ cannot exceed 1 and there is evidenced the necessary hole at small $\theta_{\text {proj }}$-tracks must initially be pointed outside of the cone for the magnet to bend them across it. Tracks cleanly divide into two classes at $f p l \simeq .8$, where there is a dramatic waist in the scatter plot. Cone-crossing tracks are either likely to be visible along their whole length leading to the sharp peak at $f p l$ approaching 1 , or likely to be obscured on one side or the other or measured as two separate tracks leading to the broad bump for $.2<f p l<.8$. There is a small asymmetry for $\theta_{\text {proj }}<0$. This is probably contributed by low momentum, wrongside, target protons which have no counterpart on the positive $\theta_{\text {proj }}$ side. For this case, the $f p l$ dependence on rigidity is very similar to the dependence on $\theta_{\text {proj }}$ because the kinematic variables are correlated for soft tracks. A good representation of the obscuration-dependence of the measured data is obtained from $n$ and $\theta_{\text {proj }}$ alone. The distributions in Figure 4.12 are projected onto the $f p l$ axis for distinct bins in $\theta_{\text {proj }}$ for the purpose

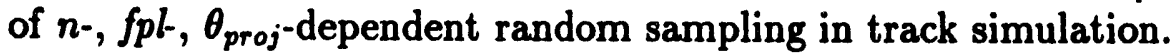

\subsubsection{Cleanup}

Scanner cleanup takes place in a completely digital environment we refer to as the scan station. The scan station is based on the graphics display device described in Chapter 3. A measuring session is completely driven by keystrokes entered on a 16key digitizing tablet. Track points can be hand-digitized at the single pixel integer resolution of the CCDs or cursor-selected from the floating point peaks left over from the tracking. Additional functions mapped to the tablet permit the scanner to effortlessly display, delete, or redraw tracks and points, to join track fragments into single tracks, or to enlist aid from the large class of software tools developed for the tracking, matching and reconstruction. Of particular utility are the optical tools which can project points or tracks from one view to another, or overlay the 

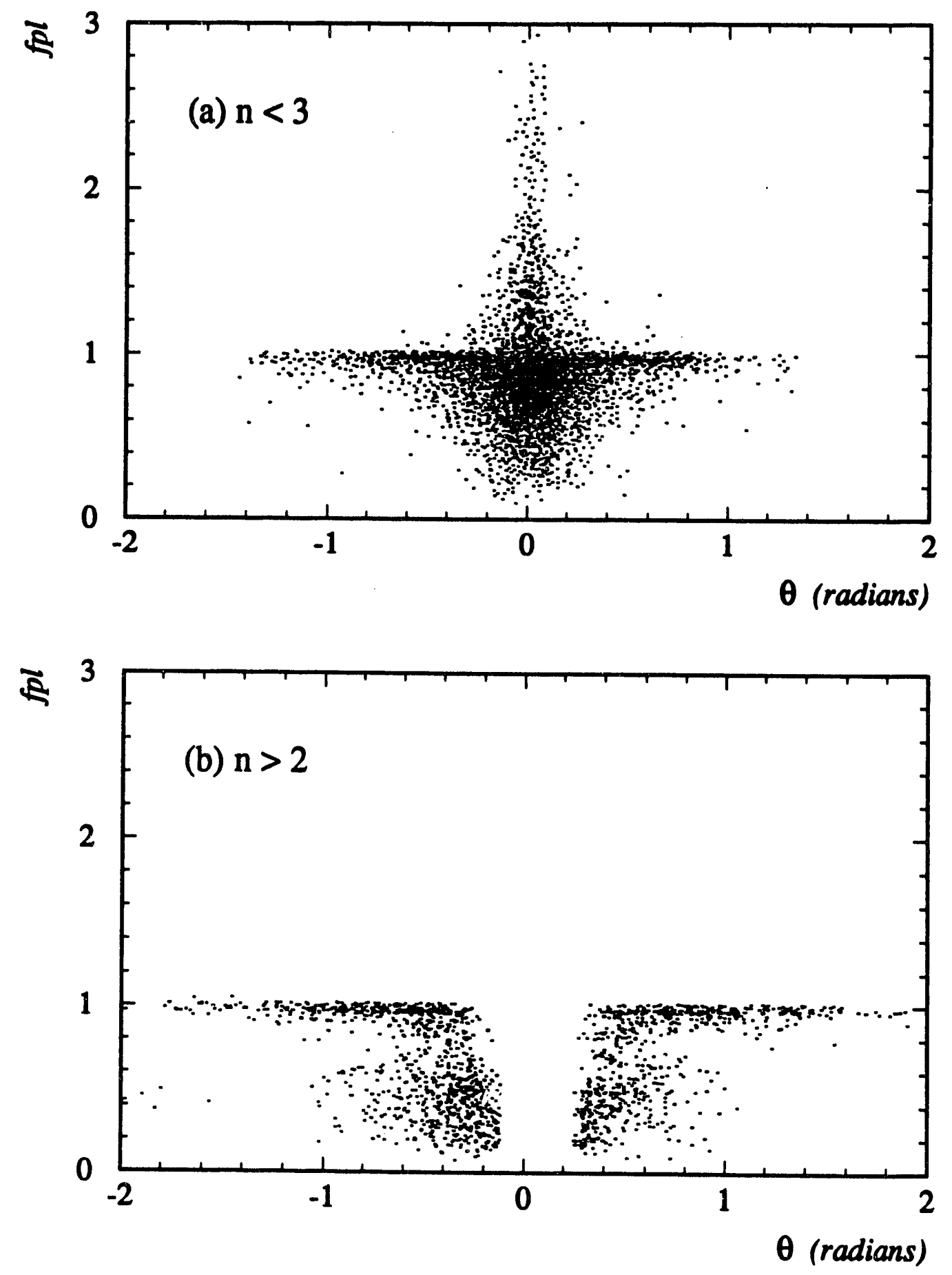

Figure 4.12: Fractional projected arc length $f p l$ as a function of $\theta_{\text {proj. }}$. The upper figure is for tracks which do not cross the cone, identified by $n<3$. The lower plot is for tracks which cross the cone. 
measurement space trajectories that would be associated with a given momentum fit. Virtually all the programs in the full data reduction chain can be executed from the scan station including single track two- or three-view reconstruction, full event matching, final reconstruction and vertex fit, and propagation of charged particles through magnetic fields. The scan station will be discussed in more detail in Ref. Cha92.

A well-measured track has an adequate number of quasi-equally spaced points, an $f p l$ close to 1, and approximately Gaussian distributed error. A well-measured view consists of well-measured tracks and has a scanning efficiency $\eta_{\text {ocanning }} \geq 95 \%$ and homogeneous measuring losses. By visually sampling a number of pictures a good fiducial volume was defined in the measurement space of each view, delineated by the square edges of the CCD and the boundary of the measuring cone. The measuring cone is the shaded region in Figure 4.10. The initial assumption was made that within this volume the scanning efficiency would be independent of position so an overall efficiency could be estimated by double scanning a set of events. As discussed in the next section, this assumption turned out to be untrue. The results of the double scan forced the good fiducial volume to be reduced-a larger cone, an analysis cone, was defined. The larger conecut was applied offline in software. Our philosophy was to measure the longest possible arc length, but to only measure tracks and points which were truly "seen", and to only expend precious scanner resources on track data which could be corrected later for the onset of our acceptance near the cone. This philosophy was imparted to the scanners by the following set of measuring guidelines:

1. Measure at least 8 points per track.

2. Only measure tracks which have splmax $>15 \mathrm{~cm}$ (see Figure 4.11.)

3. Measure outward to the last visible streamer and as far back toward the vertex as possible.

4. Extend tracks into the cone provided they satisfy 2.

5. Measure long, sharply curving tracks which cross the cone as single tracks if the fragments on either side of the cone can be associated with certainty. If not, measure as two tracks.

6. For spiralling tracks, only measure the branch which points back to the target. If this branch cannot be selected with certainty, measure each branch separately.

7. Measure overlapping tracks which merge and obscure each other to the extent which points can be assigned to one or both tracks with certainty.

8. Do not measure past kinks.

9. Do not measure tracks which clearly do not originate at the vertex. 
10. Never measure points which are uncertain.

The differences in interpretation of certain guidelines, for example rule 2 and 9 , can lead to an apparent inefficiency. Violations of rule 9 are generally rejected later in the data reduction. Tracks introduced by inconsistent application of rule 2 are rare (see Figure 4.11) and are rejected by the more selective offline cut splmax $>20$ $\mathrm{cm}$. However the discretionary interpretation of guidelines 5 and 6 may lead to biases. Tracks which cross the cone and cannot be associated into single tracks, if double measured in this way in more than one view, may result in two reconstructed tracks with nearly identical momenta. The introduction of such a spurious track is rare and will have a negligible effect on any single particle inclusive spectrum. In an intensity interference measurement, however, where the interesting part of a correlation function may depend on only a small number of track-pairs with small momentum differences, an effect of this kind can severely bias the result. Most spiralling particles have $p<100 \mathrm{MeV} / c$ which is a cut applied during the analysis.

The scanners were instructed to verify the correctness of tracks measured by the tracking program, bring them into compliance with the measuring guidelines, and measure the remaining tracks within the good fiducial volume. It required from 4-6 hours to clean up all three views of one event. The average charged multiplicity per view outside of the cone was $\simeq 90$. Over a 2 and $1 / 2$ year period 218 events were fully measured in three views requiring approximately one scanner-year of measuring effort.

\subsubsection{Scanning Ifficiency}

The method of double scanning for the estimation of the scanning efficiency and the true number of observed events has been applied to visual detectors for many years, dating in principle from 1924 when it was employed by Geiger and Werner in the visual counting of alpha scintillations [Aus69]. The formalism has been elucidated in a number of papers [Eva67], [Aus69], [Aus76]. A simpler method of estimation, which gives approximately the same result as the more sophisticated approaches without resolting to numerical evaluation, is outlined in Ref. Ead71. All the methods make the same three fundamental assumptions:

1. An event is either seen or not seen.

2. The two scans are independent.

3. All events are equally likely to be seen.

In our context replace event with track. However, then the assumptions do not strictly apply. The measurement of a track, unlike the observation of an event having occurred in a bubble chamber, is not a Bernoulli trial. Tracks can be identically measured along a significant fraction of their arc lengths in two independent measurements then wander into disagreement. More problematic are cases where two different interpretations of close-crossing tracks are possible. An example of this chromosome defect is illustrated in Figure 4.13. Because events are tracked 


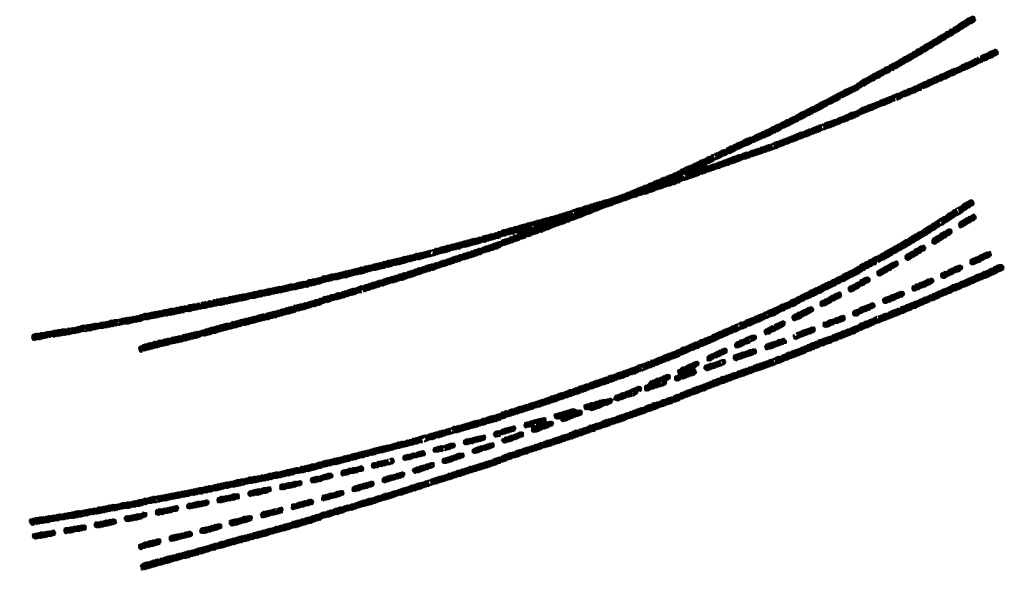

Figure 4.13: Chromosome measuring defect. The dark lines in the upper and lower track-pairs indicate the two possible interpretations.

before scanner cleanup, the two scans are not entirely statistically independent. And the validity of the assumption of homogeneous efficiency must be concluded from the double scan itself.

Events selected for double scanning had an average multiplicity $\bar{n}=92$ with no multiplicity smaller than 84 . This is to be compared with $\bar{n}=88,98,88$ in views 1,2 and 3 respectively calculated for the complete data sets. View 2, which achieved the best optical resolution and imaged a larger phase space slice near midrapidity, had a systematically higher multiplicity. Note that these numbers may differ significantly from the multiplicity distribution of reconstructed tracks. For track measuring the efficiency must be calculated from the set of imageable tracks. Since each camera views a slightly different part of the chamber and since tracks which are imageable in one view may flip into the cone in a different view, only a subset of the imageable tracks falls in the three-view acceptance.

Each scanner started with the same raw data, a CCD image plus the output of the tracking for each frame. After the scanners finished cleanup, the doublescanned events were meticulously reviewed track-by-track. The estimate of scanning efficiency requires the classification of each track as:

1. Seen by A but not by B.

2. Seen by B but not by A.

3. Seen by both $\mathrm{A}$ and $\mathrm{B}$.

4. Seen by neither A nor B.

The last set, which of course cannot be deduced from the data, closes the set of possible outcomes. After the assignment of every track into the correct category, the scanning efficiency can be calculated for each scanner. If $a$ and $b$ are the number 
of tracks found by scanners A and B in the double scan and if $\gamma$ is the number of tracks found in common then from the arguments given in Ref. Ead71 an estimate of the true number of tracks $\bar{N}$ is $\bar{N}=a b / \gamma$ which leads to the estimate of the efficiencies of the two scans:

$$
\begin{aligned}
\eta_{A} & =a / \bar{N}=\gamma / b \\
\eta_{B} & =b / \bar{N}=\gamma / a .
\end{aligned}
$$

Because track measurements don't strictly conform to assumption 1, classifications 1, 2, and 3 above require definition. First, a class of tracks which could lead to apparent inefficiencies was rejected from the calculation. Short stubs could be inconsistently measured because of different interpretation of the minimum arc length cut. These were rejected if their computed $s p l m a x<20 \mathrm{~cm}$. Similarly for inconsistent exclusion of nonvertex tracks-sometimes a clear nonvertex $V^{0}$ would be measured by one scanner and not another. Tracks could be double measured identically because of scanner oversight. Only one track from such a pair was included in the accounting. Finally, one scanner and not the other could successfully extend a track across the cone. This is a more subtle form of double counting. Again only one track from such a pair was retained in the analysis. We assume doubly measured tracks of both kinds, which are certainly in the data set, lead at worst to a very small introduction of spurious tracks but not to inefficiencies in matching and reconstruction. Chromosome pairs are considered inefficiencies for both measurements in the double scan; that is, they are assigned to the class "seen by $A(B)$ but not by $B(A)$." Their presence in the data can lead to two effects, either a failure of reconstruction or the introduction of a spurious track. However a spurious track of this kind should "remember" the kinematic variables of the correct track that escaped accurate measurement and is unlikely to distort a single particle inclusive spectrum. However, this problem illustrates again the difficulty of making an intensity interference measurement when subtle problems of measurement may induce correlations. Both for estimating scanning efficiency and for event simulations which incorporate this estimate, chromosomes are regarded as tracks which have been omitted.

Figure 4.14 shows a composite event of the five frames double scanned in view 1 , displaying only those tracks which were not correctly measured by both scanners. The cone, bounded by solid lines, is a subset of the shaded region labelled partition 1 in the figure. As is clear from the figure most of the losses are at the edges of the cone. The exceptions are the result of high multiplicity fluctuations which lead to clusters of tracks which superimpose in at least one camera projection and exceed the optical resolution. To properly apply the formalism the losses must be homogeneous so events "need to be grouped into bins of the parameters within which the intrinsic probability is likely to remain constant." [Eva67] As for the case of the measuring distributions, the efficiency should depend on the kinematic variables through the corresponding measurement space variables $r_{p r o j}$ and $\theta_{\text {proj }}$. With a statistically large enough sample, one could parametrize the efficiency as a smooth function of $\left(r_{p r o j}, \theta_{\text {proj }}\right)$. This is impracticable. A cruder approach is to partition the space into multiple regions and postulate homogeneous losses within 


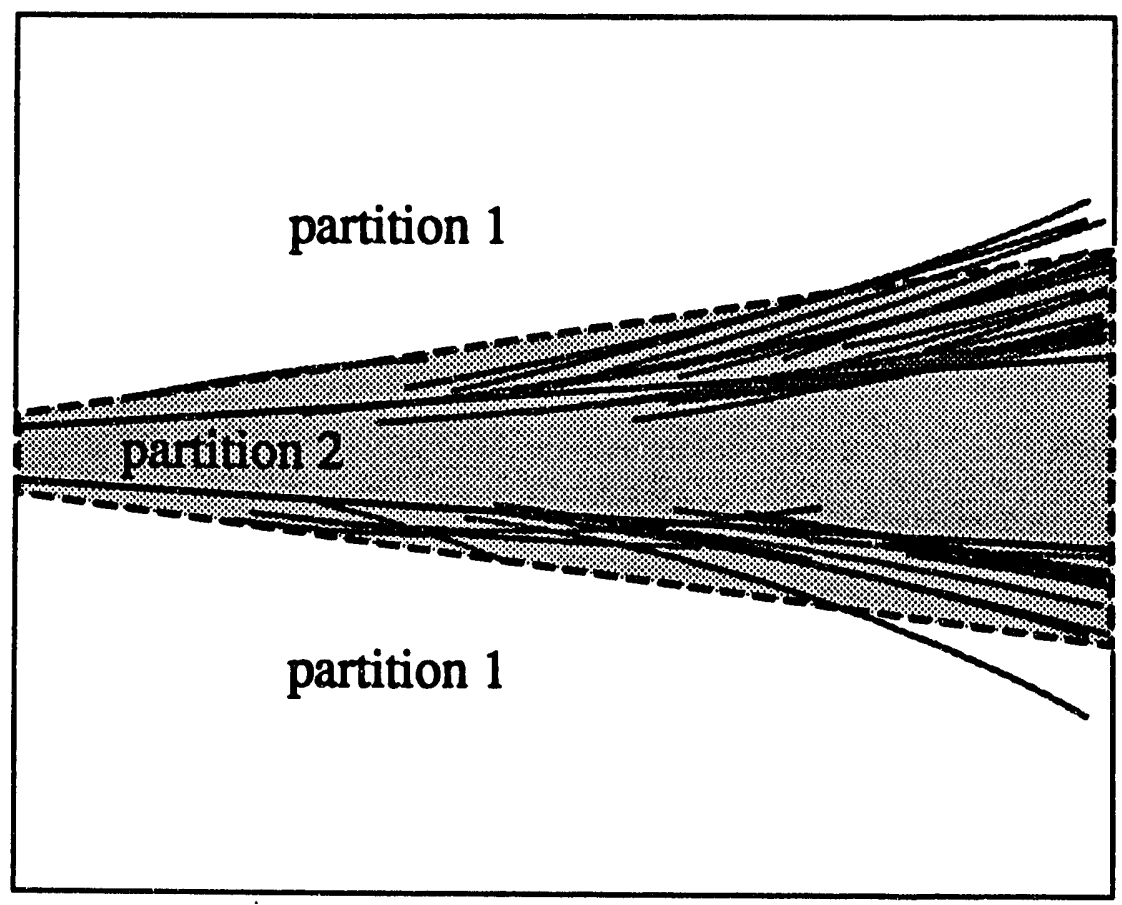

$L$

Figure 4.14: Composite event of all tracks in classifications 1 and 2, "seen by $A(B)$ but not by $B(A)$." The shaded region is partition 2, the remainder of the measurement space is partition 1 . The central cone is a subset of partition 2. 
each region. The two partitions adopted are also shown in Figure 4.14. A track is assigned to partition 2 if $>75 \%$ of splmax lies within the partition. This arc length requirement softens the seemingly hard edge dividing the partitions.

Each track in the double scan is assigned to a partition and the scanning efficiency is calculated from Equation 4.21 for each region independently. The results for view 1 are summarized in Table 4.1. Of $n_{\text {meas }}$ measured tracks, $n_{\text {ace }}$

\begin{tabular}{||c|c|c|c|c|c|c|c|c||}
\hline \hline \multicolumn{10}{||c|}{ Scanner $\mathrm{A}$} \\
\hline event & $n_{\text {meas }}$ & $n_{\text {acc }}$ & $n_{1}$ & $n_{2}$ & $\gamma_{1}$ & $\gamma_{2}$ & $\eta_{1}$ & $\eta_{2}$ \\
\hline 1 & 89 & 88 & 59 & 29 & 59 & 23 & 0.967 & 0.885 \\
\hline 2 & 93 & 92 & 64 & 28 & 63 & 23 & 1.000 & 0.852 \\
\hline 3 & 92 & 90 & 61 & 29 & 59 & 27 & 0.952 & 0.871 \\
\hline 4 & 103 & 101 & 75 & 26 & 74 & 11 & 1.000 & 0.478 \\
\hline 5 & 95 & 94 & 67 & 27 & 67 & 21 & 1.000 & 0.724 \\
\hline all & 472 & 465 & 326 & 139 & 322 & 105 & 0.985 & 0.772 \\
\hline
\end{tabular}

\begin{tabular}{||c|c|c|c|c|c|c|c|c||}
\hline \multicolumn{6}{||l}{ Scanner B } \\
\hline event & $n_{\text {meas }}$ & $n_{\text {acc }}$ & $n_{1}$ & $n_{2}$ & $\gamma_{1}$ & $\gamma_{2}$ & $\eta_{1}$ & $\eta_{2}$ \\
\hline 1 & 92 & 87 & 61 & 26 & 60 & 22 & $1.017^{9}$ & 0.885 \\
\hline 2 & 91 & 90 & 63 & 27 & 62 & 24 & 0.969 & 0.857 \\
\hline 3 & 93 & 93 & 62 & 31 & 59 & 27 & 0.967 & 0.931 \\
\hline 4 & 99 & 97 & 74 & 23 & 74 & 11 & 0.987 & 0.423 \\
\hline 5 & 99 & 96 & 67 & 29 & 66 & 22 & 0.985 & 0.815 \\
\hline all & 474 & 463 & 327 & 136 & 321 & 106 & 0.985 & 0.763 \\
\hline \hline
\end{tabular}

Table 4.1: Calculation of partition-dependent scanning efficiency. The subscripts 1 and 2 refer to partitions 1 and 2. The efficiencies $\eta_{1}$ and $\eta_{2}$ are calculated from Equation 4.21.

entered into the analysis after the exclusions outlined above. $n_{1}$ and $n_{2}$ were the number of tracks assigned to the different partitions, $\gamma_{1}$ and $\gamma_{2}$ the number in each partition measured by both scanners. The efficiencies $\eta_{1}$ and $\eta_{2}$ were calculated from Equation 4.21. Comparable results were obtained for views 2 and 3, in spite of differences in optical quality between the different views. Since the quantitative estimates for all three views were virtually identical, the distinction can be dropped leading to a single estimate of our scanning efficiency:

$$
\eta_{\text {acanning }}= \begin{cases}98 \% & \text { partition 1 } \\ 75 \% & \text { partition 2 }\end{cases}
$$

The statistical errors are $\sim 5$ and 10\%, respectively, in partitions 1 and 2 . The poor scanning efficiency in partition 2 biases the rapidity distribution which forces

\footnotetext{
${ }^{9}$ Although $\gamma_{1}+\gamma_{2}=\gamma$, the number of tracks seen by both $A$ and $B$, must be the same, differences in measurement can occasionally lead to a different partition assignment and an apparent efficiency $\eta>1$.
} 
us to reduce our acceptance. This issue is addressed in Section 5.2, particularly in Figure 5.4.

\subsection{Simulation}

Event simulation was used extensively during the development and debugging of the data reduction procedure and throughout the data analysis. Besides verifying the correctness of the matching and reconstruction codes, elements of the simulation chain were employed to calculate the matching and reconstruction efficiency, to calculate the acceptance of our measurement and associated geometrical corrections to the data, to determine the momentum resolution, to make modeldependent physical corrections, and to generate predictive overlays during track measurement. Finally, simulated data were used to define a number of important cuts applied in the track matching and in the final selection of data for analysis.

No attempt was made to simulate the ionization along charged particle trajectories, the streamer formation and light emission process in the chamber, or CCD response. Such an approach would have been required to create gray-level simulated images of streamer chamber pictures and was carried through for film data by other members of the NA35 collaboration [Gaz87]. However, the results of double scans performed on these images to estimate the efficiency for the detection of $V^{0}$ vertices were controversial [Ody90] because the images were not perceived as faithful replicas of genuine film events. Our objective was to create sets of points in the CCD measurement space which were a close approximation to the output from computer tracking or careful track measurement of CCD images and which incorporated the measuring limitations in a way which could be defined from the data themselves.

All the results presented in this chapter are based on purely optical simulations. A number of important physical effects have been omitted: the contamination of charged hadron spectra by electrons or by neutral strange particle decay products, multiple scattering, mainly in the target and the entrance window of the streamer chamber, which may influence vertex fitting and the ability to separate vertex from non-vertex tracks, and energy loss, again mainly in the target and entrance window. These will be addressed in Chapter 5 .

The Lund-FRITIOF Monte Carlo program for simulating nucleus-nucleus collisions [And87], [Nil86] was employed to generate events. The main requirement of any event generator for the results presented in this chapter is the ability to construct events with approximately correct total charged multiplicity and phase space distributions. This will result in simulated data with the correct topology in the measurement space, in particular the correct projected track density. The FRITIOF input parameters which specify nuclear geometry were tuned to reproduce the measured total inelastic cross section for ${ }^{32} \mathrm{~S}+{ }^{32} \mathrm{~S}$ of $\sigma_{\text {tot }}=1.8$ barns. Event classes were selected from impact parameter $b$ by $b d b$ weighting from $b=0$ to $b_{\max }$. From the measured cross section for a specific trigger $b_{\max }$ can be calculated assuming the cross section for clean cut geometry $\sigma_{\text {cleancut }}=\pi b_{\text {max }}^{2}$. Since 
the charged multiplicities resulting from this procedure were systematically low, $b_{\max }$ was reduced to produce events which conformed in multiplicity to the measured data. This inconsistency was rectified by including charged particles from $\gamma$ conversion in the target, from weak decays, and from secondary hadronic interactions in the target and detector material. See Section 5.4. Charged particles retained for the optical simulations were $\pi^{+}, \pi^{-}, K^{+}, K^{-}, p$ and $\bar{p}$, with all particles emitted from a primary vertex positioned $8 \mathrm{~cm}$ upstream of the entrance window to the streamer chamber.

Charged particles are propagated through the real NA35 magnetic field by numerically integrating the Lorentz force equation. The Cartesian coordinates of the field were obtained by mapping the field in approximately $5 \mathrm{~cm}$ steps. The mesh of nearly 28,000 measurements extended well into the fringe field region with an error of less than $\pm 20 \mathrm{G}$. The space variation of the field is considerable. Figure 4.15 shows representative field profiles of the radial and main field components $B_{r}(r, z)$ and $B_{z}(r, z)$ at two distances $z$ from the chamber midplane. The distance, $r$, is measured from the axis of symmetry of the Helmholtz coils of the superconducting magnet. Since the target is located outside of the streamer chamber fiducial volume at $r \simeq 108 \mathrm{~cm}$, all particles travel significant distances through a rapidly varying field. Field values at particular space points are determined by three-dimensional linear interpolation.

For the optical simulations the numerical integration was carried out by a swim program that was a modification of code used by the Alvarez group at the LBL $\mathbf{7 2}^{\prime \prime}$ bubble chamber [Ren90]. The precision of the swim was estimated for two cases: by comparing the results of the swim to an analytic swim for the case of a uniform field and by comparing to the Runge-Kutta particle tracking in GEANT [Bru87], the CERN detector simulation package, for the actual NA35 field map. For more than $99 \%$ of the charged particles in our acceptance, both the position of the fiducial volume exit point and the integrated arc length from the primary vertex to the exit point can be calculated with a precision better than $1 \mathrm{~mm}$. Except for employing the same field map, the momentum reconstruction and event simulation propagate particles through the field independently, providing an important consistency check for the reconstructed track momenta. The swirn program referred to in this section was used in the acceptance calculations and the geometrical weight calculations discussed in Chapter 5.

Approximately equidistant points in chamber space are obtained from the numerical integration by spline interpolation in arc length along the calculated trajectories. These points are projected to the measurement space along straight rays which pass through the equivalent camera pinholes. Distortion is added and the distorted points are mapped to the measurement space by the inverse of the fiducial mapping used in track reconstruction. See Equation 4.14. The subset of tracks which is imageable in a given view is determined by calculating splmax according to the prescription described in Section 4.4.2. An imageable track is defined as one with splmax $\geq 15 \mathrm{~cm}$, consistent with the measuring guidelines and the measured data (see Figure 4.11). A track is said to be in the three-view acceptance if it is imageable in all three views. For the matching problem, imageable tracks 

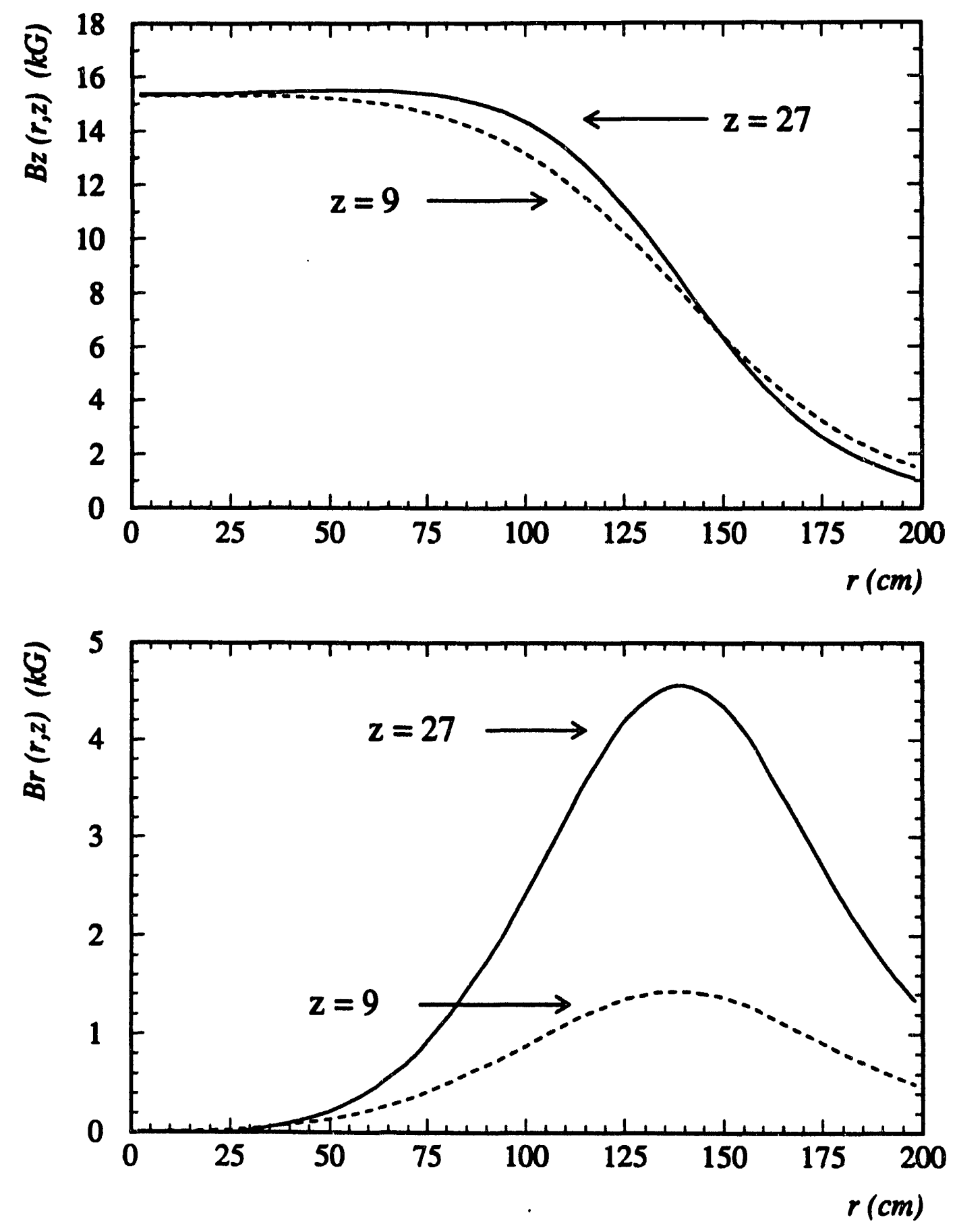

Figure 4.15: Magnetic field profiles. The main field component lies along the $z$ axis, $r$ is measured from the magnet axis of symmetry, and $z$ is measured from the chamber midplane. The external target is positioned at $r \simeq 108 \mathrm{~cm}$. 
which cannot be reconstructed or do not fall in the three-view acceptance can be considered as noise leading to the introduction of spurious tracks and the loss of valid image associations. The view-dependent final subset of tracks is selected by folding in the partition-dependent scanning efficiency. The measured arc length is assigned by randomly sampling from the appropriate $f p l$ distribution; the number of points is determined from the parametrization plotted in Figure 4.8. A set of approximately equidistant points in measurement space lying along the projected trajectories is obtained by random spline interpolation within bins whose number is commensurate with the number of points. Measuring error is added by sampling from the single Gaussian fit to the data shown in Figure 4.7.

A number of subtle measuring deficiencies have been omitted from the track simulation. No chromosome pairs were constructed from close-crossing tracks. Tracks which crossed the cone were never severed in two as if double measured. In the simulations it was assumed that tracks would be measured to their endpoints. The effect of the fpl-distribution sampling was to trim arc length from the beginning. In spite of the fact that scanners were instructed to measure tracks to the edges of the measurement space or, for stopping and punch out tracks, to their endpoints, they could not always do so.

Two flavors of simulation were employed in the analysis. A set of reference events, which retained only tracks in the three-view acceptance and assumed $100 \%$ scanning efficiency, was constructed and used to define the optimal solution to the matching and reconstruction problem. A set of realistic events was compiled according to the complete event simulation procedure outlined above. The quantitative estimates discussed in Section 4.8 were based on track-by-track comparisons with the reference sample.

Visually, simulated events are virtually indistinguishable from the measured data. Another example of the agreement of the simulated events with the data is indicated in Figure 4.16, a histogram of the rms deviation in the ideal film plane of the data points from the fitted tracks for both reconstructed, measured data and reconstructed, simulated data. The two histograms have been approximately normalized at their peaks. Only tracks which have been matched and reconstructed in three views are compared. The two-view data, which comprises $20 \%$ of the full data sample, has a similar distribution with a peak shifted slightly downward. The simulated data is well fitted by a Gaussian as is the measured data out to rms deviations of $\simeq 20 \mu \mathrm{m} . .^{10}$ The broadness of the distribution for the measured data compa. I to the simulation is probably a result of two factors: the tail in the measuremenc distribution of Figure 4.7, which was neglected in the single Gaussian error model for the simulation, and uncertainties in the optical constants and distortion correction which affect the data, but not the simulation. Although the measuring error is added after the distortion and inverse fiducial transformation, the simulated data points remain close enough to their pre-error positious that the

\footnotetext{
${ }^{10}$ Since the deviations are distributed normally, $\sqrt{\sum_{i}^{n} d_{i}{ }^{2}}$ should follow the $\chi(n)$ distribution [Ead71] which is asymptotically normal for $n>30$, a condition satisfied by almost all three-view reconstructed tracks.
} 


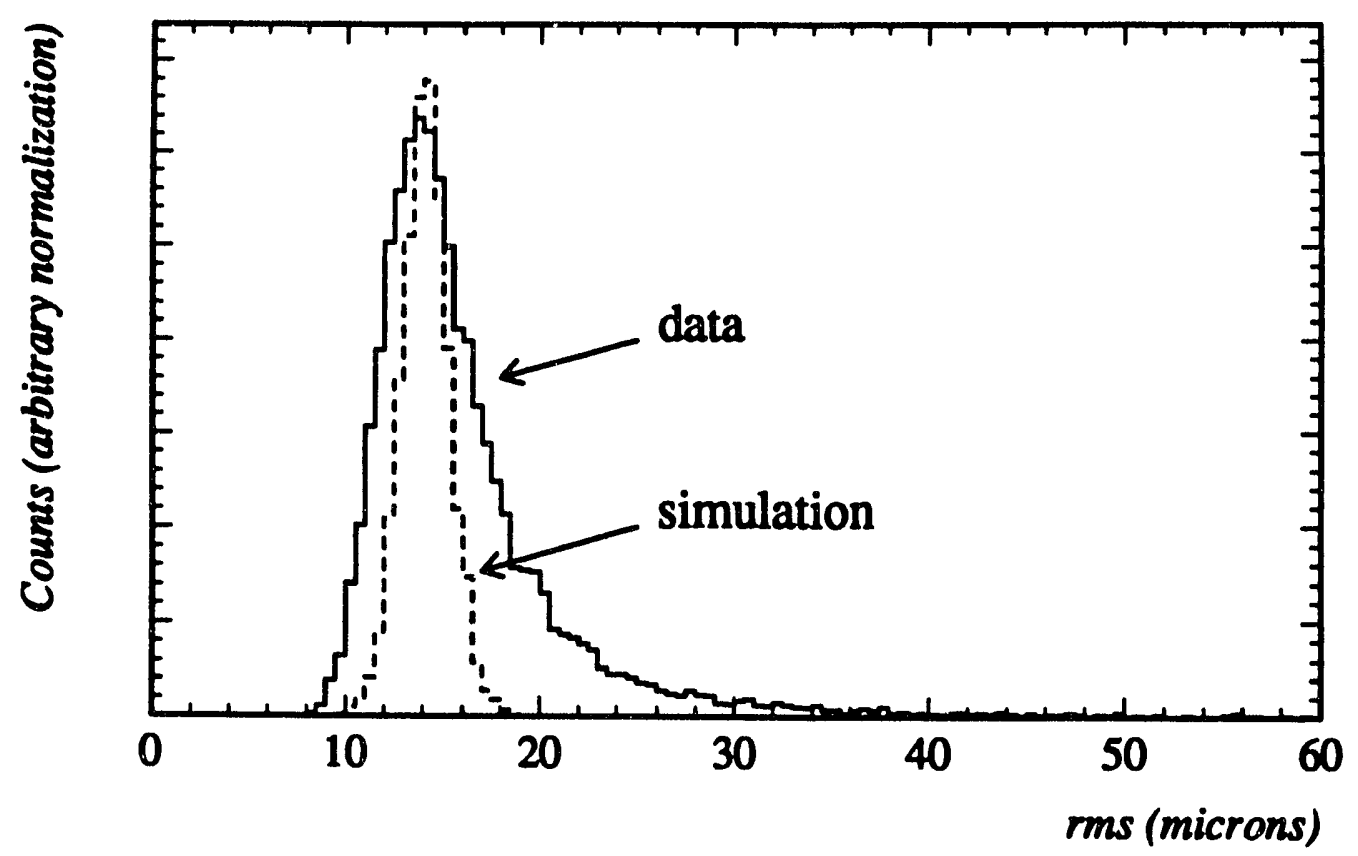

Figure 4.16: Rms deviation of data points from fitted tracks in the film plane. The three-view subset of the measured data, which comprises $80 \%$ of the total, is compared to the three-view acceptance reference sample.

distortion that is put in is very nearly what is taken out. Both distributions are peaked at $\mathrm{rms}=14 \mu \mathrm{m}$, the mean value of the Gaussian fits. The measurement error parameter in the reconstruction code, which along with the camera demagnification determines the scale of all error estimates deduced from the track and vertex fitting, was set to $15 \mu \mathrm{m}$ which resulted in track fits with a reduced $\chi^{2}$ distribution peaked very close to 1 .

Since reconstruction of track candidates is an integral part of the track matching, the reconstructed three-view acceptance reference events were used to define a number of cuts designed to reject spurious tracks:

1. Acceptance. The maximum momentum which can be measured in the good fiducial volume is $p_{\max }=16 \mathrm{GeV} / c$. Adding the vertex to short tracks can lead to artificial, excessively rigid tracks which are unphysical.

2. Goodness-of-fit. Upper bounds for the reduced $\chi^{2}$ of the track fit and the momentum resolution $\delta p / p$ were deduced from the tails of the corresponding distributions for the simulated data.

3. Physical start point. The start point of the track fit $\left(x_{0}, y_{0}, z_{0}\right)$, projected into a plane perpendicular to the beam and passing through the target, must be within bounds consistent with the limitations of the measuring. (See Section 4.2.)

The start point cut depends on whether the track is reconstructed in two or three views. This is illustrated in Figure 4.17, the distribution of the projected 


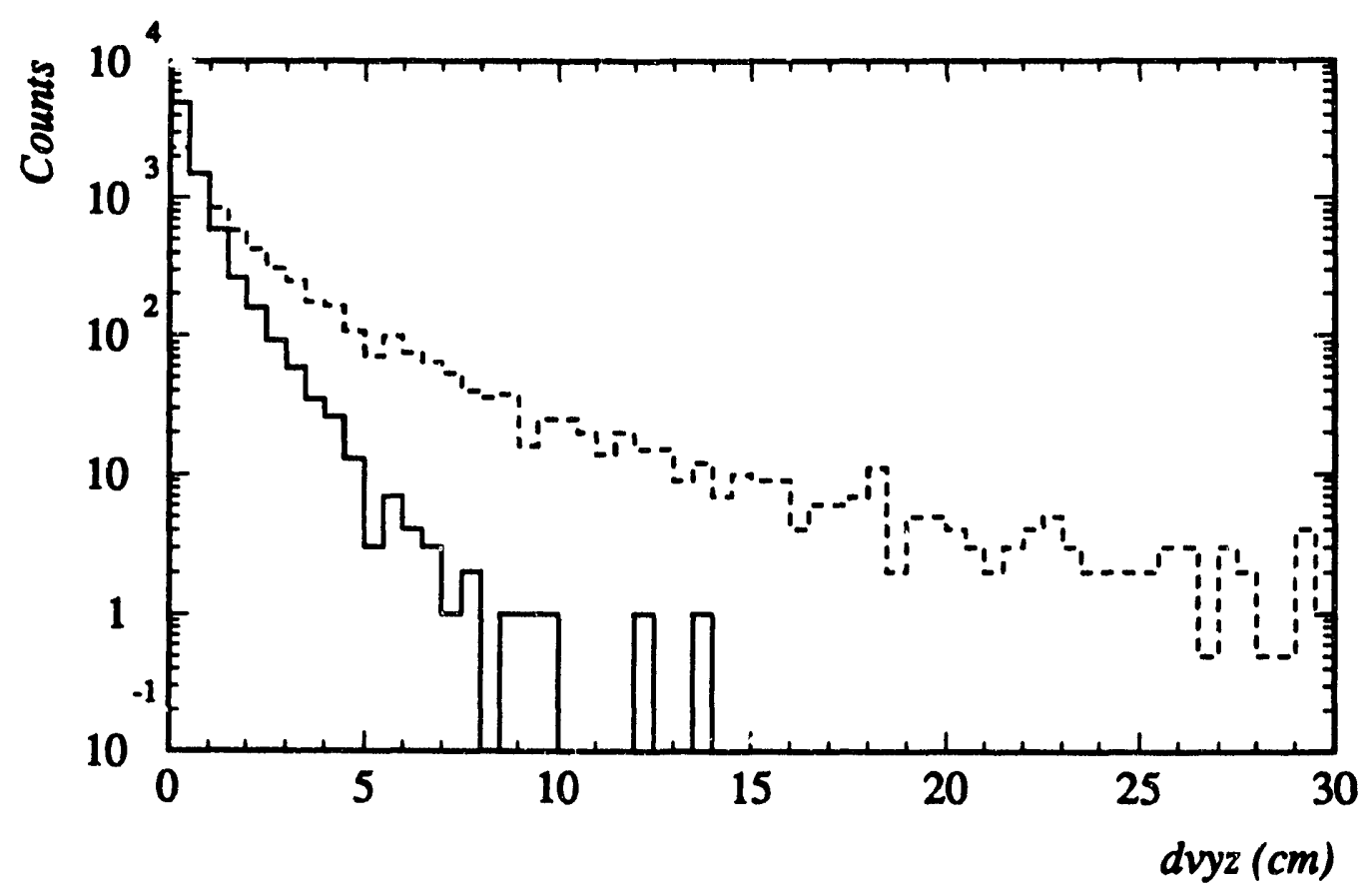

Figure 4.17: Projected distance to the start point. The data in the solid histogram have been reconstructed in three views, the data in the dashed histogram in two. The input is identical except for the suppression of measurements in one view.

distance of the start point from the vertex for simulated data. The tracks in the two-view and three-view histograms are identical, except for tracks which fail to reconstruct in two views because of bad stereo, accounting for $\sim 4 \%$ difference in the normalization. The two-view sample is obtained by ignoring the simulated measurements in one view. The semi-log plot emphasizes the difference in the tails. For two-view data, the projected distance $d v y z=\sqrt{d y^{2}+d z^{2}}$ is dominated by $d z$. The broadness of the distribution is surprising, even for three-view reconstructions, especially since non-vertex tracks have been omitted from the optical simulation. This feature has two origins: the start point is poorly constrained when the first detected point is far from the vertex or when bad stereo makes it difficult to determine track depth in the chamber. Such a broad distribution for vertex tracks presages the difficulty in making a clean cut to reject non-vertex tracks.

The limits imposed on the acceptance by the central cone and its influence on detection efficiency can be illustrated with simulated data. Figure 4.18 compares the rapidity density of negative hadrons $d N / d y$ as a function of rapidity $y$ of FRITIOF Monte Carlo events before and after the cone cut. The solid line is the input FRITIOF distribution. The dashed line is comprised of tracks which satisfy splmax $>20 \mathrm{~cm}$ in three camera views, i.e., of tracks in the three-view acceptance. The simulation is cutoff at $y=4.5$, about one unit of rapidity higher than the edge of our acceptance. For a fixed-target experiment at incident momentum $p_{\text {beam }}=$ $200 \mathrm{~A} \mathrm{GeV} / c$ the beam rapidity is $y_{\text {beam }}=6.0$, the target rapidity $y_{\text {target }}=0$. The distribution is normalized by the number of events so the integral over the full phase 


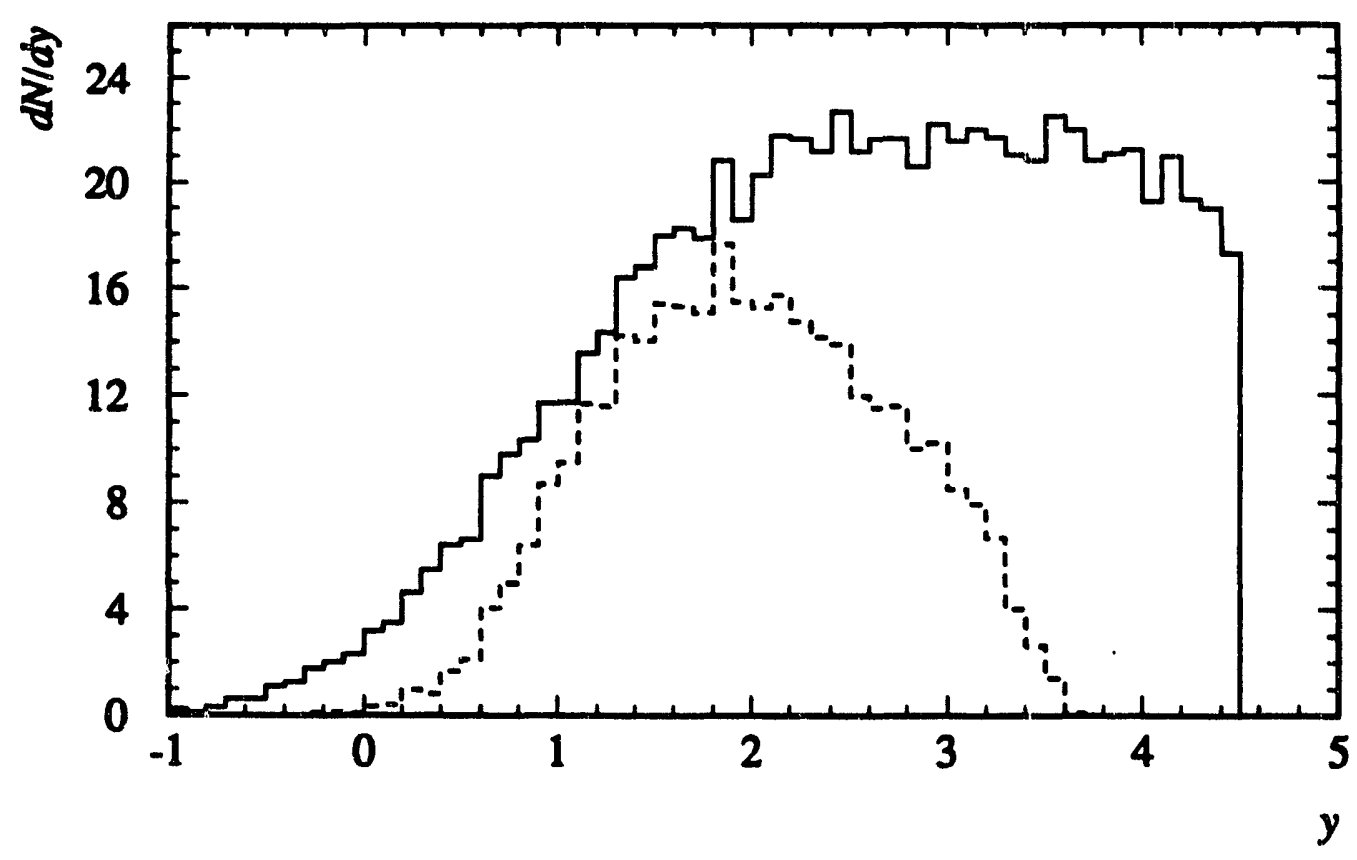

Figure 4.18: Normalized rapidity density of negative hadrons. The solid line is the FRITIOF distribution. The dashed line is the subset of tracks which have sufficient projected arc length in each camera image to be reconstructed in three views.

space yields the average multiplicity of produced particles. Note the central plateau about midrapidity characteristic of inclusive particle production in string models of hadron-hadron collisions. Note also the sharp fall-off in detection efficiency above $y \simeq 2$. The impact of the cone is also evident in the $\psi$ distribution of Figure 4.19, the azimuthal angular distribution in the transverse momentum plane (see Figures 4.3 and 4.4). A track with dip $\lambda=0$ pointing towards the cameras would have $\psi=0$, one with $\lambda=0$ pointing away from the cameras would have $\psi=$ $\pi$. Because of azimuthal symmetry about the beam axis the $\psi$ distribution should be flat. This is indicated by the dotted line in the figure for charged particles before the cone cut. The solid(dashed) line is for positively(negatively) charged particles after the cone cut has been applied. The rightside for positively charged particles covers the $\psi$ domain $0<\psi<\pi$. Except for the overall yield, the distributions for positively and negatively charged particles are reflection-symmetric about $\psi=\pi$, in particular with respect to the camera holes indicated by arrows in the figure. Tracks which point directly towards or away from a camera image as points and cannot be detected. There is a strong rightside-wrongside asymmetry for both histograms. Note the absence of any clear plateau in $\psi$ signalling a bias-free region. For this reason we have employed a procedure which $\psi$ symmetrizes the reconstructed momenta on a track-by-track basis for the calculation of geometrical weights. If the measuring, matching and reconstruction efficiencies were $100 \%$, the measured distribution of Figure 4.18 could be exactly inverted by the geometrical weights to obtain the true rapidity distribution for $0.4<y<3.0$. Because of 


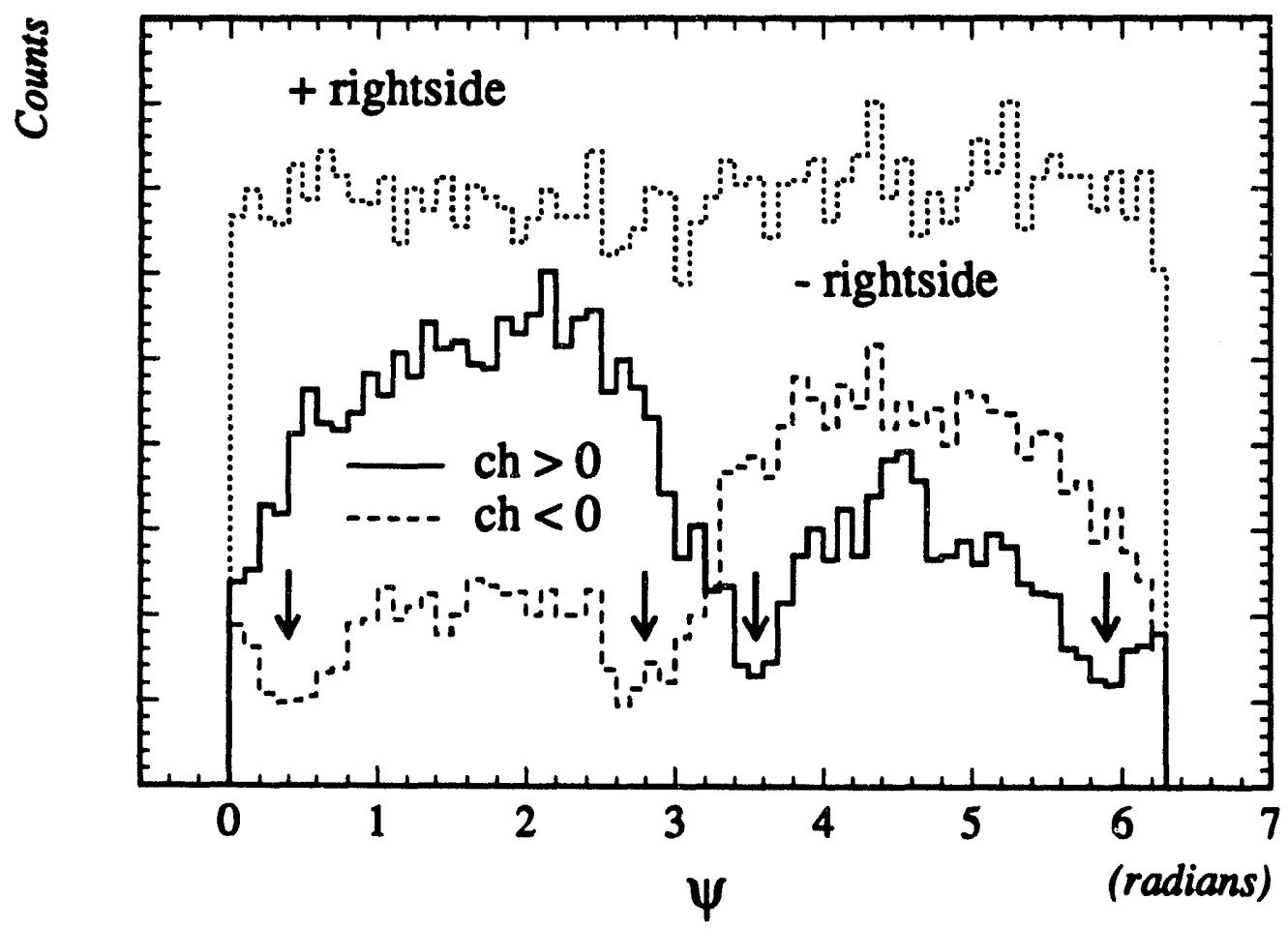

Figure 4.19: $\psi$ distribution for positively and negatively charged particles. The solid(dashed) line is for positively(negatively) charged particles. The dotted line is the input distribution, which is flat except for statistical fluctuations. Arrows mark the camera holes. Note the strong rightside-wrongside asymmetry in yield. 
the reflection symmetry about the center-of-mass in $A A$ collisions of equal-mass nuclei, this measurement would cover nearly the full phase space.

\subsection{Track Matching}

The association of track images in different camera views with the same space curve takes place in three stages. An initial set of track candidates is selected by demanding optical consistency between track images in different views. Optical consistency is defined by constructing projective envelopes for a track image from the camera-to-camera mappings $C$ and $C^{\prime}$ introduced in Section 4.2. This set is reduced by submitting the candidates to online reconstruction. The final set of matched tracks is selected by performing ambiguity resolution on the subset of optically consistent candidates which successfully reconstruct. Completely unassisted computer track matching must address two performance issues. First, what is the efficiency for the matching and reconstruction procedure and is there any bias associated with the loss of tracks? Second, how powerful is the procedure at the rejection of spurious tracks?

Completely unassisted computer matching has an intrinsic limitation compared to conventional matching-it will only be as good as the initial track measurement. There is a symbiotic interplay between measuring, matching and momentum reconstruction, especially if the scanner is aided by software tools which help assess the correctness of results. The prediction of end points and possible track directions, or the prediction of track images from two-view reconstruction inform the scanner where to look and necessarily improve scanning efficiency. However, if two-view reconstructions are included to compensate for tracking or scanning inefficiency, the efficiency of computer matching can approach that of conventional matching, with a potentially dramatic increase in the data reduction rate. Moreover, given a reasonable model for the track measuring, both the efficiency and the spurious track rejection power can be quantitatively estimated from simulation.

The method outlined above is first applied with the proviso that only threeview candidates be accepted. After ambiguity resolution for the three-view set, the unassociated tracks which remain are reprocessed in a second pass which explicitly searches for two-view candidates. Approximately $80 \%$ of the data successfully reconstruct and match in three views.

\subsubsection{Projective Envelopes}

Optical consistency is an absolute prerequisite for a correct track match. The projective envelope candidate selection procedure consists of two complementary tests, ${ }^{11}$ a chamber-boundary envelope test based on mapping $C$, and a dip planeboundary test based on mapping $C^{\prime}$. See Figure 4.5.

\footnotetext{
${ }^{11}$ In this section $C$ and $C^{\prime}$ refer to the extended mappings from measurement space to measurement space rather than film plane to film plane.
} 
The chamber-boundary envelope in view 2 of track $i$ from view 1 is the set of points in the measurement space of view 2

$$
\left\{\left(x_{2}, y_{2}\right) \mid\left(x_{2}, y_{2}\right)=C\left(x_{1}, y_{1}\right)\right\}
$$

for all $\left(x_{1}, y_{1}\right)$ belonging to the track. Recall that for each $\left(x_{1}, y_{1}\right),\left(x_{2}, y_{2}\right)$ is the set of points mapped from the single point $\left(x_{1}, y_{1}\right)$. It is constructed in practice by mapping a subset of measured track points, including the first and last. Each point maps to a line segment. The exterior boundary of the set of line segments approximates the envelope. A track $j$ in view 2 is optically consistent with track $i$ if $j$ is contained within the envelope. In most cases it is sufficient to calculate the intersection of track $j$, in circle approximation, with the line segments from track $i$ and require that each intersection occur between the endpoints of the corresponding segment. The calculation is illustrated in Figure 4.20. The upper figure is a typical normal envelope. The shaded region is the interior of the boundary of the set of line segments and in this case is a faithful representation of the envelope as defined by Equation 4.23. Of the ten tracks displayed in the figure only three, tracks 3, 24 and 54 drawn as dark solid lines, are consistent with the projective envelope, which hints at the combinatoric reduction power and selectiveness of the test. The remaining $\sim 80$ tracks measured in this view, which are even more remote from the envelope, have been omitted from the figure. Also shown in the figure are the three stereo axes for the $1 \Leftrightarrow 2,2 \Leftrightarrow 3$, and $3 \Leftrightarrow 1$ stereo systems. As discussed in Section 4.2 the line segments are approximately parallel to the stereo axis, in this example to the $1 \Leftrightarrow 2$ stereo axis. Note that the curves bounding the envelope are approximately parallel to the correct match, track 24-the boundary curves resemble trajectories.

The lower figure demonstrates a case in which the calculation might fail because of bad stereo. Note the apparent "bowtie" singularity where the boundary curves cross. It is impossible for the image of a point under $C$ to shrink to a single point. In this case the shaded region does not adequately represent Equation 4.23-the projective envelope is more complicated. When the track tangent $\vec{t}_{j}$ in view 2 is parallel to the stereo axis, the intersection of circle with line reduces to the point of tangency. The accumulation of numerical errors from the mapping $C$ and the circle fit can lead to a null intersection. Bad stereo points are flagged and omitted from the computation. Tracks with small radii of curvature in the bend plane always have bad stereo in at least one stereo system. These sharply curving tracks can also fail because of errors in the extrapolations of circle fits, especially if there are differences in measured arc length from view to view. In these cases the chamber-boundary envelope test is relaxed.

The dip plane-boundary envelope test ${ }^{12}$ is more stringent because it incorporates a physical constraint-the mapping $C^{\prime}$ focuses to a point as measurements approach the target (See Section 4.2). Construction of the envelope is based on a conformal mapping which transforms circles into lines [Han88]. A track in mea-

\footnotetext{
${ }^{12} \mathrm{M}$. Fuchs introduced us to the idea of restricting the projective envelopes by using the intersections of vision lines with the maximal dip planes discussed in Section 4.2.
} 


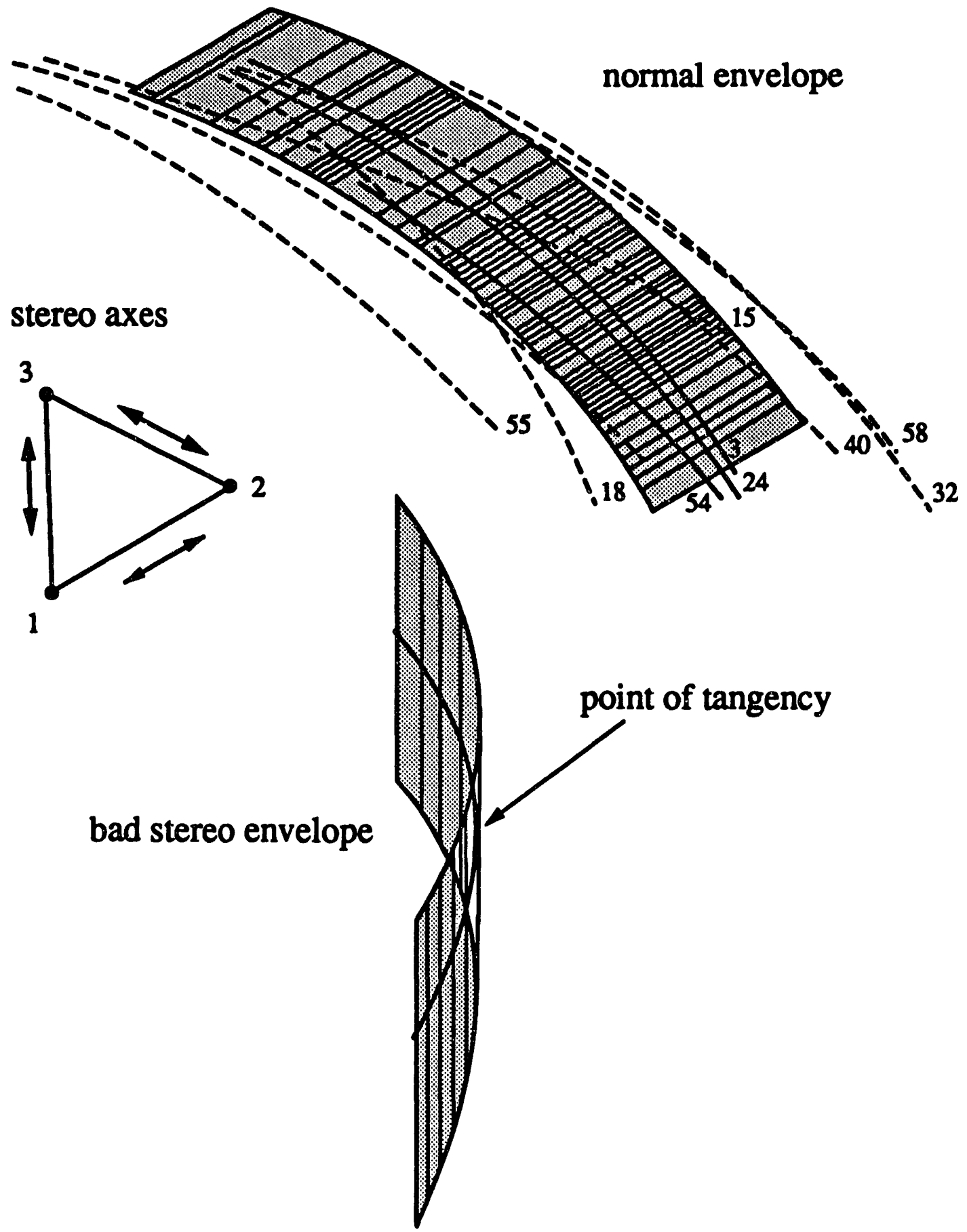

L

Figure 4.20: Chamber-boundary envelope. The envelope in the upper figure was constructed for the $1 \Leftrightarrow 2$ stereo system. The triangle, labeled by camera number, shows all three stereo axes. The bad stereo envelope in the lower figure was constructed for the $3 \Leftrightarrow 1$ stereo system. Note the point of tangency. The shaded region is an inadequate representation of Equation 4.23 for the case of bad stereo. 
surement space is represented by the circle

$$
(x-a)^{2}+(y-b)^{2}=r^{2} .
$$

If one assumes that all tracks emanate from a common vertex and translates to target coordinates so that $\left(x_{v t x}, y_{v t x}\right)=(0,0)$, then for all tracks

$$
a^{2}+b^{2}=r^{2}
$$

The mapping

$$
u=\frac{x}{x^{2}+y^{2}}, \quad v=\frac{y}{x^{2}+y^{2}}
$$

transforms circles into straight lines of the form

$$
v=\frac{1}{2 b}-u \frac{a}{b}
$$

Since conformal mappings preserve angles, the projected angle of emission $\theta_{\text {proj }}$ is unchanged-the slope of the line in $u v$ space equals the slope of the tangent vector to the track in measurement space at the assumed vertex. A transformation of this type is called an involution because points close to the origin in the domain space are mapped to distant points in the transformed space and vice-versa.

For a track $i$ in view 1 , vision lines are constructed for each measurement. The vision line intersections with the upper and lower maximal dip planes, mapped un$\operatorname{der} C^{\prime}$ to the measurement space of camera 2, are treated separately and regarded as pseudo-trajectories. Their projected angles of emission at the vertex are denoted by $\theta_{u p}$ and $\theta_{\text {low }}$, respectively. The pseudo-trajectories are transformed to straight lines in $u v$ space by Equations 4.24-4.27. The dip plane-boundary envelope is the quadrilateral in the $u v$ space associated with view 2 bounded by these straight lines and lines connecting their endpoints. It is displayed in Figure 4.21 along with the involutions of all tracks $j$ imaged in view 2. The two dense bundles of short line segments arise from short, close-lying tracks in measurement space. Note that the bounding straight lines converge to a point. A track $j$ in view 2 is optically consistent with track $i$ from view 1 if

$$
\begin{gathered}
\theta_{\text {up }}<\theta_{j}<\theta_{\text {low }} \text { or } \\
\theta_{\text {low }}<\theta_{j}<\theta_{\text {up }},
\end{gathered}
$$

and if the perpendicular distance from track $j$ in $u v$ space to the point of convergence is small. Only one track, drawn as a solid line in Figure 4.21, satisfies the angular test. The dashed segment indicates its extrapolation to the point of convergence. This test is robust against bad stereo failure-the point of convergence, $\theta_{\text {low }}$, and $\theta_{u p}$ are relatively insensitive to the direction of the stereo axis. It breaks down for sharply curving tracks, where the paths traversed by the vision line-dip plane intersections deviate from pseudo-trajectories and are mapped to curves rather than lines under the involution. As for the chamber-boundary envelope, the test is relaxed in this case to maximize efficiency. 


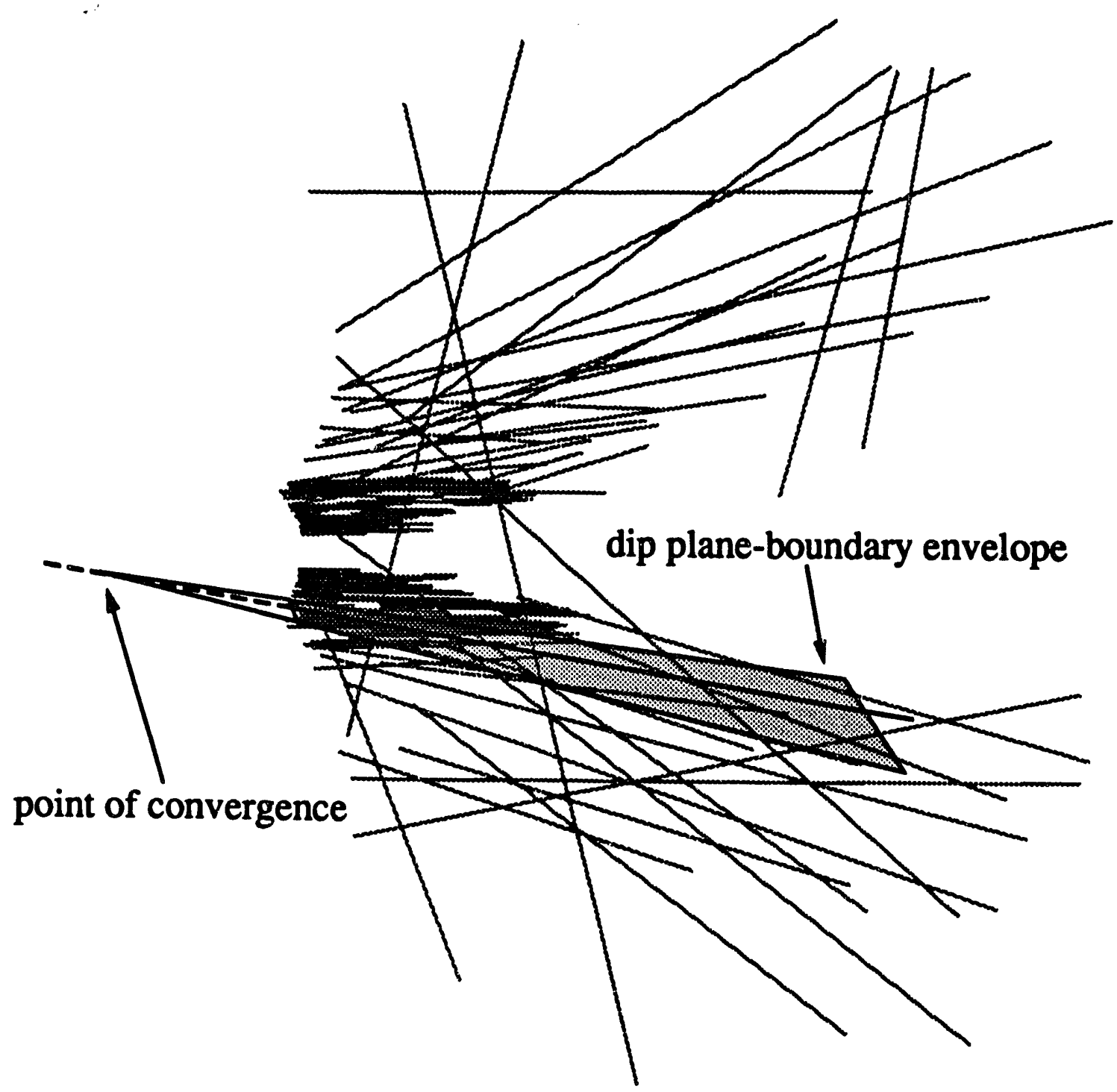

\section{L}

Figure 4.21: Dip plane-boundary projective envelope. The gray lines are the involutions of all tracks measured in view 2 . Only one track, indicated by a dark solid line, satisfies this test. The dashed line extrapolates the track to the point of convergence. 
The calculation described above with explicit reference to the $1 \rightarrow 2$ camerato-camera mappings is repeated for the camera pairs $2 \rightarrow 3$ and $3 \rightarrow 1$. Optically consistent pairs are compiled into lists. $T_{i j}^{1 \rightarrow 2}$ is the list of tracks, identified by a track number $j$ assigned during track measurement, in view 2 which are projectively consistent with track $i$ in view $1 . T_{j^{\prime} k^{\prime}}^{2} \overrightarrow{3}^{3}$ is the list of the $k^{\prime}$ tracks in view 3 which are projectively consistent with track $j^{\prime}$ in view 2 . Similarly for $T_{k^{\prime \prime} i^{\prime \prime}}^{3}$. For successful track candidates $(i, j, k)$ the three-way, cyclic comparison must yield $j=j^{\prime}, k^{\prime}=k^{\prime \prime}$, and $i^{\prime \prime}=i$. The second pass search for two-view matches starts with all successful track doubles $(i, j),\left(j^{\prime}, k^{\prime}\right),\left(k^{\prime \prime}, i^{\prime \prime}\right)$.

The projective envelope calculation was optimized to maximize efficiency and combinatoric reduction power. When both tests are combined, there is a large increase in combinatoric reduction with a negligible diminution in projective effciency. The projective efficiency for three-view candidates, calculated for simulated data, is $\mathbf{9 8 . 8 \%}$. It is necessarily higher for two-view candidates. If $n_{1}, n_{2}, n_{3}$ are the number of tracks measured in views 1,2 , and 3 , respectively, the combinatoric reduction can be defined by $n_{1} n_{2} n_{3} / n_{\text {cand }}$ where $n_{\text {cand }}$ is the number of candidates. By this measure the combinatoric reduction factor is typically $\sim 1000$. The average number of candidates submitted to online reconstruction was $\sim 600$, leading to an average CPU execution time for the complete track matching of 6 minutes/event on a $\mu$ VAX 3200 computer.

\subsubsection{Online Reconstruction}

Online reconstruction means momentum reconstruction which excludes information that must be deduced globally from an event as a whole, in particular, precise knowledge of the vertex. It is a geometrical fit rather than a particle fit because physical effects that depend on mass and charge-energy loss and multiple scattering-are neglected.

The track fit described in Section 4.2 is performed with $p(s)=p_{0}$, the constant momentum. The space-varying magnetic field $\vec{B}$ is included in the numerical integrations. Candidates which successfully reconstruct are subjected to the cuts enumerated in Section 4.5 plus the following additional test:

4. Points test. The points test identifies true three-view matches by demanding that nearly all measurements in all three views be used for the calculation of the residuals in the track fit.

The points test is crucial. For three-view candidates it suppresses disguised twoview reconstructions in which all measurements in one view have been rejected. For two-view candidates it inhibits skewed reconstructions which are dominated by measurements in a single view.

The efficiency of the online reconstruction is different for two-view and threeview reconstructions, primarily, as pointed out earlier, because of the higher probability of badstereo failure when only one stereo system is available. Approximately $5 \%$ of the simulated two-view tracks fail to reconstruct for this reason compared to 
about $1 \%$ of those measured in three views. The online reconstruction and track cuts reduce the number of candidates by factors of typically 4-8.

\subsubsection{Ambiguity Resolution}

After online reconstruction ambiguities generally remain-certain tracks in certain views occur in more than one candidate. Every track image is identified by a label, its index, a number between 1 and ntr, the number of tracks measured in a given view. The objective of ambiguity resolution is to find an optimal solution set which is mutually exclusive with respect to the labels. The correctness of the solution is predicated on two assumptions:

1. A correct match for each track image belongs to the candidate set.

2. The goodness-of-fit of the correct match, estimated by the reduced $\chi^{2}$ of the track fit, can not be significantly worse than that of an incorrect match with which it shares a label.

The second assumption is almost never violated. Because of scanning inefficiencies and because of small inefficiencies in the projective envelope test and online reconstruction the first assumption is sometimes false. This omission of a valid track, if accompanied by the acceptance of a spurious one with a shared label, may perturb the solution of an entire subset of tracks. The error propagates and may lead to the introduction of more than one spurious track. Ambiguity resolution is carried out separately for two- and three-view reconstructed candidates.

It begins with cyclic track rejection. Track candidate triples $(i, j, k)$ are sorted according to label, first with respect to the label $i$ in view 1 . If there exists more than one triple for any view 1 label, the ambiguous triples with respect to the label are ordered according to the reduced $\chi^{2}$ of the track fit. By virtue of assumptions 1 and 2, a candidate is rejected if

$$
\chi_{\text {cand }}^{2}>f \chi_{\min }^{2} .
$$

$\chi_{\min }^{2}$ is the minimum $\chi^{2}$ for the set. The numerical factor $f$ allows false triples to have somewhat better track fits than the correct match. It was tuned from studies of simulated data so that a correct triple is rejected less than one time in 1000 . The sorting, ordering and rejection procedure is repeated with respect to the labels $j$ and $k$ in views 2 and 3 respectively. Two-view candidates, which include track doubles with $(i, j, \emptyset),\left(\emptyset, j^{\prime}, k^{\prime}\right),\left(i^{\prime \prime}, \emptyset, k^{\prime \prime}\right)$ labels where the $\emptyset$ symbol indicates the omission of a partner in the respective view, are treated as one sei. If $i=i^{\prime \prime}$, for example, the doubles $(i, j, \emptyset)$ and $\left(i^{\prime \prime}, \emptyset, k^{\prime \prime}\right)$ will be sorted into the same subset for cyclic rejection. Cyclic track rejection reduces the scale of the ambiguity problem and increases the number of unambiguous track candidates.

The remaining track candidates are divided into ambiguity classes. An ambiguity class is a set of track candidates with at least one shared label between any two members. In principle the entire set of tracks entering ambiguity resolution can belong to a single ambiguity class. For each class, all possible solution sets 
mutually exclusive with respect to track labels are enumerated. Because of the requirement of mutually exclusive labeling, different enumerations within the same ambiguity class can have different numbers of members $n$. The optimal solution is defined as the enumeration which minimizes the estimator

$$
\delta=\frac{\sum_{i=1}^{n} \chi_{i}^{2}}{n^{\alpha}}, \quad \text { for } \quad \alpha=2,
$$

where $\chi_{i}^{2}$ is the reduced $\chi^{2}$ of the track fit of each unique member in the enumeration. The power $\alpha$ of $n$ in the denominator must be greater than 1 to favor enumerations with more members rather than ones with the lowest average $\chi^{2}$. The estimator $\delta$ has the desirable property that it depends strongly on $n$ for decisions between small solution sets and more strongly on overall goodness-of-fit for discriminating between large ones. It has a physical interpretation-the average $\chi^{2}$ per unit member.

The number of enumerations for evaluation can be large enough in exceptional cases to be impracticable on a minicomputer. In these cases the member of the ambiguity class with the largest $\chi^{2}$ is rejected iteratively until the number of enumerations is reduced to a manageable size. If a rejected candidate is a bridge candidate, one that connects two smaller classes into one large one, the scale of the calculation decreases rapidly. After resolution, candidates which have been rejected to limit the enumeration are restored if their track labels have failed to be associated with valid, matched tracks. The entire ambiguity resolution code is reapplied, with this rather smanl candidate set as its argument. Compared to the online reconstruction, the execution time for ambiguity resolution is negligible.

\subsubsection{Performance on Scanner Matched Data}

At an early stage in the development of the track matching a small set of reference CCD events were measured and matched by experienced film scanners in order to evaluate performance, identify problems, and guide subsequent refinements. ${ }^{13}$ At this time the projective envelope calculation was still several iterations away from its final form and the only cuts applied to track candidates were the points test and the goodness-of-fit cuts. An overall matching and reconstruction efficiency was defined as:

$$
\eta_{\text {match }}=\frac{\text { number of tracks reconstructed }}{\text { number of scanner matched tracks }} .
$$

The scanner matched set was compiled without any remeasuring and without any assistance from smart measuring tools-neither projections, nor end point predictions, nor online reconstruction. The reconstruction included the vertex fit as described in the next section, with a second track reconstruction that incorporated

\footnotetext{
${ }^{13}$ To obtain the reference set the scanners employed the conventional film technique. They had experience using this technique with film data taken at comparable track densities and multiplicities.
} 
the fitted vertex. It was initially assumed that all scanner triples were correct. The results of this exercise were:

$$
\eta_{\text {ocanner }}=94 \%, \quad n_{\text {matcher }}=93 \% \text {. }
$$

Departures from unity in the reference sample occur because of matching error, reconstruction inefficiencies, and the inclusion of nonvertex tracks which may be rejected after addition of the vertex. The same tracks of course fail the computer matching. The two methods agreed on the image association $96 \%$ of the time. A close examination of the discrepancies revealed that scanners sometimes measured triples which were optically impossible but still reconstructed successfully. These may have been cases where all the points in one view were rejected, but these compromised tracks were not flagged by the code. In certain instances it was possible to unambiguously decide that the scanner or the matching had been correct. After the encouraging performance, this comparison study was discontinued. It was too time consuming of scarce resources to gather meaningful statistics, impossible to arbitrate the disputes in certain cases, and ultimately it was difficult to tell who was calibrating whom. When online reconstruction is available as a measuring tool, ${ }^{14}$ in ambiguous cases scanners generally select the solution with the best goodness-of-fit. This suggests that the matching code evaluated scanner performance rather than the other way around.

The matching efficiency and spurious track rejection power, and whether they have combined to introduce a bias in a physical observable, will be discussed in Section 4.8. This discussion is deferred because the final reconstruction and vertex fit provide another layer of spurious track rejection.

\subsection{Final Reconstruction and Vertex Fit}

Matched data are submitted to the complete NA35 event reconstruction. The reconstruction code is built from application processors [Bru74] based on the HYDRA data management system [Boc81]. The small differences between the codes used for CCD-imaged and film-imaged data reflect the different optical and digitization environments. Because the CCD image sensor is fixed in space with respect to the streamer chamber, CCD-imaged data employ a single fiducial transformation $F$, whereas $F$ must be recalculated for each film frame. Because the CCD data were measured on a device with the same spatial resolution as the chip, the setting error is essentially the physical pixel size. Its precise value, $\sigma_{\text {meas }}=15 \mu \mathrm{m}$ in the film plane, was determined from the rms deviation of track fits in preliminary event reconstruction. See Figure 4.16.

The final track fit is a particle fit, performed for different mass hypotheses. Negatively charged particles are assumed to be pions. Positively charged particles are fitted with both the pion and proton mass. In most cases, because multiple

\footnotetext{
${ }^{14}$ The online reconstruction described in Section 4.6 was brought up for film-imaged data to aid the measurement at LBL of ${ }^{16} \mathrm{O}+{ }^{197} \mathrm{Au}$ data for an analysis of two-pion correlations. The statement in the main text is based on this experience.
} 
scattering and energy loss in the chamber are negligible, the fits are indistinguishable. The initial particle fits use the surveyed vertex position for the calculation of estimates to the track parameters but the fitted parameters are deduced from measurement residuals alone. After all particles have been reconstructed, the event vertex is calculnted by finding the space point which minimizes the sum of the squared distances of closest approach for all tracks in each event. The vertex fit uses the best mass fit for each track. It does not make use of the estimated vertex position. After the vertex has been determined, it is projected into the film plane and added as if it were a measured point to each imaged view, with a weight commensurate with the quality of the vertex fit. All tracks are refitted for all appropriate mass hypotheses. After the final track fit, the average distance of the start point $\left(x_{0}, y_{0}, z_{0}\right)$ from the vertex is $\simeq 100 \mu \mathrm{m}$. For $92 \%$ of the reconstructed tracks, this distance is $<300 \mu \mathrm{m}$. Because pions dominate the charged multiplicity, the particle fit for the pion mass hypothesis is selected for analysis.

The vertex fit is illustrated in Figure 4.22. $\overrightarrow{p_{0}}$ is the momentum vector at the start point $\overrightarrow{x_{0}}$. The distance vector from the hypothetical vertex to the start point

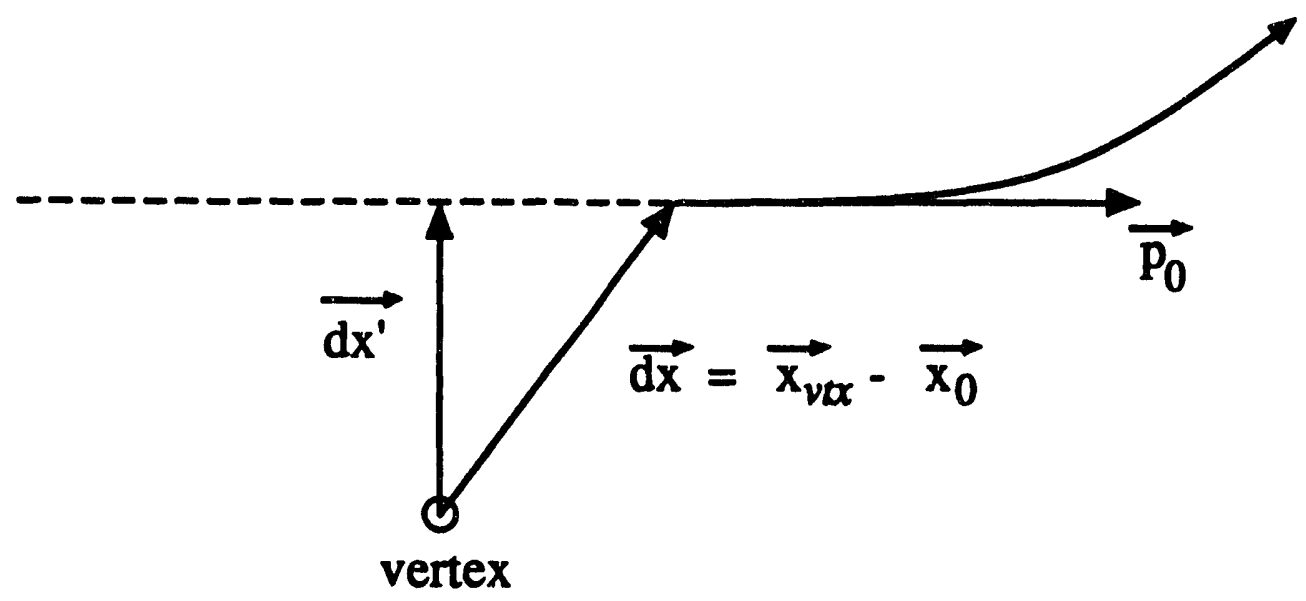

Figure 4.22: Geometry of the vertex fit. $\overrightarrow{p_{0}}$ is tangent to the bending track at the start point $\overrightarrow{x_{0}}$. The dashed line shows the extrapolation direction for the definition of the distance of closest approach $\left\|\overrightarrow{d x^{\prime}}\right\|$.

is $\overrightarrow{d x}=\vec{x}_{v t x}-\overrightarrow{x_{0}}$. Each track is linearly extrapolated along its momentum vector, modifying the start point until

$$
\overrightarrow{d x^{\prime}} \cdot \overrightarrow{p_{0}}=0
$$

Once $\overrightarrow{d x^{\prime}}$ has been determined, the contribution of each track to the overall $\chi^{2}$ of the vertex fit is computed:

$$
\chi_{j}^{2}=\sum_{k=1}^{3} \sum_{l=1}^{3} d x_{k}^{\prime} d x_{l}^{\prime} V_{k l}^{-1}
$$


$V$ is the $3 \times 3$ covariance matrix for the start point subspace of the track fit. It is obtained from the $2 \times 2$ covariance matrix in the local fit frame (see page 67) by a similarity transformation. $V$ is appropriately updated during the linear extrapolation. The vertex is found from a least-squares fit which minimizes

$$
\chi_{f i t}^{2}=\sum_{j=1}^{n} \chi_{j}^{2} .
$$

Once the vertex is known, $\chi_{j}^{2}$ is recalculated from Equation 4.32. The track with the largest $\chi_{j}^{2}$ is omitted and the vertex refitted if $\chi_{j}^{2}>\chi_{\max }^{2}$, a cutoff parameter. The procedure is iterated until $\chi_{j}^{2}<\chi_{\max }^{2}$ for all $j$ in a subset of the $n$ tracks in the event. In a typical event, $\sim 10 \%$ of the reconstructed tracks are omitted from the vertex fit. Large $\chi_{j}^{2}$ are expected because of the presence of non-vertex tracks from $\gamma$ conversion in the target, secondary interactions in the target and detector material, and the decay of neutral strange particles.

Figure 4.23 displays the results of the vertex fit for the 216 events in the complete data set. The upper figure shows the distribution of vertex coordinates $v x$ along the beam direction. The mean and $\sigma$ of the Gaussian fit are $-108.4 \mathrm{~cm}$ and $0.253 \mathrm{~cm}$, respectively. The surveyed vertex position at the center of the target was $(-108,0,0)$. The width of the distribution is consistent with the size of the .57 $\mathrm{cm}$ thick ${ }^{32} \mathrm{~S}$ target required to obtain a $1 \%$ interaction probability. Distributions for $v y$ and $v z$, the transverse coordinates, are shown together in the lower figure. Note the change in scale from $\mathrm{cm}$ to $\mathrm{mm}$. The mean and $\sigma$ of the Gaussian fits in the $y$ and $z$ directions are $(0.389,0.575) \mathrm{mm}$ and $(-2.256,1.354) \mathrm{mm}$, respectively. Both widths are consistent with an estimated beam spot size of $\simeq 1 \mathrm{~mm}$. The broadness of the $v z$ distribution is a consequence of the poorer out-of-plane position resolution due to projective geometry.

One would like to reject non-vertex tracks on the basis of probability. If the distances of closest approach are normally distributed the $\chi_{j}^{2}$ statistic of Equation 4.32 should be distributed according to the $\chi^{2}$ probability density function

$$
\begin{aligned}
f\left(\chi^{2}, \nu\right)= & \frac{1}{2^{\nu / 2} \Gamma(\nu / 2)} \chi^{2[(\nu / 2)-1]} \exp \left(-\chi^{2} / 2\right) \\
& \longrightarrow 1 / 2 \exp \left(-\chi^{2} / 2\right)
\end{aligned}
$$

for $\nu=2$ degrees of freedom. The two degrees of freedom correspond to the two independent coordinates of the start point. This is explicitly demonstrated in Figure 4.24 which plots the $\chi_{v t x}^{2}=\chi_{j}^{2}$ distribution for reconstructed, simulated track data. The simulated data are from the three-view acceptance reference sample, extended to include the average multiple scattering in the target and chamber entrance window. No tracks are lost because of scanning inefficiency and there are no matching errors. All tracks emanate from the canonical vertex position $(-108,0,0)$. The histogram has been normalized to unity so that it can be cumpared to the normalized probability density function, shown by the dashed line in the figure. It is not a fit. The associated probability distribution (not shown) for 

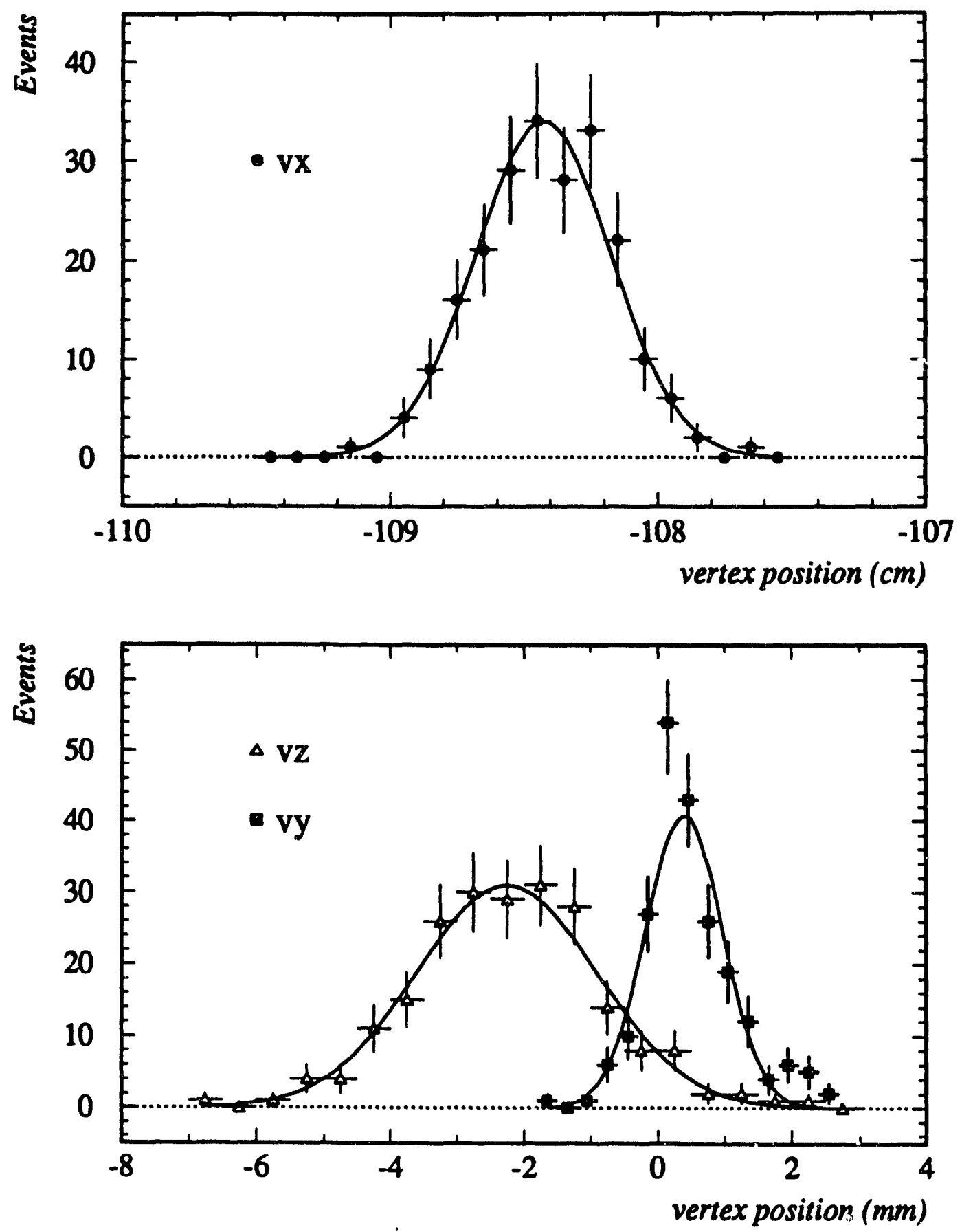

Figure 4.23: Vertex fit distributions. $x$ is the beam direction, $y$ and $z$ the transverse directions. The surveyed vertex position was $(-108,0,0)$. The beam spot at the SPS in fixed-target mode is $\simeq 1 \mathrm{~mm}$ in diameter. 


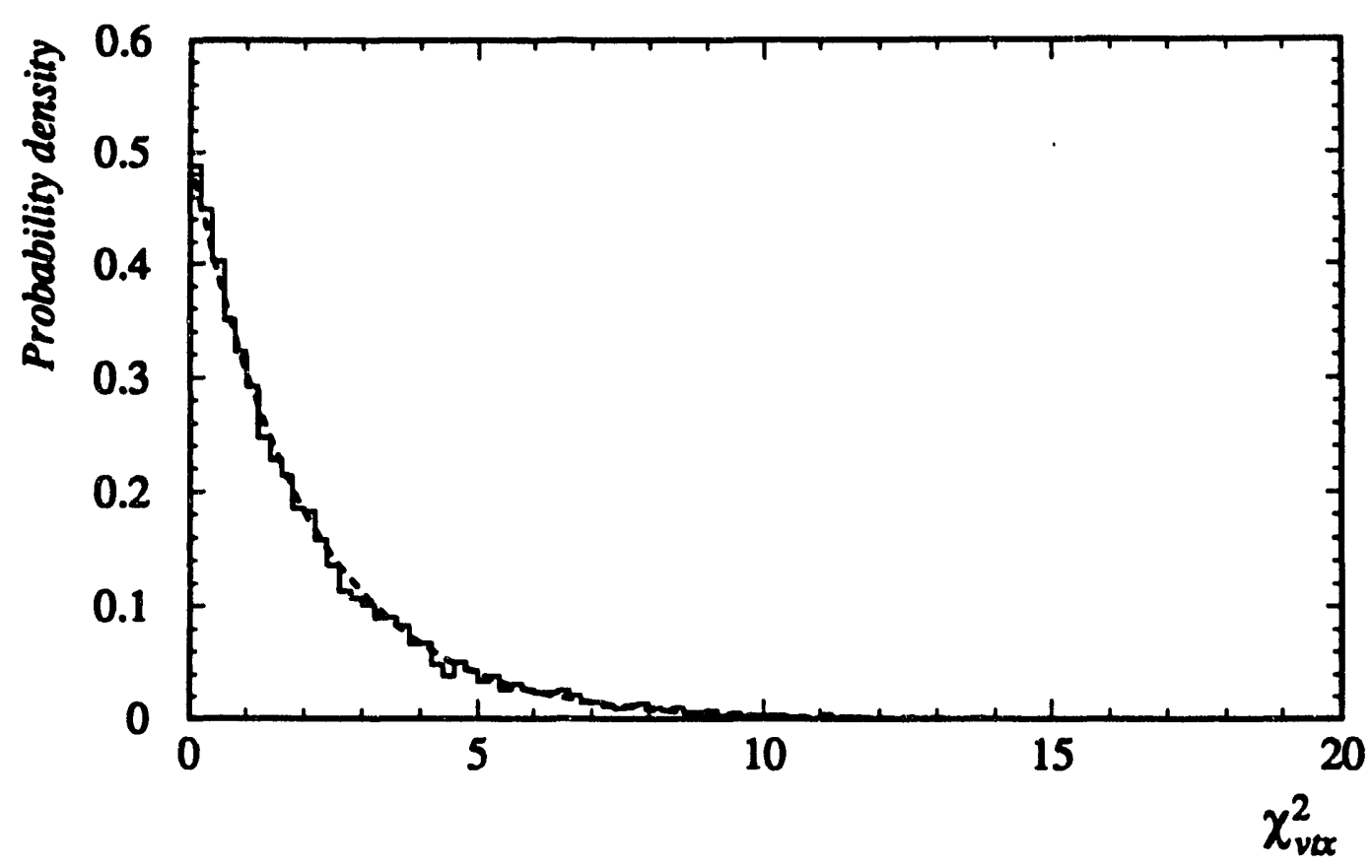

Figure 4.24: $\chi_{v t x}^{2}$ distribution for reconstructed simulated tracks with respect to the fitted vertex. The simulated data have been normalized to unit integral to compare with the plotted probability density function (dashed line).

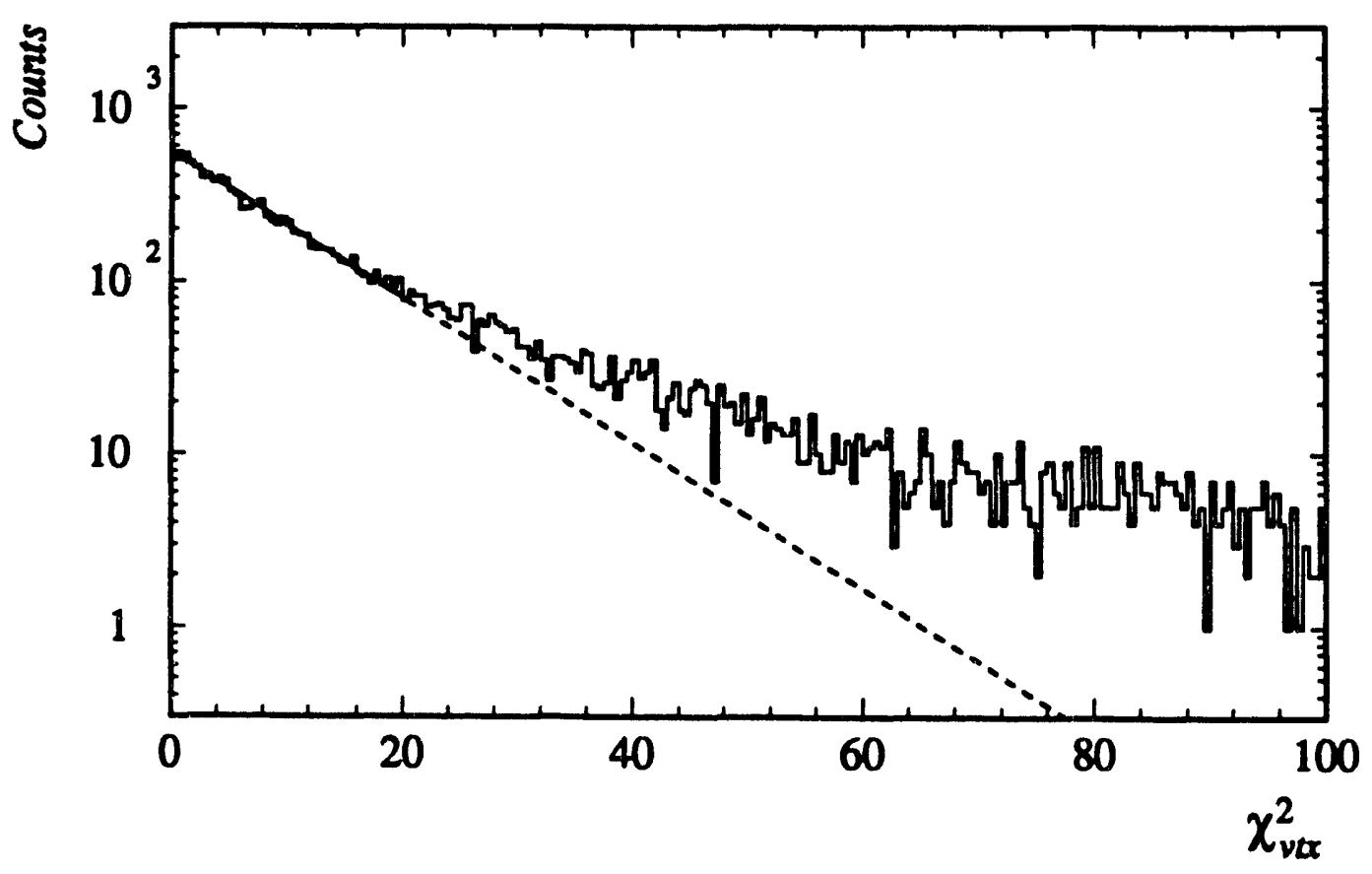

Figure 4.25: $\chi_{v t x}^{2}$ distribution for measured data. The data have been fit to an exponential out to $\chi_{v t x}^{2}=20$. The dashed line is a linear extrapolation of the fit beyond this exponential core. 
$P\left(\chi^{2}, \nu\right)$, obtained for each $\chi^{2}$ by integrating the density function from $\chi^{2} \rightarrow \infty$, is flat for all $P>.001$.

A similar distribution is plotted logarithmically in Figure 4.25 for the threeview subset of the measured data. Note the difference in scale between $\chi_{v t x}^{2}$ values for simulated data and measured data. The solid line is an exponential fit out to $\chi_{v t x}^{2}=20$, the region of the distribution we refer to as the exponential core. The fit has been extrapolated to higher values. $\chi_{v t x}^{2}=100$ was the cutoff parameter for track rejection and iteration during the vertex fit. The logarithmic plot emphasizes the non-exponential tail. Omitted from the histogram is a long tail out to extreme values. Approximately $10 \%$ of the reconstructed particles belong to this tail with $\chi_{v t x}^{2}>100$. From the difference in the integrated contents between the measured data and exponential fit, one can infer the contamination of the sample by nonvertex tracks. Including data in the long tail $\sim 18 \%$ of all reconstructed particles are most probably non-vertex or spurious tracks. In Chapter 5 we investigate the effect of non-vertex tracks on charged particle spectra and define a cut intended to suppress them.

The final track reconstruction and vertex fit improve the spurious track rejection power. Spurious tracks which succeed in online reconstruction sometimes fail after inclusion of the vertex. Even if their track fits succeed, they tend to larger values of $\chi_{v t x}^{2}$ and are more likely to be rejected by a $\chi_{v t x}^{2}$ cut.

\subsection{Matching Efficiency and Spurious Track Rejection}

It is difficult to estimate matching efficiency directly from measured data. Typically, a sample of events is independently measured in two laboratories and the number of accepted tracks is compared. For example, the double streamer chamber experiment at the ISR [Tho77] processed $\simeq 500$ events in this manner and found better than $98 \%$ agreement. However, this type of comparison is seldom made on a track-by-track basis, and the estimation of efficiency is relative rather than absolute. Moreover, a class of measured track images which fail to be successfully associated into matched and reconstructed tracks should be excluded from an effciency analysis. For example, nonvertex tracks and spiralling electrons constitute contaminations rather than signal, and not all tracks measured are imageable in all three views. Finally, shortcomings in the measuring induce reconstruction failure. In an operator-assisted approach tracks can be remeasured until the reconstruction succeeds although this may lead to biases in the momentum measurement that are difficult to estimate statistically. This approach was adopted for the measurement of $\pi^{-}$spectra from CCD data taken for ${ }^{197} \mathrm{Au}+{ }^{197} \mathrm{Au}$ collisions at the BEVALAC [Cha92] at $p_{l a b} \simeq 1 A \mathrm{GeV} / c$, where the relatively low abundance of produced particles made it practicable to perform the matching and reconstruction for full events while they were being measured. Since reconstruction is part of our matching, any reconstruction failures show up as matching inefficiencies. Rather than try to separate these effects, we will state a matching efficiency that includes 
these losses.

The analysis presented here is based on a track-by-track comparison made for simulated data. For this comparison, and for the extraction of charged particle spectra later, the three-view acceptance was defined by requiring splmax $>20 \mathrm{~cm}$ with respect to the analysis cone in all three views. Recall that tracks were measured if splmax $>15 \mathrm{~cm}$ in any view. This more stringent requirement improved efficiency without cutting into the acceptance and suppressed inconsistencies due to the discretionary interpretation of the measuring guideline for visible arc length (see Figure 4.11). The optimal solution for each event was the reference sample, the set of tracks in the three-view acceptance. A sample of 200 Monte Carlo events was created by following the complete simulation procedure folding in the effects of measuring error, obscuration, and the two-partition scanning efficiency. Note that the best achievable solution could contain less tracks than the reference sample if a track was lost to scanning inefficiency in more than one view. In this sense the efficiency estimate is absolute. All particles were emitted from a single vertex. An identification label was assigned to each imageable track in each view, in one-to-one correspondence with a track identifier in the parent event. This sample was processed through the complete data reduction chain-matching, final reconstruction and vertex fit, and an acceptance test. The acceptance test consisted of propagating particles through the NA35 magnetic field using the event-by-event fitted vertex and the reconstructed momenta as initial conditions, projecting the space trajectories into the CCD measurement space, and making the same three-view splmax cut used to define the reference sample. The acceptance test is described in more detail in Section 5.2. Each processing step provides another layer of spurious track rejection power.

Track matches were classified as correct if the associated images possessed the same parent labels, either two or three labels depending on whether the successful match was for two or three views. Tracks were denoted as compromised if three views were associated two of which had identical labels. Spurious tracks, either two- or three-view, possessed no common label. Spurious tracks could approximate correct matches if they were composed of tracks in the three-view acceptance with nearly the same momentum or they could be totally fictitious, composed of two or three images from tracks lying outside of the good acceptance. As the analysis of the small sample of hand matched data has shown, an admixture of compromised and spurious tracks is unavoidable in high multiplicity streamer chamber work. We know of no other attempt to quantify the effect. A final category of tracks, omitted tracks, was devised to account for tracks in the reference sample none of whose labels were used in any image associations. Depending on their phase space distribution, the absence of the omitted tracks could bias a physical observable as well as affect its overall normalization.

In order to define the efficiency, correct and compromised tracks are lumped together as valid kinematic solutions. The justification for this is shown in Figure 4.26 , which presents histograms of the differences in transverse momentum $p_{T}$ and rapidity $y$ between the ideal value of the respective kinematic variable for the parent track and the value calculated from the final momentum reconstruction 

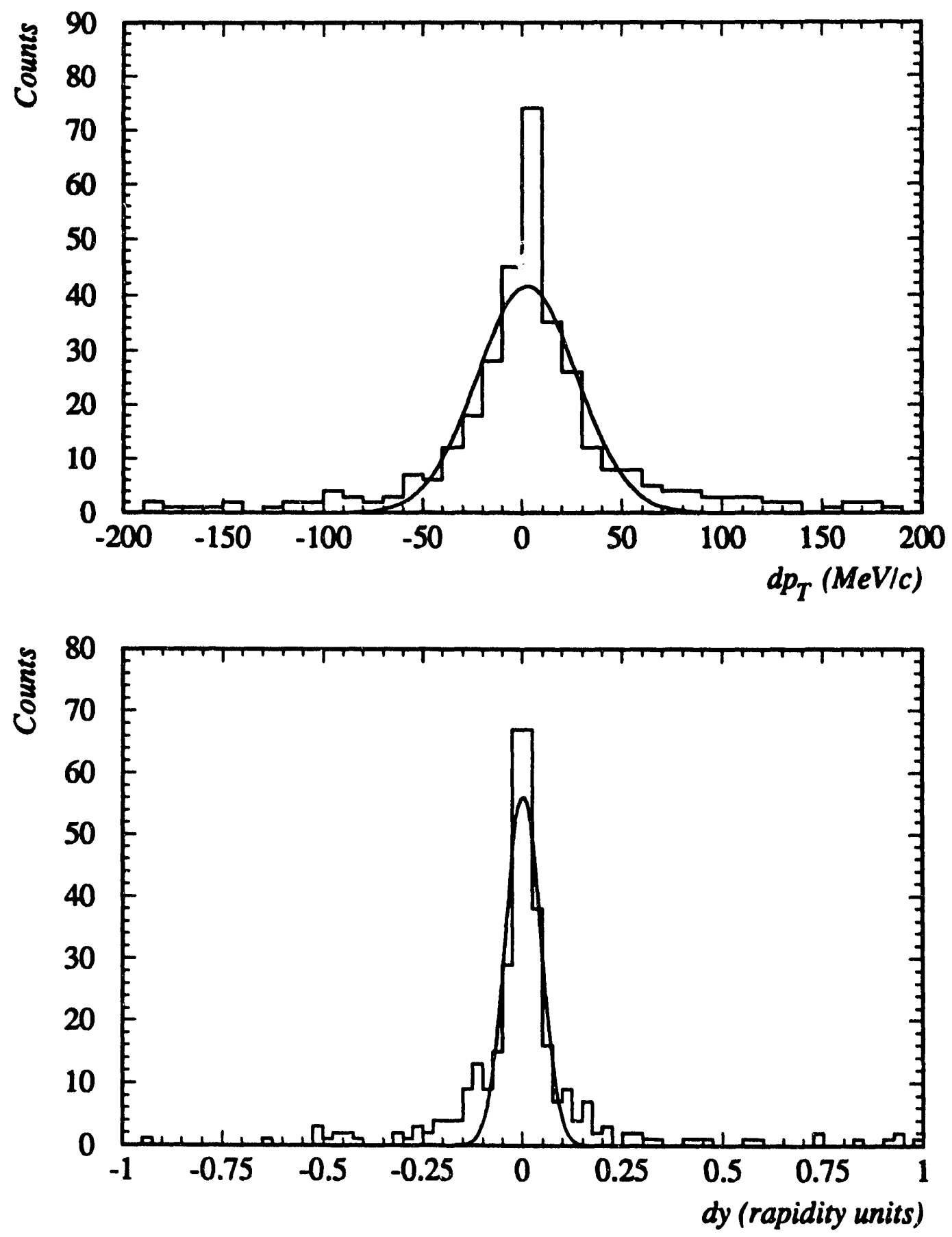

Figure 4.26: Differences in transverse momentum $p_{T}$ and rapidity $y$ for compromised tracks. Since the reference values have been taken directly from the input to the simulation and the compromised values have been determined from the reconstruction, the effects of momentum resolution are implicitly folded in. 
for the compromised track. The rapidity is calculated assuming the pion mass. The plots are strongly peaked near zero. The Gaussian fits for $p_{T}$ and $y$ have $\sigma_{p_{T}}=25 \mathrm{MeV} / c$ and $\sigma_{y}=0.046$ units of rapidity, respectively. Approximately $2 / 3$ of the compromised tracks have added an image from outside the three-view acceptance. The remainder have appropriated an image from a second three-view acceptance track. Both cases have difference distributions peaked near zero with approximately the same width, so they have been included together in Figure 4.26. According to the simulation study, the compromised tracks comprise $2.5 \%$ of the yield in the three-view acceptance. As discussed below, a reasonable $\chi_{v t x}^{2}$ cut suppresses this contribution by more than a factor of two. Given the extent to which they "remember" the kinematic variables of a correct image association and their small yield, the compromised tracks are unlikely to distort single particle inclusive spectra.

For each event the matching efficiency $\eta_{\text {match }}$ is defined from the number of correct and compromised tracks, $n_{\text {corr }}$ and $n_{\text {comp }}$, and from the maximum possible number of reconstructed tracks in the three-view acceptance reference sample $n_{3 V}$,

$$
\eta_{\text {match }}=\frac{n_{\text {corr }}+n_{\text {comp }}}{n_{3 V}} .
$$

The distribution of $\eta_{\text {match }}$ for the simulation study is shown in Figure 4.27. The

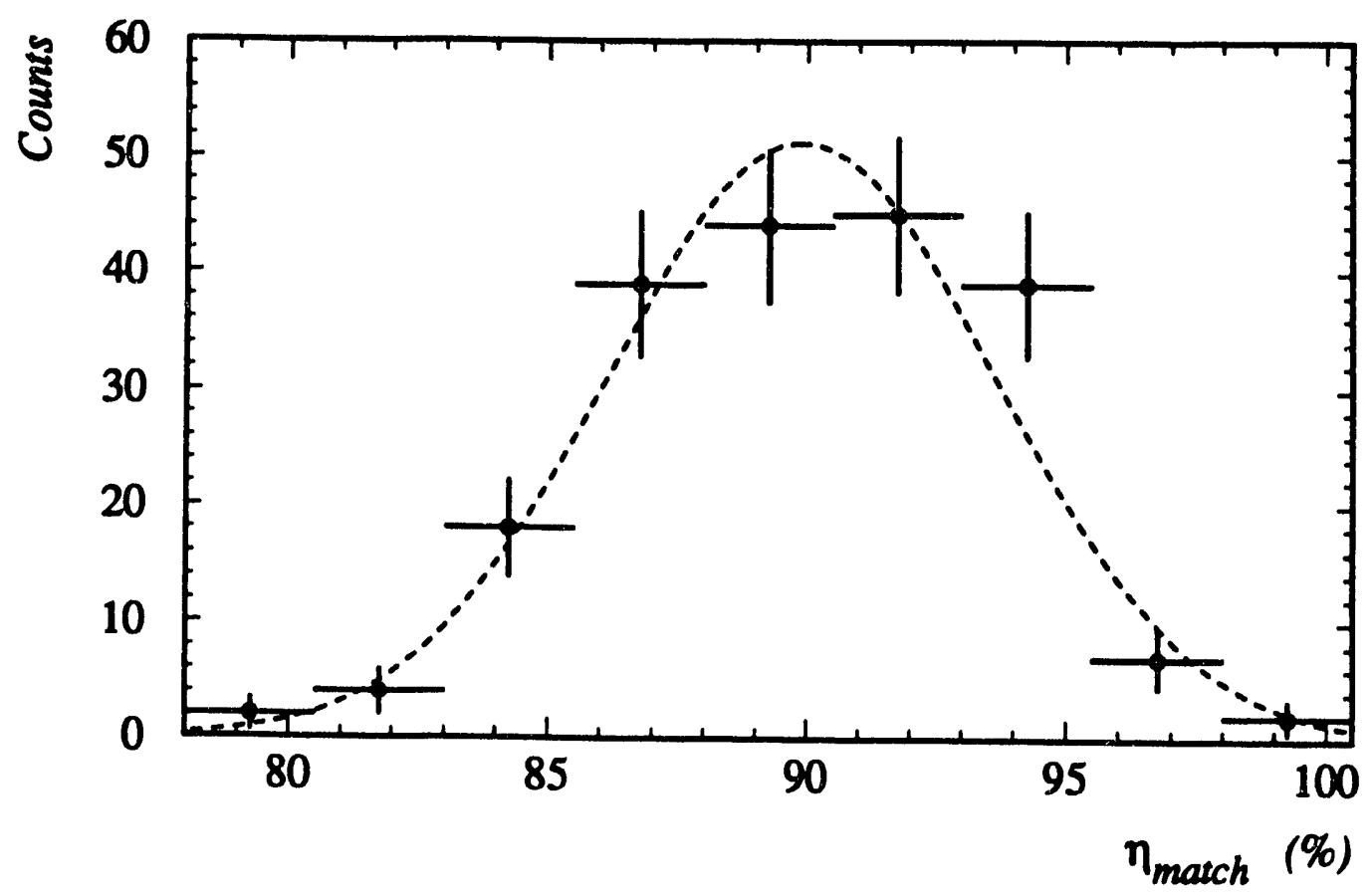

Figure 4.27: Matching efficiency $\eta_{\text {match }}$.

average efficiency is $\left\langle\eta_{\text {match }}\right\rangle=90 \%$ with $\sigma_{\eta}=3.8 \%$. The average reconstructed multiplicity in the three-view acceptance, which includes the spurious tracks $n_{a p}$ as well as $n_{\text {corr }}$ and $n_{\text {comp }}$, is $n_{\text {rec }}=73.53$. To first order, the efficiency is independent of multiplicity. If only three-view matches and reconstructions were accepted the 
average efficiency would fall to $78 \%$. This was the reason for extending the data reduction to include two-view matches.

A measure of the spurious track rejection power is the percentage of spurious tracks $\eta_{\text {opur }}$ which contaminate the data sample,

$$
\eta_{\text {opur }}=\frac{n_{\text {sp }}}{n_{\text {rec }}}
$$

$\eta_{\text {opur }}$ is plotted in Figure 4.28. The mean value is $\left\langle\eta_{\text {opur }}\right\rangle=5.8 \%$. The de-

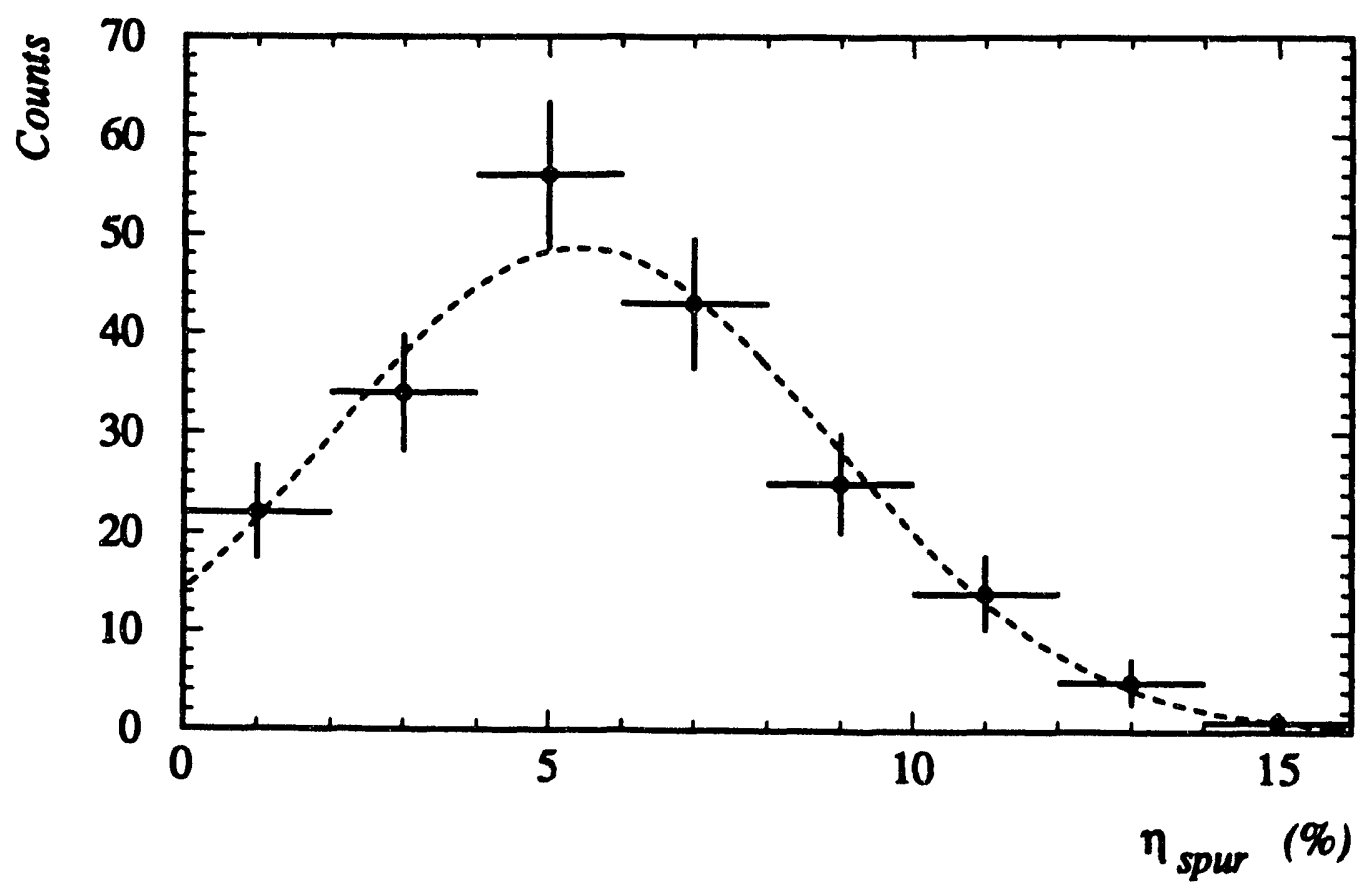

Figure 4.28: Spurious track contamination $\eta_{\text {opur }}$.

pendence of the spurious track contamination on multiplicity is also weak. Nearly $80 \%$ of the spurious tracks are two-view matches and reconstructions. There is less than one three-view spurious track per event. Combined with results for simulated data which have $100 \%$ scanning efficiency, for which spurious tracks virtually never occur and compromised tracks are rare, the analysis of the matching efficiency indicates that most of the deficiencies in the matching result from scanning inefficiencies induced by limits in optical resolution at high track density.

Most of the spurious tracks contain at least one image from a three-view acceptance track. To a limited extent, these preserve the kinematic values of a parent, however the difference distributions are rather broad and shifted away from zero. On average, $p_{T}$ is shifted toward higher values while $y$ is shifted toward lower values. This is demonstrated explicitly for the rapidity $y$ in Figure 4.29 which plots the rapidity density $d N / d y$ as a function of $y$ for three cases: i) the correct and compromised tracks which comprise the valid solutions, ii) the spurious tracks with their true rapidities from the input to the simulation, and iii) the spurious tracks with rapidities calculated from the momenta returned by the complete data 


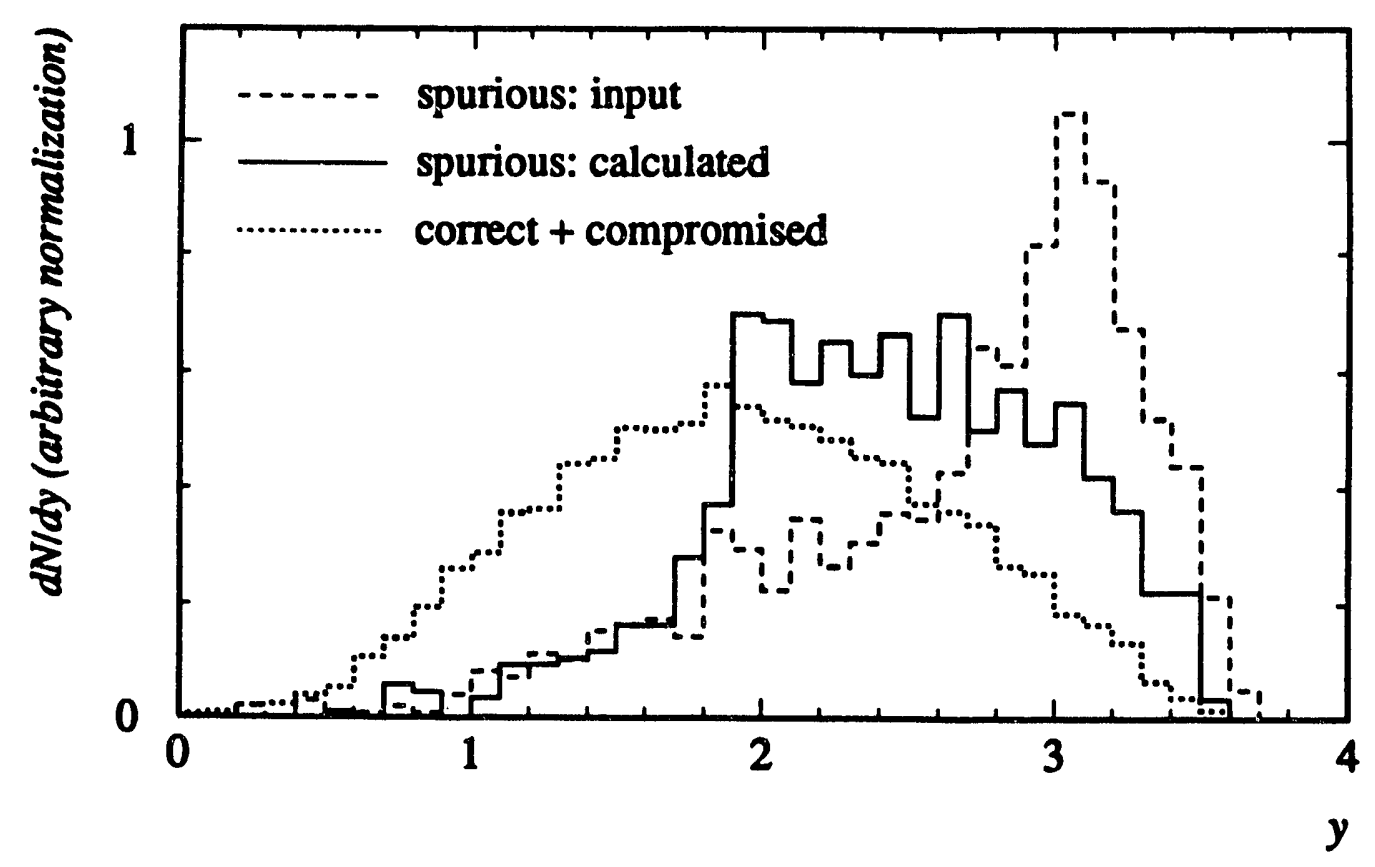

Figure 4.29: Comparison of the rapidity density $d N / d y$ for spurious tracks and correctly matched tracks. The spurious tracks are shown twice, once with the rapidity from the input to the simulation (dashed line), once with the rapidity determined from the reconstructed momenta (solid line). The pion mass is assumed in both cases. The relative normalization of the three histograms is arbitrary.

reduction procedure. For the comparison the three spectra, one of which (correct plus compromised) has a dramatically larger integrated value, have been separately normalized to unit integral $\int(d N / d y) d y=1$. The dashed(solid) curve is for the spurious tracks with the input(calculated) rapidity assuming the pion mass. The downward rapidity shift is clear. The dotted line is the distribution for correctly matched and reconstructed tracks. As would be expected, spurious tracks are comprised of images descending from parents near the edge of the rapidity acceptance. A similar effect is observed in the comparative $p_{T}$ distributions. We defer the conclusion on distortion to spectra to Chapter 5 until after the geometrical acceptance correction has been discussed. A rapidity cut at $y=2.8$, which is justified there, reduces the spurious track contamination by $\simeq 1 / 3$.

The omitted tracks, which have been excluded from Figure 4.29 to avoid confusion, essentially follow the distribution of the valid solution set, indicating their absence only affects the normalization. The mean value for the reference sample is $\left\langle n_{3 V}\right\rangle=76.93$. Compared to $\left\langle n_{\text {rec }}\right\rangle=73.53$ this results in an $\simeq 5 \%$ underestimation of the absolute normalization.

The figure for $\eta_{\text {spur }}$ quoted above is a somewhat conservative estimate because it was calculated without the benefit of a $\chi_{v t x}^{2}$ cut, although some spurious tracks fail to reconstruct after the addition of the vertex. Figure 4.30 shows the $\chi_{v t x}^{2}$ distribution for all matched and reconstructed tracks for the Monte Carlo data. Note the long tail, which was absent from the same distribution for the reference 


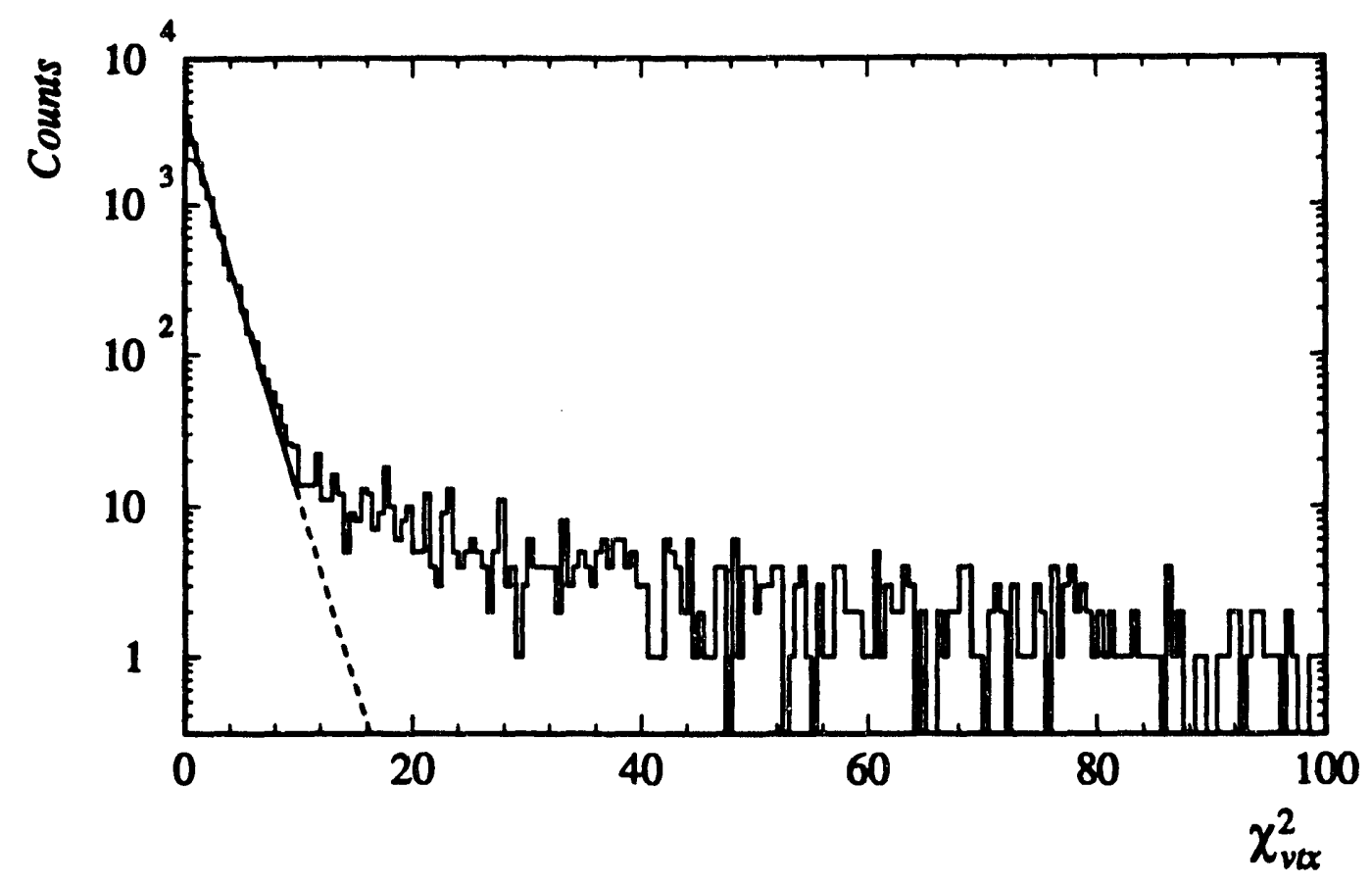

Figure 4.30: $\chi_{v t x}^{2}$ distribution for Monte Carlo data processed through the complete data reduction chain. The solid line is a fit to the exponential core. The dashed line extrapolates the fit. The tail is composed exclusively of spurious and compromised tracks.

sample plotted in Figure 4.24. The solid line is a fit to the exponential core. The dashed line extrapolates the fit. A cut demanding $\chi_{v t x}^{2}<20$ eliminates $\simeq 1 / 2$ of the spurious tracks, and nearly $2 / 3$ of the compromised ones. This cut rejects less than 1 valid track out of 1000 .

\subsection{Momentum Resolution}

The simulated data were also employed to judge the quality of the momentum information provided by the track fit. The two aspects investigated were the unbiasedness of the estimators of the track parameters, and the precision of the result, the momentum resolution. Although the critical measuring limitation for track matching is the scanning efficiency, the critical problem for momentum estimation is the obscuration. Because of it, the distance of the first measured point from the event vertex $s_{v t x}$ may be rather long. This has two consequences. First, the integration of the equations of motion during the track fit is not constrained by measurements for distances which may be as great as $120 \mathrm{~cm}$. Second, the fitted vertex, which may enter into the final track fit with a large weight, can exert a disproportionate influence on the final momentum. Both effects can introduce bias. $^{15}$

\footnotetext{
${ }^{15}$ In fact, difficulties encountered in the extrapolation of streamer chamber trajectories to a downstream RICH detector indicated a problem in the track fit. This was also evident in
} 
One method of testing fitted values for bias is to investigate the stretch functions or pulls of the fit parameters. The pulls are residuals, calculated between the observations and fitted values and normalized by the uncertainty in the residual [Fro79]. For a fitted parameter $x_{f}$ and a measured value $x_{m}$ with standard deviations $\sigma_{f}$ and $\sigma_{m}$ the pull quantity $z(x)$ is generally defined:

$$
z(x)=\frac{x_{f}-x_{m}}{\sqrt{\sigma_{f}^{2}-\sigma_{m}^{2}}} .
$$

The pull $z(x)$ is expected to have a distribution which is approximately the standard normal distribution $N\left(\mu=0, \sigma^{2}=1\right)$. A shift relative to zero demonstrates a bias and a width greater(less) than 1 indicates an error estimate that is too small(large).

By analogy we define pulls for the momentum fit parameters $1 / p, \lambda$, and $\phi$ taking the input values to the simulation as measured values which are exactly known:

$$
z(x=1 / p, \lambda, \phi)=\frac{x_{f}-x_{\text {oim }}}{\sigma_{f}} .
$$

The $\sigma_{f}$ are the respective diagonal elements in the covariance matrix of the track fit. The pulls, calculated separately for positively and negatively charged particles, are plotted in Figure 4.31.

There is evidence for small biases in all fit parameters with a strong chargedependent effect for the azimuth $\phi$. The negative pulls for $1 / p$ indicate a tendency to overestimate the momentum, that is, to make the track more rigid. The pull for $1 / p$ is independent of $p$ and of $s_{v t x}$. A strong dependence on $s_{v t x}$ was eliminated by the improved integration achieved with the addition of the pseudo-points. $z_{1 / p}$ is excessively narrow, suggesting a consistent overestimation of the error in the reciprocal momentum. Both $\lambda$ and $\phi$ have widths $\sigma \simeq 1$ indicating reasonable error estimates from the fit. The positive pull for $\lambda$ may be evidence for an inconsistency in the overall geometrical alignment of the beam/chamber/magnetic field/optical system. In Ref. Fri73, a similar effect was attributed to an error in the beam direction. The charge-dependent bias in the azimuth $\phi$ actually has an equivalent effect on both positively and negatively charged particles. For rightside particles it is relatively straightforward to see that a positive(negative) pull indicates a shift in azimuth towards the beam for negative(positive) particles.

The small biases discussed above do not seriously degrade the momentum resolution. The canonical expression [Glu63] for the uncertainty in $p$ is

$$
\left(\frac{\delta p}{p}\right)^{2} \propto \frac{1}{L^{4} B^{2}} p^{2}+\text { multiple scattering, }
$$

systematic deviations of the measurement space predictive overlays, obtained from the fitted momenta by track swimming and optical projection. It was clear from simulations without obscuration that the problem was strongly correlated with $\boldsymbol{s}_{v t x}$. The addition of pseudo-points, fabricated points which lay approximately on the space trajectory and were positioned between the vertex and first measurement, significantly improved the track fit by forcing a more careful numerical integration of the equations of motion where the magnetic field was changing rapidly. The improvement in the predictive overlays for measured data was dramatic. 


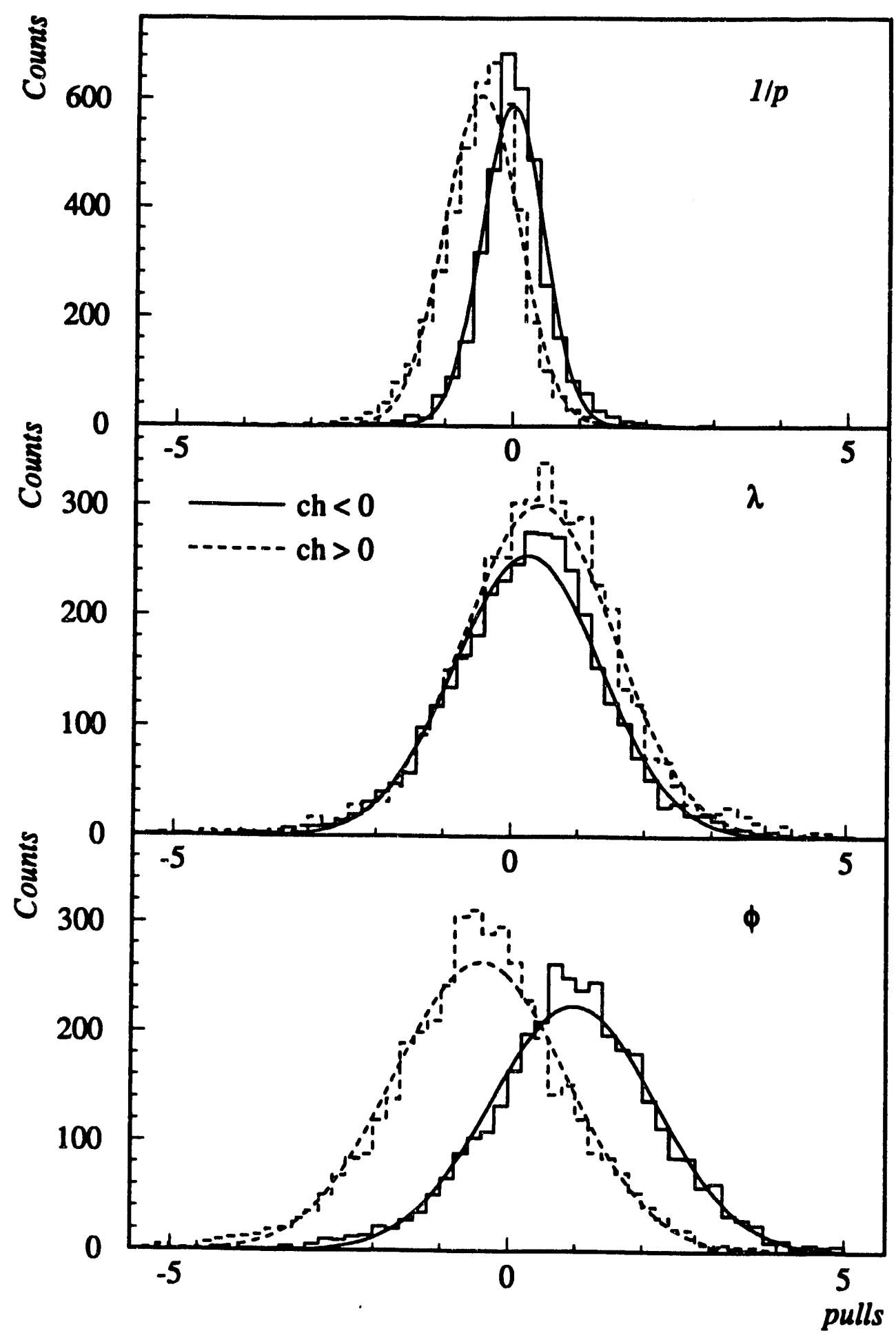

Figure 4.31: Pull quantities for the momentum fit parameters $1 / p, \lambda, \phi$. The solid histograms and Gaussian fits are for negatively charged particles, the dashed histograms and fits for positively charged particles. 
where $L$ is the arc length in the bend plane. If the dip $\lambda$ is small so that $\cos \lambda \simeq 1$ then $L$ is approximately the measured arc length in space. $B$ is the magnetic field strength. If multiple scattering can be neglected, the plot of $\delta p / p$ versus $p$ should be a straight line. This is shown in Figure 4.32. The upper panel is a scatter plot

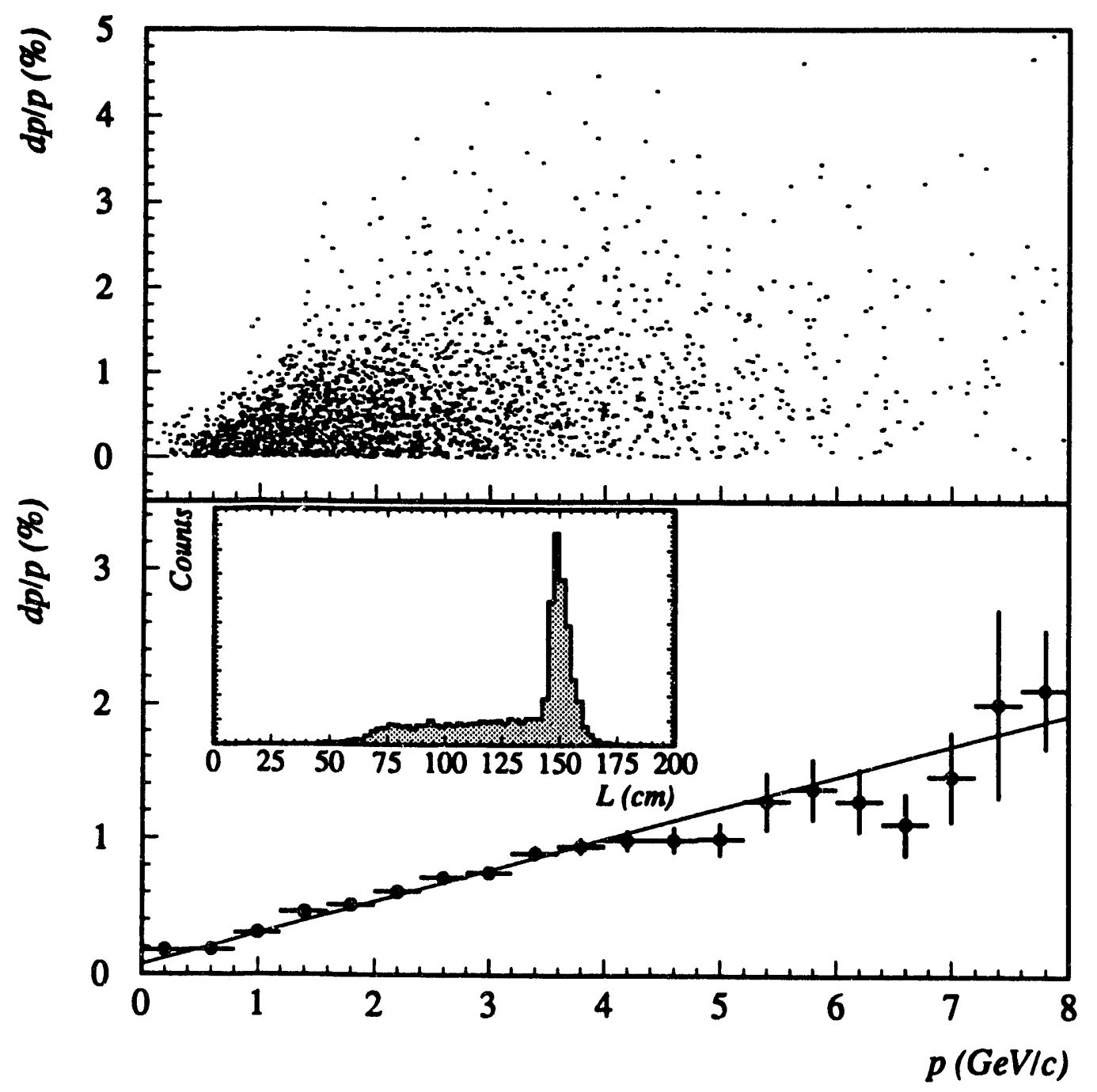

Figure 4.32: Momentum resolution $\delta p / p$ as a function of $p$. The upper panel is a scatter plot, the lower panel a profile histogram which plots the mean and its error in $400 \mathrm{MeV} / \mathrm{c}$ wide momentum bins. The inset histogram shows the arc length distribution. Only tracks in the peak with nearly the same $L$ are included in the plots. The line is the fit from Equation 4.40.

of $\delta p / p$ versus $p$. The inset is the distribution of track lengths $L$ in space from the vertex to the last point. Only particles with $L$ within a narrow window about the sharp peak and with $\cos \lambda \simeq 1$ have been included in the plot. $\delta p$ is determined directly from the input to the simulation and the output from the momentum reconstruction, $\delta p=p_{\text {fit }}-p_{\text {sim }}$. The momenta of tracks in the peak span the 
full momentum range from $p=0$ to $p \simeq 12 \mathrm{GeV} / c$. The lower panel is a profile histogram which plots the mean value and error for $\delta p / p$ in bins which contain particles of nearly the same momentum. The straight line fit yields

$$
\frac{\delta p}{p}=.00075+.0024 p
$$

This value is consistent with a calibration result obtained by shooting beams of known momentum, zero dip and very nearly the same arc length through the streamer chamber [GSI85].

More important for the analysis of charged particle spectra are the resolutions in transverse momentum $p_{T}$ and rapidity $y$. The $p_{T}$ resolution for three-view reconstructions is shown in Figure 4.33. $\delta p_{T}$ is also defined with respect to input

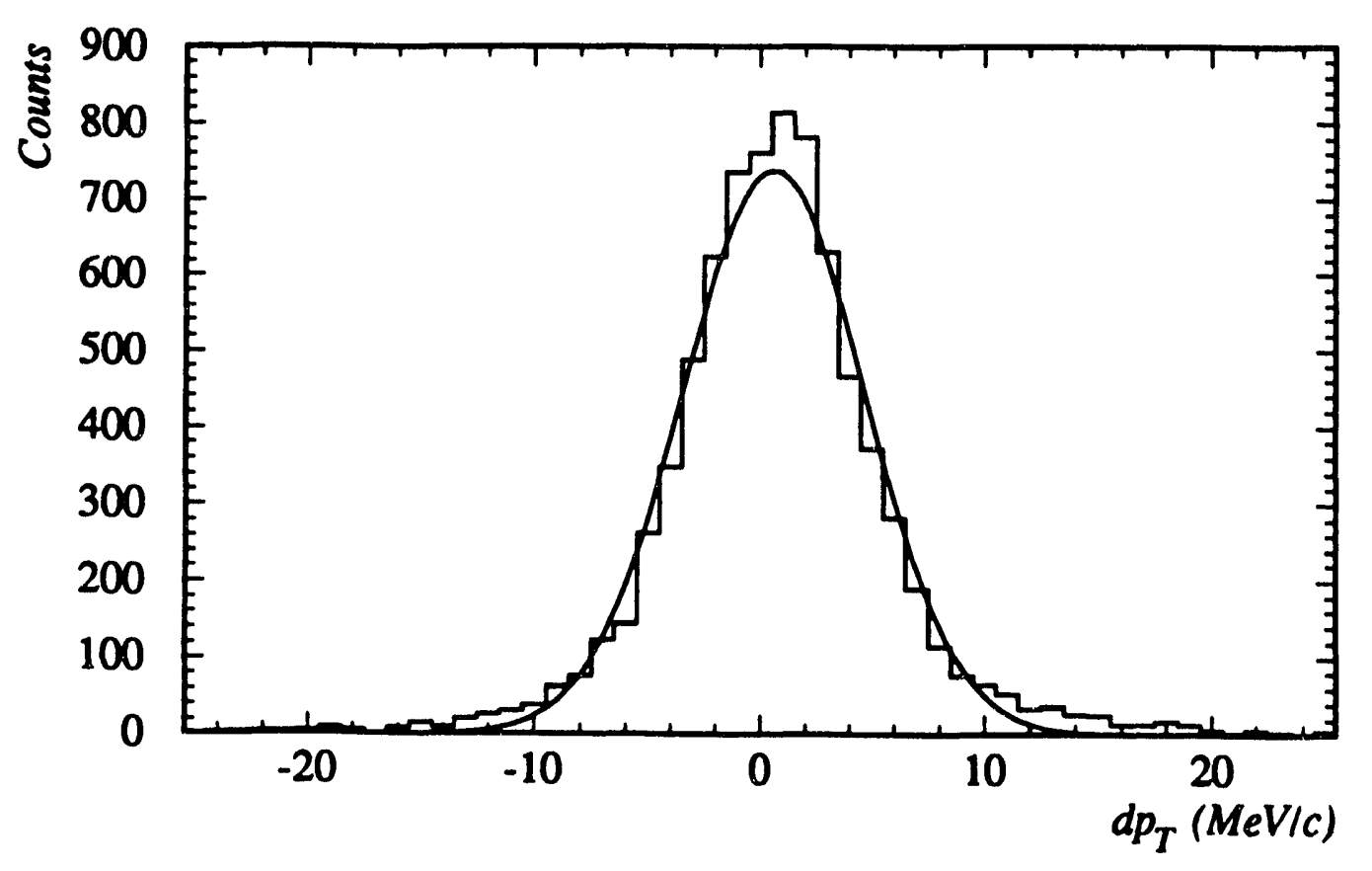

Figure 4.33: Transverse momentum resolution $\delta p_{T}$. The width of the Gaussian fit is $\sigma_{p_{T}}=4.1 \mathrm{MeV} / c$.

to the simulation $\delta p_{T}=p_{T_{f i t}}-p_{T_{\text {sim }}}$. The peak of the distribution is within $1 \mathrm{MeV} / c$ of zero. Its width is $\sigma_{p_{T}}=4.1 \mathrm{MeV} / c$. The resolution for two-view reconstructions is somewhat worse, $\sigma_{p_{T}}=5.3 \mathrm{MeV} / c$. The rapidity difference distribution is also sharply peaked at zero with $\sigma_{y}=.009$ units of rapidity. Again, the resolution is reduced for two-view reconstructions, $\sigma_{y}=.015$ units of rapidity. 


\section{Chapter 5}

\section{Corrections to the Data}

\subsection{Introduction}

In this chapter we discuss three important corrections to the data. First, the geometrical acceptance will be analyzed and a procedure outlined for the track-bytrack calculation of a geometrical weight assigned to observed particles in spectra. Second, a correction for electrons, resulting mainly from $\gamma$ conversion in the target, will be discussed. Third, the influence of non-vertex particles will be examined. A surprisingly large fraction of the charged particles observed in the final state, $\sim 20 \%$, can be attributed to electromagnetic and weak decays, or to secondary hadronic interactions either in the target or detector material.

One feature observed in $p A$ and light projectile-heavy target $A A$ collisions is an enhancement in the transverse momentum distribution of negatively charged hadrons ${ }^{1}$ both for $p_{T}<0.3 \mathrm{GeV} / c$ and for $p_{T}>1.0 \mathrm{GeV} / c$ [Jac9la]. Since electrons are produced predominantly at low $p_{T}$ addressing this question motivates the electron correction. However, this feature is also observed in $\pi^{0}$ transverse momentum spectra [Alb90], where electron contamination is absent, and in negative hadron spectra at midrapidity [Str88], where electrons are relatively scarce. The effect of non-vertex particles, either from electromagnetic or weak decays of primary hadrons, or from secondary interactions induced in the target and detector material, must also be taken into account. Because of the kinematics of two- and three-body decays to charged pions, for example $K_{S}^{0} \rightarrow \pi^{+} \pi^{-}, \eta \rightarrow \pi^{+} \pi^{-} \pi^{0}$, one expects systematically lower transverse momenta for decay pions observed in the final state. The analogous decays to neutral pions $K_{S}^{0} \rightarrow \pi^{0} \pi^{0}, \eta \rightarrow \pi^{0} \pi^{0} \pi^{0}$ occur with significant probability. Surprisingly large primary $\eta / \pi^{0}$ ratios have been reported in $p p$ and $p \bar{p}$ collisions [Jan77], [Aln87], although at much higher energy. Unlike the neutral strange particle decays of the $\Lambda$ and $K_{S}^{0}$, which can be detected even in high multiplicity events [Bar90], the electromagnetic decays of the $\eta$ are impossible to identify so the daughter particles must be included in primary vertex spectra. The leading particle in a hadronic secondary interaction may acquire an

\footnotetext{
${ }^{1}$ Negatively charged particles have been singled out because the experiments which could measure low $p_{T}$ lacked particle identification capability.
} 
additional $p_{T}$ kick. Because of the rapid falloff of the invariant cross section with transverse momentum, a small effect of this kind could have a significant impact at high $p_{T}$. Hence the importance of correcting for non-vertex charged particles in the pion spectra.

A direct measurement of the rapidity distribution of leading protons, either near beam or target rapidity, would complement estimates of the nuclear stopping power inferred from transverse energy distributions for produced particles near midrapidity [PQM88]. We identify the protons with the charge-excess distributions, for example,

$$
d N(A A \rightarrow p) / d y=d N\left(A A \rightarrow h^{+}\right) / d y-d N\left(A A \rightarrow h^{-}\right) / d y .
$$

Because the initial state in ${ }^{32} \mathrm{~S}+{ }^{32} \mathrm{~S}$ collisions is isoscalar, both the yield and phase space dependence of $\pi^{+}$and $\pi^{-}$, the dominant produced charged particles in inelastic $N N$ collisions, should be the same. However distributions for primordial protons obtained by this method will be influenced by the kinematics of the production process and subsequent decay of strange particles. A related effect which influences the proton $d N / d y$ distribution is the asymmetry between $K^{+}$and $K^{-}$ production. $^{2}$ Finally, spectra determined from the charge-excess are sensitive to the intrinsic charge-excess from target fragmentation in secondary hadron-nucleus interactions. This is the second motivation for a careful study of the role played by non-vertex tracks. Final $d N / d y$ distributions will be obtained by extrapolating the corrected spectra down to $p_{T}=0$.

\subsection{Geometrical Acceptance and Geometrical Weight}

The geometrical acceptance is calculated from the good fiducial volume in measurement space. Charged particles were accepted into the analysis if for reconstructed momentum $\vec{p}$ the imageable arc length of the ideally measured equivalent track splmax, defined in Section 4.4.2, was greater than $20 \mathrm{~cm}$ in all three views, and if the associated geometrical weight was not too large, which we quantify below. Recall that splmax has the important virtue of measurement independence, that is, for a correctly identified and carefully measured track it will not depend on the obscuration or the measured arc length. The good fiducial volume finally selected, specified by the analysis cone and the splmax requirement, achieves a reasonable compromise between matching and reconstruction efficiency and phase space acceptance. Note that the forward cone is regarded as an essentially dead region. Naive attempts to improve the efficiency by restricting the fiducial volume, either by enlarging the cone or making a more stringent arc length cut, induced increasingly severe $p_{T}$ cutoffs at the edges of the acceptance, as will become clear below.

\footnotetext{
${ }^{2}$ The $K^{+}$produced in association with $\Lambda$ in processes like $p p \rightarrow p \Lambda K^{+}$is not compensated for because of the absence of analogous channels for $K^{-}$production.
} 
Given the definition of the fiducial volume and splmax, the acceptance calculation is straightforward. The fundamental assumption is that of symmetry about the beam direction. Distributions depending on any equivalent set of phase space variables- $\left(y, p_{T}, m\right),(p, \theta, m),\left(p_{L}, p_{T}, m\right)$-will exhibit no dependence on $\psi$, the azimuthal angle in the transverse momentum plane (see Figure 4.3). For mass hypothesis $m$, charge $q$, and phase space coordinates $\left(y, p_{T}\right)$ the full azimuth is dividec into $n_{\psi}$ equal intervals $\delta \psi=2 \pi / n_{\psi}$. For computational efficiency, a discrete mesh is employed rather than a Monte Carlo procedure. Charged particles are propagated through the streamer chamber magnetic field and optically projected from chamber space to the measurement spaces of cameras 1, 2 and 3. Neglecting pu-turbations like energy loss and multiple scattering, the imageable projected arc length splmax is a single-valued function $f\left(y, p_{T}, m, \psi\right)$. If splmax $>20 \mathrm{~cm}$ in all three views, it is assumed that the charged particle will be detected and measured, in at least the two views required for momentum reconstruction. This assumption makes a statement about efficiency as well as providing a criterion for acceptance. It will be explicitly checked below. For a given value of $\left(y, p_{T}\right)$, at the midpoint of each interval $\delta \psi$, the acceptance criterion is evaluated and, if satisfied, the number of accepted particles $n_{a c c}\left(y, p_{T}\right)$ is incremented. This procedure leads to the definition of a geometrical weight $w\left(y, p_{T}\right)$ :

$$
w\left(y, p_{T}\right)=n_{\psi} / n_{a c c}\left(y, p_{T}\right) \text {. }
$$

The geometrical acceptance is defined as all $\left(y, p_{T}\right)$ such that $w\left(y, p_{T}\right)<8.0$. The upper bound on $w$ was selected to avoid regions of phase space where the weights change too rapidly and also to suppress large statistical fluctuations which can result from the inclusion of a few counts with excessively heavy weights.

The geometrical accentance for $\pi^{-}$is shown in Figure 5.1. The upper figure is a lego plot of the geometrical weight, calculated at bin centers, as a function of $y$ and $p_{T}$. In the lower figure, which shows the edges of the acceptance more clearly, the size of the boxes is proportional to the weight. Note the broad valley in the lego plot where $w\left(y, p_{T}\right)<3.0$, corresponding to the region of high efficiency. The sweeping ridge at the edge of the valley approximately traces a line of constant $\theta_{l a b}$ in $\left(y, p_{T}\right)$ space. In the rectangular phase space region $0.6<y<2.8,0.05<p_{T}<2.0 \mathrm{GeV} / c$, the maximum weight is $w\left(y, p_{T}\right) \simeq 6$.

The sharp high-p $p_{T}$ cutoff with increasing $y$, evident in the lower figure, occurs because for $p_{T}>m_{\pi}, y \simeq$ pseudorapidity $\eta=-\ln (\tan \theta / 2)$. For a given rapidity $y$, the emission angle in the laboratory is independent of $p_{T}$. Forward of midrapidity, particles are concentrated at very srnall angles. For $y>y_{\text {mid }}=3.0, \theta_{l a b}<5^{\circ}$. However, increasing $p_{T}$ with $y$ held constant increases the rigidity, making it difficult for the magnetic field to sweep charged particles out of the cone so they can be detected. The acceptance edge at low rapidity for $p_{T}>0.2 \mathrm{GeV} / c$ corresponds to $\theta_{\text {lab }}>60^{\circ}$. For $p_{T}<0.2$, there is a low momentum cutoff at $p \simeq 70 \mathrm{MeV} / c$. The $\pi^{+}$acceptance is nearly the same, except it does not extend quite as far in $y$ because of imperfect symmetry in the construction of the analysis cone.

Identical plots for protons are shown in Figure 5.2. The lego plot exhibits a similar broad valley of good efficiency. A sharper ridge structure indicates that 

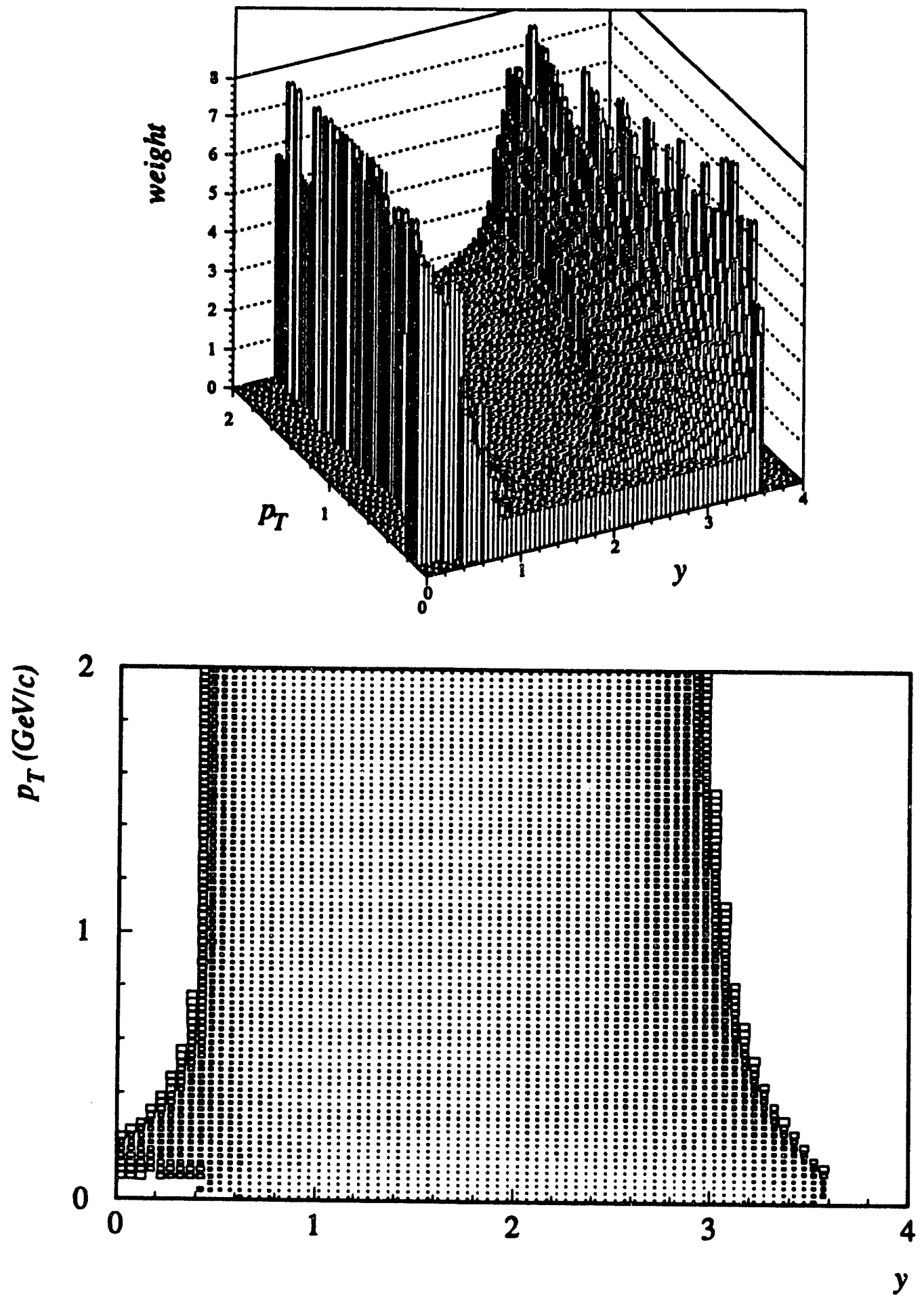

Figure 5.1: Geometrical $\left(y, p_{T}\right)$ acceptance for $\pi^{-}$. The upper figure plots the weights at the bin centers. In the the lower figure, which displays the edges more clearly, the box size is proportional to the weight. The $\pi^{+}$acceptance is equivalent except for small differences in the locations of the edges defined by $w\left(y, p_{T}\right)=8.0$. 

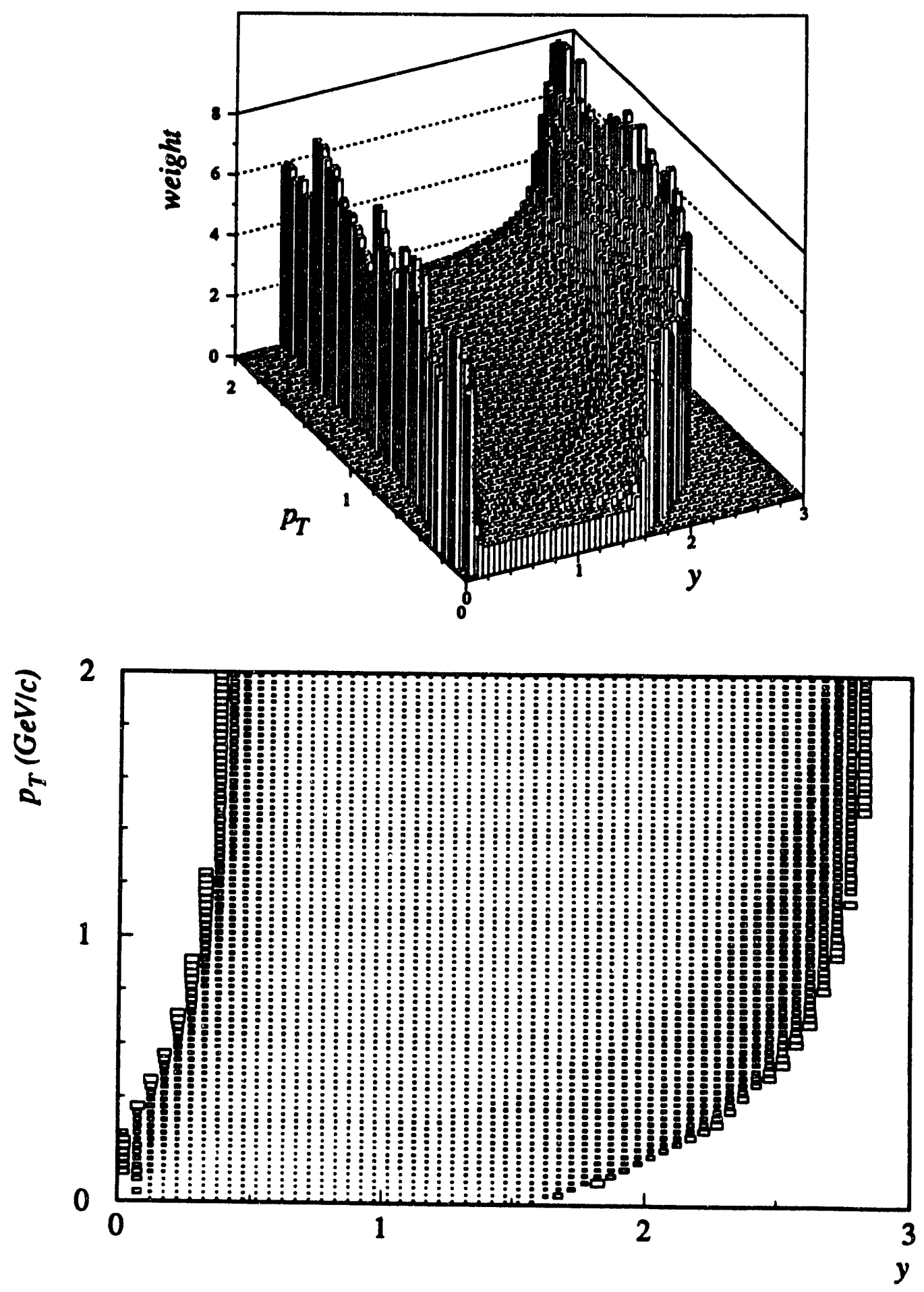

Figure 5.2: Geometrical $\left(y, p_{T}\right)$ acceptance for protons. The rapidity-dependent low- $p_{T}$ cutoff is a consequence of the large proton mass relative to the $p_{T}$ scale. 
the proton acceptance is more clearly the consequence of an angle cut. The proton acceptance edge at low $y$ and low $p_{T}$ has the same structure and similar kinematic origins to the edge in the $\pi^{-}$acceptance. Because half of the phase space satisfies $p_{T}<m_{p}$ there is a pronounced difference at low $p_{T}$, increasing with rapidity. For $y>1.6$, decreasing $p_{T}$ with $y$ held constant leads to a rapid reduction in $\theta_{\text {lab }}$ to very small angles. The diminution in rigidity cannot compensate for this rapid change in the angle of emission. The influence of the strongly rapiditydependent low-p $p_{T}$ cutoff may be somewhat diminished since the mean transverse momentum $<p_{T}>$ for hadrons is known to increase significantly with mass in both pp [Eis79], [Ale90] and $A A$ [Abb90] collisions over a wide range of energies. A recent measurement by the NA35 collaboration [Wen90] has reported a $<p_{T}>=660$ $\mathrm{MeV} / \mathrm{c}$ for protons indirectly identified from the charge-excess distribution in the same experiment, although for a different trigger and a different data reduction technique.

In lower energy $A A$ collisions, where high multiplicity events are still dominated by incident nucleons rather than produced particles and where the kinematic focusing into the forward cone is not so dramatic, the selection of tracks within the $\left(y, p_{T}\right)$ acceptance which participate in the physics analysis and the assignment of their respective weights is also based on the assumption of rotational symmetry about the beam. The canonical approach to correct for detection efficiency is to identify an interval in $\psi$ of unbiased, near $100 \%$ efficiency with a plateau in the distribution $d N / d \psi$ as a function of $\psi$ [Str84]. A characteristic feature of these distributions is the existence of camera holes for values of $\psi$ which correspond to trajectories pointing towards/away from the cameras. The role of obscuration is evident from the typically greater broadness and depth of the away from camera hole. Once a plateau in the $d N / d \psi$ distribution has been defined with width $\delta \psi$, tracks within the plateau are included in the analysis and assigned weights

$$
w_{\psi}=\frac{2 \pi}{\delta \psi}
$$

These are analogous to the geometrical weights $w\left(y, p_{T}\right)$ calculated above. This data selection procedure has the deleterious effect of sacrificing a significant fraction of the measured data sample.

The canonical approach assumes that the positions of the $\psi$ plateaus and, correspondingly, the values of the weights $w_{\psi}$ are slowly changing functions of phase space. This approach has been adopted for the NA35 film data [Wen90] and implemented by dividing phase space and defining track selection plateaus as a function of $\left(p, \theta_{l a b}\right)$.

For CCD-imaged data the plateaus in the $d N / d \psi$ distribution have a very strong phase space dependence and can lead to camera holes which are exceedingly broad, even with perfect detection efficiency outside of the forward cone. This is illustrated in Figure 5.3. The first three figures in each column are scatter plots of splmax as a function of $\psi$, in camera views 1,2 , and 3 respectively, for momentum $p=1.5 \mathrm{GeV} / c$. Each column is plotted for a different polar angle, $\theta_{\text {lab }}=2^{\circ}$, $10^{\circ}$, and $20^{\circ}$, respectively. These values of $\left(p, \theta_{l a b}\right)$ correspond to $\left(y, p_{T}\right)$ values of 


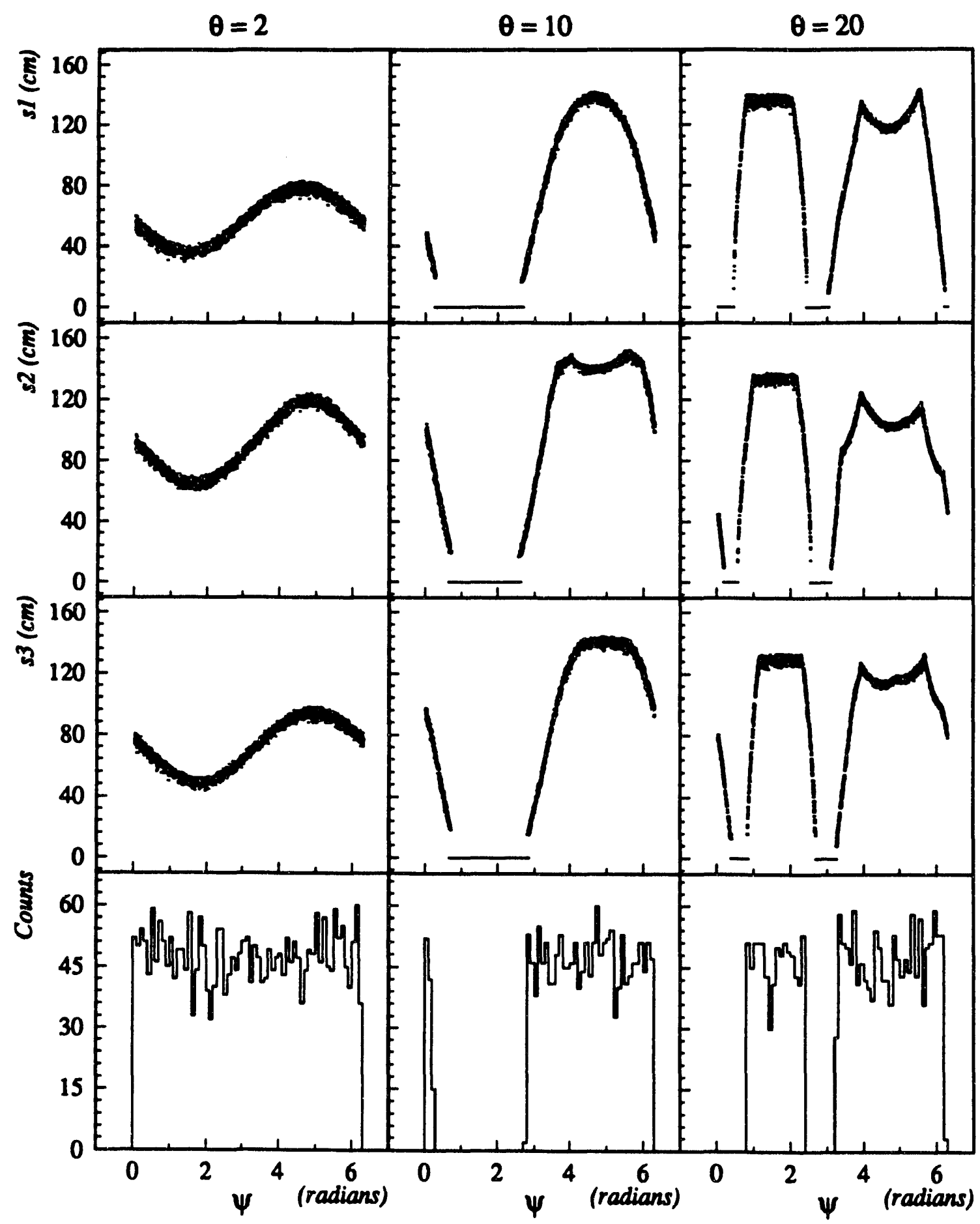

Figure 5.3: Projected arc length distributions splmax as a function of $\psi$ for $p=1.5$ $\mathrm{GeV} / c$ and $\theta_{\text {lab }}=2^{\circ}, 10^{\circ}$, and $20^{\circ}$. The values of splmax obtained for camera views 1,2 , and 3 are denoted $s_{1}, s_{2}$, and $s_{3}$, respectively. Also shown are the subsets of $\psi$ which satisfy $s_{1,2,3}>20 \mathrm{~cm}$. The locations and extents of the camera holes change dramatically with $\theta_{l a b}$. 
$\simeq(3.0,50 \mathrm{MeV} / c),(2.3,250 \mathrm{MeV} / c),(1.7,500 \mathrm{MeV} / c)$. The last figure in each column shows the subset of $\psi$ which satisfies the acceptance criterion. Recall that we measure $\psi$ from the positive $z$ axis. $\psi=0(\pi)$ for particles with transverse momentum pointing toward(away from) the $z$ axis, approximately toward(away from) the cameras. For each value of $(p, \theta)$ a Monte Carlo procedure is employed to sample randomly in $\psi$ such that $d N / d \psi$ is flat. The calculation shown assumes a charge $q=-e$. The smearing in the scatter plots occurs because the calculation of splmax employed for these plots is not exact-the arc length is computed from the first point in measurement space excluding points which image into the central cone, rather than from the track-fiducial volume intersection point. The arc length drops abruptly to zero when too few points remain outside of the cone to perform a circle fit.

The structure in the scatter plots is predominantly a rightside/wrongside effect. The negative particle rightside covers the domain $\pi<\psi<2 \pi$. See page 66 and Figure 4.4. Note the approximate symmetry about the chamber midplane, located at $\psi=\pi / 2, \psi=3 \pi / 2$, a separate symmetry for rightside and wrongside particles. For $\theta_{\text {lab }}=2^{\circ}$, there is full acceptance for all values of $\psi$. The oscillation in splmax has a relatively small amplitude. The fluctuations in the counts vs $\psi$ distribution are statistical. Note that for $\theta_{l a b}=0^{\circ}$, the transverse momentum is identically zero and the $\psi$ dependence disappears entirely from the problem. As $\theta_{\text {lab }}$ increases to $10^{\circ}$, not enough to initially point wrongside particles out of the cone, a huge camera hole develops on the wrongside. As $\theta_{\text {lab }}$ increases further, the wrongside acceptance recovers because the initial direction of the charged particle permits unobscured imaging for most wrongside values of $\psi$. Camera holes can be observed for both toward and away from values of $\psi$. The spread of imageable arc lengths is large with splmax ranging from 0 to $\simeq 150 \mathrm{~cm}$. Note that there can be zero, one, or two camera holes depending on $\left(p, \theta_{l a b}\right)$.

Because of the sensitivity of both the location and the width of the $d N / d \psi$ plateau on phase space, a weighting procedure based on relatively coarse binning would be inappropriate. Instead we calculate a track-by-track geometrical weight. This method has two advantages. First, it eliminates artificial or incorrect weight assignments in spectra due to rapid variations in the detection efficiency. Second, it minimizes the amount of data which must be excluded from the physics analysis. From the bottom three plots in Figure 5.3 it is clear that application of the canonical plateau method would reject $\sim 1 / 2$ the measured data.

The geometrical weight calculation is identical to the acceptance calculation except that the reconstructed momenta, rather than any bin center or any randomly sampled momentum values within a phase space bin, are used as initial conditions for the splmax computation. The $\psi$ segmentation is made finer to reduce the statistical error. For reconstructed $(p, \lambda, \phi)$ charged particles are propagated through the magnetic field, projected to the measurement space and subjected to the fiducial volume cut splmax $>20 \mathrm{~cm}$ in all three views. Note that the cut is independent of whether a track has been measured in two or three views. Approximately $90 \%$ of all reconstructed tracks are accepted. Tracks which satisfy the three-view acceptance criterion are symmetrized with respect to $\psi$ for reconstructed $(p, \theta)$. For the 
weight calculation the $2 \pi$ transverse azimuth was divided into $n_{\psi}=500$ intervals of $\delta \psi=0.76^{\circ}$. This guaranteed a statistical error of less than $\simeq 2 \%$ for the largest weights. Just as for the case of the acceptance, $s p l m a x=f(p, \theta, \psi, q)$ is calculated and $n_{\text {acc }}$ incremented if the fiducial cut is satisfied. This leads to the definition of the geometrical weight $w_{i}$ of track $i$ :

$$
w_{i}(p, \theta, q)=n_{\psi} / n_{a c c}(p, \theta, q) .
$$

The geometrical weight depends only on the reconstructed momentum and charge. Its error can be calculated by estimating the uncertainty in $n_{\text {acc }}$. We assume that the acceptance decision splmax $>20 \mathrm{~cm}$ may have been incorrectly made once at the edge of each potential camera hole. Averaging the deviations of the high and low weights obtained from $n_{a c c} \rightarrow n_{a c c} \pm 2$ gives the error.

The error estimate was checked by comparing the result, for a number of values of $(p, \theta)$, to the weight obtained by an analogous calculation which used a Monte Carlo procedure to sample randomly in $\psi$ for given $(p, \theta)$. This calculation used a much larger number of trials $N$ and was assumed to converge to the true weight like $1 / \sqrt{N}$. The geometrical weights calculated by both methods agreed within the error estimate, but the discrete calculation converged to the correct value much faster. Examination of the measurement space overlays, which are calculated from the reconstructed momenta in exactly the same manner as splmax, showed no systematic deviations of the predicted measurement space trajectories, and by implication splmax, from the measured data. There is no evidence for any systematic error in the computation of the track-by-track geometrical weights.

In order to test the fundamental efficiency assumption-that tracks which satisfy splmax $>20 \mathrm{~cm}$ in three views will be measured in at least two and successfully matched and reconstructed without suffering losses which introduce a bias-we applied the complete data reduction procedure to simulated data. The "measured" spectra employed in the matching efficiency analysis of Section 4.8 , which have folded in the effects of scanning, matching, and reconstruction inefficiencies, were inverted with the aid of the computed geometrical weights. Figure 5.4 compares the normalized rapidity distribution of negative hadrons $d N / d y$ of the inverted, "measured" distribution to a reference spectrum, obtained directly from the input FRITIOF distribution. The reference spectrum is completely unfiltered. Both spectra contain the same 200 events, a sample of the same statistical significance as our real measured data. The contents and error bar of histogram bin $h_{i}$ are

$$
h_{i}=\sum_{j=1}^{N_{i}} A w_{j}, \quad \delta h_{i}=\sqrt{\sum_{j=1}^{N_{i}}\left(A w_{j}\right)^{2}},
$$

respectively. $N_{i}$ is the number of tracks which land in bin $i$ and $A$ normalizes the distribution so that it is a rapidity density with integrated value equal to the average negative multiplicity.

At this statistical level the reference spectrum is subject to large statistical fluctuations. The inverted spectrum, which because of the rejection in the simulated 


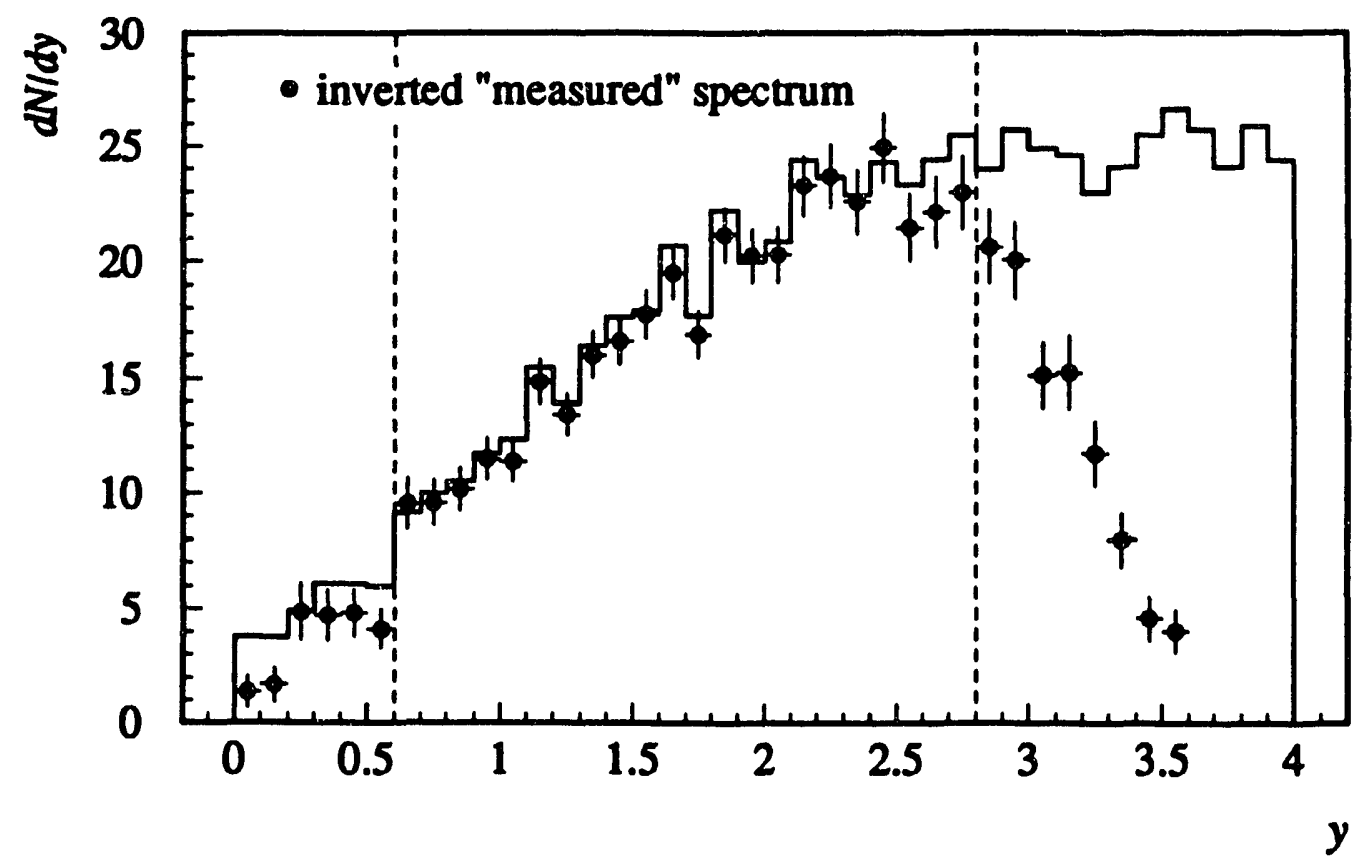

Figure 5.4: Comparison of inverted, "measured" distribution to the FRITIOF reference spectrum. The reference spectrum is obtained directly from the input distribution. The dashed lines indicate the efficiency-restricted acceptance.

data of tracks from the forward cone contains only 45\% of the counts, not only is consistent with the input distribution but also preserves the fluctuations. For $y>3.0$ the effect of the rapidity-dependent, high-p $p_{T}$ acceptance cutoff is evident. The two rapidity bins for $2.8<y<3.0$ deviate systematically indicating that the efficiency hypothesis has not been satisfied at the high rapidity edge of the geometrical acceptance. This might also be the result of an inconsistency of the track match momentum cut at $p=16 \mathrm{GeV} / c$ which cuts into the acceptance at $y=2.8$ for $p_{T}>1.5 \mathrm{GeV} / c$, although the yield at high $p_{T}$ is probably too low to account for it alone. A similar effect may be occurring at the low edge of the geometrical acceptance at $y \simeq 0.5$. For this reason we will restrict the physics analysis to $0.6<y<2.8$ with rapidity calculated assuming the pion mass. ${ }^{3}$ The overall normalization appears to be correct which, combined with the $\simeq 90 \%$ matching and reconstruction efficiency estimate from Section 4.8, suggests that most of the losses occur at the edges of the geometrical acceptance. The close agreement between the inverted and reference distributions also implies that the compromised and spurious tracks do not distort spectra.

The final acceptance for pions and protons, taking into account the limitations imposed by efficiency considerations, is shown in Figure 5.5. The $\pi$ acceptance, indicated by the thick dashed line, is rectangular in $\left(y, p_{T}\right)$ space. The low- $p_{T}$ cut

\footnotetext{
${ }^{3}$ We will strictly adhere to this acceptance for $x^{-}$transverse momentum spectra and also for the restrictions it imposes on the proton acceptance. For the $\pi^{-} d N / d y$ distribution we will relax the lower bound to $y=0.4$. This is still consistent with the comparison in Figure 5.4 and the geometrical acceptance shown in Figure 5.1.
} 


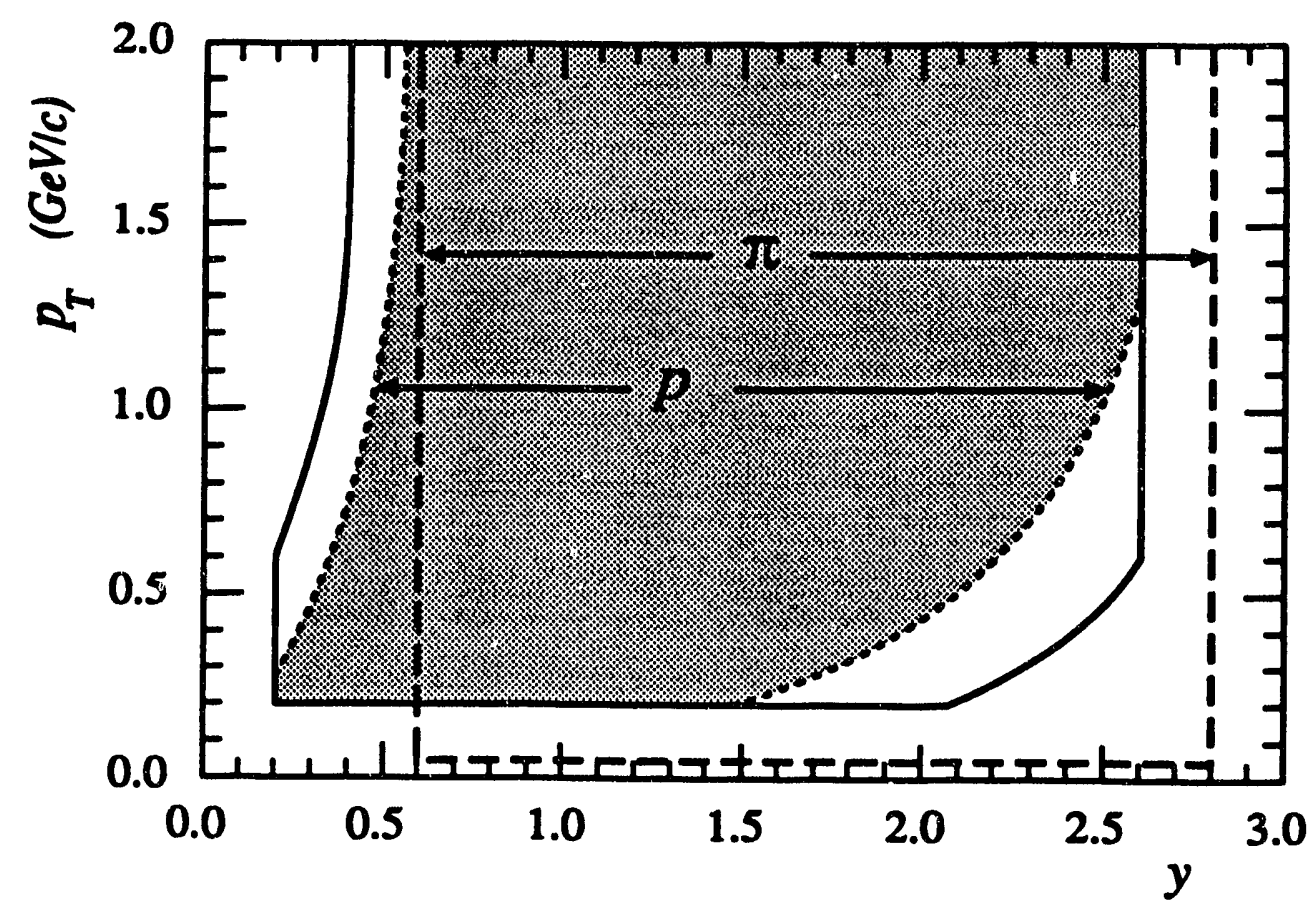

Figure 5.5: Final pion and proton acceptance in $\left(y, p_{T}\right)$ space. The solid line is the proton geometrical acceptance. The shaded region is the restricted proton acceptance implied by the rectangular pion acceptance.

at $50 \mathrm{MeV} / c$ anticipates a result from Section 5.3-the background from conversion $e^{-}$exceeds the hadronic signal for $p_{T}<50 \mathrm{MeV} / c$. The proton acceptance, represented by the solid line, has been truncated at $y=0.2$ and $y=2.6$ in rapidity. If the $<p_{T}>\sim 600 \mathrm{MeV} / c$, the low(high)-p $p_{T}$ acceptance cutoff at $y=0.2(2.6)$ still permits approximately half the transverse momentum to be measured for the purpose of extrapolation. The low-p $p_{T}$ cut at $200 \mathrm{MeV} / c$ obviates the need for an $e^{+}, e^{-}$correction to the proton spectra and suppresses negative fluctuations in the charge-excess distributions which result from kinematic asymmetries in the weak decay $\Lambda \rightarrow p \pi^{-}$. This point will be clarified in Section 5.4. Because of the high proton $\left\langle p_{T}\right\rangle$, this acceptance cutoff still takes a relatively small bite out of the available phase space. A low- $p_{T}$ cut at $200 \mathrm{MeV} / c$ would bound a rectangular phase space region for $0.4<y<2.0$. Since the average rapidity shift imparted to inelastically interacting leading protons in $p p$ collisions at comparable energies is $\simeq 1-1.5$ units of rapidity [Bus84], we can measure the proton transverse momentum spectrum over the interval of rapidity where the yield is expected to be greatest.

However, since we extract proton observables from the pion-dominated chargeexcess distributions, the proton acceptance must be reduced still further. The dotted lines in Figure 5.5 map lines of constant pion rapidity $y_{\pi}=c$ to correspon ling values of the proton rapidity $y_{p}$ calculated for different values of $p_{T}$;

$$
y_{p}=f\left(m_{p}, p_{T}, p_{L}\left(m_{\pi}, p_{T}, y_{\pi}=c\right)\right)=f\left(m_{p}, p_{T}, m_{\pi}, y_{\pi}=c\right) .
$$


The shaded region is bounded by the lines $y_{p}=f\left(p_{T}, y_{\pi}=0.6\right)$ and $y_{p}=$ $f\left(p_{T}, y_{\pi}=2.8\right)$ suggested by the efficiency-restricted $\pi$ acceptance. Note the strong $p_{T}$ dependence of the rapidity shifts for the different mass assumptions. This is another consequence of the large proton mass relative to the $p_{T}$ scale. Even neglecting this efficiency effect, a cul would have be made on rapidity calculated with the pion mass to prevent a false signal in the charge-excess distributions arising from a small difference in acceptance for $\pi^{+}$and $\pi^{-}$. A cut requiring $y\left(m_{\pi}, p_{T}, p_{L}\right)<3.0$ would ensure equivalence of the $\pi^{-}$and $\pi^{+}$acceptance. The most realistic assumption, that the detection efficiency for pions and protons as a function of $(p, \theta)$ is the same, argues for the restricted proton acceptance in $\left(y, p_{T}\right)$ space. This limits the rapidity range for an unbiased proton $p_{T}$ spectrum to $0.6<y_{p}<1.4$ and necessitates a rapidity-dependent extrapolation to obtain the $d N / d y$ distribution for participant protons.

\subsection{Electron Correction}

As is well known, photons are produced copiously in hadron-hadron collisions by the electromagnetic decay of directly produced hadrons. The inclusive yield is comparable to that observed for pions [Fer84]. The decay processes primarily responsible for the large yield are:

$$
\begin{aligned}
\pi^{0} & \rightarrow \gamma \gamma \\
\eta & \rightarrow \gamma \gamma \\
\eta & \rightarrow 3 \pi^{0} \\
\eta & \rightarrow \pi^{+} \pi^{-} \pi^{0}
\end{aligned}
$$

with the subsequent $\pi^{0}$ decays. As was mentioned in Section 5.1, the $\eta$ contribution may be important. In nucleus-nucleus collisions with massive, high- $Z$ targets conversion electrons produced by the process $\gamma \rightarrow e^{+}+e^{-}$can have a significant impact on the charged particle spectra.

In the absence of electron/hadron discrimination by time-of-flight or $d E / d x$ the electron contamination can be calculated starting from either the observed $\gamma$ flux [Alp75], a measurement of the charged or neutral pion differential cross sections [Wen90], or from the $\pi^{0}$ and $\eta$ distributions generated by a Monte Carlo simulation which well approximates the data. A $\pi^{0}$ based calculation has the advantage of including a large part of the $\eta$ contribution but the $\pi^{0}$ cross sections in nucleusnucleus collisions have not been well measured below $p_{T}=400 \mathrm{MeV} / c$ [Alb90]. In an isoscalar system like ${ }^{32} \mathrm{~S}+{ }^{32} \mathrm{~S}$ the direct(i.e, parton/string/strong resonance) $\pi^{0}$ production will be the same as the charged pion production. However, a correction based on assuming that the neutral pion $\left(y, p_{T}\right)$ phase space distribution is identical to the measured charged pion distribution must still make assumptions based on models or $p p$ data about the relative yield $\pi^{0} / \pi^{-}$and the effect of the $\eta$ decay kinematics on the $\pi^{0}$ spectrum. We have calculated the correction from FRITIOF including photons generated from the electromagnetic decays of all the 
stable particles. Electrons produced by the Dalitz decay $\pi^{0} \rightarrow e^{+} e^{-} \gamma$ have been included as well. Possible systematic errors introduced by employing the FRITIOF input spectra will be addressed below.

An electron correction table $c_{i j}$ was calculated for bins in $\left(y, p_{T}\right)$ space from the ratio of negative hadron counts $h_{i j}\left(y, p_{T}\right)$ to the sum of hadron plus electron counts $e_{i j}\left(y, p_{T}\right)$,

$$
c_{i j}=\frac{h_{i j}}{h_{i j}+e_{i j}}
$$

with the conversion electron counts appropriately weighted by the conversion probability $P$. The $c_{i j}$ range from zero to one. The bin sizes were 0.1 units in rapidity by $50 \mathrm{MeV} / c$ in transverse momentum. $\pi^{-}, K^{-}$, and $\bar{p}$ were included in the hadron $\left(y, p_{T}\right)$ distribution with all rapidities calculated assuming the charged pion mass. The electron phase space distribution included conversion electrons from $\gamma$ decay plus directly produced electrons from the Dalitz decay modes of the $\eta$ and the $\pi^{0}$. Physics routines to calculate the total cross section, mean free path and probability for photon pair production and to sample the secondary electron/positron energies were extracted from GEANT [Bru87], the CERN detector simulation package. They employ a modified version of the method of Butcher and Messel [But60] to sample from the Coulomb corrected Bethe-Heitler differential cross section $d \sigma(Z, E, \epsilon) / d \epsilon$ for the production of an electron/positron pair, one of which has energy $\epsilon E$, from an incident photon of energy $E$ [For78]. $Z$ is the nuclear charge.

Each $\gamma$ was converted $n_{\gamma}$ times and weighted by $P / n_{\gamma}$ in order to smooth statistical fluctuations. However, the statistical error of the correction where the correction is large depends strongly on the number of hadron counts per bin, which demands a large number of Monte Carlo events. A sufficient number of events was run to populate the low $p_{T}$ bins where the hadrons are relatively rare. Since the numbers of both hadrons and electrons are seldom both small, the statistical error of the correction can be estimated directly by straightforward error propagation [Jam80].

The only important conversion material is the $1.18 \mathrm{gm} / \mathrm{cm}^{2}(.57 \mathrm{~cm})$ thick ${ }^{32} \mathrm{~S}$ target. All other material in the $\gamma$ paths is much thinner and has much lower nuclear charge. The typical conversion probability, averaged over all $\gamma$ incident energies, is $\simeq 2.5 \%$. This implies that the Dalitz decay contributes $\sim 1 / 4$ of the $e^{-}$yield and should not be ignored. The decay and conversion kinematics strongly focus the produced electrons at low transverse momentum. For $\pi^{0}$ parents with $p_{T}<250 \mathrm{MeV} / c$, the daughter electron transverse momenta fall approximately exponentially with a mean value of $\left\langle p_{T}>\simeq 50 \mathrm{MeV} / c\right.$. For $\pi^{0}$ parents with $p_{T}>1 \mathrm{GeV} / c$ the daughter electron transverse momenta are more peaked than exponential at low $p_{T}$. Although spanning the full, allowable kinematic range from $0<p_{T}<p_{T, \pi^{0}}$ the number of electrons descending from high transverse momentum $\pi^{0}$ s which have $p_{T}$ in the heart of the charged pion spectrum is still small. The kinematics argue that the correction should be somewhat insensitive to the $\pi$ momentum spectrum at extreme values where data [Ake90], [Str88] departs from models based on the superposition of $p p$ physics. 
Figure 5.6 compares the calculated $e^{-}$and FRITIOF $\pi^{-}$normalized rapidity distributions $d N / d y$ integrated over all transverse momenta $0.0<p_{T}<2.0 \mathrm{GeV} / c$. The ordinate is plotted logarithmically because of the more than order of magnitude difference in the yields over most of the rapidity domain. Electron rapidity $y_{e}$ is calculated assuming the pion mass:

$$
y_{e}=f\left(m, p_{L}, p_{T}\right) \rightarrow f\left(m_{\pi}, p_{L}, p_{T}\right)
$$

The solid line, symmetric about midrapidity, is for $\pi^{-}$. Four electron distributions are drawn, showing the dependence on low transverse momentum cuts requiring $p_{T}>0,25,50,75 \mathrm{MeV} / c$ respectively. Without a $p_{T}$ cut the electron yield at target rapidity is comparable to the charged pion yield but falls off rapidly by $y=1$. A $p_{T}$ cut requiring $p_{T}>50 \mathrm{MeV} / c$ completely suppresses the electron peak and leads to a fairly flat distribution for $0.2<y<4.0$ at the level of $\sim 1 / 2$ of an electron per unit of rapidity. This cut only affects the normalization of the $\pi^{-}$rapidity distribution, by $\sim 4 \%$, since the ratio of the pion distributions with and without the cut is almost flat for $0.6<y<5.4$.

The influence of the electron contamination on the transverse momentum spectrum is shown in Figure 5.7, which plots the ratio of the electron yield to the negative hadron yield as a function of $p_{T}$, integrated over rapidity for $0.0<y<4.0$. The contamination is $55 \%$ in the first bin, $15 \%$ in the second bin, $6 \%$ in the third bin and falls to less than $1 \%$ for $p_{T}>250 \mathrm{MeV} / c$. The rapidity dependence of the effect was explored by plotting the ratio for different low rapidity cuts at $y>0.5$, 1.0 and 2.0 . These have been omitted from Figure 5.7 because the dependence on the low rapidity bound was weak except for the first two bins. At midrapidity, $2.0<y<4.0$, the contamination is $33 \%, 12 \%$ and $5 \%$ in the first three bins respectively. Both the rapidity distribution and relative transverse momentum yields point out the difficulty of measuring spectra down to very low $p_{T}$ without particle identification. This is the reason we have excluded transverse momenta below $\mathbf{5 0}$ $\mathrm{MeV} / c$ from our physics acceptance.

Representative elements of the correction table are shown as a series of onedimensional histograms in Figure 5.8. Each histogram plots the $c_{i j}\left(y, p_{T}\right)$ defined in Equation 5.6 as a function of rapidity for selected bins of transverse momentum. The labels identify the bin boundaries of the $50 \mathrm{MeV} / c p_{T}$ bins used for the correction. The corrections are still relatively large until $p_{T} \sim 150 \mathrm{MeV} / c$. For $p_{T}>500 \mathrm{MeV} / c$ the probability weighted electron yield is less than $\simeq 1$ count per bin, leading to correction factors very close to unity. If either $h_{i j}$ or $e_{i j}$ was zero, $c_{i j}$ was set to one. The statistical error on the correction factors can be estimated by

$$
\sigma_{c}{ }^{2}=\frac{e^{2}}{(h+e)^{4}} h+\frac{h^{2}}{(h+e)^{4}} \sum_{i}\left(P_{i} / n_{\gamma}\right)^{2}
$$

where the terms $h$ and $\sum\left(P / n_{\gamma}\right)^{2}$ are estimates to $\sigma_{h}{ }^{2}$ and $\sigma_{e}{ }^{2}$ respectively. For $n_{\gamma}=10$ and for 10,000 Monte Carlo events the statistical errors were less than $\simeq 1 \%$ everywhere in the physics acceptance. 


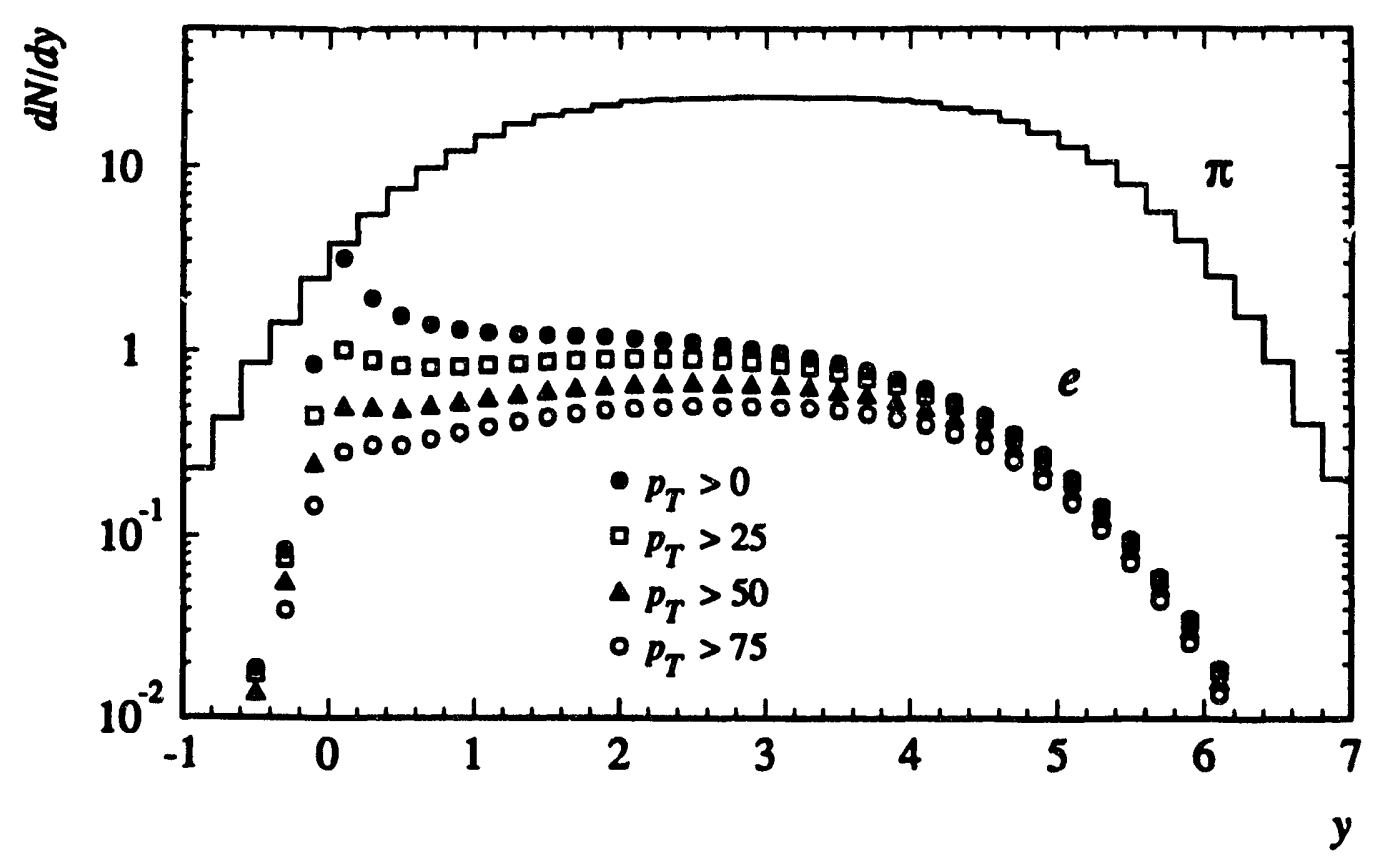

Figure 5.6: Normalized electron and pion rapidity distributions. The solid line, symmetric about midrapidity, is for $\pi^{-}$. The influence of transverse momentum cuts on the electron $d N / d y$ distribution is shown for $p_{T}>0,25,50,75 \mathrm{MeV} / c$. For $p_{T}>50 \mathrm{MeV} / c$ the electron yield is fairly flat from $0.2<y<4.0$ units of rapidity.

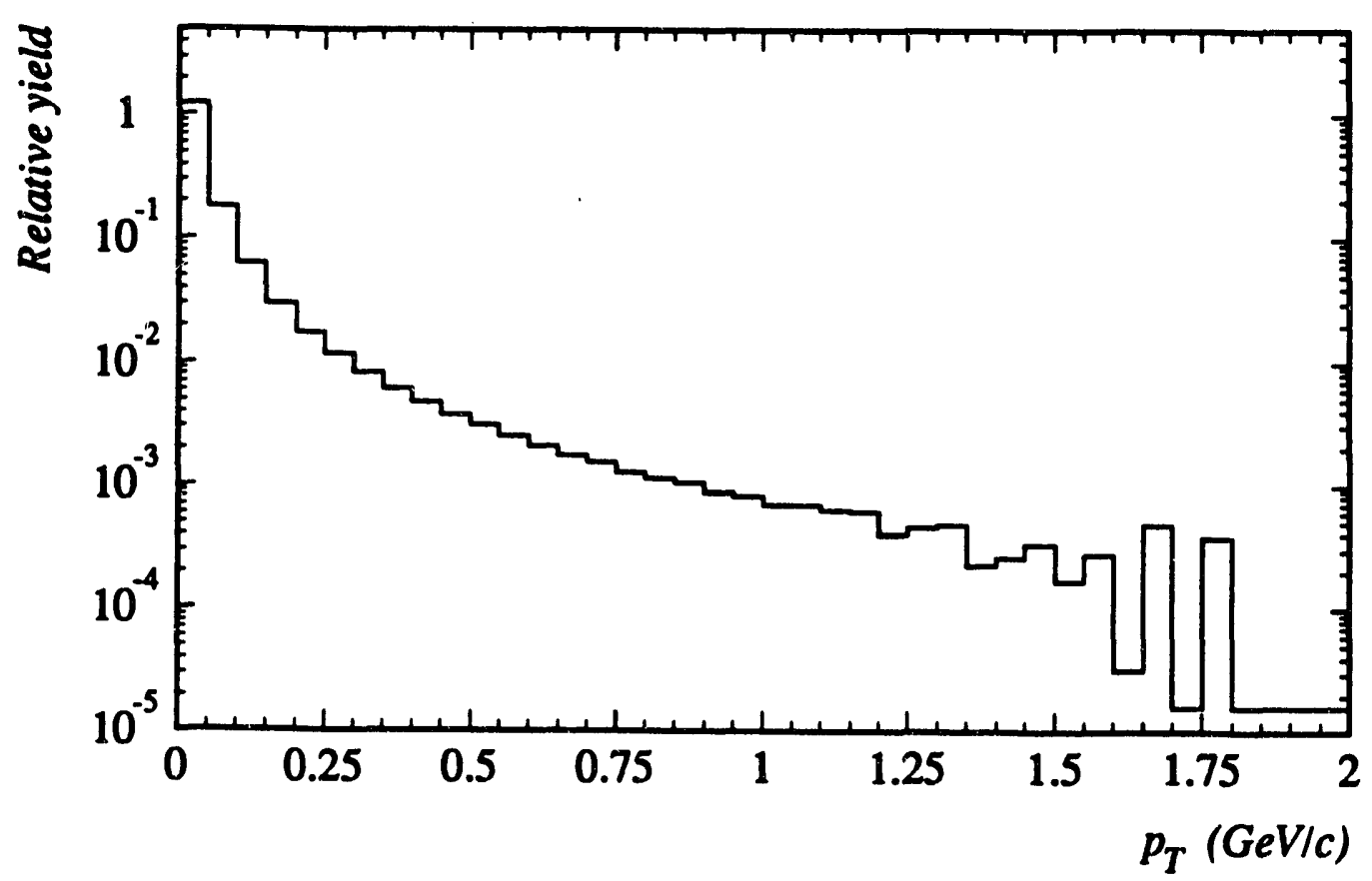

Figure 5.7: Ratio of the electron yield to the negative hadron yield as a function of $p_{T}$ for $0.0<y<4.0$. For $p_{T}>250 \mathrm{MeV} / c$ the electron contamination is less than $1 \%$. 


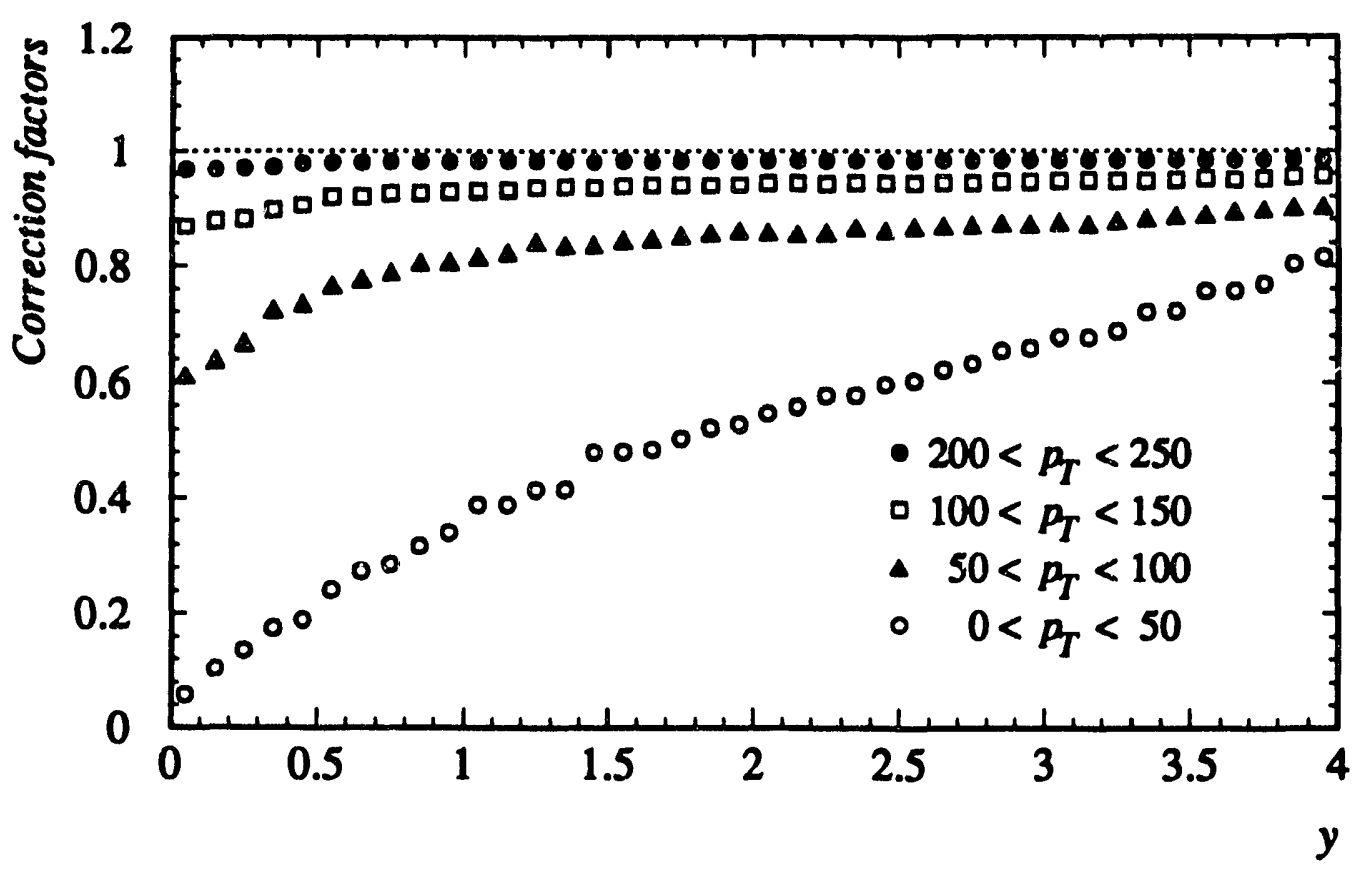

Figure 5.8: Representative elements of the electron correction table $c_{i j}\left(y, p_{T}\right)$. Each histogram plots the $c_{i j}$ dependence on $y$ for a different $p_{T}$ bin.

The systematic error in the electron correction will depend on the deviation of the FRITIOF input spectra from the real distributions which occur in nucleusnucleus collisions. A low- $p_{T}$ enhancement in the $\pi$ transverse momentum spectrum has not been established in high energy, symmetric $A A$ collisions. Since the sharp breaks observed in light projectile-heavy target $p_{T}$ spectra occur at $\sim 250 \mathrm{MeV} / \mathrm{c}$ [Str88], [Jac91a] the kinematics of the $\pi^{0}$ decay and $\gamma$ conversion process tend to push conversion $e^{-}$originating from the $p_{T}<250 \mathrm{MeV} / c$ region of the $\pi^{0}$ spectrum below $50 \mathrm{MeV} / c$ and out of our acceptance. The enhancement at high $p_{T}$ occurs where the cross section is down by two orders of magnitude or more. Since the FRITIOF $p_{T}$ distribution fits the data reasonably well in the heart of the spectrum the systematic error for the electron correction should be small.

\subsection{Non-vertex Particles}

\subsubsection{The simulation of $A A$ collisions including secondary production processes}

In order to investigate the influence of secondary charged particles on the charged particle spectra, the detector simulation package GEANT [Bru87] was employed to track particles through the NA35 experimental setup, to introduce secondary hadronic interactions in the target and detector material, and to properly decay short-lived stable particles, both kinematically and in coordinate space. The FRITIOF event generator combines a multiple collision model [And87] for the 
production of longitudinally excited color flux tubes-strings-with the Lund independent fragmentation model of hadronization [And83]. We used FRITIOF version 1.7 and the Lund Monte Carlo for Jet Fragmentation JETSET version 6.3.

Although the default set of FRITIOF/JETSET parameters have been tuned to predict the observed charged multiplicity, $p_{T}$ spectra, and $d N / d y$ distributions in pp collisions at the SPS and ISR energy scales, they fail to successfully predict the strangeness abundance reported by NA35 in ${ }^{32} \mathrm{~S}+{ }^{32} \mathrm{~S}$ collisions at $200 \mathrm{GeV} / \mathrm{c}$ [Bar90]. Moreover, certain fragmentation parameters reflect their $e^{+} e^{-}$heritage. For example, the parameter governing strangeness suppression is set to the value obtained for $e^{+} e^{-}$annihilation experiments rather than the value measured for lepton-hadron or hadron-hadron interactions [Ada87]. For these reasons, and because of the model dependence of an event generator-based correction, we have calculated the corrections discussed in this section with both the default set of parameters and with a modified parameter set which incorporates results from our experiment. The sensitivity of the correction to the parameter set will furnish an estimate of the systematic error. The pertinent parameters are listed in Table 5.1, denoted in the nomenclature of the FRITIOF source code. PAR(2), which accounts fo: the suppression of $s$ quark pair production compared to $u$ or $d$ pair produc-

\begin{tabular}{||l|c|c||}
\hline \hline Parameter & Default $^{4}$ & Modified \\
\hline PAR(2) & 0.30 & 0.60 \\
\hline PAR(12) & 0.35 & 0.60 \\
\hline PAR(31) & \multicolumn{3}{|c||}{0.60} \\
\hline PAR(32) & \multicolumn{2}{|c||}{1.20} \\
\hline PAR(52) & 1.00 & 10.0 \\
\hline \hline
\end{tabular}

Table 5.1: Default and modified FRITIOF/JETSET parameters. The role the parameters play in the Monte Carlo is discussed in the text.

tion, reflects the $\sim$ factor of 2 enhancement of the $\Lambda$ and $K_{S}^{0}$ yields measured by NA35 in central ${ }^{32} \mathrm{~S}+{ }^{32} \mathrm{~S}$ collisions. PAR(12) corresponds to the width of the Gaussian transverse momentum distribution assumed for the valence constituents of the primary hadrons. The modified value leads to a proton $p_{T}$ distribution with a mean transverse momentum $<p_{T}>\sim 600 \mathrm{MeV} / c$ near midrapidity consistent with preliminary results deduced from our chige-excess distributions. PAR(52) is essentially a stopping parameter which enhances the probability that in baryon fragmentation a leading baryon will appear closer to midrapidity. See Ref. And87 pg 120-125 and Ref. Sjo86 pg 380 for details. With the modification, the predicted proton rapidity distribution is in better agreement with our measured value [Wen90], also deduced from the charge-excess. PAR(31) and PAR(32) are the $a$ and $b$ parameters, respectively, in the Lund symmetric fragmentation function for the production of a hadron with transverse mass $m_{T}$ at position $z$ along the string

\footnotetext{
4The $a$ and $b$ fragmentation parameters, denoted by PAR(31) and PAR(32) respectively, differ in the context of FRITIOF 1.7 from the standard JETSET 6.3 values of $a=0.5, b=0.9$.
} 
axis,

$$
f(z)=z^{-1}(1-z)^{a} \exp \left(-b m_{T}^{2} / z\right) .
$$

They are explicitly shown for reference since these parameters have been widely modified by other members of the heavy-ion community to attempt to fit early results from both the AGS [Cos87] and CERN [Gyu87] programs.

The subset of FRITIOF final state particles input to GEANT consisted exclusively of particles(and their antiparticles) listed in the Stable Particle Summary Table of the 1988 Review of Particle Properties [PDG88]: the light mesons, the strange mesons, the nucleons and all strange baryons from the $\Lambda$ to the $\Omega^{-}$. Only particles directly produced by string fragmentation or by subsequent strong decays were retained as input, that is, all weak and electromagnetic decays were performed by GEANT during tracking. The weak decays of $\pi^{+}, \pi^{-}, \mathrm{K}^{+}$, and $\mathrm{K}^{-}$ were explicitly suppressed to avoid double counting. In practice, kink decays are detected visually in dedicated scans and overlooked during measuring programs devoted to vertex-based tracks (see the footnote on page 68 for a definition and some examples of kink decays).

The $E_{T}$ trigger derived from the PPD was not simulated. Events were selected by impact parameter $b$ by weighting according to $b d b$ for $0<b<b_{\max }=2.5 \mathrm{fm}$. This value of $b_{\max }$ achieved a good normalization of the Monte Carlo relative to the measured data, defined by consistency of three observables: the trigger crosssection $\sigma_{t r i g}$, the mean charged multiplicity in the three-view acceptance $\left\langle n_{3 V}\right\rangle$, and the number of participant protons. In the geometrical model, which assumes $\sigma(b)=$ constant so that the inelastic cross sections go like $\sigma=\int \sigma(b) 2 \pi b d b \propto b^{2}$,

$$
\sigma_{\text {trig }}=\frac{\sigma\left(b_{\max }\right)}{\sigma_{t o t}}=\left(\frac{b_{\max }}{2 R}\right)^{2} .
$$

For ${ }^{32} \mathrm{~S}$ nuclei with $R \simeq R_{0} A^{1 / 3} \simeq 3.7 \mathrm{fm}$, the Monte Carlo yields $\sigma_{\text {trig, } M C} \simeq 11 \%$ compared to the $\sigma_{\text {trig }}=.2$ barns $/ 1.8$ barns $=11 \%$ determined from run time scaler values for events which satisfied the PPD $E_{T}$ threshold [Hec90]. For both the matched and reconstructed measured data and the GEANT simulated data $\left\langle n_{3 V}\right\rangle_{\text {data }}=\left\langle n_{3 V}\right\rangle_{\text {sim }}=76.2$ charged particles. The number of participant protons is not a direct observable but must be inferred from the data. Moreover the participant number from the FRITIOF Monte Carlo is always greater than the observable number of participant protons in the final state $n_{p}-n_{\bar{p}}$ because of excitation of incident nucleons to hyperon states. From a fit to our measured proton rapidity distribution $d N / d y$, corrected according to the impact parameter selected simulation with $b_{\max }=2.5 \mathrm{fm}, \int_{-0.2}^{3.0}(d N / d y) d y=8.7$ protons. This is in reasonable agreement with the number determined from the simulation in the same rapidity domain, $\left\langle n_{p}-n_{\bar{p}}\right\rangle=9.3$. The inconsistency in predicted charged multiplicity of the optical simulations discussed in Section 4.5 was resolved by the full inclusion of non-vertex tracks.

From the point of view of GEANT, the streamer chamber plus target is an extremely simple detector setup. Since virtually all particles enter the chamber through a $120 \mu \mathrm{m}$ thick, mylar entrance window, the setup can be modelled as 
the $.57 \mathrm{~cm}$ thick, $1.2 \mathrm{~cm}$ radius cylindrical ${ }^{32} \mathrm{~S}$ target, a $72 \mathrm{~cm} \times 120 \mathrm{~cm} \times 120$ $\mu \mathrm{m}$ entrance plane, followed by the $72 \mathrm{~cm} \times 120 \mathrm{~cm} \times 2 \mathrm{~m}$ sensitive volume of the streamer chamber. The chamber is filled with an $80 \% \mathrm{Ne}, 20 \% \mathrm{He}$ gas mixture. The $\simeq 8 \mathrm{~cm}$ gap between the target and the mylar window is open to the atmosphere, that is, it is filled with air. The atomic weight $A$, nuclear charge $Z$, thickness $t$, and surface density $\rho t$ in units of $\mathrm{gm} / \mathrm{cm}^{2}$ of the target, window, air and chamber are shown in Table 5.2. A detailed discussion of the construction and operation

\begin{tabular}{||l|l|l|l|l||}
\hline \hline Material & $A$ & $Z$ & $t$ & $\rho t\left(\mathrm{gm} / \mathrm{cm}^{2}\right)$ \\
\hline S target & 32.066 & 16 & $0.57 \mathrm{~cm}$ & $0.590^{5}$ \\
Air & 14.61 & 7.30 & $8.00 \mathrm{~cm}$ & 0.00928 \\
Mylar window & 12.876 & 6.456 & $120 \mu \mathrm{m}$ & 0.0174 \\
Chamber gas & 18.913 & 9.374 & $2.00 \mathrm{~m}$ & 0.137 \\
\hline \hline
\end{tabular}

Table 5.2: Material parameters important for secondary hadronic interactions and $\gamma$ conversion. The $A, Z$ and $\rho$ for the mixtures/compounds are effective values calculated from the proportional weights of the molecular constituents.

of the streamer chamber can be found in Ref. Wen90. All other material is either outside of or downstream from the chamber and is unimportant for the calculation. The primary vertex for tracking primary hadrons was located at the center of the target for all events.

The GEANT version 3.14 parameters for the control of physical processes were set to include Gaussian multiple scattering, energy loss with Landau fluctuations, pair production, decay of unstable particles, and hadronic interaction with the generation of secondaries. The influence of multiple scattering and $d E / d x$ on the momentum resolution was imperceptible. The effect of multiple scattering on the vertex fit and $\chi_{v t x}^{2}$ distribution is discussed below. In this version of GEANT, secondary hadron-nucleus interactions in the target and detector material are calculated by invoking GHEISHA [Fes85], a code developed for the simulation of hadronic showers. GHEISHA relies heavily on the parametrization of data from $p p$ and $p A$ experiments. Approximately $90 \%$ of the charged secondaries result from inelastic $h A$ interactions, the remaining $10 \%$ from the absorption of $\pi^{-}$and $K^{-}$by heavy nuclei. Less than $.1 \%$ of the secondaries are primary particles which have participated in elastic or quasi-elastic scattering. The number of generated secondaries is calculated from a parametrization of $p p$ interactions which explicitly imposes charge, baryon number and strangeness conservation. Intranuclear cascading and nuclear evaporation of excited secondary target nuclei are included. Since the yield of secondary particles is dominated by $h A$ interactions induced by high energy ( $>10 \mathrm{GeV}$ ) primary hadrons which shatter the target nucleus and are accompanied by particle production, the known deficiencies of GHEISHA for handling slow particles should not be critical to our result [Wol91]. ${ }^{6}$

\footnotetext{
${ }^{5}$ Because the Monte Carlo events are positioned at the center of the target the effective thickness is $.57 / 2 \mathrm{~cm}$ for particles traversing the target along the beam direction.

${ }^{6}$ In the most recent versions of GEANT, GHEISHA has been supplemented, mainly to im-
} 
All final state, charged particles within the physics acceptance which both enter and exit the full streamer chamber sensitive volume are included in the calculation, with the exception of $e^{+}, e^{-}$which are treated separately as described in Section 5.3. They can be divided into three mutually exclusive categories, according to their process of birth:

1. Primary particles. In addition to the stable particles outlined above, charged particles from the electromagnetic decays of primary hadrons are included in this category. They cannot be discriminated in an inclusive measurement where the multiplicity in the final state is large. They are also included in the $p p$ data which we will compare to our results. The only significant electromagnetic contribution is from the charged decays of the $\eta$.

2. Decay particles. This class is comprised of the daughters from the weak decays of the abundant strange mesons and baryons.

3. Hadronic secondary particles. This class includes particles which have been elastically scattered resulting in a change in the detected momentum and particles participating in inelastic hadron-nucleus interactions. The final state particles from inelastic $h A$ secondary interactions divide conceptually into leading particles, produced particles and nuclear target fragmentation products.

Non-vertex tracks from weak decays and hadronic interactions will be referred to collectively as secondary particles. Bin-by-bin corrections to the single particle inclusive spectra can be computed from the ratio

$$
\frac{\text { primary }}{\text { primary }^{\prime} \text { decay }+ \text { hadronic }}
$$

for the appropriate inclusive distributions. The prime in the denominator means the subset of primary particles which have not been subject to decay or secondary interaction: the ratio in Equation 5.11 is what one hoped to measure divided by what one could(has) measure(d). Correcting with ratios, rather than by subtraction, compensates for inconsistencies between the Monte Carlo and the data, for example, in impact parameter selection and absolute normalization.

Figure 5.9 shows the number of charged secondaries per event, plotted logarithmically, as a function of secondary vertex position along the beam coordinate $x$ from $x=-120$ to $x=0 \mathrm{~cm}$, the midpoint of the streamer chamber. Particles originating in weak decays and secondary hadronic interactions are plotted separately. The shaded histogram is for weak decays. In spite of the particle-to-particle variations in the Lorentz boosts, the weak decay vertices in the laboratory are distributed approximately like the sum of two exponentials, corresponding to the

prove the simulation of low energy processes which dominate the termination of hadronic shower development. An independent calculation [Str91] for the NA35 detector setup has shown a strong dependence of the correction to the proton $d N / d y$ distribution on how the new code is invoked. Nevertheless, this preliminary calculation is in approximate quantitative agreement with our result. 


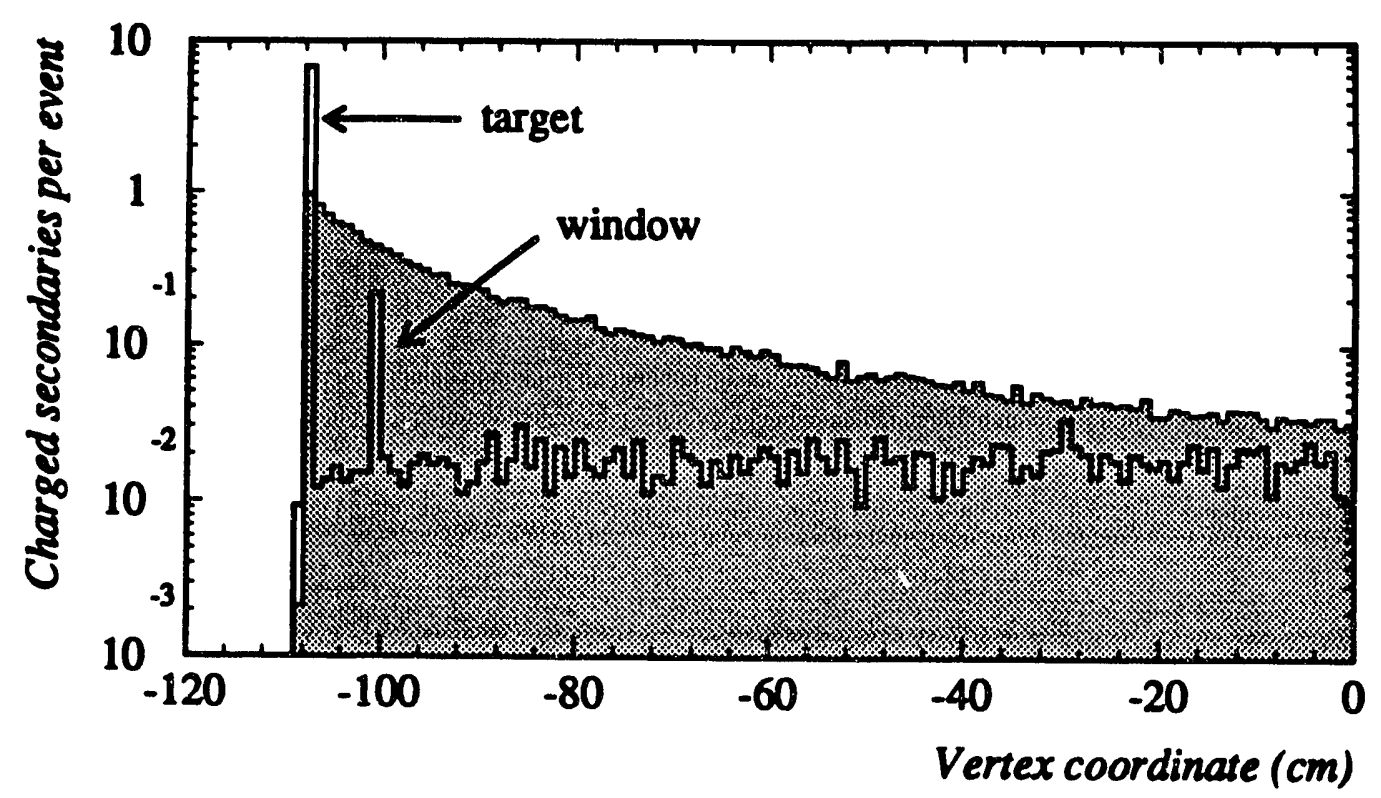

Figure 5.9: Number of charged secondaries as a function of secondary vertex position along the beam coordinate. Secondaries from weak decays(shaded region) and hadronic interactions(dark solid line with peaks at the target and window) are plotted separately. In the coordinate system shown the $\mathbf{2} \mathrm{m}$ streamer chamber extends to $\pm 100 \mathrm{~cm}$.

mean lifetimes of the $\Lambda$ and $K_{S}^{0}$. For the default parameter set, FRITIOF predicts relative yields from $K_{S}^{0}, \Lambda$, and charged $\Sigma$ decays of $60 \%, 30.9 \%$ and $8.3 \%$, respectively, with very small contributions from the $\Xi$ and $\Omega$. The hadronic secondary distribution has sharp peaks at the positions of the target and entrance window and a flat background from the air and chamber gas. The ratio of the yields at the peaks is $\simeq(\rho t)_{\text {target }} /(\rho t)_{\text {window }}$, as one would expect. Approximately $65 \%$ of the hadronic secondary yield arises from the target, the entrance window and air upstream of the streamer chamber sensitive volume. Although the integrated yield out to $x=0$ of secondaries from decay is about $70 \%$ greater than the yield from hadronic interaction, after a cut on $x$ which corresponds to the $\chi_{v t x}^{2}$ cut made on the data the integrated yield from hadronic secondaries is comparable to that from weak decays for the default parameter set. Excluding charged particles from electromagnetic decays but including electrons from $\gamma$ conversion $\simeq 20 \%$ of the total charged multiplicity has a vertex of origin different from the primary vertex. This is in reasonable agreement with the fraction of reconstructed particles in the measured data whose $\chi_{v t x}^{2}$ is inconsistent with the expected exponential distribution $f\left(\chi^{2}, \nu=2\right) \propto \exp \left(-b \chi^{2}\right)$. See page 112 and Figure 4.25.

Secondaries from decay and hadronic processes are generally not visually identifiable. This is made clear in Figure 5.10 which displays the streamer chamber trajectories, projected into the bend plane, of all charged secondaries in a typical event. There are no primary tracks in this figure. Only secondaries which have a non-negligible probability of being discriminated, either visually or using infor- 


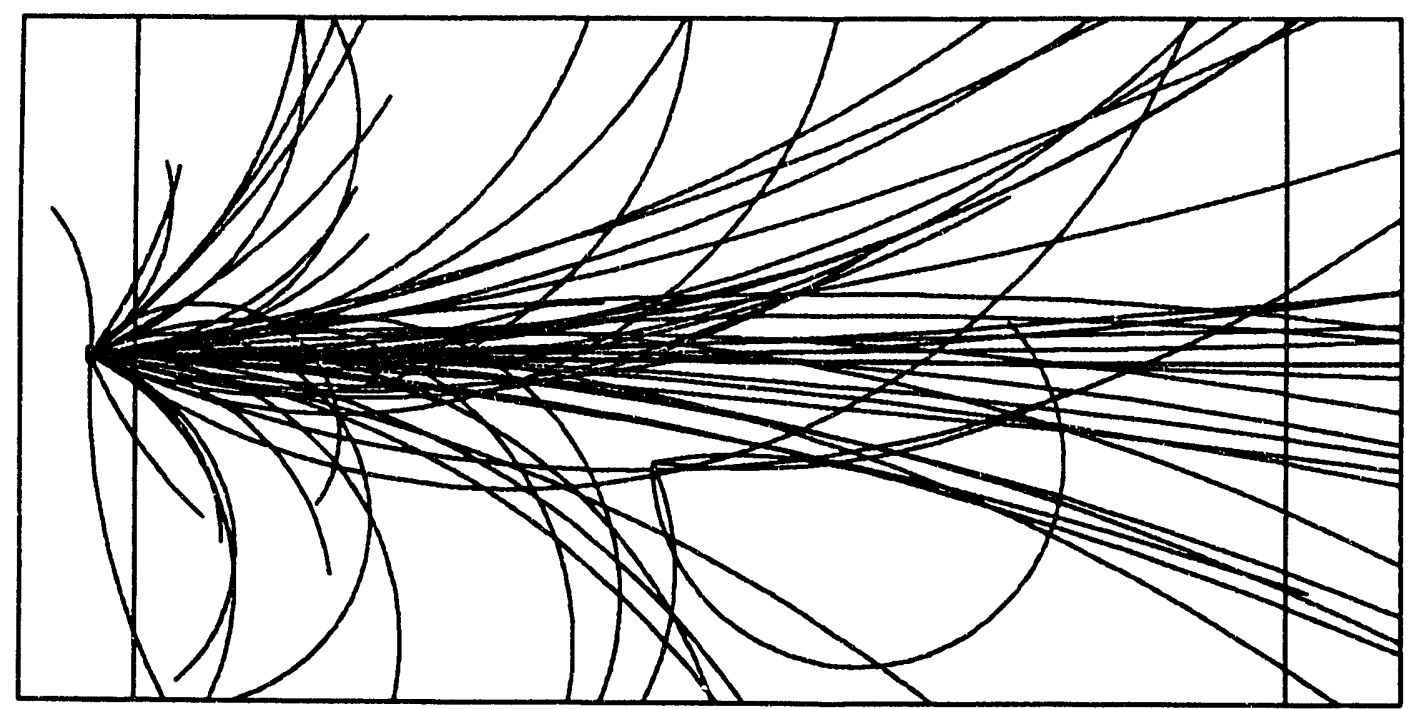

Figure 5.10: Bend-plane trajectories of charged secondaries for one event. Only particles from the obvious gas interaction, in the lower middle of the figure, would be identified during track measuring and rejected.

mation returned from event reconstruction, are plotted, that is, $e^{+}, e^{-}$from Dalitz decays and charged hadrons from electromagnetic decays have been omitted from the figure. $V^{0}$ decays for which the decay vertex is unobscured are rare, even if the decay is far downstream from the target. For the NA35 neutral strange particle analysis, this resulted in severe fiducial volume cuts, large inversion weights for the detected particles, and severe low-p phase space cutoffs [Bam89]. For the complete event analysis, secondary particles had to have been measured as if they were vertex tracks, almost without exception.

Secondaries from decay and hadronic processes differ dramatically in kinematic character. Figure 5.11 shows two-dimensional histograms, represented as box plots, of the number of negatively charged hadrons as a function of rapidity and $p_{T}$. The size of the box is proportional to the number of particles in the bin. Rapidity is calculated assuming the pion mass. The figure for primary hadrons exhibits the expected features of a superposition of soft $N N$ collisions at $\sqrt{s}=20 \mathrm{GeV} / c$ : $<p_{T}>\simeq 350 \mathrm{MeV} / c$, yield increasing with rapidity, and a plateau in the particle production which stretches for $\sim 1$ unit of rapidity down from midrapidity. Charged hadrons from weak decay are concentrated at much lower $p_{T}$, are peaked at $y \simeq 1.5$ rather than near midrapidity, and are unlikely to introduce a high$p_{T}$ particle into the tail of a $p_{T}$ distribution. These features are typical of the folding of decay kinematics onto a primary spectrum [Bro91]. Both primary and weak decay phase space distributions for negative hadrons are symmetric about midrapidity-the distributions in the forward hemisphere in the center of mass for $y>3.0$ are the same.

The main features of the figure for hadronic secondaries-the presence of a high- 

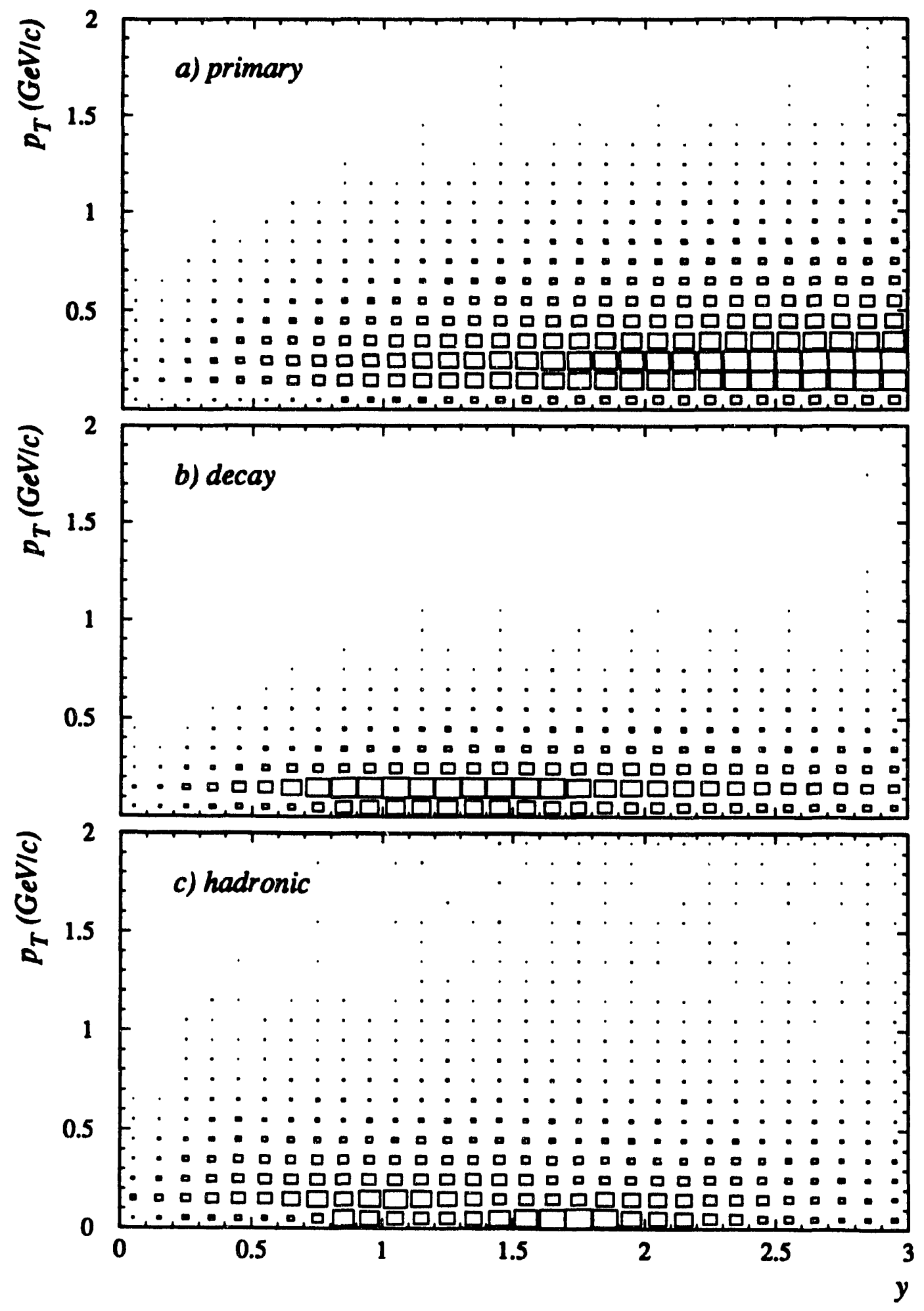

Figure 5.11: Number of negative hadrons as a function of $\left(y, p_{T}\right)$ phase space for particles originating in a) primary, b) decay and c) secondary hadronic processes. 
$p_{T}$ component over virtually the full rapidity range, an enhanced low- $p_{T}$ component at rapidities intermediate between the target and midrapidity, and a broad distribution in rapidity centered at $y \sim 1.5$ which falls rapidly approaching midrapiditycan be understood qualitatively as the superposition of a leading particle effect and shifts in phase space of both the available energy and the effective center of mass. Relatively high $p_{T}$ primary hadrons, which can serve as the "beams" for secondary interactions, can be rescattered in average $h A$ collisions into the tail of the $p_{T}$ distribution, $p_{T}>1 \mathrm{GeV} / c$, over a wide range of rapidity. Similarly, the $p_{T}$ of secondary particles produced in average $h A$ collisions refers to a collision axis which may be inclined at a relatively large angle in the laboratory, leading to an apparent high $p_{T}$. The large yields at low rapidities exhibit an enhancement at low $p_{T}$ relative to the $p_{T}$ distribution for primary, produced hadrons. The $\left\langle p_{T}\right\rangle$ for $\pi^{-}$produced in $p p$ collisions falls from $\sim 350 \mathrm{MeV} / c$ at $p_{l a b}=200 \mathrm{GeV} / c$ $(\sqrt{s}=20)$ to $\sim 275 \mathrm{MeV} / c$ at $p_{\text {lab }}=5 \mathrm{GeV} / c$ while the spectral shape becomes more peaked at low $p_{T}$ [Bog74]. In the present context, this can be loosely attributed to phase space although the dynamical origins of the trend are of course more complicated. The $\left(y, p_{T}\right)$ distribution for negative hadrons from hadronic secondary interactions is not symmetric about midrapidity. Rather, its characteristic features occur on a rapidity scale typical of a projectile-target system with $<y_{\text {proj }}>\sim 3.0$. Only $\simeq 10 \%$ of the negatively charged hadronic secondaries overflow Figure 5.11c with $y>3.0$ compared to $\sim 1 / 2$ of those from weak decay. The rapidity dependence of hadronic secondaries diminishes their impact on the low $p_{T}$ part of the spectrum for $y>2.0$.

Nuclear target fragmentation is important for the proton spectra. This is illustrated in Figure 5.12 which plots the bend-plane trajectories of the charged particles in one event resulting from secondary hadronic interactions in the target. Because they arise from $h A$ collisions, they introduce a false charge-excess, carried by target protons and light fragments. Because the incident "beams", on average, have significantly lower momenta and because the rapidity shifts of interacting target nucleons in $h A$ interactions are small [To087], this effect is most pronounced at rapidities in the target fragmentation region.

Because the yield of secondaries is so large, and because the secondary vertices are for the most part not visually discernible, a modest sample of simulated CCD streamer chamber images was constructed and processed through the complete data reduction chain to address three questions:

1. What was the efficiency for reconstructing non-vertex tracks under the mistaken assumption that they emanated from the vertex? Would failure of the momentum reconstruction help suppress them?

2. Could the non-vertex tracks bias the vertex fit and account for the broadening of the $\chi_{v t x}^{2}$ distribution discussed in Section 4.7, resulting in a slope of the exponential core of the distribution which was much less steep than the ideal value of $b=-0.5$ ? Could multiple scattering partially account for the broadening? 


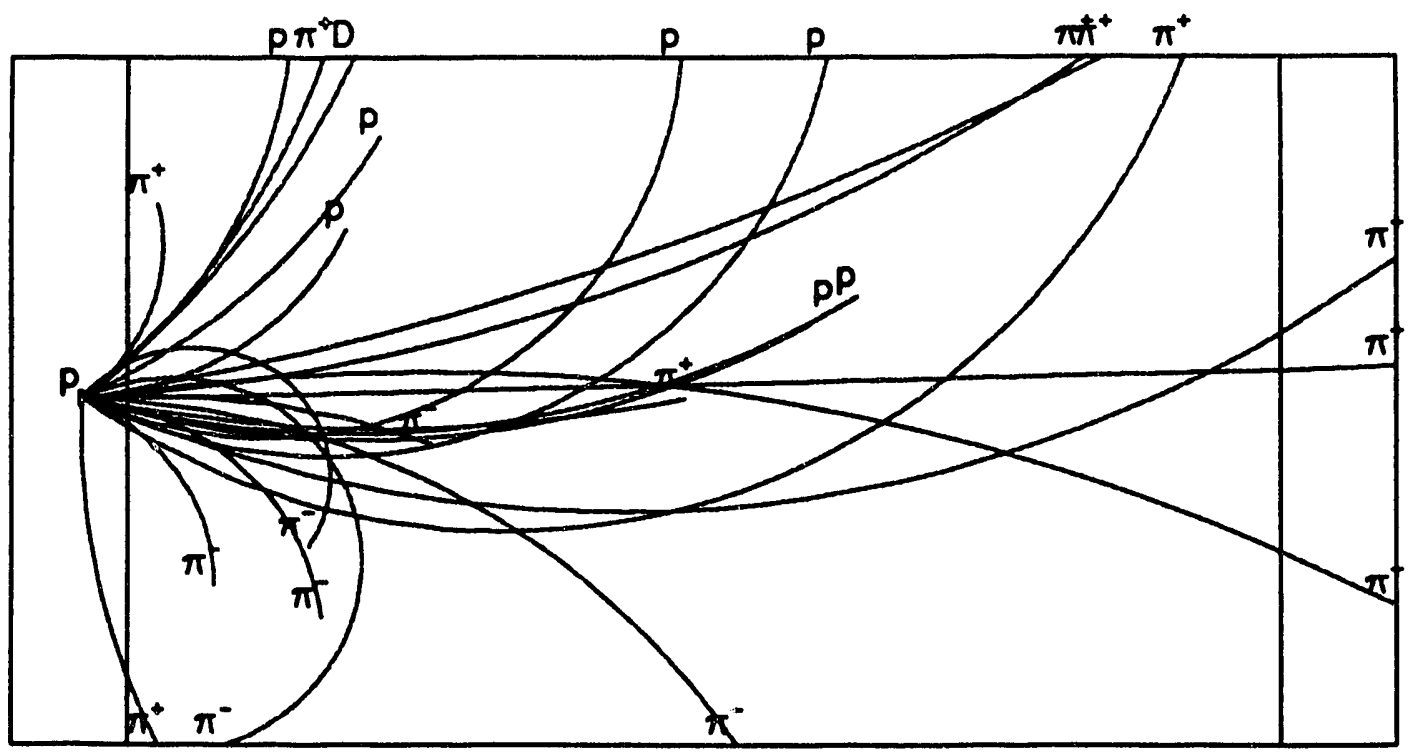

Figure 5.12: Bend-plane trajectories of secondaries produced in hadronic interactions. Note the positive charge-excess carried by the protons $(p)$ and light nuclear fragments $(D=$ deuteron) from nuclear target fragmentation.

3. For non-vertex tracks which successfully reconstructed, how efficient was the $\chi_{v t x}^{2}$ cut at rejecting them from the analysis?

The CCD image sample retained all charged particles, including all secondaries, in the three-view acceptance.

The reconstruction efficiency for non-vertex tracks is $\simeq 98 \%$, in spite of the fact that more than $20 \%$ of the secondary vertices occur within the streamer chamber sensitive volume, more than $\sim 10 \mathrm{~cm}$ from the primary vertex employed for the momentum reconstruction. Secondary particles, originating from decay vertices buried in the forward cone, can be imaged in the CCD three-view acceptance and reconstructed successfully which have vertex coordinate $x$ as far as $50 \mathrm{~cm}$ downstream from the streamer chamber entrance window. Non-vertex tracks are not suppressed by failure of the momentum reconstruction.

There was no evidence that either multiple scattering or the inclusion of secondary particles in the vertex fit biased the position of the fitted vertex. The fitted vertex coordinates deviated from the Monte Carlo input by less than $700 \mu \mathrm{m}$ with the beam coordinate slightly skewed downstream of the target. This resolution had a negligible impact on reconstructed momenta and track selection based on the $\chi_{v t x}^{2}$ cut. Moreover, the $\chi_{v t x}^{2}$ distribution, although acquiring the anticipated tail from non-vertex tracks, was well fitted by an exponential with only a small decrease in slope from $b=-0.5$. To further explore possible causes for the broadening of the $\chi_{v t x}^{2}$ distribution of the measured data we subverted the iterative fit by assigning an unrealistic value to the iteration cutoff parameter, which forced all 
secondaries to be included in the vertex fit. Although this could bias the vertex position by as much as $5 \mathrm{~mm}$, it could not dramatically broaden the $\chi_{\text {vtx }}^{2}$ distribution. This exercise confirmed that the vertex fits for the measured data employed a reasonable value for the iteration cutoff parameter and converged to stable solutions. We speculate that the broadness of the measured $\chi_{v t x}^{2}$ distribution is due to a physical effect in the chamber which occurs during the primary ionization and streamer development phase, perhaps the prepulse which drifts all the ionized charge along the magnetic field direction before the application of the primary high-voltage pulse. ${ }^{\text {? }}$

For the simulated and reconstructed CCD image sample we can define a $\chi_{v t x}^{2}$ cut which has an unambiguous meaning. For an exponential distribution $f(x)=$ $A \exp (-b x)$, a value of the upper integration limit $x_{\max }$ can be defined so that the integral from 0 to $x_{\max }$ is a fraction $c$ of the total integral from 0 to $\infty$,

$$
\int_{0}^{x_{\text {max }}} f(x) d x=c \int_{0}^{\infty} f(x) d x .
$$

For given values $(b, c)$

$$
x_{\max }(b, c)=\frac{\ln (1-c)}{-b} .
$$

Since $f(x)$ above is the form of the $\chi^{2}$ probability density function $f\left(\chi^{2}, \nu=2\right)$, Equation 5.12 implies that the probability of finding a vertex track with $\chi^{2}>\chi_{\max }^{2}$ is $(1-c)$. From an exponential fit to the simulated CCD data $\chi_{\max }^{2}(c=0.999) \simeq 15$. A cut at this value actually rejects $.3 \%$ rather than $.1 \%$ of the primary vertex tracks in simulated data. The non-vertex track rejection efficiency of the $\chi_{v t x}^{2}$ cut follows at once by applying it to the secondary charged particles. The efficiency of the cut is only $\simeq 17 \%$. However, the correlation between $\chi_{u t x}^{2}$ and distance of the secondary vertices from the primary vertex is strong, a fact we exploit below. Since our simulation was unable to reproduce the scale of the $\chi_{v t x}^{2}$ distribution the cut applied to the measured data was defined in an analogous way from the fit to the exponential core, $\chi_{\text {data }}^{2}=\chi_{\text {max }}^{2}\left(b_{\text {data }}, c=0.999\right)$. See Figure 4.25 .

It was impracticable to generate enough CCD images to calculate a statistically significant secondary correction by processing large numbers of events through the full data reduction chain and applying cuts identical(or equivalent) to those made to the data. In order to approximately incorporate the effect of the $\chi_{v t x}^{2}$ cut, the corrections only include secondary particles whose vertex of origin along the beam direction $x$ was upstream of a position cut which was determined from the correspondence between $x$ and $\chi_{v t x}^{2}$. The correspondence was not one-to-one which means that some charged particles excluded from the data by the $\chi_{v t x}^{2}$ cut were included in the correction and vice-versa. However, this inconsistency affected only $\simeq 7 \%$ of the particles which figured in the calculation. The cut required $x<-99.0 \mathrm{~cm}$ and was constructed to have the same rejection efficiency as the cut $\chi_{v t x}^{2}<\chi_{\max }^{2}$. Since the cut is just inside the streamer chamber sensitive volume

${ }^{7}$ The prepulse was $\sim 300 \mathrm{~ns}$ wide and $\sim 2 \mathrm{kV}$ in amplitude. It was opposite in sign from the primary HV pulse, resulting in a shift in direction of the electric field in all three regions of the chamber and a $2 \mathrm{~mm}$ displacement in the $z$ coordinate of the fitted vertex [San91]. 
all hadronic secondary interactions which occurred in the mylar entrance window were included in the calculation. Virtually all gas interactions were excluded. Because the cut is close to the primary vertex it will help minimize momentum shifts which may occur to reconstructed secondaries but which were neglected in our high statistics calculation. Corrections to the negative hadron $p_{T}$ spectra and $d N / d y$ distribution were computed for the full phase space acceptance, and for the rapidity intervals $1.0<y<2.0$, and $2.0<y<2.8$. The correction to the proton $d N / d y$ distribution was calculated for a number of different assumptions for the proton acceptance including the most restrictive one $p_{T}>200 \mathrm{MeV} / c$ and $0.6<y_{p}\left(m_{\pi}, p_{T}, p_{L}\right)<2.8$.

\subsubsection{Corrections to the negative hadron $p_{T}$ and $d N / d y$ distributions}

The contributions of the primary, weak, and secondary hadronic processes to the negative hadron transverse momentum spectrum $1 / p_{T} d N / d p_{T}$ as a function of $p_{T}$ are shown in Figure 5.13 for the modified parameter set. The distributions plotted

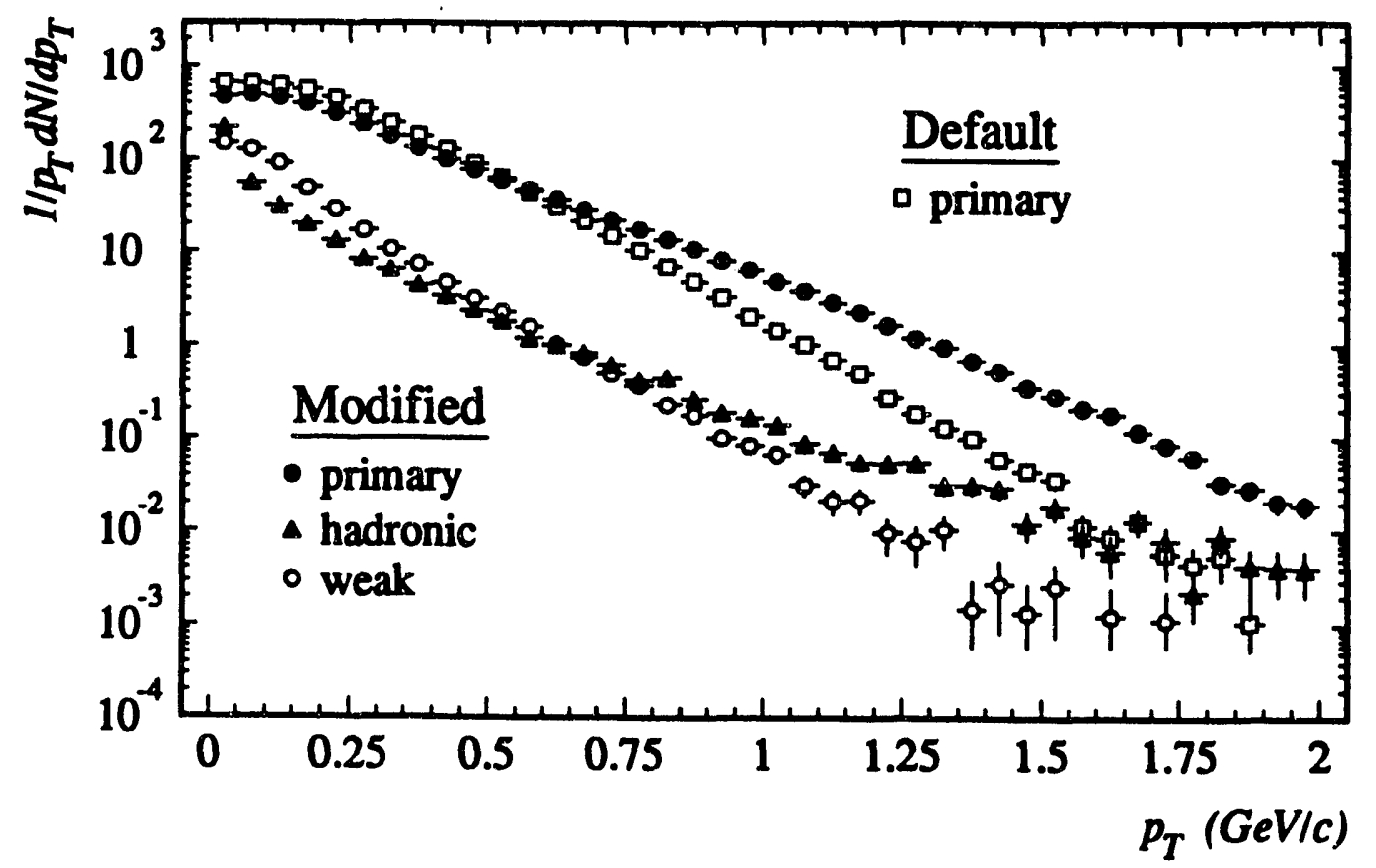

Figure 5.13: Contributions of primary, weak and secondary hadronic processes to the transverse momentum distribution. The distribution for the primary class is shown for both the default and modified parameter set.

in Figure 5.11 , weighted by $1 / p_{T}$, are integrated over the full physics rapidity acceptance $0.6<y<2.8$ to obtain the single particle inclusive spectra for the three classes. The histograms are normalized to the total negative charged multiplicity. Also shown, indicated by the open boxes, is the primary spectrum for the default parameter set. The hadronic secondary $p_{T}$ spectrum has a convex shape featuring 
enhancements at low and high $p_{T}$ of the kind which has stimulated a great deal of theoretical investigation. The yield at high $p_{T}$ exceeds that of the primary spectrum for the default parameter set. The sum of the weak and hadronic effects is $\sim 1 / 2$ of the total observable yield in the first $p_{T}$ bin. Note the dramatic difference in the primary $p_{T}$ spectra for the different parameter sets for $p_{T}$ greater than $\sim 0.8 \mathrm{GeV} / c$. The hadronic secondary distribution, dominated by material and kinematics, is independent of the FRITIOF input parameters. The shape of the weak distribution is approximately independent of the FRITIOF input parameters although the yield for the modified set increases by $\sim$ a factor of two, consistent with the relaxation of the strangeness suppression factor.

We correct our data using ratios calculated from results of the Monte Carlo with the modified parameter set. The justification for this is shown in Figure 5.14, which compares the negative hadron $d N / d p_{T}$ spectra of the full Monte Carlo to

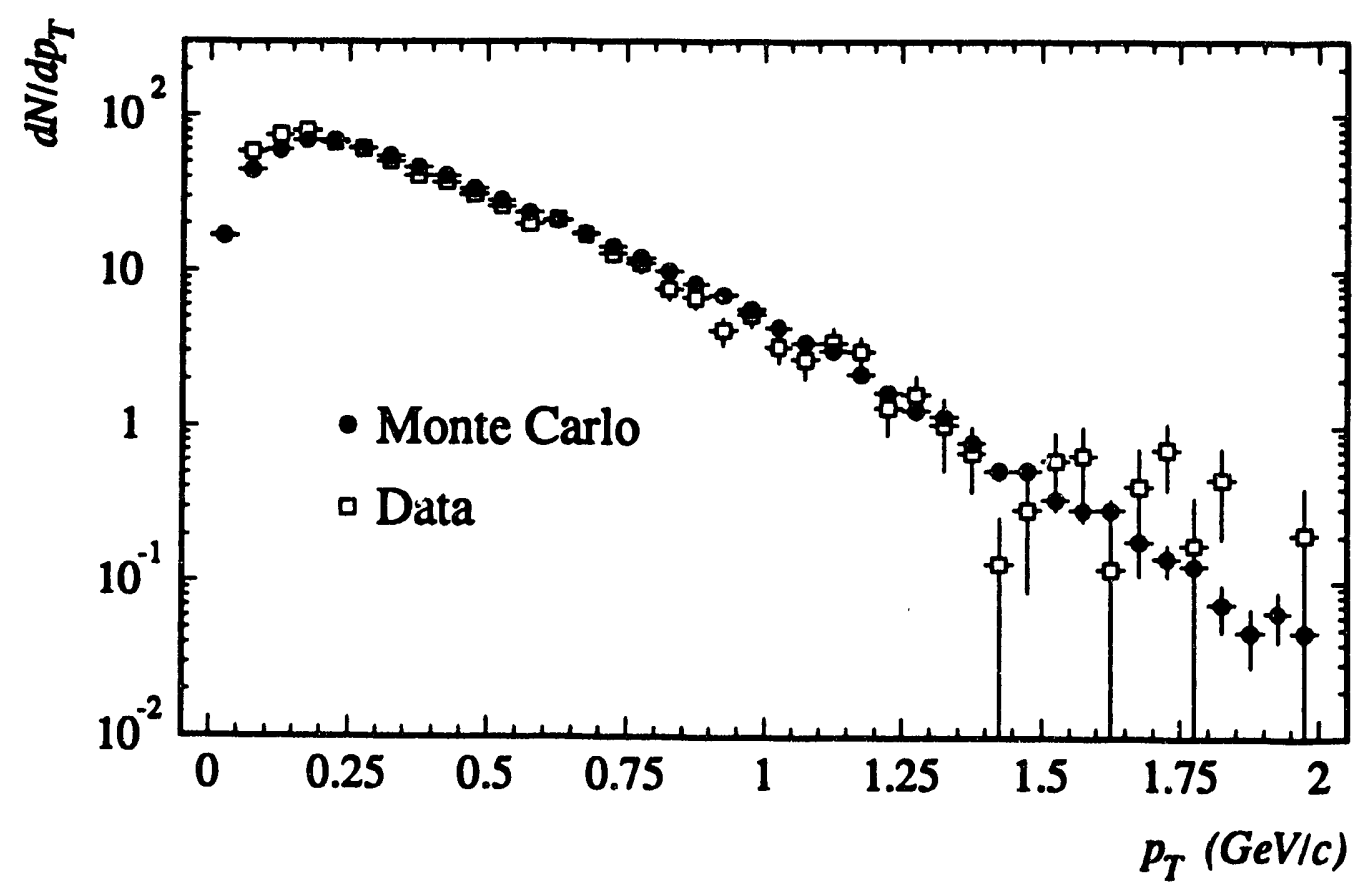

Figure 5.14: Comparison of negative hadron $d N / d p_{T}$ spectra of measured data and the full Monte Carlo. The measured data have been electron-corrected and inverted with the geometrical weights.

the measured data. The distribution for the data has been efficiency-corrected by inverting the raw histogram with the track-by-track geometrical weights and electron-corrected by the method discussed in Section 5.3. It has not yet been corrected for charged secondaries. The simulated distribution includes all negative hadrons, from all three processes of birth. Electrons have been omitted. The measured data have no data point in the first bin for $p_{T}<50 \mathrm{MeV} / c$. The agreement between the simulation and the measurements is exceptional both in shape and scale, except for a small discrepancy for $p_{T}<200 \mathrm{MeV} / c$. This is further evidence for the good normalization of the Monte Carlo event selection to 
the data. The close agreement of the spectra, in conjunction with the kinematic arguments presented earlier, also suggests that the systematic error in the electron correction is negligible.

The ratio of the observable negative hadron $p_{T}$ spectrum to the primary spectrum and its inverse, the correction factors of Equation 5.11, are shown for both parameter sets in Figures 5.15 and 5.16. The large values and statistical errors of the ratio for the default parameter set for $p_{T}>1.5 \mathrm{GeV} / c$ result from the rapid falloff of the primary distribution at high $p_{T}$. Note that the ratio/correction factors are larger for the modified parameter set at low $p_{T}$. This is a consequence of the increased yield from weak decays of neutral strange particles, the increased width of the Gaussian distribution assumed for the generation of transverse momentum, and the somewhat counter intuitive result that increasing the stopping power decreases the particle production. The dashed lines in Figure 5.16 are quadratic fits for $p_{T}>1.0 \mathrm{GeV} / c$ which smooth the correction. For $p_{T}<1.0 \mathrm{GeV} / c$ the systematic error in the correction can be estimated from the difference in the correction factors for the two different input parameter sets. For the lowest three $p_{T}$ bins the systematic errors are $\sim 20,10$, and $9.5 \%$ and quickly fall to less than $5 \%$. For $p_{T}>1.0 \mathrm{GeV} / c$, where the difference in the primary spectra leads to a dramatic difference in the correction factors, we rely on the agreement between the measured data and the simulation with the modified input parameter set shown in Figure 5.14. A change by a factor of 2 in the contribution of the weak plus hadronic yield would still lead to systematic errors less than $\sim 12 \%$ at high $p_{T}$.

We explored the rapidity dependence of the correction by calculating it for three different rapidity intervals: the full acceptance, near target rapidity $1.0<y<2.0$, and at midrapidity $2.0<y<2.8$. The results for $p_{T}<1.0 \mathrm{GeV} / c$ are shown in Figure 5.17. The correction factors(ratios) are less than(greater than) the dotted line drawn at unity. The role of secondaries is most pronounced for $1.0<y<2.0$, the rapidity interval which includes the peaks in the negative hadron yields from hadronic secondary interaction and weak decay, and where the primary particle production is still rising fairly rapidly before the onset of the central plateau. For $p_{T}>1.0 \mathrm{GeV} / c$ (not shown), there is no dependence on rapidity within statistical errors. At least an order of magnitude naore than the 20,000 simulated events employed for the results shown here would be needed to study the rapidity dependence more carefully at high $p_{T}$. Because of the large statistical errors of the measured data at high $p_{T}$ there was no motivation for a higher statistics study. The high- $p_{T}$ quadratic fit to the correction factors for $0.6<y<2.8$ was used to correct the transverse momentum spectra in all three rapidity intervals.

The correction to the negative hadron $d N / d y$ distribution is shown in Figure 5.18. From the difference in the correction factors for the two parameter sets we estimate the systematic error is less than $\sim 6 \%$ for all rapidities, falling to less than $2 \%$ at midrapidity. This error is of the same size as the inflection in Figure 5.18 for $y \simeq 0.7$ which may be artificial. It results from a shoulder in the $d N / d y$ distribution for hadronic secondaries which may arise from a GHEISHA parametrization of hadron-nucleus data with a large systematic error or a known bias in the pseudorapidity distribution for shower particles. See Ref. Fes85 pages 


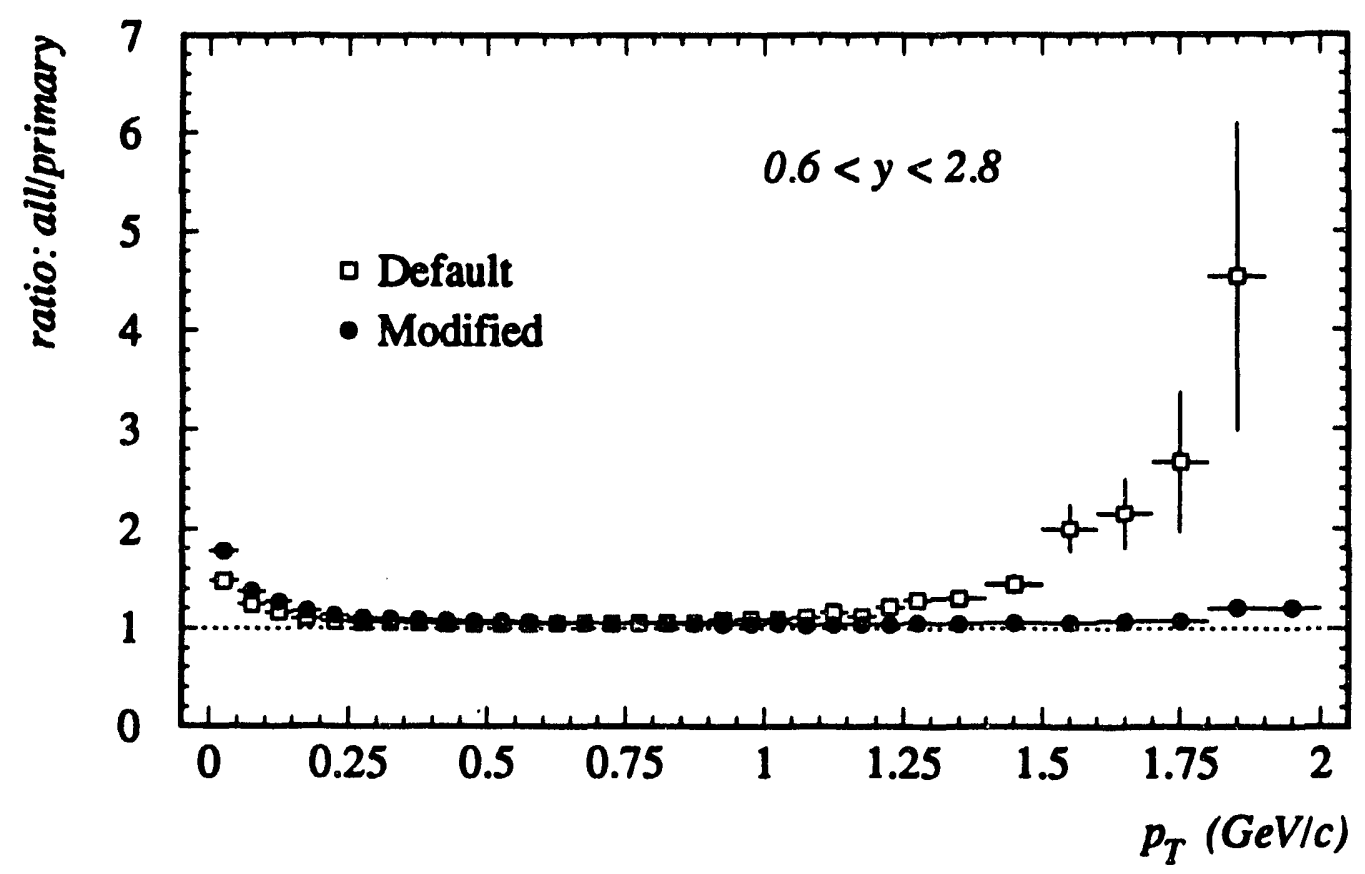

Figure 5.15: Ratio of observable spectrum-the sum of the primary, weak and secondary hadronic components-to the primary spectrum as a function of $p_{T}$.

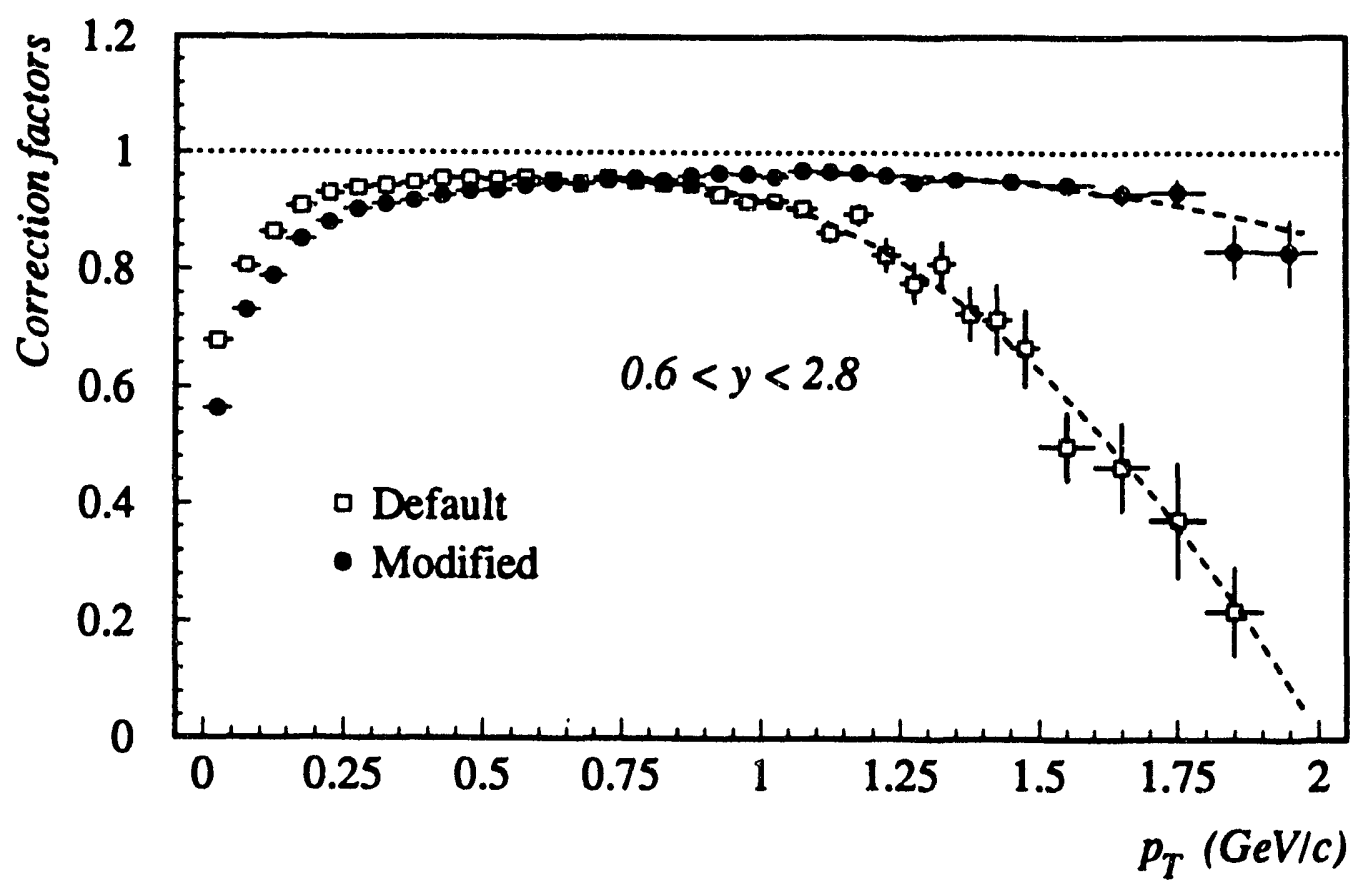

Figure 5.16: Correction factors to the negative hadron $p_{T}$ spectrum. The dashed lines are quadratic fits for $p_{T}>1.0 \mathrm{GeV} / c$. 


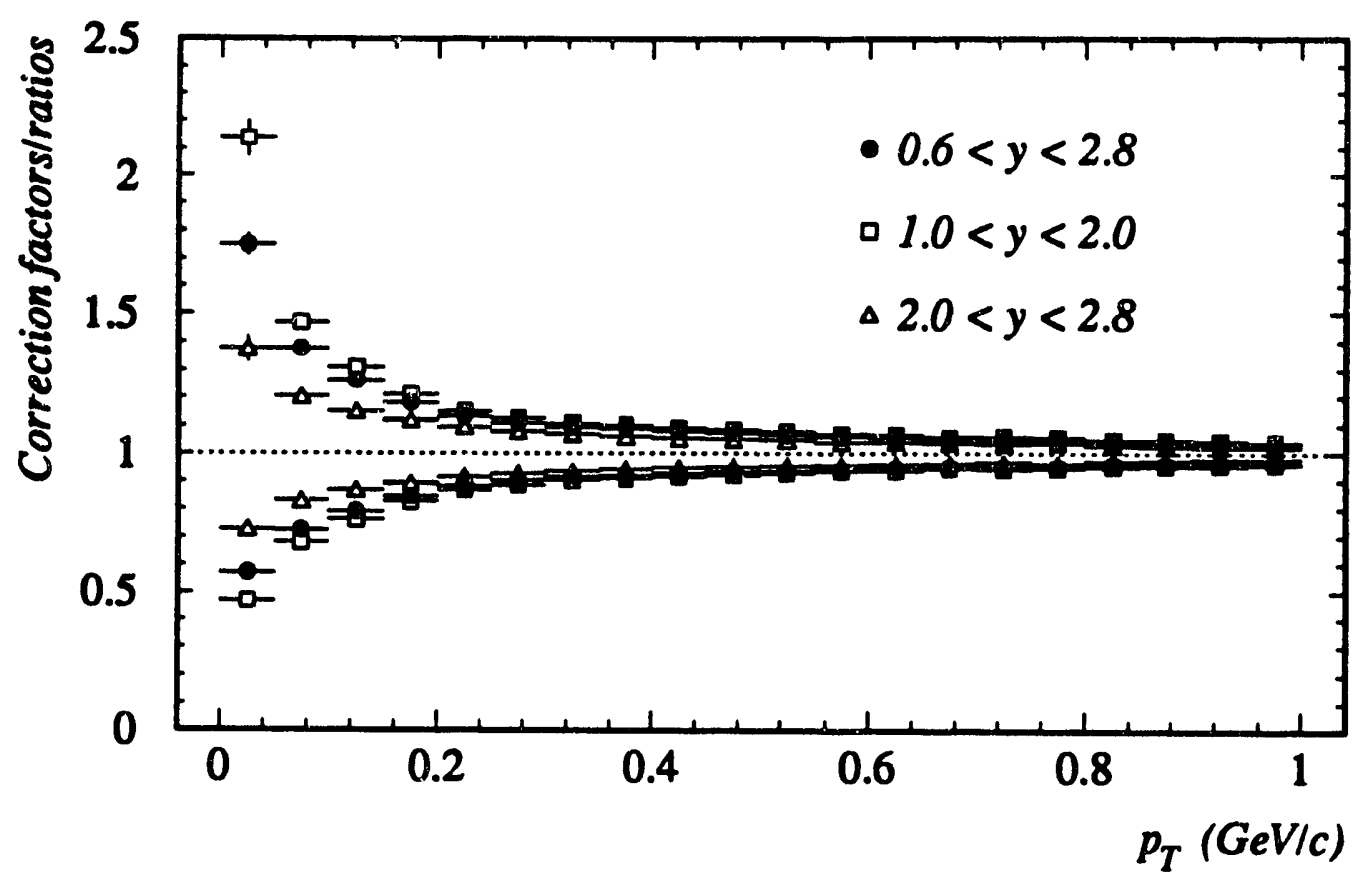

Figure 5.17: Rapidity dependence of the ratio and correction factors to the negative hadron $p_{T}$ spectrum. For $p_{T}>1.0 \mathrm{GeV} / c$ the dependence is much weaker.

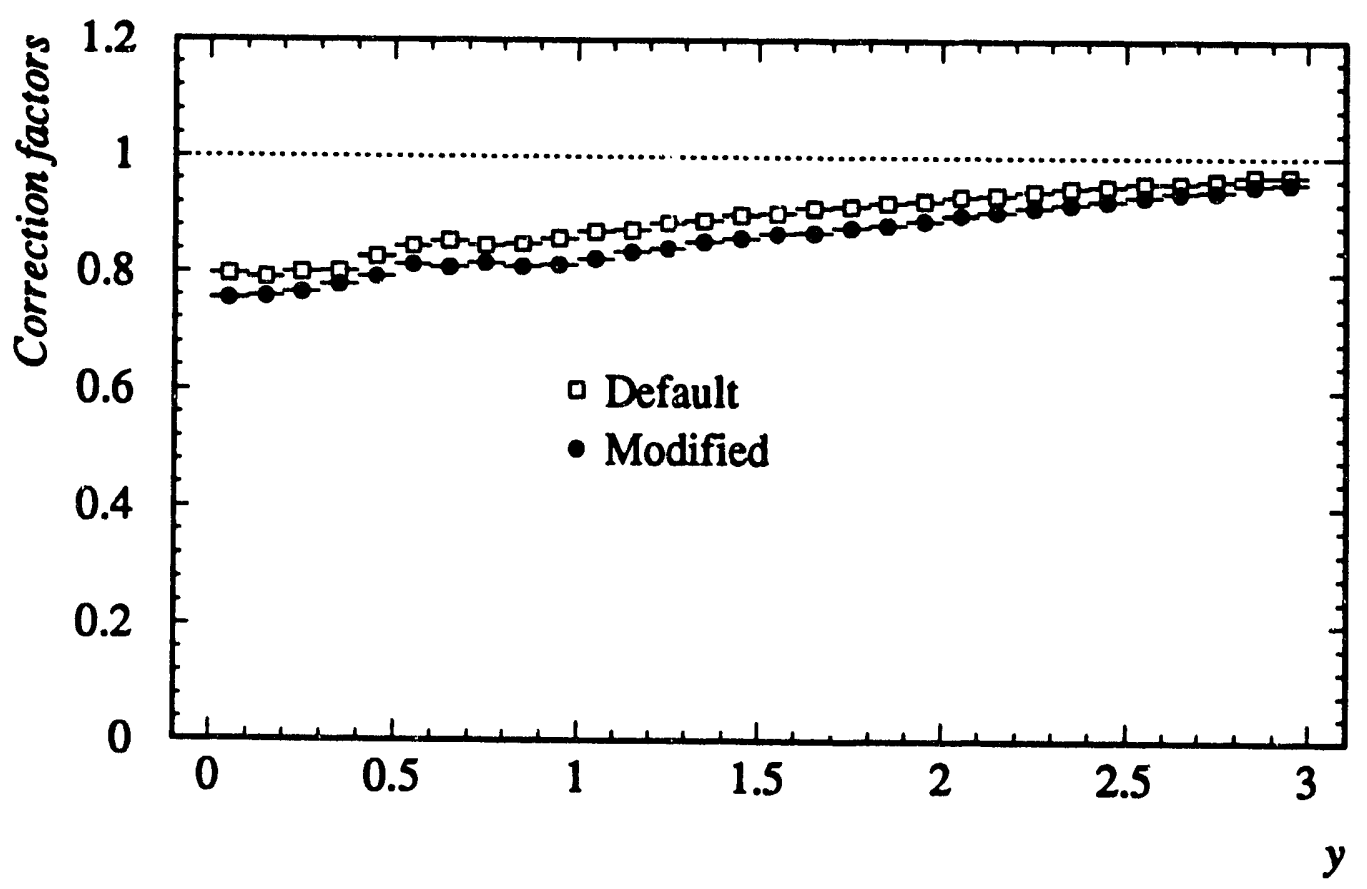

Figure 5.18: Correction factors to the negative hadron $d N / d y$ distribution. 
$131-146$.

\subsubsection{Corrections to the proton $d N / d y$ and $p_{T}$ distributions}

The observable charge-excess distributions for rapidity and transverse momentum are essentially the sum of four contributions: the incident baryons ${ }^{8}$ we are trying to measure $(p-\bar{p})$, the charged kaon excess $\left(K^{+}-K^{-}\right)$from associated production-like channels, the hadronic secondary excess $\left(h^{+}-h^{-}\right)_{h a d}$ from nuclear target fragmentation, and the excess from weak decays $\left(h^{+}-h^{-}\right)_{\text {weak }}$. The weak excess can be either positive or negative depending on rapidity and the low $p_{T}$ acceptance cutoff. This decomposition is illustrated in Table 5.3, which tabulates the $d N / d y$ distributions for the four cases outlined above, their sum, and the distribution one can

\begin{tabular}{||c|c|c|c|c|c|c||}
\hline \hline$y$ & $p-\bar{p}$ & $K^{+}-K^{-}$ & $\left(h^{+}-h^{-}\right)_{\text {had }}$ & $\left(h^{+}-h^{-}\right)_{\text {weak }}$ & sum & $\left(h^{+}-h^{-}\right)_{\text {all }}$ \\
\hline 0.0 & 0.299 & 0.149 & 1.853 & -0.987 & 1.314 & 1.304 \\
0.4 & 3.433 & 1.163 & 3.517 & -2.489 & 5.624 & 5.558 \\
0.8 & 4.983 & 1.656 & 1.399 & -0.511 & 7.526 & 7.498 \\
1.2 & 4.526 & 1.466 & 0.452 & 0.148 & 6.592 & 6.644 \\
1.6 & 3.642 & 1.016 & 0.159 & 0.392 & 5.209 & 5.170 \\
2.0 & 2.693 & 0.623 & 0.085 & 0.361 & 3.763 & 3.689 \\
2.4 & 1.962 & 0.417 & 0.060 & 0.197 & 2.636 & 2.605 \\
2.8 & 1.525 & 0.464 & 0.039 & 0.124 & 2.153 & 2.159 \\
\hline yield & 9.225 & 2.782 & 3.026 & -1.106 & 13.927 & 13.851 \\
\hline \hline
\end{tabular}

Table 5.3: Contributions to observable $d N / d y$ charge-excess distribution. The difference between the sum and $\left(h^{+}-h^{-}\right)_{\text {all }}$ results from fluctuations in produced charged pions which dominate the charge-excess.

actually measure $\left(h^{+}-h^{-}\right)$all. The values shown were calculated for the modified parameter set assuming full transverse momentum coverage from $0.0<p_{T}<2.0$ $\mathrm{GeV} / c$. For $d N / d y \sim 1$ the statistical errors are $\approx 1 \%$, for $d N / d y \sim 0.1$ the errors are $\approx 4 \%$. The small difference between the sum and $\left(h^{+}-h^{-}\right)$all results from fluctuations in produced charged pions which dominate the charge-excess. Otherwise, the agreement indicates the cancellation in the pion sector which is the basis for the method. The last row in the table is the integrated yield $\int(d N / d y) d y=n_{c h}$ from each column.

Figure 5.19 plots the same four $d N / d y$ distributions. The relatively coarse binning reflects the bin size employed for the measured data. Note that the contribution from hadronic secondaries exceeds that from primary protons in the first

\footnotetext{
${ }^{8}$ Since produced $p$ and $\bar{p}$ should have very nearly the same yields and distributions, the incident protons can be associated with the observable $(p-\bar{p})_{\text {prim }}$. The subscript means primary protons, i.e., protons from secondary processes, for example $\Lambda \rightarrow p \pi^{-}$are excluded. The subscript will be omitted from now on.
} 


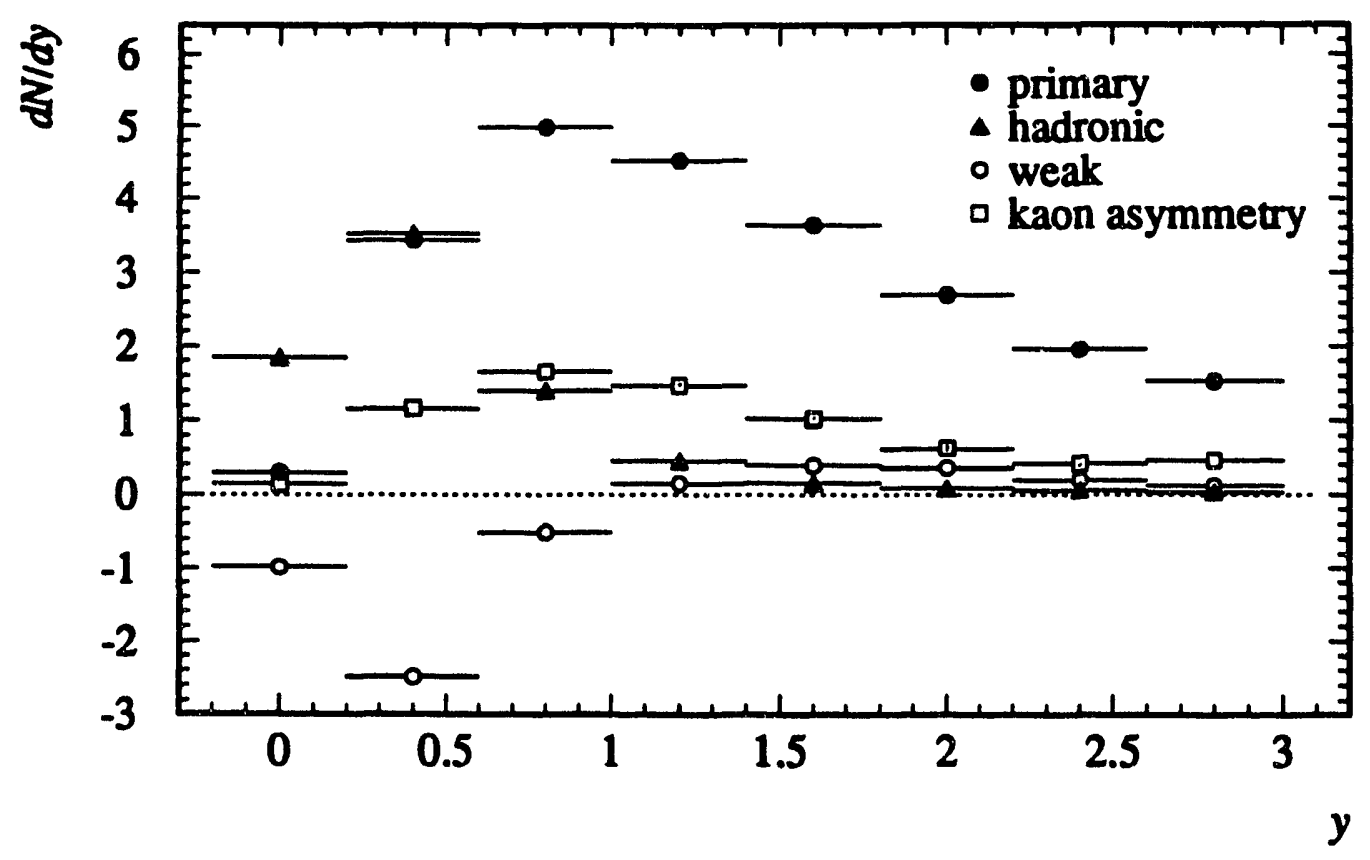

Figure 5.19: Contributions to the the charge-excess distribution $d N / d y$. The charged pions, which cancel, have been omitted. The statistical errors in $d N / d y$ are smaller than the plotting symbols.

two bins. Omitting this correction could lead to a $\sim 20 \%$ overestimate of the number of participant protons. The integrated yield from weak decays is less than zero, by about one charged particle per event. This negative contribution is a kinematic and acceptance effect. Because of its mass, in the laboratory rest frame the proton from $\Lambda$ decay has much higher momentum and $p_{T}$ on average than its companion $\pi^{-}$. Since for the charge-excess distributions the rapidity is calculated assuming the proton mass for all charged particles, the downward rapidity shift for the pions is dramatic. While only $\sim 50 \%$ of the decay protons fall in the acceptance, $\sim 75 \%$ of the pions have $y\left(m_{p}\right)<3.0$. This accounts for the negative yield and the sharp negative values of $d N / d y$ for $y<0.6$.

The kinematic asymmetry of $\Lambda$ decay and the downward rapidity shift of the decay pions leads to a strong $p_{T}$-dependence of $\left(h^{+}-h^{-}\right)_{\text {weak }}$ which changes with rapidity. This is shown in Figure 5.20 which plots the transverse momentum distribution $d N / d p_{T}$ as a function of $p_{T}$ from the weak charge-excess for four different rapidity intervals. Note the different scale of the ordinate for $-0.2<y<0.6$ where the effect is most severe. Although the $p_{T}$ spectra from the $\left(K^{+}-K^{-}\right)$and $\left(h^{+}-h^{-}\right)_{h a d}$ excesses are positive definite for all $y$, the weak decay effect is strong enough to drive the observable distribution $\left(h^{+}-h^{-}\right)$all negative for $p_{T}<200$ $\mathrm{MeV} / c$. It is still strong in the rapidity interval where our proton acceptance is unbiased, $0.6<y<1.4$. This was the most compelling reason for limiting the proton acceptance to $p_{T}>200 \mathrm{MeV} / c$.

The ratios and correction factors to the charge-excess distribution required to 

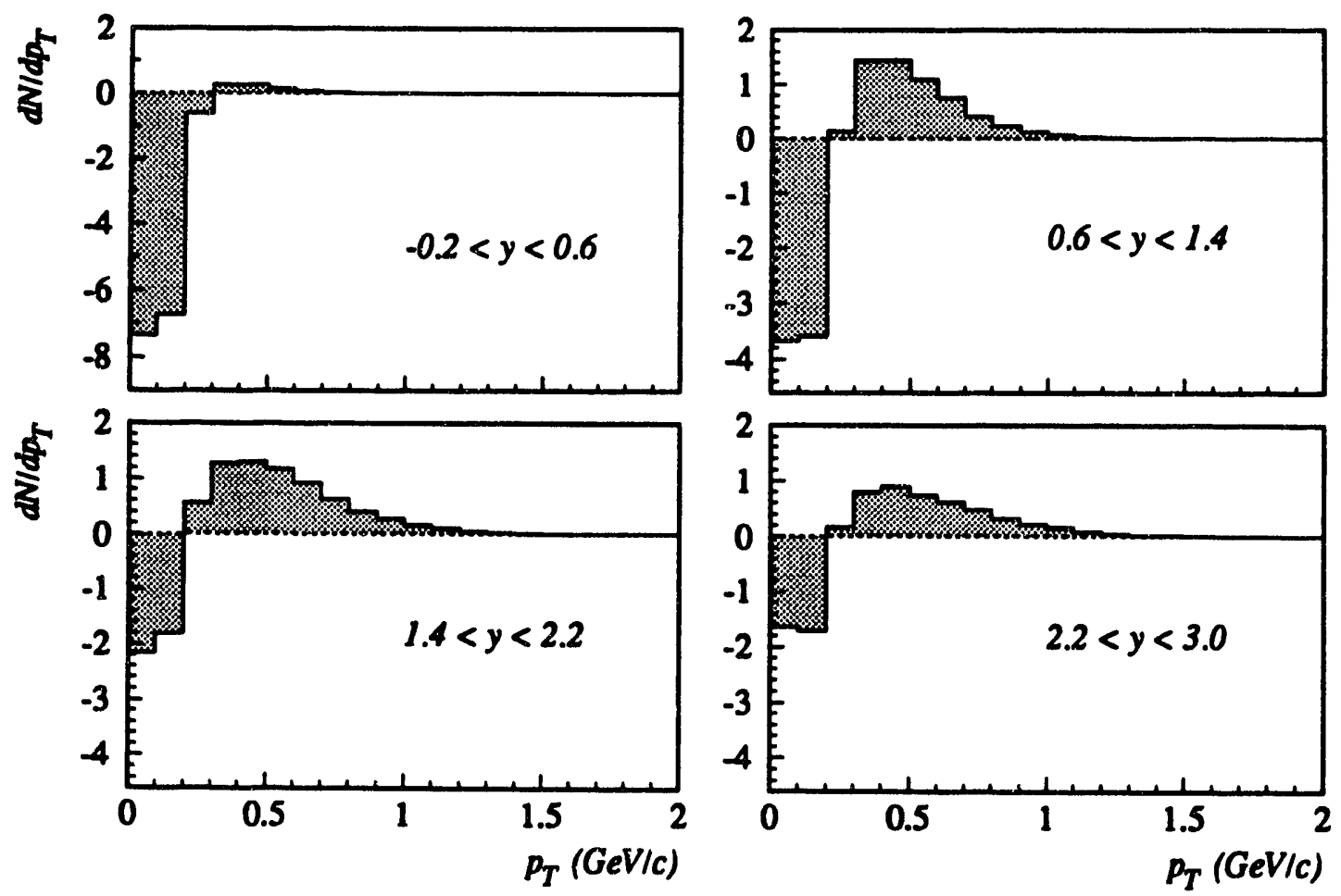

Figure 5.20: Transverse momentum dependence of $d N / d p_{T}$ from $\left(h^{+}-h^{-}\right)_{\text {weak }}$ for different rapidity intervals. The effect is strong enough to drive the observable distribution $\left(h^{+}-h^{-}\right)_{\text {all }}$ negative for $p_{T}<200 \mathrm{MeV} / c$.

unfold the primary proton $d N / d y$ distribution are shown in Figure 5.21. The values differ from those which could be calculated from Table 5.3 because of the inclusion of acceptance effects-the corrections/ratios are derived for $0.6<y<1.4$ with the low- $p_{T}$ cutoff $p_{T}>200 \mathrm{MeV} / c$, elsewhere with the rapidity-dependent low(high)$p_{T}$ cutoffs implied by $y_{p}\left(p_{T}, y_{\pi}=0.6(2.8)\right)$. See Equation 5.5 and Figure 5.5. The severe cutoffs are the reason the statistical errors are so large for $y<0.2$ and $y>2.6$. They are also the reason there are no data points for the default parameter set for $y>2.2$. If the $<p_{T}>\sim 350 \mathrm{MeV} / c$, as it is for FRITIOF with $\operatorname{PAR}(12)=0.35$, our efficiency for detecting protons for $y>2.2$ is just too low. Because of this dependence of the correction on the assumed $\left\langle p_{T}\right\rangle$, the systematic error on the data point at $y=2.4$ is very large in spite of the small statistical error for the modified parameter set. For this reason, and the related difficulty of trying to extrapolate from the tail of the distribution in $p_{T}$, we can only measure the proton $d N / d y$ from $0.2<y<2.2$. The difference in the correction factors for the two different FRITIOF assumptions implies that the systematic error may be as large as $\sim 20 \%$.

Although the shape of the proton $d N / d y$ distribution may be correctly measured the integrated yield will still be low-the number of incident baryons "promoted" to hyperon states must be estimated in order to arrive at the number of participant protons. For processes like

$$
p p \rightarrow p K^{+} \Lambda
$$




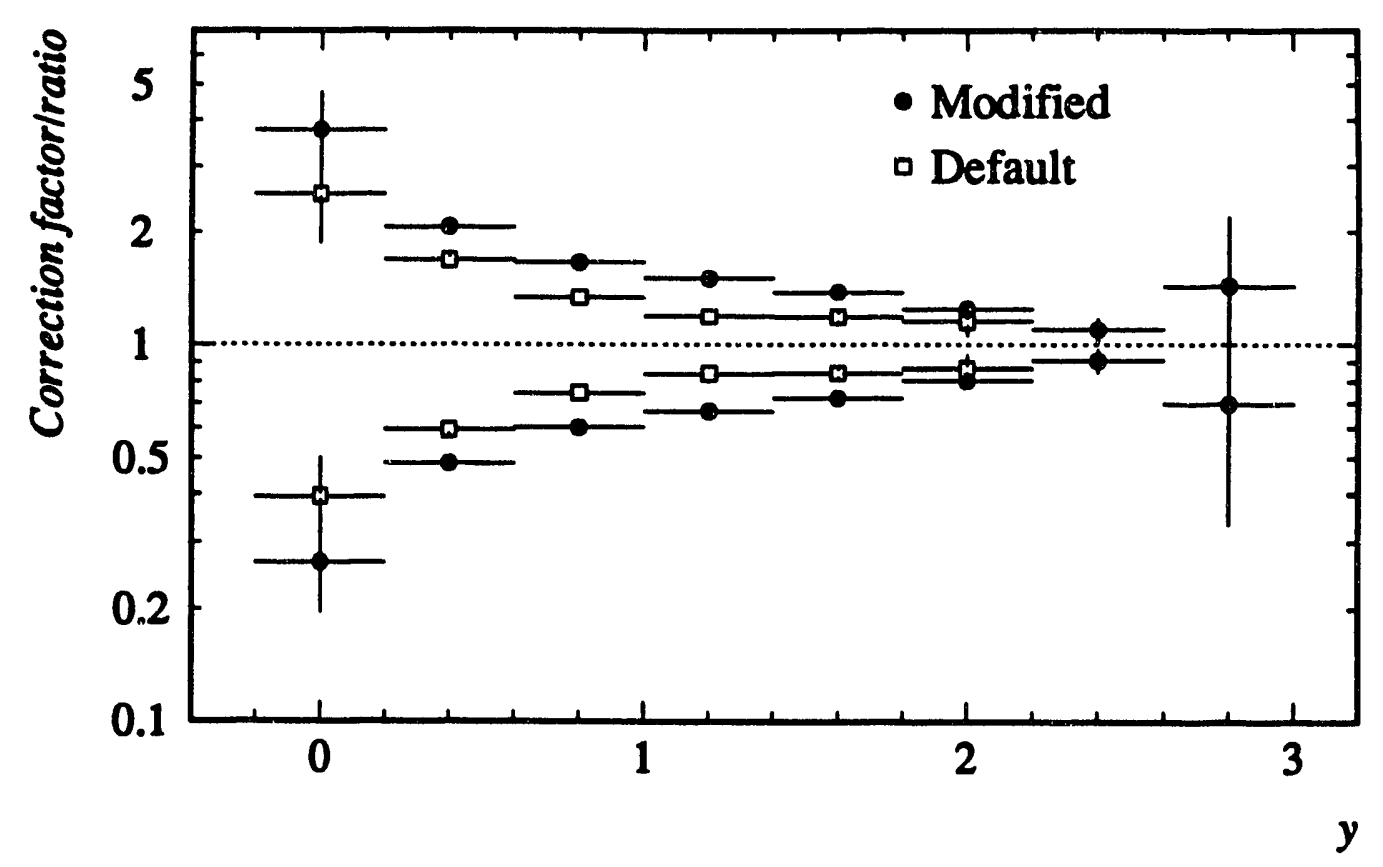

Figure 5.21: Ratios and correction factors for the proton $d N / d y$ distribution. The values are different from the ones implied by Table 5.3 because of the acceptance cutoffs.

$$
\rightarrow p K^{+}\left(p \pi^{-}\right)
$$

our correction compensates for the kinematic and acceptance effects that distort the zero charge-excess from $\Lambda$ decay and the net charge-excess and kinematic effects from $\mathrm{K}^{+}$production. Only one of the four charged particles detected in the final state from the process described in Equation 5.14 can be associated with an incident baryon. One of the incident protons has been lost.

For $p_{T}>200 \mathrm{MeV} / c$ the contributions of weak decays, hadronic secondaries, and the $\mathrm{K}^{+}$excess to the transverse momentum distribution are positive for all $p_{T}$. The ratio of $1 / p_{T} d N / d p_{T}$, the invariant cross section integrated from $0.6<y<1.4$, of observable to primary $\left(h^{+}-h^{-}\right)_{\text {all }} /(p-\bar{p})$ is plotted as a function of $p_{T}$ in Figure 5.22. Also plotted are the bin-by-bin correction factors. The dashed lines are polynomial fits which smooth the correction. The weak chargeexcess drives the ratio down at low $p_{T}$, eventually to a negative value in the first bin. The nearly flat regions from $0.2<p_{T}<1.2 \mathrm{GeV} / c$ are dominated by the charged kaon excess. The average value of the correction factor in this domain is $\sim 0.6$. Secondary hadronic interactions are responsible for the sharp rise for $p_{T}>1.2 \mathrm{GeV} / c$. The dramatic correction for high- $p_{T}$ does not significantly alter the physics we deduce from the proton $p_{T}$ spectrum-the observation of a thermal distribution and high $\left\langle p_{T}\right\rangle$. The temperature $T$ determined from a thermal Boltzmann fit to the data decreases by $\sim 10 \mathrm{MeV}$ after applying the correction. 


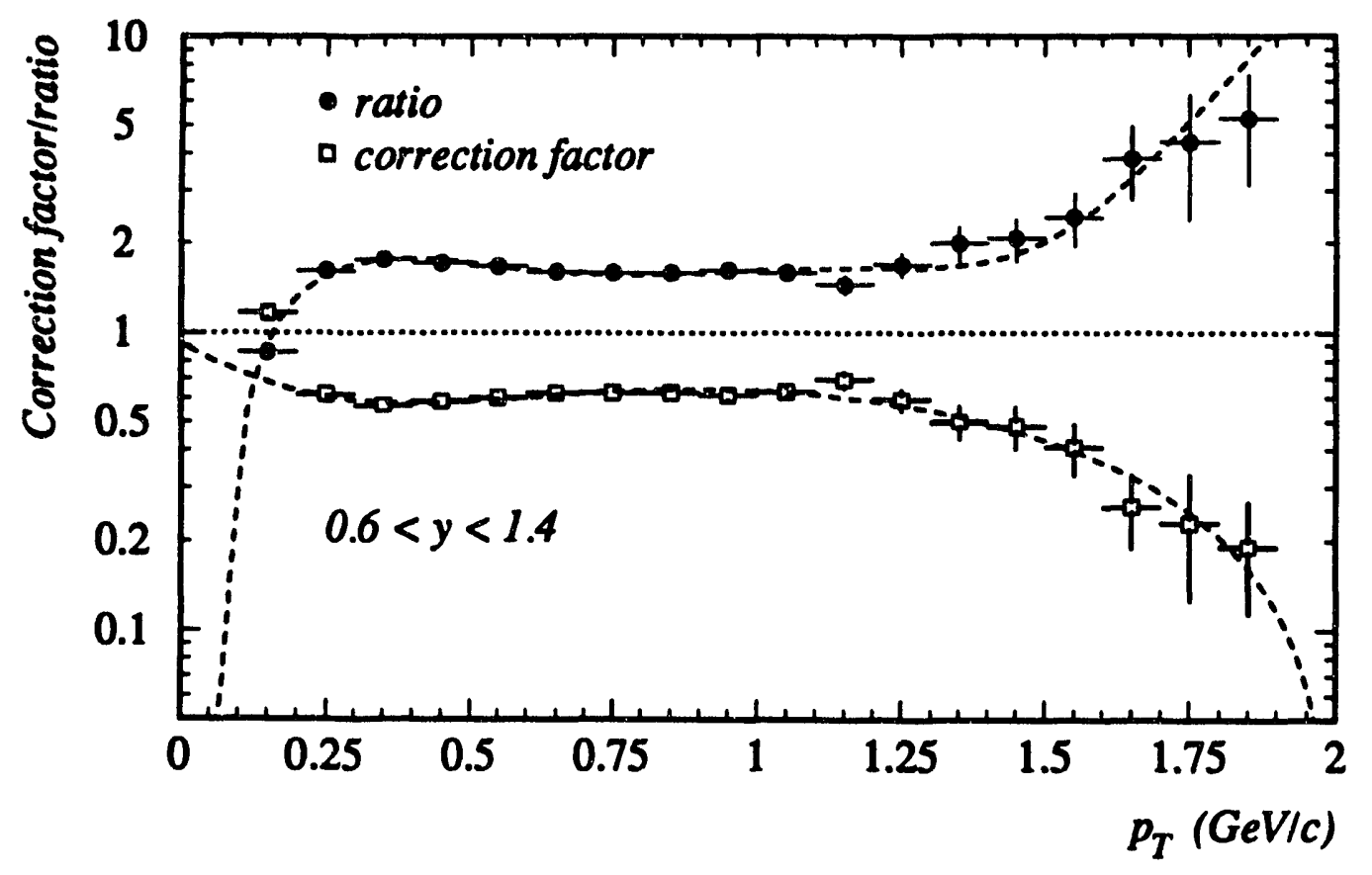

Figure 5.22: Ratios and correction factors for the proton $p_{T}$ distribution in the unbiased rapidity acceptance $0.6<y<1.4$.

\subsection{Extrapolation}

Final rapidity spectra for protons and negative hadrons are obtained by extrapolating the measured distributions to the full transverse momentum range $0.0<p_{T}<2.0 \mathrm{GeV} / c$. Because our pion acceptance is rectangular in $\left(y, p_{T}\right)$ space and reaches down to fairly low $p_{T}$, the extrapolation factors to the negative hadron $d N / d y$ distribution are small and well constrained by the data, $\sim 3.5 \%$ for $0.4<y<2.8$. Because the proton $p_{T}$ acceptance is more limited and strongly dependent on rapidity, the proton $d N / d y$ extrapolation factors are large and change rapidly near the edges of the acceptance. In order to calculate them we assume that the proton $p_{T}$ distribution is independent of rapidity, which may introduce large systematic errors.

Figure 5.23 shows the measured negative hadron transverse momentum.distribution $d N / d p_{T}$ as a function of $p_{T}$ for $p_{T}<0.5 \mathrm{GeV} / c$ in the rapidity interval $1.0<y<1.6$. The data plotted have been corrected for electrons but not for secondary hadrons. The inclusive production of low $p_{T}$ charged pions has been measured at the ISR at $\sqrt{s}=23 \mathrm{GeV}$ [Gue76] and found to be well fitted by an exponential in transverse mass $m_{T}=\sqrt{m^{2}+p_{T}^{2}}$ :

$$
E d^{3} \sigma / d p^{3}=A s^{\alpha} \exp \left(-B m_{T}\right)
$$

For small rapidity intervals this implies

$$
d N / d p_{T}=a p_{T} \exp \left(-m_{T} / T\right)
$$




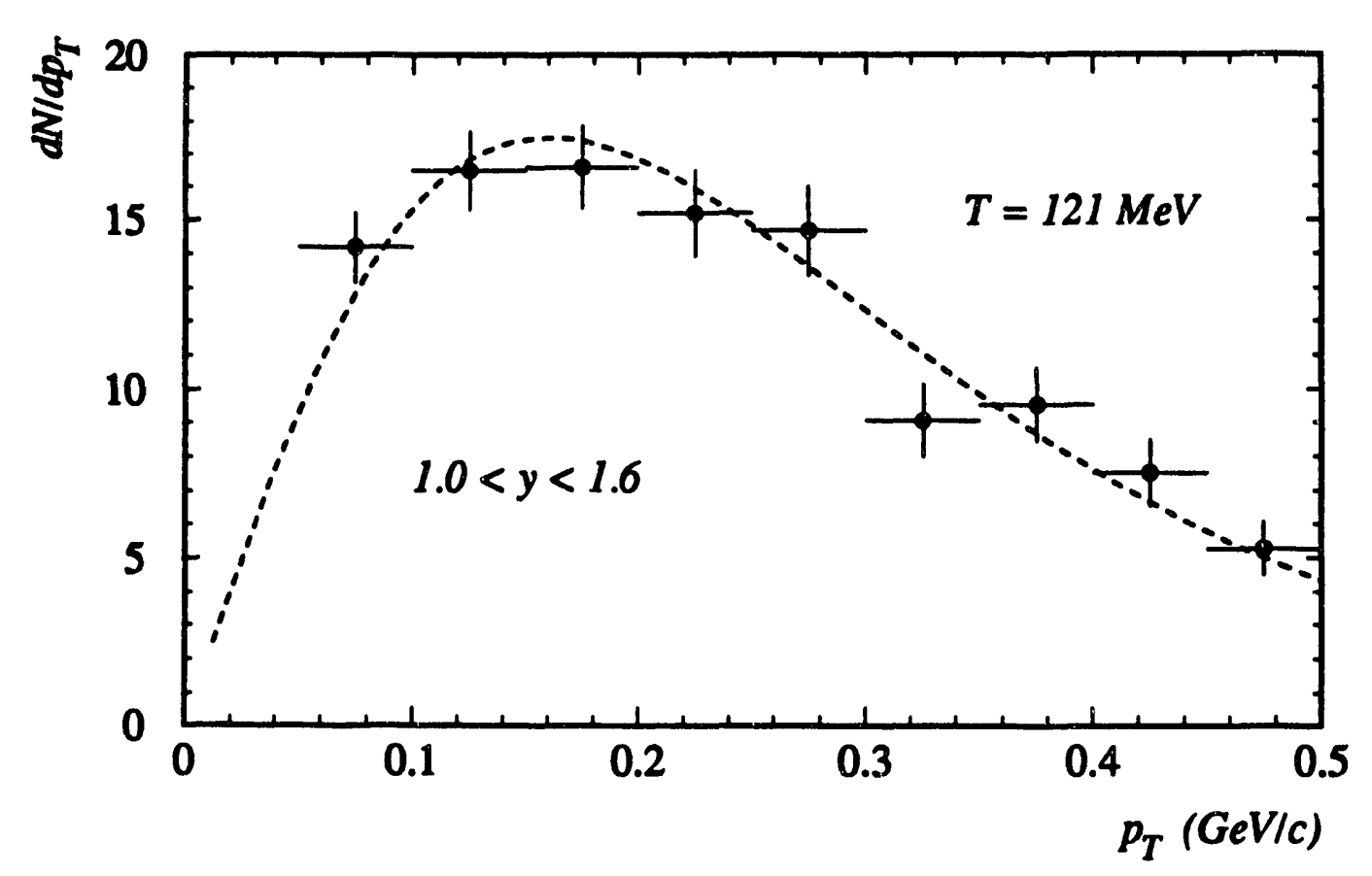

Figure 5.23: Transverse mass $m_{T}$ fit to the negative hadron $p_{T}$ spectrum for extrapolation down to $p_{T}=0$. The data shown have been corrected for electrons but not for secondary hadrons.

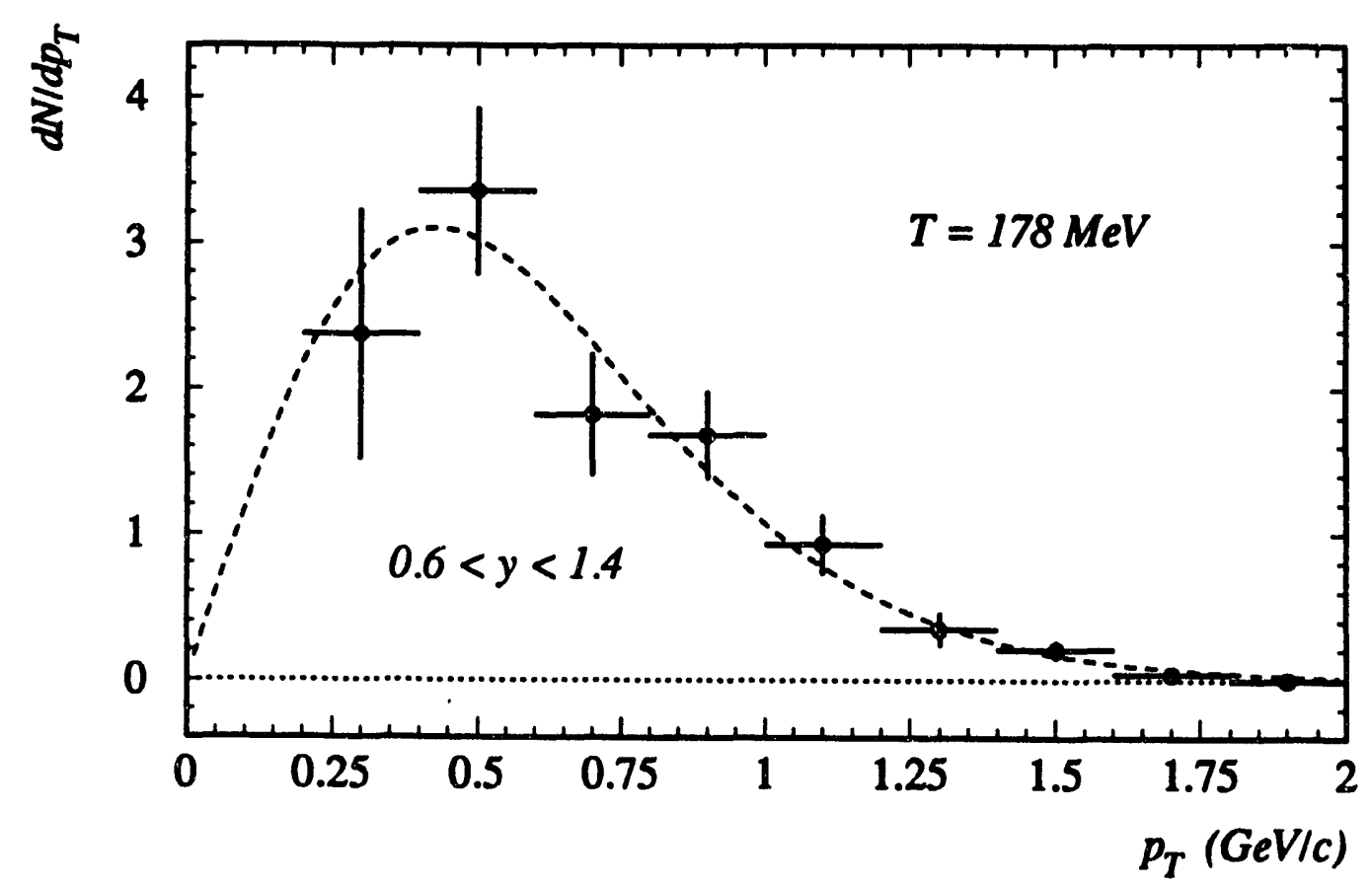

Figure 5.24: Transverse mass $m_{T}$ fit to the proton $p_{T}$ spectrum. The data shown have been fully corrected. 
where the inverse slope parameter $T$ may be loosely associated with temperature. The dashed line in Figure $\mathbf{5 . 2 3}$ is the result of a least-squares fit of Equation $\mathbf{5 . 1 6}$ to the data for $T=121 \mathrm{MeV}$. From the fit, a value was assigned to the low pr bin centered at $p_{T}=25 \mathrm{MeV} / c$, leading to an extrapolation factor of $3.5 \%$. The procedure was repeated in four adjacent rapidity intervals. Since the measured $\left\langle p_{T}\right\rangle$ increases with rapidity, the extrapolation factors decrease. The dependence on rapidity is approximately linear so the bin-by-bin factors $f(y)$ were calculated from a linear fit

$$
f(y)=1.0386-0.00335 y \text {. }
$$

Because the change in $f(y)$ with rapidity is weak, the shape of the negative hadron $d N / d y$ distribution is fairly insensitive to the extrapolation.

Because the rapidity dependence of both the low- and high- $p_{T}$ acceptance cutoffs for protons is severe, we extrapolate the proton $d N / d y$ distribution by a Monte Carlo technique based on a fit to the fully corrected transverse momentum spectrum in the unbiased acceptance interval $0.6<y<1.4$. We again employ Equation 5.16 which is a frequently used approximation [Abb90], [Bam89] to the correct expression for an isotropic, thermal Boltzmann distribution at temperature $T_{B}$,

$$
d N / d p_{T}=a p_{T} m_{T} \exp \left(-m_{T} / T_{B}\right)
$$

The fully corrected proton $d N / d p_{T}$ distribution, along with the result of the $m_{T}$ fit for $T=178 \mathrm{MeV}$, is shown in Figure 5.24. Although the temperature parameter $T$ is typically $\sim 25 \mathrm{MeV}$ higher than $T_{B}$, as parametrizations of the data the $m_{T}$ and Boltzmann fits are indistinguishable, especially for $m \gg T, T_{B}$. The differences between the correct and exact expressions for the case $T=T_{B}$ are carefully examined in Ref. Hag83.

For each rapidity bin we generate a flat rapidity distribution $d N / d y_{p}=$ constant where $y_{p}$ means rapidity calculated with the proton mass. We obtain points in $\left(y_{p}, p_{T}\right)$ space by randomly sampling from the $m_{T}$ distribution with $T=178 \mathrm{MeV}$, map the values to $y_{\pi}$, then apply the acceptance cuts $0.6<y_{\pi}<2.8$, and $p_{T}>200$ $\mathrm{MeV} / c$. The calculation is illustrated for the rapidity bins $y_{p}=[0.2,0.6],[1.8,2.2]$ in Figure 5.25. The hatched areas represent the disallowed regions outside of the acceptance. The bin-by-bin extrapolation factors are calculated from the ratio of the number of points generated in $\left(y_{p}, p_{T}\right)$ space to the number of points accepted in $\left(y_{\pi}, p_{T}\right)$ space.

The results of the Monte Carlo extrapolation are shown in the second column of Table 5.4. Also shown for comparison are factors calculated from FRITIOF in two ways. The column labelled "FRITIOF" is the exact result obtained from the ratio of the proton $d N / d y$ distribution with and without the acceptance cuts. It makes no assumption about the shape of the $d N / d y$ distribution within a bin, nor about the $\left\langle p_{T}>\right.$, nor about the form or rapidity dependence of the $p_{T}$ distribution. The entries in the column denoted "FRITIOF $M C$ " are calculated in the same way as the values determined from the measured data except that the $d N / d p_{T}$ distributions employed are extracted from FRITIOF and are different for each bin. The close agreement between the two FRITIOF calculations indicates 

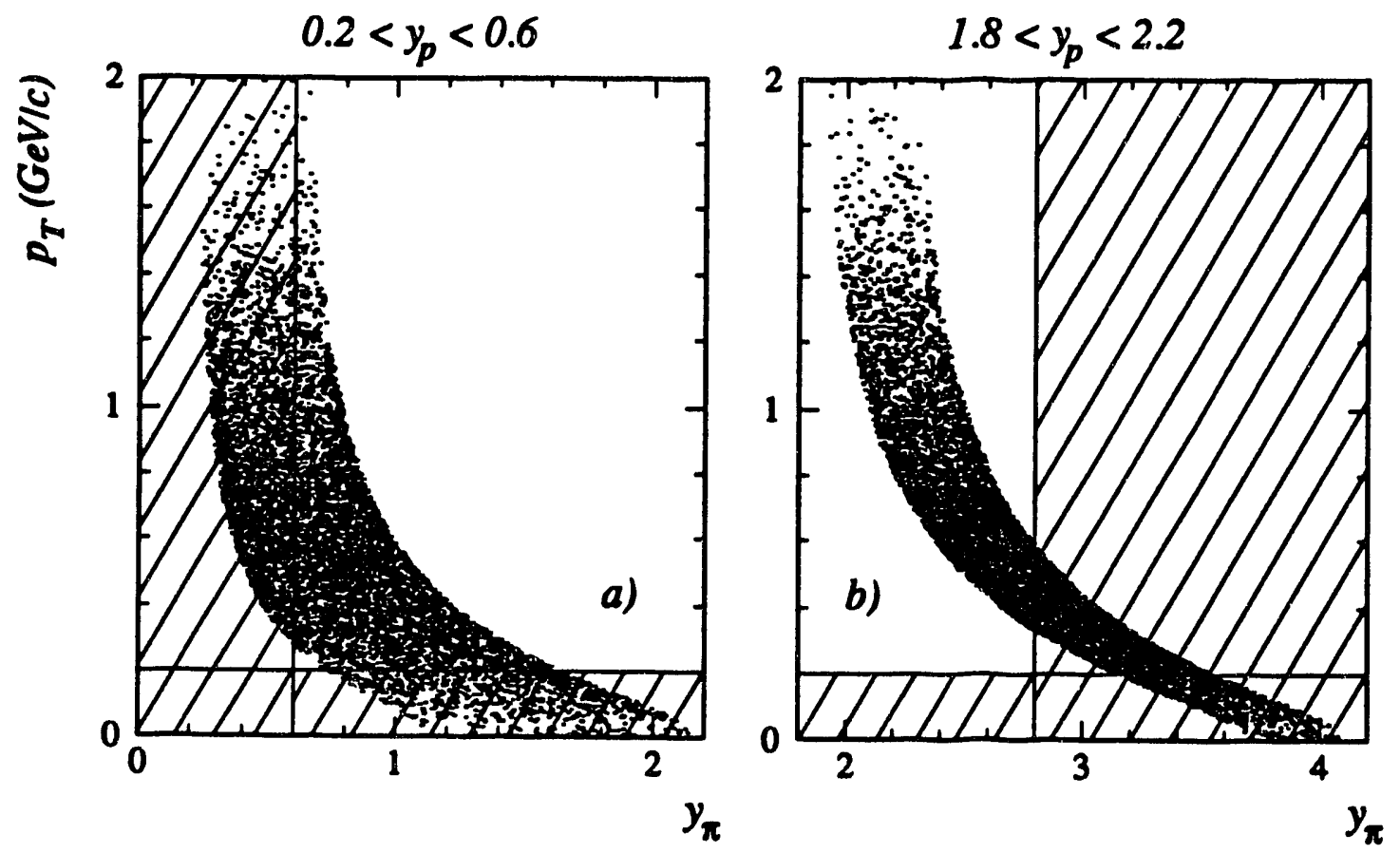

Figure 5.25: Monte Carlo extrapolation of proton $d N / d y$ distribution. Panel a(b) shows the result of mapping $\left(y_{p}, p_{T}\right)$ from the proton rapidity interval $\Delta y_{p}=$ $[0.2,0.6]([1.8,2.2])$ to rapidity calculated with the pion mass $y_{\pi}$. The hatched regions indicate the acceptance cutoffs.

\begin{tabular}{||c|c|c|c|c||}
\hline \hline bin & $\Delta y$ & Monte Carlo & FRITIOF & FRITIOF $_{M C}$ \\
\hline 1 & {$[0.2,0.6]$} & 1.674 & 1.401 & 1.537 \\
2 & {$[0.6,1.0]$} & 1.106 & 1.181 & 1.180 \\
3 & {$[1.0,1.4]$} & 1.106 & 1.166 & 1.163 \\
4 & {$[1.4,1.8]$} & 1.176 & 1.226 & 1.236 \\
5 & {$[1.8,2.2]$} & 1.626 & 1.792 & 1.770 \\
6 & {$[2.2,2.6]$} & 4.423 & 4.962 & 4.980 \\
\hline
\end{tabular}

Table 5.4: Monte Carlo extrapolation factors for the proton $d N / d y$ distribution. 
that the Monte Carlo procedure, in particular the use of a flat $d N / d y$ distribution to populate the proton phase space, yields the correct result except for the first bin. For the rapidity interval $\Delta y=[0.2,0.6]$ the FRITIOF $d N / d y$ distribution is changing too rapidly which leads to an overestimate of the extrapolation factor. Note that the FRITIOF $M C$ value is larger than the data-based Monte Carlo one except for the first bin where it is smaller. This results from the assumption that the full transverse momentum phase space out to $p_{T}=2.0 \mathrm{GeV} / c$ is accessible near target rapidity. The proton phase space in FRITIOF does not fully "open" until $y \sim 1.0$. These comparisons suggest that the systematic error in bin 1 may be as large as $\sim 20 \%$. In bins $2-5$ we estimate the systematic error to be less than $\sim 10 \%$ from Table 5.4 and from the changes in the Monte Carlo extrapolation factors which result from varying the $\left\langle p_{T}\right\rangle$ with rapidity while assuming the shape remains the same. The large extrapolation factor for $\Delta y=[2.2,2.6]$ makes a data point there unreliable-it would have to be determined from a low statistics measurement of the tail of the $p_{T}$ distribution. This limits the range of our proton $d N / d y$ measurement to $0.2<y<2.2$.

\subsection{Summary of Corrections and Data Sample}

Of the 218 events measured, 216 were retained for analysis as valid target interactions. After matching and reconstruction this data set contained 17,923 charged particles of which 14,475 were two-view reconstructions and 3,448 were three-view reconstructions. $9,571(8,352)$ were positively(negatively) charged. The final data sample employed for the construction of charged particle spectra was selected from the matched and reconstructed data by applying four selection cuts:

1. Three-view acceptance and $w_{i}(p, \theta, q)<8.0$. See Equation 5.3.

2. $\chi_{v t x}^{2}<\chi_{\max }^{2}=71.25$ determined from a fit to the exponential core of the measured $\chi_{v t x}^{2}$ distribution and the requirement of a tail probability $P\left(\chi_{v t x}^{2}\right)>.001$.

3. Acceptance limits for the negative hadron $d N / d y$ and $p_{T}$ spectra, $p_{T}>50$ $\mathrm{MeV} / c$ and $0.4<y_{\pi}<2.8$.

4. Acceptance limits for the proton $d N / d y$ and $p_{T}$ spectra, $p_{T}>200 \mathrm{MeV} / \mathrm{c}$ and $0.6<y_{\pi}<2.8$.

The influence of the four cuts on the number of charged particles which remain in the data sample is summarized in Table 5.5.

For the negative hadron $p_{T}$ and $d N / d y$ spectra each charged particle satisfying the selection cuts is assigned histogram weights $W_{p_{T}}$ and $W_{y}$, respectively,

$$
\begin{aligned}
W_{P T} & =A w_{i} c_{i} / p_{T} \\
W_{y} & =A w_{i} c_{i},
\end{aligned}
$$




\begin{tabular}{||l|c|c|}
\hline \hline cut & ch $>0$ & ch $<0$ \\
\hline matched & 9571 & 8352 \\
1 & 8725 & 7590 \\
$1+2$ & 7813 & 6803 \\
$1+2+3$ & & 5260 \\
$1+2+3+4$ & 4108 & 2962 \\
\hline \hline
\end{tabular}

Table 5.5: Influence of selection cuts on data sample.

where $w_{i}$ is the geometrical weight and $c_{i}=c_{i}\left(y, p_{T}\right)$ is the electron correction. $A$ is a normalization factor so that the integral of either spectrum yields the mean number of charged particles. For the proton spectra the electron correction terms are omitted. From Equation 5.19 the statistical error on histogram bin $j$ of the $d N / d y$ spectra is

$$
\sigma_{j}^{2}=A^{2} \sum_{i=1}^{N_{j}}\left(w_{i} c_{i}\right)^{2}+c_{i}^{2} \sigma_{w_{i}}^{2}+w_{i}^{2} \sigma_{c_{i}}^{2}
$$

where $N_{j}$ is the number of particles in the bin, with an analogous expression for the $p_{T}$ spectra. The statistical errors in the geometrical weights $\sigma_{w_{i}}$ and in the electron correction $\sigma_{c_{i}}$ were estimated in Sections 5.2 and 5.3. The error is dominated by the first term.

Final transverse momentum spectra are obtained from the efficiency-corrected measured distributions by multiplying the unfolded histograms by the bin-by-bin correction factors determined from our analysis of non-vertex particles. The final negative hadron $d N / d y$ distribution is obtained by first extrapolating the efficiencycorrected spectrum, then applying the secondary particle correction. To obtain the final proton $d N / d y$ distribution, we first correct the measured spectrum for secondary charged particles, then apply the Monte Carlo extrapolation. The statistical errors of the corrections are added in quadrature to the statistical errors calculated from Equation 5.20. The systematic errors have been estimated as they have been discussed. 


\section{Chapter 6}

\section{Charged Particle Spectra}

\subsection{Rapidity Distributions}

In this section we present the fully corrected rapidity distributions for negative hadrons and protons. Both the negative hadron and proton data can be accommodated in a simple picture based on the assumptions of limited nuclear stopping, evolution to a state of thermal equilibrium, followed by collective, primarily longitudinal, expansion.

The rapidity density of negatively-charged hadrons $d N / d y$, normalized to the mean negative multiplicity and calculated assuming the pion mass, is shown in Figure 6.1 and compared to the distribution obtained from FRITIOF. Only the backward hemisphere in the center-of-mass $y<3.0$ is displayed because of the limits of our acceptance. According to FRITIOF, approximately $10 \%$ of the charged hadrons are kaons. The FRITIOF histogram was compiled for the modified parameter set of Table 5.1 with events selected by impact parameter $b$ by $b d b$ weighting for $b<b_{\max }=2.5 \mathrm{fm}$. As noted in Chapter 5 , this event selection leads to a good overall normalization of the Monte Carlo to the data.

From our best fit below, the distribution peaks at midrapidity with $d N / d y=$ $19.94 \pm 0.71$ charged particles per unit of rapidity and an integrated yield for $0.0<y<3.0$ of $35.31 \pm 0.59$ charged particles compared to $38.27 \pm .13$ for the simulation. The errors, which are statistical only and depend on extrapolating the fit, have been calculated by a Monte Carlo method ${ }^{1}$ which assumes the data points and their error bars represent the mean values and standard deviations of Gaussian distributions. By generating a large ensemble of spectra from the data and fitting each member, one obtains distributions for the fit parameters, extrapolated points and integrated yield whose values and widths correspond to the estimates and errors stated above. The FRITIOF distribution is somewhat broader than the data. The widths of the measured data and the FRITIOF spectrum, assuming symmetry about the center-of-mass $y_{\mathrm{cm}}=3.0$, are $\Delta y_{\mathrm{FWHM}}=3.5$ and $\Delta y_{\mathrm{FWHM}}=4.0$, respectively. In fully inclusive, minimum bias $p p$ data at $200 \mathrm{GeV} / \mathrm{c}$

\footnotetext{
${ }^{1}$ We thank E. Friedlander for suggesting this approach to determining the errors on numbers which depend on extrapolating fits to measured distributions.
} 


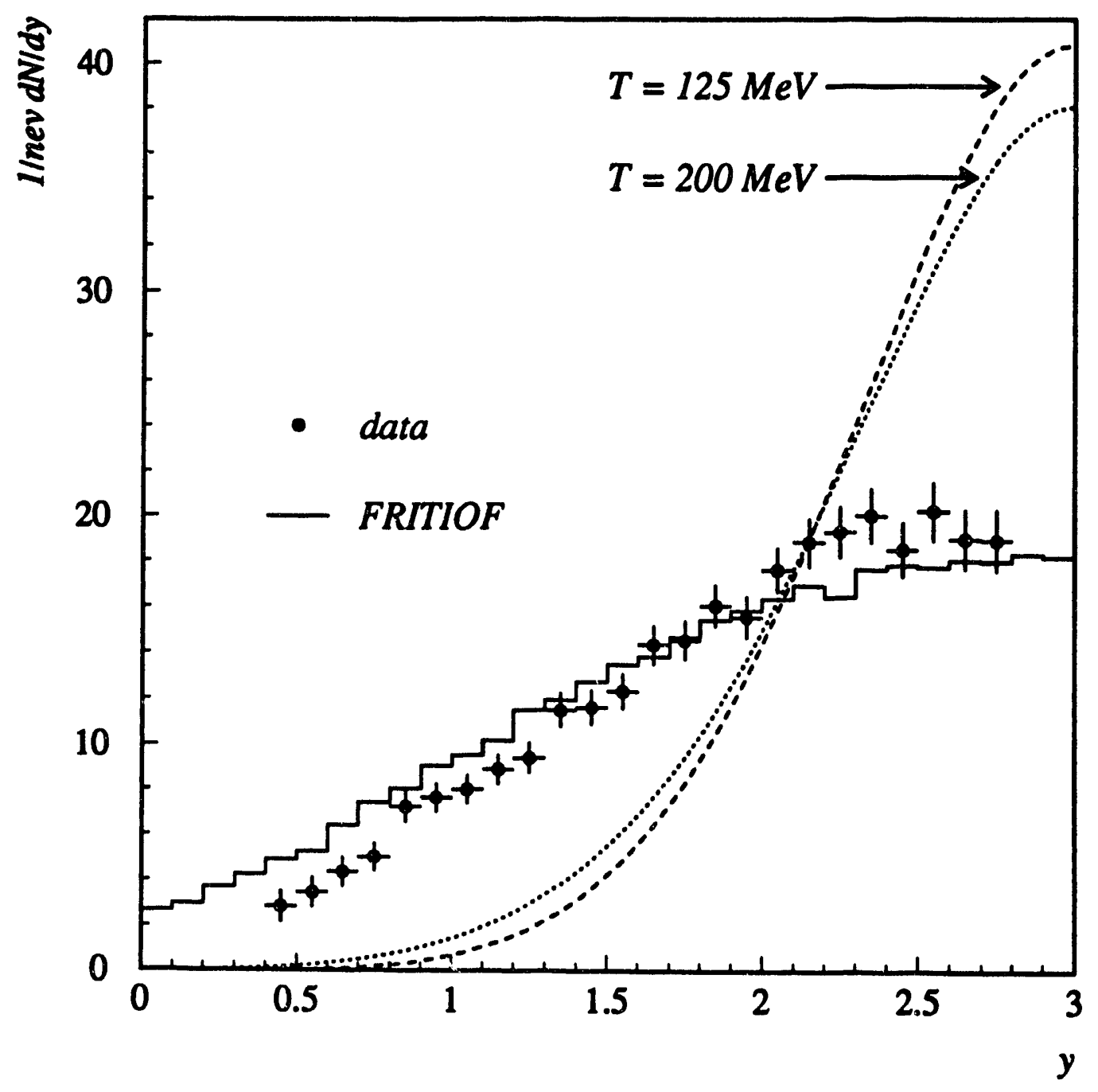

Figure 6.1: Fully corrected negative hadron $d N / d y$ distribution. The data are compared to the predictions of FRITIOF and the isotropic thermal model at $T=125$ and $T=200 \mathrm{MeV}$. Only statistical errors are shown. 
[DeM82a] the rapidity distribution of negative hadrons was symmetric about the rapidity of the nucleon-nucleon center-of-mass $y_{N N}$ with a width about equal to the ${ }^{32} \mathrm{~S}+{ }^{32} \mathrm{~S}$ data $\Delta y_{\mathrm{FWHM}} \approx 3.5$. For similarly triggered $p \mathrm{Xe}$ data at the same energy the negative hadron $d N / d y$ distribution was skewed about a maximum value $y_{\max }<y_{N N}$ and narrower than that for $p p$ [DeM82a]. This is in qualitative agreement with the $\mathrm{NA35}$ result for $200 \mathrm{GeV} /$ nucleon ${ }^{16} \mathrm{O}+{ }^{197} \mathrm{Au}$ data [Str88] where forward of the effective center-of-mass the distribution was also broader and relatively depleted and where the width was found to be $\Delta y_{\mathrm{FWHM}}=3.1$. These observations suggest that symmetric nucleus-nucleus collisions of relatively light ions resemble superpositions of $p p$ interactions while asymmetric collisions of light projectiles with heavy targets are more like superpositions of $p A$ interactions and are significantly influenced by secondary rescattering effects in spectator matter.

The data are consistent with either a peak at midrapidity, a central plateau of about \pm 1 unit of rapidity about $y_{\mathrm{cm}}$, or a depression at midrapidity. A depression of the negative hadron $d N / d y$ distribution was observed [Bel85] in semi-inclusive spectra for $p p$ and $\alpha \alpha$ collisions at $\sqrt{s} \simeq 30$ for events with the lowest integrated negative multiplicity $n_{-}<2$ charged particles. The semi-inclusive data may be a more natural comparison set than the fully inclusive distributions because central $A A$ collisions may still be superpositions of peripheral $p p$ interactions. We will attempt to quantify this hypothesis later by normalizing the produced particles to the number of participant nucleons, which can be inferred from the proton $d N / d y$ spectrum.

The ${ }^{32} \mathrm{~S}+{ }^{32} \mathrm{~S}$ rapidity distribution is much broader than that which would be observed for isotropic emission from a single thermal fireball at temperature $T$ at rest in the center-of-mass. The dashed lines in Figure 6.1 are the predictions for a single source Boltzmann distribution

$$
\begin{gathered}
\frac{d N}{d y} \propto\left(0.5+1 / z+1 / z^{2}\right) \exp (-z), \\
z=m_{0} \cosh \left(y-y_{0}\right) / T_{B}
\end{gathered}
$$

for $m_{0}=m_{\pi}, y_{0}=y_{c m}=3.0$ and $T_{B}=125$ and $200 \mathrm{MeV}$. The Boltzmann curves are normalized to have the same integrated value as the measured data. From lattice QCD calculations [Cle86] the canonical estimate for the critical temperature $T_{c}$ for a first-order phase transition from hadronic matter to the quark-gluon plasma is $T_{c} \sim 200 \mathrm{MeV}$. The E802 collaboration has obtained a satisfactory fit with Equation 6.1 to their mass-identified pion data from ${ }^{28} \mathrm{Si}+{ }^{197} \mathrm{Au}$ central collisions at $14.6 \mathrm{~A} \mathrm{GeV} / c$ [Abb90] by setting $T_{B}=125 \mathrm{MeV}$, the temperature they deduced from their transverse momentum distributions. However the E802 data is heavily extrapolated because of limited and strongly rapidity-dependent $p_{T}$ acceptance and covers less than 1.3 units of rapidity. Chapman and Gyulassy [Cha91] have pointed out that the published E802 measurements may have large normalization errcrs and may not conserve longitudinal momentum. Emulsion data from ${ }^{16} \mathrm{O}$ induced reactions at $14.6 \mathrm{~A} \mathrm{GeV} / \mathrm{c}$ have been fitted with a Gaussian approximation to an 
isotropic distribution in pseudorapidity $\eta[\mathrm{Ger} 89]$,

$$
\frac{d N}{d \eta} \propto 1 / \cosh \left(\eta-\eta_{0}\right)^{2} \approx \frac{1}{\sqrt{2 \pi \delta^{2}}} \exp \left(-\left[\eta-\eta_{0}\right]^{2} / 2 \delta^{2}\right),
$$

with a fixed width $\delta \approx 0.88$ but their best fit is still poor and much narrower than their data. The Boltzmann form for $d N / d y$ is insensitive to $T_{B}$ for $T_{B} / m \gtrsim 1$ with an approximately constant width $\Delta y_{F W H M} \approx 1.8$. Our negative hadron data cannot be fit to a single isotropic thermal distribution at any temperature. The naive expectation that the produced particle $d N / d y$ distribution will get more sharply peaked, i.e, more thermal, in $A A$ collisions has not been substantiated by the data. The semi-inclusive $d N / d y$ spectra from $\alpha \alpha$ collisions at the ISR were narrower for events with higher integrated multiplicity $n_{-}$[Bel85]. Increasing $n_{-}$ should be correlated with smaller impact parameters or larger numbers of nucleonnucleon interactions. However, the same trend was observed in the semi-inclusive ISR $p p$ data suggesting that the narrowing is a hard $p p$ effect rather than the intimation of an approach to thermalization for increasing numbers of nucleonnucleon collisions.

One way to preserve a thermodynamic interpretation, in spite of the failure of an isotropic fireball to reproduce the observed negative hadron $d N / d y$ spectrum, is to incorporate collective final state effects which account for the broad distribution. Stachel and Braun-Munzinger [Sta89a], [Sta89b] have argued that experimental results from the Brookhaven AGS and CERN SPS heavy ion programs are consistent with the Landau hydrodynamic model [Lan65] and indicate that the invariant cross sections factorize into longitudinal and transverse components. By assuming a form for the invariant cross section which explicitly includes Landau's Gaussian expression in rapidity ${ }^{2}$

$$
E \frac{d^{3} \sigma}{d^{3} p}=f\left(p_{T}\right) g(y) \propto \exp \left(-\left[y-y_{0}\right]^{2} / 2 \sigma^{2}\right) \sqrt{m_{T}} \exp \left(-m_{T} / T\right)
$$

they were able to account for the measured transverse energy and charged particle multiplicity distributions at $14.6 \mathrm{~A} \mathrm{GeV} / c$ and $200 \mathrm{~A} \mathrm{GeV} / c$. We will call Equation 6.3 the Landau fireball. The ansatz for $f\left(p_{T}\right)$ is an approximation, for the case $m_{T} / T$ large, to the exact result obtained by integrating a thermal isotropic distribution over the longitudinal degree of freedom. It is claimed to be correct even if collective motions are present provided they are predominantly longitudinal, as they are in the Landau hydrodynamic model, and if $T$ depends only weakly on longitudinal velocity [Hag83].

von Gersdorff et al. [Ger89] analyzed pseudorapidity distributions $d N / d \eta$ from nucleus-nucleus collisions in $\mathrm{AgBr}$ emulsions at $14.6 \mathrm{~A}, 60 \mathrm{~A}$, and $200 \mathrm{~A} \mathrm{GeV} / \mathrm{c}$ and were able to fit all their data to the Landau form

$$
\frac{d N}{d \eta}=\frac{N}{\sqrt{2 \pi \sigma^{2}}} \exp \left(-\left[\eta-\eta_{0}\right]^{2} / 2 \sigma^{2}\right) .
$$

\footnotetext{
${ }^{2}$ The approximate expression appearing in Landau's original paper is for the pseudorapidity density $d N / d \eta$ as in Equation 6.4. The substitution $\eta \rightarrow y$ is justified in Ref. Car72.
} 
Moreover their fitted values for the widths were in good agreement with the explicit, energy-dependent predictions of an extension [Shu72] of Landau's model

$$
\sigma_{L}^{2}=\frac{8}{3} \frac{c_{0}^{2}}{1-c_{0}^{4}} \ln \gamma_{\mathrm{cm}}
$$

In Equation 6.5, $c_{0}$ is the speed of sound in nuclear matter. For the case of an equation of state of non-interacting massless bosons $p=c_{0}^{2} \epsilon=1 / 3 \epsilon$ where $p$ is the pressure and $\epsilon$ the energy density, this expression for $\sigma_{L}^{2}$ reduces to Landau's original prediction

$$
\sigma_{L}^{2}=\frac{1}{2} \ln \left(s / 4 m_{p}^{2}\right)
$$

The Gaussian form for the rapidity distribution has also been successfully applied by Carruthers and Duong-Van [Car73] to pp collision data at $205 \mathrm{GeV} / c$.

The result of a Landau fireball fit to our data is shown as a dashed line in Figure 6.2. Because we cannot measure past midrapidity, we employ the assumption

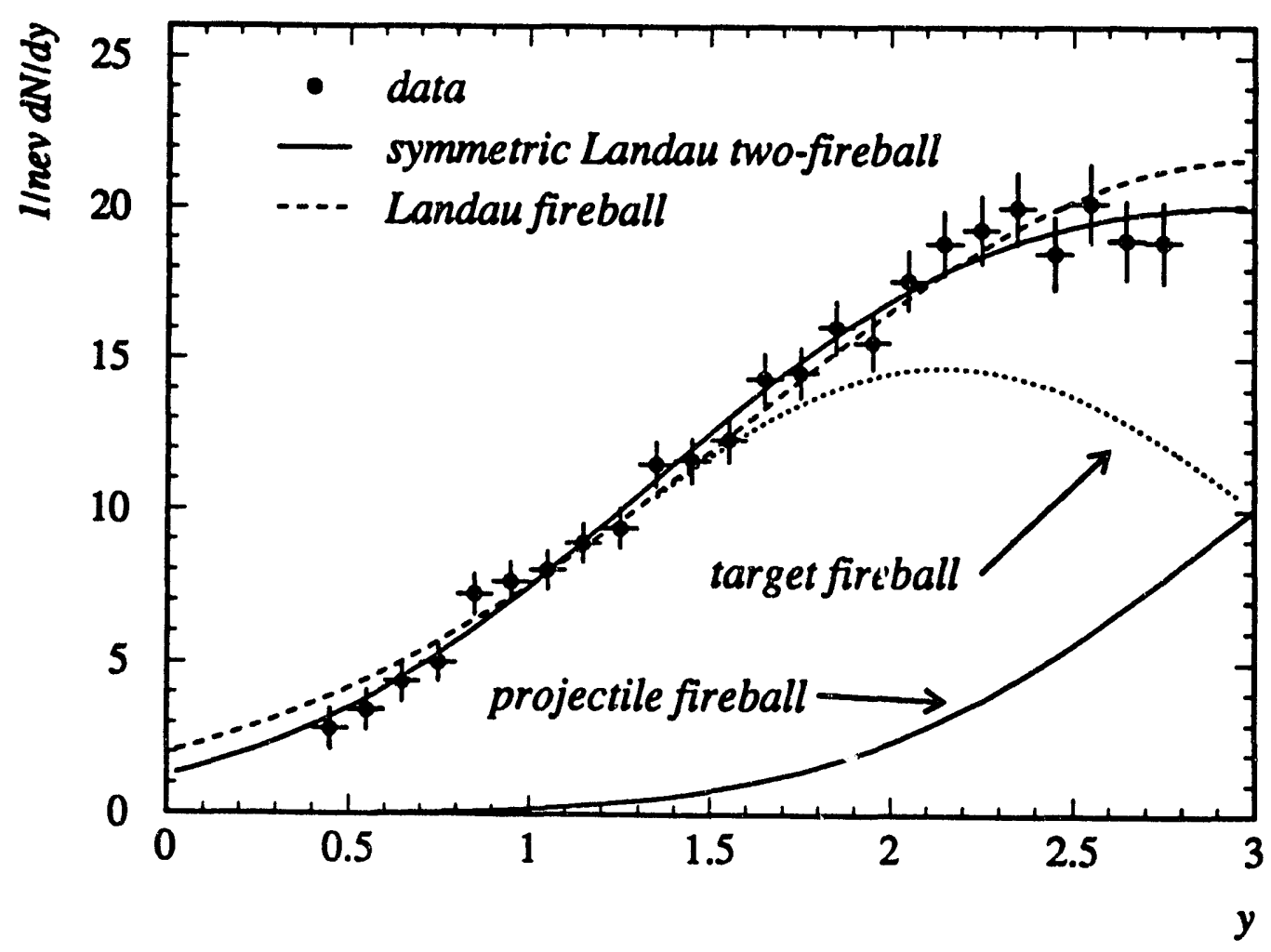

Figure 6.2: Landau fireball fits to the negative hadron $d N / d y$ distribution. The solid line is the result of the symmetric two-fireball fit, the dashed line of the single fireball with the constraint $y_{0}=3.0$.

of symmetry about midrapidity and constrain the distribution to peak at $y_{0}=3.0$. For collisions between nuclei with equal mass numbers, the nucleus-nucleus centerof-mass $y_{\mathrm{cm}}$ equals $y_{N N}$ for all impact parameters. The single Landau fireball fit 
appears to overshoot the data at the peak and in the tail of the $d N / d y$ distribution. von Gersdorff et al. observed a similar deviation at target pseudorapidities. From the fit to the the Gaussian expression for $g(y)$ in Equation 6.3, we find $\sigma=1.376 \pm .025$ in agreement with the result in Ref. Ger89 for their highest multiplicity data set at the same energy but somewhat smaller than the Landau prediction $\sigma_{L}(s)=1.53$.

Also shown in Figure 6.2 is a fit to the two-fireball expression

$$
g(y)=A\left\{\exp \left(-\left[y+y_{0}-y_{c m}\right]^{2} / 2 \sigma^{2}\right)+\exp \left(-\left[y-y_{0}-y_{c m}\right]^{2} / 2 \sigma^{2}\right)\right\},
$$

a natural extension of the Landau fireball model which incorporates limited nuclear stopping and retains the symmetry requirement for equal mass $A A$ collisions that $g\left(y^{\prime}\right)=g\left(-y^{\prime}\right)$ where $y^{\prime}$ is the rapidity measured with respect to the centerof-mass. In the picture we have in mind, the participant nucleons are excited and slowed down but not complelety stopped. In Equation 6.7, $y_{0}>0$ is the distance of projectile and target fireballs from $y_{\mathrm{cm}}$ and $\sigma$ the single width demanded by symmetry. Klar and Hüfner employed the same expression [Kla85] to fit the negative particle data from $p p$ collisions at $200 \mathrm{GeV} / c$ [DeM82a]. They justified the decomposition into two components by the observation that the ratio $R(y)$ of the rapidity distributions of negatively charged particles in $p A$ and $p p$ data

$$
R(y)=\left(\frac{d N}{d y}\right)_{p A} /\left(\frac{d N}{d y}\right)_{p p}
$$

only increased with target mass $A$ for $y \lesssim y_{c m}$ which led them to postulate a targetlike and projectilelike component. They also invoked the theoretical argument that the multichain model for $p p$ collisions [Cap80] predicted dominant contributions from two chains, one which led to fast produced particles, one to slow ones. The contributions from the projectile and target fireballs are indicated in Figure 6.2.

An interesting property of Equation 6.7 is the existence of three qualitatively different solutions for the cases i) $y_{0}<\sigma$, ii) $y_{0}=\sigma$, and iii) $y_{0}>\sigma$, respectively. For case i) the $d N / d y$ distribution will be peaked at midrapidity. For case ii) the distribution has a true plateau in the sense that its second derivative is zero $g^{\prime \prime}\left(y=y_{c m}\right)=0$. Case iii) allows a depression in the particle production to develop at midrapidity. The results of the symmetric Landau two-fireball fit to our data are consistent with case i), $y_{0}=.848 \pm .035, \sigma=.971 \pm .048$. This is a tighter fit to the data than the single Gaussian distribution, $\chi^{2} / \mathrm{NDF}=12.5 / 21$ compared to $\chi^{2} / \mathrm{NDF}=22.3 / 22$, but the two-fireball fit also introduces one additional parameter. It was impossible to fit the data with two, symmetric, isotropic fireballs. The estimates of the peak value of $d N / d y$, the width, and the integrated yield stated earlier were derived from the two-fireball fit. Differentiating between the one and two Landau fireball models requires a precise measurement near midrapidity and in the tail $y<1.0$ (including the region $y<0$ ), where our data is limited by acceptance.

The fully-corrected, participant proton $d N / d y$ distribution, deduced from the positive charge-excess, is shown in Figure 6.3 and compared to the distribution 


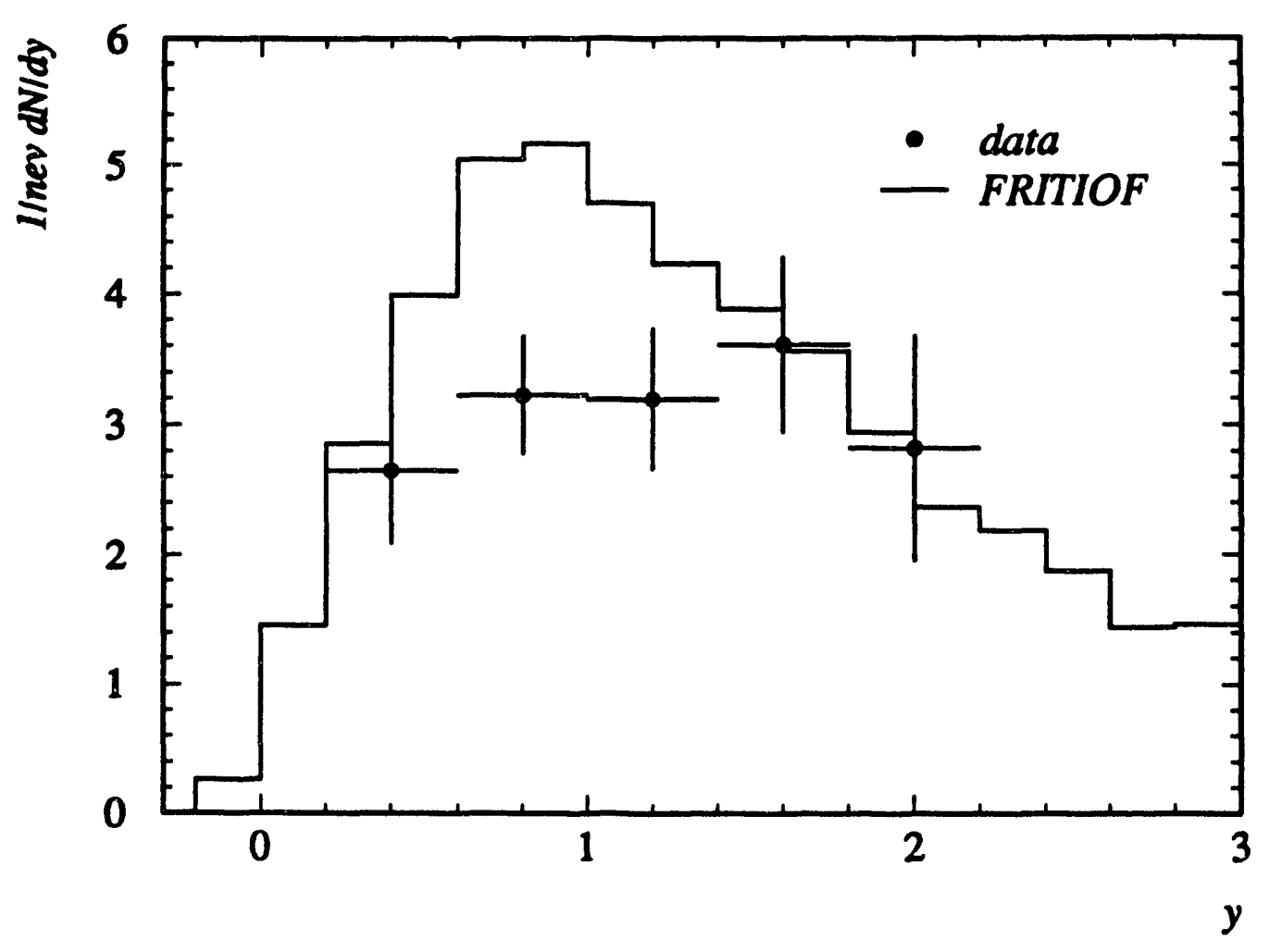

Figure 6.3: Corrected proton $d N / d y$ distribution. The data are compared to the predictions of FRITIOF. Only statistical errors are shown.

obtained from FRITIOF. A small contribution to the FRITIOF spectrum from produced protons is corrected for by subtracting the $d N / d y$ distribution of $\bar{p}$. See the footnote on page 154. This subtraction occurs naturally in the chargeexcess method. The FRITIOF histogram was calculated with the stopping parameter PAR(52) of Table 5.1 set to $\operatorname{PAR}(52)=10$ compared to the default value $\operatorname{PAR}(52)=1$. There may be a small number of target spectator protons which make it into our acceptance. The WA80 collaboration measured target remnants with $Z<3$ in the pseudorapidity range $-1.7<\eta<1.3$ from ${ }^{16} \mathrm{O}$ induced collisions on $\mathrm{C}, \mathrm{Cu}, \mathrm{Ag}$, and $\mathrm{Au}$ targets at 60 and $200 \mathrm{GeV} /$ nucleon [Alb88]. From the shape of the $d N / d \eta$ distribution for identified baryons from central collisions they inferred that target spectators could be dragged into the forward direction. From the integrated yield they concluded that enough energy is transferred from the projectile to target matter to completely disintegrate the target into light particles. And from the $\eta$ dependence of the baryon mean transverse momentum $\left\langle p_{T}\right\rangle$ they speculated that the rapidity shift $\Delta y=y-y_{\text {target }}$ could reach 0.5 units which exceeds the shift which could be ascribed to Fermi momentum within the nucleus. $^{3}$ This would only influence our measurement in the first bin. We assume that spectator contamination of our data is negligible and that the contribution to

\footnotetext{
${ }^{3}$ If the effect of exciting spectator matter were to liberate the bound nucleons at their Fermi momentum $p_{F}$ the maximum nucleon rapidity would be $y\left(p_{L}=p_{F} \simeq 270 \mathrm{MeV} / c, p_{T}=0\right) \approx$ 0.28 .
} 
the proton yield estimated from extrapolation of the data to low rapidity $y \lesssim 0.4$ can be attributed to participant protons. For a given impact parameter FRITIOF employs straight line geometry and a Woods-Saxon nucleon density distribution to determine which nucleons participate in binary collisions and the number of spectators [Nil86] but otherwise omits spectator nucleons completely. According to our FRITIOF calculation for $b<b_{\max }=2.5 \mathrm{fm}$, there will be approximately 8 spectator protons on average for ${ }^{32} S+{ }^{32} S$ interactions.

The data are consistent with either a flat distribution, a broad distribution peaked at $y \sim 1.5$ or even a distribution rising approximately linearly with rapidity. The proton data cannot be accounted for by FRITIOF which predicts a sharp peak at $y \sim 0.8$. The FRITIOF distribution obtained with the default parameter values exhibits even less stopping, with a sharper peak near target rapidity and a smaller yield at midrapidity. The data also rule out the parametrization [Kla85] $d N / d y \sim \cosh \left(y^{\prime}\right)$ suggested by FNAL bubble chamber measurements for $p p$ interactions at $205 \mathrm{GeV} / c$ [Whi74] of the proton inclusive cross section with respect to the center-of-mass momentum fraction $x=p_{L} / p_{0} \approx 2 m_{T} \sinh \left(y^{\prime}\right) / \sqrt{s}$ (Feynman $x$ ). Here $y^{\prime}$ is again the rapidity measured with respect to the center-of-mass and the hyperbolic cosine results from transforming $d \sigma / d x=$ constant to $d N / d y$. The FRITIOF spectrum forward of the peak at $y \approx 1$ is in rough, qualitative agreement with the hyperbolic cosine form.

From the relative flatness of the measured distribution one can obtain a crude estimate for the integrated proton yield $N_{p}=\int(d N / d y) d y \approx<d N / d y>\Delta y$. For $-0.2 \leq y<3.0, N_{p} \approx 3 \times 3.2=9.6$ protons compared to the FRITIOF yield $9.55 \pm .07$ protons. The missing peak is compensated by a higher yield at midrapidity and for $y \lesssim 0.4$. We obtain a more precise estimate of the yield in a model-dependent way below. For both the measured data and the simulation, the integrated yield does not equal the number of participant protons because of excitation of incident baryons into hyperon states. The correction for weak decays discussed in Chapter 5 removes a distortion to the $d N / d y$ spectrum but since the net charge from decays like $\Lambda \rightarrow p \pi^{-}$is strictly zero the yield cannot be recovered. According to our FRITIOF calculation, there are $\sim 5$ participant protons promoted to hyperons in the final state.

In order to extract the yield at midrapidity and the mean rapidity shift of participant protons, and to obtain a more quantitative estimate of $N_{p}$, we again employ the symmetric Landau two-fireball model of Equation 6.7. Das Gupta argued [Das78] that the strong angular anisotropy of the proton inclusive cross section from symmetric, light ion ${ }^{12} \mathrm{C}+{ }^{12} \mathrm{C}$ collisions at $800 \mathrm{MeV} /$ nucleon could be accounted for by a two-fireball model. He explicitly introduced nuclear transparencythe participating nucleons were slowed down but not completely stopped-and assumed that the excited nuclei came to thermal equilibrium before emitting particles isotropically in the rest frame of each fireball. A similar model was used by Klar and Hüfner [Kla85] to calculate the negative hadron rapidity distribution in $p p$ collisions at $200 \mathrm{GeV} / c$. Klar and Hüfner noted the resemblance of their model to that of Jacob and Slansky [Jac72] for hadron-hadron collisions in the 10 to $30 \mathrm{GeV} / c$ momentum range. Jacob and Slansky were able to reproduce fertures 
of inclusive cross sections usually attributed to multiperipheral models of multiparticle production by a calculation which emphasized diffractive excitation and fragmentation of the target and/or projectile. Another motivation for the twofireball fit is heuristic. It furnishes a simple way to subtract the contribution of overstopped projectile protons to the $d N / d y$ distribution in the target hemisphere $y<3.0$ so that we can estimate the mean rapidity shift $\langle\Delta y\rangle$ of participant target protons. An overstopped projectile(target) nucleon is defined as one which has suffered a rapidity shift $\Delta y>\left|y_{\text {beam }}-y_{\text {target }}\right| / 2$ so that it crosses midrapidity and is detected in the target(projectile) hemisphere. Overstopped target protons have been observed by Toothacker et al. [Too87] in $\bar{p}$ induced reactions on $\mathrm{Ag}$ nuclei.

The results of the two-fireball fit to the proton $d N / d y$ distribution are plotted in Figure 6.4. The dashed and dotted lines show the target and projectile fireball

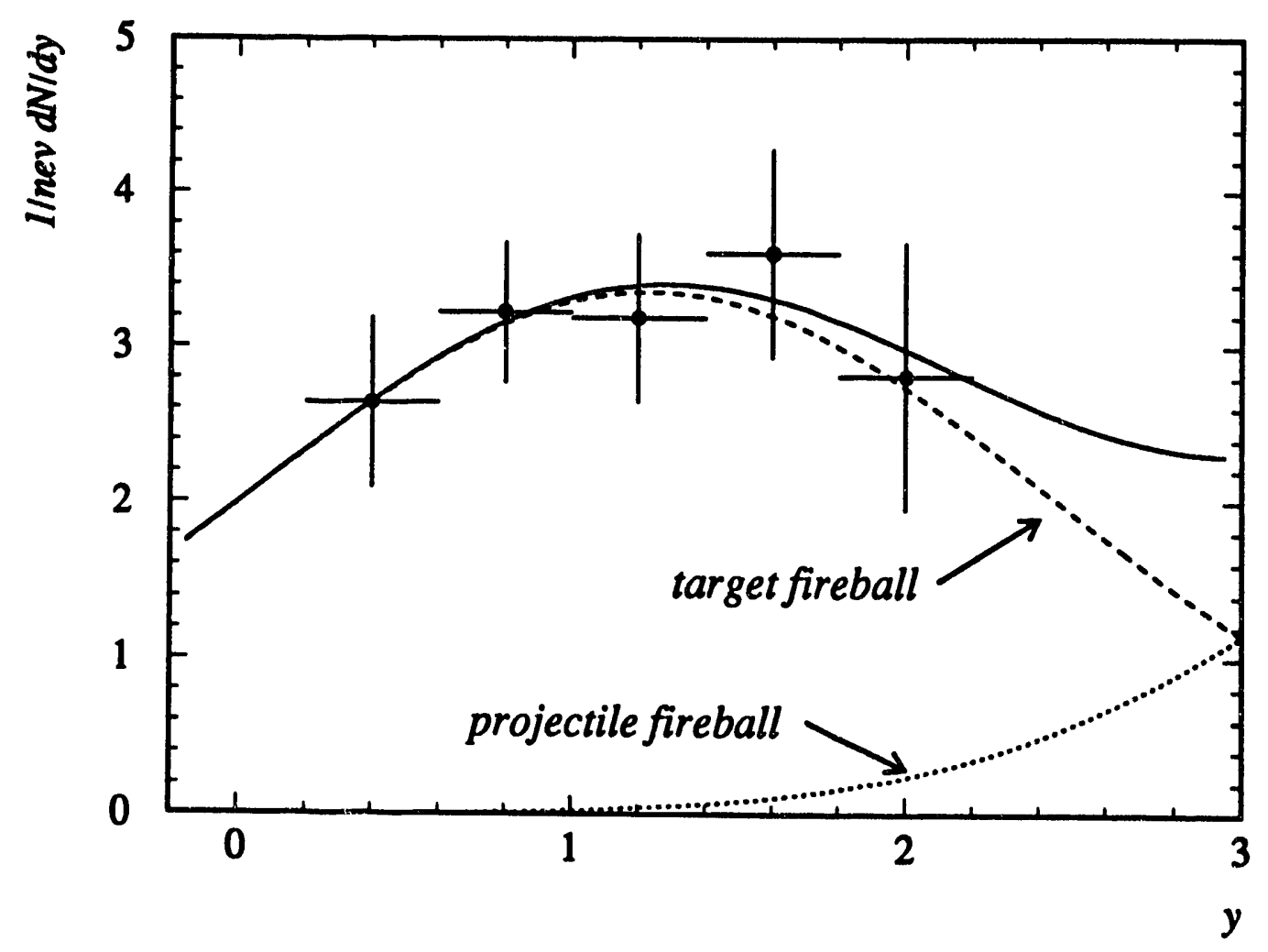

Figure 6.4: Landau two-fireball fit to the proton $d N / d y$ distribution. The dashed and dotted lines show the target and projectile contributions to the spectrum in the target hemisphere $y<3.0$.

contributions. The distance of the fireball peaks from midrapidity and the fireball width are $y_{0}=1.77 \pm 0.25$ and $\sigma=1.21 \pm 0.54$, respectively. Note that fitting Equation 6.7 to the data determines three parameters from five data points which have large statistical errors. We explored the stability of the fit by applying the same Monte Carlo procedure that was used for the negative hadron spectrum. The mean and rms values of the distributions of fitted parameters obtained from fits 
to a large ensemble of Monte Carlo spectra generated from our measurements are in good agreement with the parameter and error estimates from the uncertaintyweighted fit to the measured histogram. However, the shapes of the parameter distributions are skewed and the parameters are strongly correlated. The extrapolated values in the bins adjacent to the measurements are well-behaved, that is, their ensemble distributions are Gaussians with means equal to the best fit extrapolated values and widths consistent with the statistical errors on the measured data points. The more distant extrapolated values are not as well constrained. For example, the ensemble distribution of the extrapolated point at midrapidity is very broad and exhibits two peaks, one at $d N / d y \sim 1$, the other at $d N / d y \sim 3$. The mean value is in good agreement with the one extrapolated from the best fit, $d N / d y(y=3)=2.3 \pm 1.3$ protons per unit of rapidity where the assigned statistical error is the rms deviation of the ensemble of extrapolations to midrapidity.

The integrated yield of target protons for $-3.0 \leq y<3.0$, which includes the full tail of the target fireball, is $N_{p}=10.2 \pm 2.0 \pm 1.3$. All results from our fits to the rapidity distributions of protons and negative hadrons are collected in Table 6.1. Because of the symmetry of Equation 6.7, the overstopped target protons

\begin{tabular}{||lcccc||}
\hline \hline Observable & \multicolumn{3}{c||}{ Fit parameters } & $\langle N\rangle$ \\
\hline & \multicolumn{4}{c}{} \\
Equation 6.3 & $A$ & & $\sigma$ & \\
& $21.64 \pm 0.46$ & & $1.376 \pm .025$ & $36.23 \pm 0.52$ \\
Equation 6.7 & $A$ & $y_{0}$ & $\sigma$ & \\
& $14.72 \pm 0.62$ & $0.848 \pm .035$ & $0.971 \pm .048$ & $35.31 \pm 0.59$ \\
$A A \rightarrow p X$ & & & & \\
& $3.36 \pm 0.53$ & $1.77 \pm 0.25$ & $1.21 \pm 0.54$ & $10.2 \pm 2.0 \pm 1.3$ \\
\hline \hline
\end{tabular}

Table 6.1: Summary of the fits to proton and negative hadron $d N / d y$ distributions. $A, y_{0}$, and $\sigma$ are the normalization, the position, and the width of the Landau fireballs. For the two-fireball model $y_{0}$ is the distance from midrapidity, for the single-fireball model $y_{0} \equiv 3.0$ by symmetry. $\langle N\rangle$ is the average number of negative hadrons or protons in the target hemisphere in rapidity.

with $y>3$ are compensated by the overstopped projectile protons. The first error is statistical, estimated by the rms deviation of the ensemble of integrated values. The second error is systematic and reflects the dependence of $N_{p}$ on the lower bound of integration $y_{L}$. For $y_{L}=-1.0$ and $-0.2, N_{p}=9.8$ and 8.9 protons, respectively. There is no evidence in the sparse available data for leading protons ${ }^{4}$ in high energy $p A$ [Too87], [Abe88] or $\alpha \alpha$ and $d d$ [Bel86] for fragmentation pro-

\footnotetext{
${ }^{4}$ Note that in equal mass $A A$ collisions the projectile and target fragmentation regions are equivalent. Leading particles refers to particles with rapidity close to either the beam or target. Most of the available data from $p A$ collisions, which has usually been obtained in fixed-target experiments, is for the projectile fragmentation region.
} 
tons beyond the kinematical limit $y_{\max }=y_{\text {beam. }}$. Fermi motion up to $p_{L} \sim 270$ $\mathrm{MeV} / c$ can only account for an excess of $\sim 0.3$ units of rapidity beyond $y_{\text {max }}$. However, a mechanism which might drive participant nucleons beyond the kinematical limit-final state rescattering which is incorporated into hydrodynamic models as predominantly longitudinal pressure in compressed nuclear matter-cannot occur for $p A$ collisions in the projectile fragmentation region. There simply isn't any comoving excited matter. The rescattering mechanism can explain large rapidity shifts to kinematically forbidden values. For example, a $1 \mathrm{GeV} / c$ proton at $y=0$ can undergo elastic or quasi-elastic scattering and freeze out with a final rapidity of $y \approx-1$. A measurement of identified leading protons beyond $y_{\max }$ in $A A$ collisions would be important to test the validity of this interpretation.

To calculate the number of participant protons from the observed integrated yield $N_{p}$ we assume that the missing protons can be identified with half of the excess of hyperons $Y$ over anti-hy erons $\bar{Y}$. From the ratio of the inclusive cross sections for $\Sigma^{ \pm}$and $\Lambda\left(\Sigma^{0}\right)$ production Wróblewski [Wro85] established the empirical rule $\left.\langle Y\rangle_{N N}=1.6 \pm 0.1<\Lambda+\Sigma^{0}\right\rangle_{N N}$. Isospin averaged values of hadron production appropriate for generic $N N$ collisions, including the mean multiplicities $\left\langle\Lambda+\Sigma^{0}\right\rangle_{N N}$ and $\left\langle\bar{\Lambda}+\bar{\Sigma}^{0}\right\rangle_{N N}$, have been calculated from minimum bias $p p$ and $n p$ interactions at $200 \mathrm{GeV} / c$ in the compilation of Gazdzicki and Hansen [Gaz91]. For $A A$ collisions we assume

$$
\begin{aligned}
\langle Y-\bar{Y}\rangle_{A A} & =\text { (the number of participant } N N \text { pairs })<Y-\bar{Y}\rangle_{N N} \lambda \\
& =\left(2 N_{p}+\frac{1}{2}<Y-\bar{Y}>_{A A}\right)<Y-\bar{Y}>_{N N} \lambda
\end{aligned}
$$

In Equation 6.9, the number of participant $N N$ pairs scales the $N N$ result to isoscalar $A A$ interactions in a way that incorporates the impact parameter selection of our trigger and $\lambda$ is an experimentally determined number which introduces the enhanced strangeness production observed by NA35 in central ${ }^{32} \mathrm{~S}+{ }^{32} \mathrm{~S}$ collisions [Bar90]. The factor $2 N_{p}$ accounts for the participant neutrons. The factor $1 / 2$ is required because $\langle Y-\bar{Y}\rangle_{A A}$ is the total multiplicity half of which, on average, can be associated with nucleon-nucleon pairs. Equation 6.9 can easily be solved to yield

$$
<Y-\bar{Y}>_{A A}=\frac{2 N_{p}<Y-\bar{Y}>_{N N} \lambda}{1-\frac{1}{2}<Y-\bar{Y}>_{N N} \lambda} .
$$

From the values in Gazdzicki and Hansen $\left\langle\Lambda+\Sigma^{0}\right\rangle_{N N}=0.096 \pm 0.015$ and $\left\langle\bar{\Lambda}+\bar{\Sigma}^{0}\right\rangle_{N N}=0.013 \pm 0.005$, and the NA35 result $\lambda \approx 2,\langle Y-\bar{Y}\rangle_{A A}=6.2 \pm 2.4$, assuming an uncertainty of $20 \%$ for the measured strangeness enhancement $\lambda$. Dividing by four to recover the yield in iarget protons we obtain a correction factor of $1.6 \pm 0.6$. Our best estimate of the number of participant protons is $11.8 \pm 2.1$ neglecting the possible systematic error in $N_{p}$.

From the negative hadron yield and the farticipant proton number in half of phase space $y<3$ the mean negative hadron multiplicity per $N N$ pair in ${ }^{32} \mathrm{~S}+{ }^{32} \mathrm{~S}$ interactions over full phase space is $\left\langle N_{h_{-}}\right\rangle=2.99 \pm 0.53$ charged particles. This is to be compared with the isospin averaged value from $N N$ of $3.22 \pm 0.06$. The 
numbers agree within statistical errors. This suggests the absence of intranuclear cascading in the sense of multiple, binary $N N$ collisions between projectile and target nucleons during the interpenetration phase of the collision. DeMarzo et al. [DeM82a] noted that the large values of the particle production ratio $R(y)$ of Equation 6.8 in the target rapidity hemisphere for $p A r$ and $p X e$ reactions came mainly from events with large values of the number of struck target protons ${ }^{5}$ with momenta $150<p<600 \mathrm{MeV} / c$, identified in the streamer chamber from ionization. Apparently, the minimum bias triggers generally employed by $p A$ experiments are more successful at selecting events in which the incident proton(s) has(have) undergone multiple binary collisions than the $E_{V \text { eto }}, E_{T}$ and charged multiplicity triggers used to select central events in $A A$, particularly in collisions of light symmetric nuclei. Daté, Gyulassy and Sumiyoshi [Dat85] have pointed out that this problem, which they attributed to the large contributions of interactions near the nuclear surfaces, is likely to persist in symmetric collisions even for the heaviest nuclei. Final state rescattering is unlikely to produce particles because of the relatively low available energies in the two-particle centers-of-mass. Gavin and Ruuskanen [Gav91] noted that $\pi \pi$ interactions at $\sqrt{s_{\pi \pi}}<1 \mathrm{GeV}$ are predominantly elastic and used this fact to justify their assumption that pion number was conserved during the evolution toward freezeout of the pion fluid produced in ${ }^{32} \mathrm{~S}+{ }^{32} \mathrm{~S}$ collisions at $200 \mathrm{GeV} / c$.

The mean rapidity shift of participant target protons, after subtraction of overstopped projectile protons is $\langle\Delta y\rangle=3.0-y_{0}=1.23 \pm 0.25$. As for the integrated yield, $\langle\Delta y\rangle$ depends on $y_{L}$. The mean rapidity shift for $y_{L}=-2.0,-1.0$, and -0.2 is $\langle\Delta y\rangle=1.25,1.32$, and 1.50, respectively. Bell et al. [Bel86] measured semi-inclusive proton rapidity distributions by the charge-excess method for different windows of charged multiplicity in $\alpha \alpha$ and $d d$ collisions at $\sqrt{s_{N N}}=31.2 \mathrm{GeV}$ at the ISR. For $\alpha \alpha$ they reported $\langle\Delta y\rangle=1.03,1.26$, and 1.42 for the multiplicity bins $3 \leq n_{c h}<7,9 \leq n_{c h}<18$, and $25 \leq n_{c h}$, respectively. We estimate the charged multiplicity which would correspond to our data by

$$
n_{c h} \sim\left(\left[<N_{h_{-}}>\times 2 \times 2\right]+1\right) \times<w>\approx 22 .
$$

The first factor of 2 adds the positively charged mesons, the second extends the result to full phase space, and one charged nucleon is the isospin average per $N N$ collision. $\langle w\rangle \equiv A \sigma_{p A} / \sigma_{A A}=1.7$ is the average number of wounded nucleons determined by Bell et al., which we identify with the number of participant $N N$ pairs since $\langle w\rangle=1.0$ for $N N$ collisions. The $d N / d y$ distribution for $n_{c h} \geq 25$ most resembles our data in shape with a peak at $\Delta y \approx 1.25$ and a proton yield of about $1 / 3$ peak value at $\Delta y=3$. However, the ISR data appears to fall more abruptly as $\Delta y \rightarrow 0$ although this data also lacks a point at $\Delta y=0$. From the qualitative agreement in shape and in the mean rapidity shift for $y_{L}=-0.2$ we conclude that the nuclear matter stopping power is about the same for central ${ }^{32} \mathrm{~S}+{ }^{32} \mathrm{~S}$ and $\alpha \alpha$ collisions at comparable $\sqrt{s}$. A similar calculation of the negative

\footnotetext{
${ }^{5}$ Evaporation protons, which result from the de-excitation of the nucleus after the end of the projectile interaction, have low momentum $p<200 \mathrm{MeV} / \mathrm{c}$ and were assumed to stop in the target material [DeM82a].
} 
charged multiplicity $n_{-} \sim 2<N_{h_{-}}>\times<w>$ indicates that the appropriate semi-inclusive negative rapidity distributions from $p p$ and $\alpha \alpha$ to compare to our data have $n_{-} \sim 6$ and $\sim 10$, respectively.

Toothacker et al. [Too87] measured identified proton $d N / d y$ distributions in the projectile fragmentation region for $p$ induced reactions on $\mathrm{Mg}, \mathrm{Ag}$ and $\mathrm{Au}$ targets at $100 \mathrm{GeV} / c$. For protons with momenta $14<p<100 \mathrm{GeV} / c$, which corresponds to rapidity shifts from $\Delta y=3$ to 0 , they obtained average values for fuily inclusive measurements of $\langle\Delta y\rangle=1.04 \pm 0.25,1.24 \pm 0.13$, and $1.07 \pm 0.16$ for $\mathrm{Mg}, \mathrm{Ag}$ and $A u$, respectively. ${ }^{6}$ By combining their data into a single $p A$ data set they were able to plot separately the $d N / d y$ distributions for events with fewer than 3 (peripheral collisions) or 3 or more associated slow protons (central collisions). Both the peripheral and central collision data were shown to agree, within large statistical errors, with predictions from the stopping power model developed by Busza and Goldhaber [Bus84], implying the observation of $\langle\Delta y\rangle \sim 2.5$ in central $p A$ collisions. Abe et al. made similar measurements of the $d N / d y$ distribution of the leading positively charged particle in $p$ induced reactions at $200 \mathrm{GeV} / c$. For their fully inclusive data they observed $\langle\Delta y\rangle=1.4,1.8,2.0$, and 2.0 for $p, \mathrm{Mg}$, $\mathrm{Ag}$, and $\mathrm{Au}$ targets, respectively. For their most central semi-inclusive data set, selected by requiring greater than $\sim 3$ recoil target protons and a negative charged multiplicity greater than 12 , they measured $\langle\Delta y\rangle=2.4$. By estimating the effect of events in which the leading baryon was an unobserved neutron, they obtained a lower limit of $\langle\Delta y\rangle=2.0$ for central $p A$ collisions. The nuclear stopping power in central ${ }^{32} \mathrm{~S}+{ }^{32} \mathrm{~S}$ collisions, deduced from the mean rapidity shift, is significantly lower than that which has been observed in central $p A$ collisions but is consistent with that observed in the most central subset of symmetric, light $A A$ interactions at the ISR.

The picture of light nucleus-nucleus collisions at $200 \mathrm{GeV} / c$ that emerges from the rapidity distributions of incident protons and produced particles is similar to the one advocated by Anishetty, Koehler and McLerran [Ani80] for central collisions between heavy nuclei at $E_{c m} \sim 50 \mathrm{GeV} /$ nucleon. They argued for the formation of thermally equilibrated fireballs in the nuclear fragmentation regions at $y \sim 1.3$ units of rapidity from the original rest frames of the incident nuclei. As the interacting nuclei pass through each other, the participant nucleons are excited as if they undergo only $1-2$ nucleon-nucleon collisions. The excited nucleons fragment and emit particles. Virtually all of the final state nucleons and some fraction of the produced particles-the fraction depending on the momenta of the produced particles, the $\gamma$ factor of the comoving nucleons and the size of the Lorentz-contracted nuclei-remain trapped within the nuclear volume. Rescattering in participant nuclear matter drives the system toward thermal equilibrium. The fireballs expand hydrodynamically until freezeout.

In this picture, the initial conditions for hydrodynamic expansion are interme-

\footnotetext{
${ }^{6}$ The absence of any systematic $A$ dependence of $\langle\Delta y\rangle$ from $\mathrm{Mg}(A=24)$ to $\operatorname{Au}(A=197)$ is puzzling. Ref. Too87 does not describe event selection for the fully inclusive data. Comparing this data to the $p p$ estimate obtained by Busza and Goldhaber [Bus84], $\langle\Delta y\rangle=1$, suggests that the fully inclusive $p A$ data is dominated by peripheral collisions.
} 
diate between those suggested by Landau, who assumed complete stopping with all incident particles coming to rest in the center-of-mass and all particle production occurring within the Lorentz-contracted nuclear volume, and those employed by Bjorken, who proposed that most particle production occurs outside of widely separated projectile and target fragmentation regions. Daté, Gyulassy and Sumiyoshi [Dat85] estimated that the stopping domain of nuclear collisions, defined as the energy range over which the Landau initial conditions provide the correct description, extends only up to $E_{c m} \sim 5 \mathrm{GeV} /$ nucleon. Bjorken's initial conditions were inferred from the existence of a central plateau [Bjo83] for the inclusive particle production as a function of rapidity for ISR and SP $\bar{P} S$ collider data at $E_{c m} \sim 30-270$ $\mathrm{GeV} /$ nucleon. So it is not surprising that at $\sqrt{s}=20 \mathrm{GeV}$, which corresponds to bombarding energies of $E_{c m}=10 \mathrm{GeV} /$ nucleon, we exceed the stopping domain but are far from the Bjorken limit. With the $\sim 2-3$ units of rapidity width of the nuclear fragmentation region suggested by our data and a total rapidity gap of $y_{\text {beam }}-y_{\text {target }}=6.0$, there is no phase space for a baryon-free central region to develop. The implication of this result for the upcoming $\mathrm{Pb}$-beams program at CERN, which will accelerate the heaviest nuclei to $p \sim 190 \mathrm{GeV} / c$ in fixed-target mode, is that the midrapidity region is likely to be baryon-rich rather than baryonfree. At RHIC, where the rapidity gap will be 10.8 units of rapidity, only a small interval of rapidity is likely to be baryon-free in central ${ }^{197} \mathrm{Au}+{ }^{197} \mathrm{Au}$ collisions. ${ }^{7}$

\subsection{Transverse Momentum Spectra}

In this section we present fully corrected, inclusive transverse momentum distributions for negative hadrons and protons. The distributions are obtained by integrating the Lorentz invariant cross section over rapidity,

$$
E \frac{d^{3} \sigma}{d p^{3}} \propto \frac{1}{p_{T}} \frac{d^{2} N}{d y d p_{T}} \rightarrow \frac{1}{p_{T}} \frac{d N}{d p_{T}} .
$$

For negative hadrons, our rapidity acceptance is large enough to be subdivided in order to separate midrapidity from target rapidity effects and to explore the factorization hypothesis of Equation 6.3. For small rapidity intervals $\Delta y \sim 1$ unit of rapidity, $1 / p_{T} d N / d p_{T}$ is equivalent to an unnormalized invariant cross section so we can compare the shapes of our spectra to results obtained from spectrometer experiments for $p p$ and $A A$ collisions at comparable energies. We find no evidence for a low- $p_{T}$ enhancement relative to $p p$, although the negative hadron spectra possess a two-component or concave structure and rise for $p_{T} \lesssim 0.400 \mathrm{GeV} / c$. Both the negative hadron and proton distributions can be accommodated within a simple thermal model at a common temperature $T \simeq 170 \mathrm{MeV}$ if we identify the increase at low $p_{T}$ with pions originating from resonance decay.

\footnotetext{
${ }^{7}$ Preliminary FRITIOF calculations performed by M. Gyulassy [Gyu91] using our choice for the stopping parameter $\operatorname{PAR}(52)=10$, which led to less stopping than shown by our data for ${ }^{32} \mathrm{~S}+{ }^{32} \mathrm{~S}$, exhibited exactly these features for $\mathrm{Pb}+\mathrm{Pb}$ collisions at the SPS and $\mathrm{Au}+\mathrm{Au}$ at RHIC.
} 
The negative hadron $p_{T}$ spectra $1 / p_{T} d N / d p_{T}$ as a function of $p_{T}$ are shown in Figure 6.5 for our full acceptance $0.6<y<2.8$, and for the rapidity intervals $1.0<y<2.0$ and $2.0<y<2.8$. The region $2.0<y<2.8$ is the $\sim$ unit of rapidity nearest midrapidity that we can measure and coincides with the domain over which the rapidity distribution $d N / d y$ is fairly flat. The HELIOS collaboration [Ake90] has measured inclusive negative particle $p_{T}$ spectra for $1.0<y<1.9$ in $p A$ and asymmetric $A A$ collisions at $200 \mathrm{GeV} /$ nucleon. All three spectra shown in Figure 6.5 have the same qualitative features-they turn upward softly at $p_{T} \sim 0.400$ $\mathrm{GeV} / c$, they flatten and start to turn over as $p_{T} \rightarrow 0$, and they start to flatten again at high $p_{T} \sim 1.5 \mathrm{GeV} / c$. The similarity of the spectra for different rapidity intervals, which will be quantified below when we fit the data, provides evidence that the factorization hypothesis is approximately satisfied. Together, factorization and the broad $d N / d y$ distribution imply that pure thermal models or models which introduce spherically symmetric rather than mainly longitudinal dynamics will fail to describe the data. The observation of $p_{T}$-dependent structure underscores the importance of a broad $p_{T}$ acceptance in understanding the dynamical origins of the spectra. We plot the data as a function of $p_{T}$ rather than the transverse mass $m_{T}=\sqrt{p_{T}^{2}+m^{2}}$ so that the transverse momentum acceptance is not obscured.

In hadron collisions at ISR energies, the transverse momentum distributions of charged particles at fixed laboratory rapidities have been observed to be approximately exponential,

$$
f\left(p_{T}\right)=\left.E \frac{d^{3} \sigma}{d p^{3}}\right|_{y=\text { const }} \sim A \exp \left(-b p_{T}\right),
$$

for $0.2<p_{T}<1.4 \mathrm{GeV} / c$, with slope parameters $b$ decreasing with increasing mass of the detected particle [Gia79]. It has been pointed out [Kin78] that the different mass hadrons can be described by a single universal function of $\boldsymbol{m}_{T}$

$$
f\left(p_{T}\right)=A \exp \left(-b m_{T}\right)
$$

with a slope $b \sim 6$. Plotted as a function of $p_{T}$, the exponential in transverse mass tu:ns over as $p_{T} \rightarrow 0$, that is, its slope goes to zero $f^{\prime}\left(p_{T}=0\right)=0$. Guettler et al. measured the inclusive production of low momentum charged pions with $.045<p_{T}<.400 \mathrm{GeV} / c$ at $y=0$ [Gue76] and observed a turnover at very low $p_{T}$ which could not be reproduced by an exponential in $p_{T}$ but could be well fit by Equation 6.13 with $b \simeq 7$. Much earlier, Imaeda [Ima67] noted that the transverse momentum distributions of both baryons and mesons from collisions at incident momenta $p \sim 5-25 \mathrm{GeV} / c$ could be well fit by the expression

$$
f\left(p_{T}\right)=\frac{1}{p_{T}} \frac{d N}{d p_{T}}=A m_{T} K_{1}\left(m_{T} / T\right),
$$

where $K_{1}$ is a modified Bessel function. For $m_{T} / T$ large (greater than $\sim 3$ ) this can be approximated by

$$
f\left(p_{T}\right)=\frac{1}{p_{T}} \frac{d N}{d p_{T}}=A \sqrt{m_{T}} \exp \left(-m_{T} / T\right)
$$




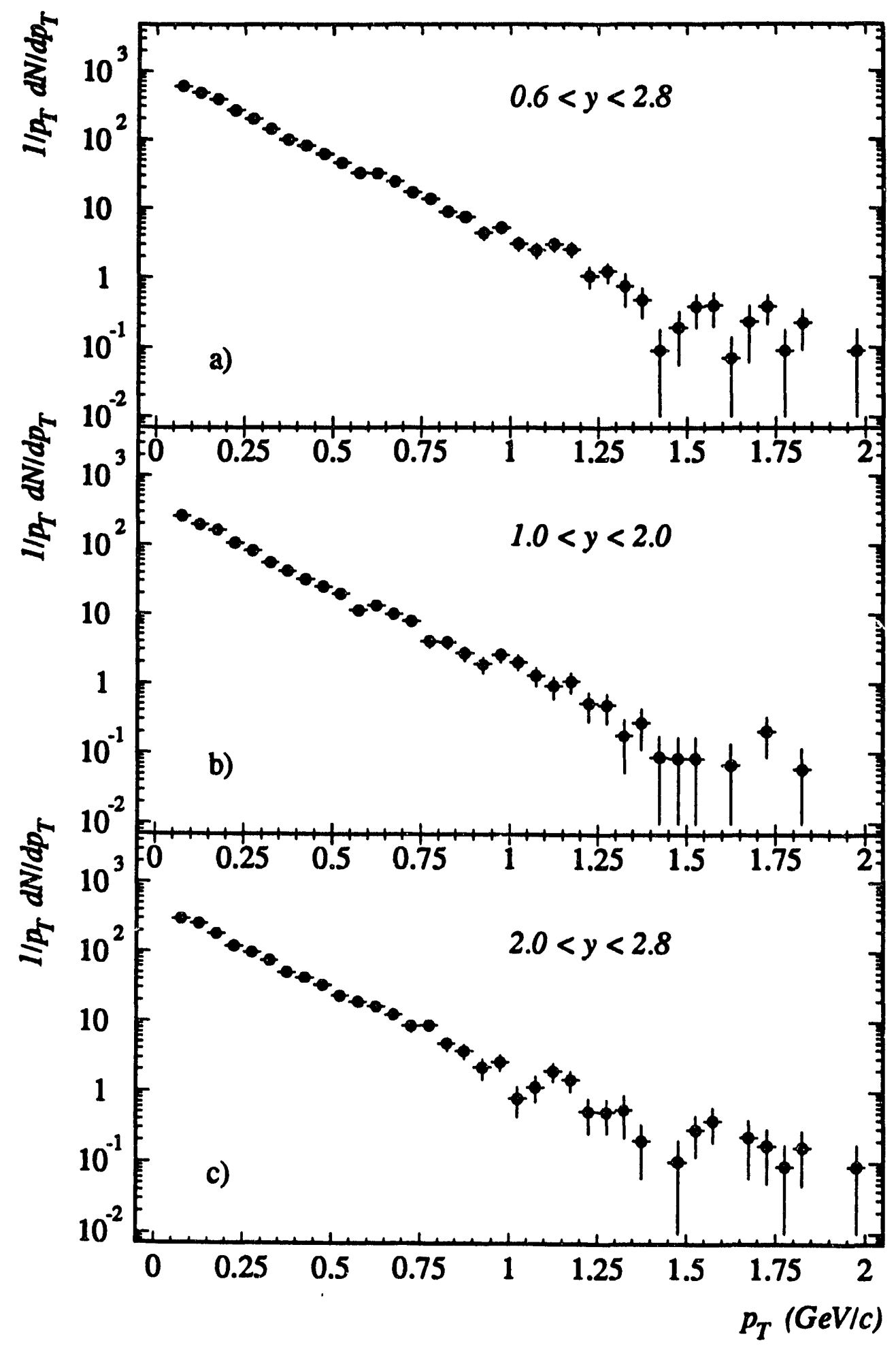

Figure 6.5: Tully corrected negative hadron $p_{T}$ distributions $1 / p_{T} d N / d p_{T}$ for the rapidity intervals a) $0.6<y<2.8$, b) $1.0<y<2.0$, and c) $2.0<y<2.8$. Only statistical errors are shown. 
Imaeda concluded that "secondary particles were produced through states of thermal equilibrium of final-state interaction", regardless of the production process, hence the replacement of the slope parameter with the more physical temperature. Hagedorn derived Equation 6.14 from his statistical thermodynamics of strong interactions [Hag65] and has since argued [Hag83] that no evidence contradicts the assertion that the inclusive $p_{T}$ distributions below $p_{T} \sim 1 \mathrm{GeV} / c$ are thermal.

In thermodynamic equilibrium at temperature $T$ and chemical potential $\mu$ the number of particles of a given species per unit volume in momentum space is

$$
\frac{d^{3} N}{d p^{3}}=A \frac{1}{\exp [(E-\mu) / T] \pm 1}
$$

which leads to the following expression for the invariant cross section

$$
\frac{1}{p_{T}} \frac{d^{2} N}{d y d p_{T}}=A \frac{m_{T} \cosh \left(y-y_{0}\right)}{\exp \left[\left(m_{T} \cosh \left(y-y_{0}\right)-\mu\right) / T\right] \pm 1} .
$$

We have used the relativistic expression $E=m_{T} \cosh \left(y-y_{0}\right)$ and have explicitly introduced the fireball rapidity $y_{0}$. In the Maxwell-Boltzmann limit ${ }^{8}$ this reduces to

$$
\frac{1}{p_{T}} \frac{d^{2} N}{d y d p_{T}}=A m_{T} \cosh \left(y-y_{0}\right) \exp \left[-m_{T} \cosh \left(y-y_{0}\right) / T\right]
$$

which for a small rapidity interval near fireball rapidity becomes

$$
f\left(p_{T}\right)=\left.\frac{1}{p_{T}} \frac{d^{2} N}{d y d p_{T}}\right|_{y=y_{0}}=A m_{T} \exp \left(-m_{T} / T\right) .
$$

Integrating Equation 6.18 over rapidity recovers Equation 6.14. The reason for introducing the different expressions above is that the negative hadron spectra can be equally well fit by two-component superpositions of any of Equations 6.13, 6.14, 6.15 , and 6.19,

$$
g\left(p_{T}, A_{1}, A_{2}, T_{1}, T_{2}\right)=f\left(p_{T}, A_{1}, T_{1}\right)+f\left(p_{T}, A_{2}, T_{2}\right) .
$$

In order to estimate a physical temperature one must judge which expression brst reflects the underlying dynamics.

In Figure 6.6 we show that the negative hadron $p_{T}$ spectrum cannot be accounted for by a single-component thermal distribution. The solid lines are fits to an exponential in transverse mass over our full $p_{T}$ acceptance $0.050<p_{T}<2.0$ $\mathrm{GeV} / c$. The fits are poor. systematically below the data at both high and low transverse momentum. A single exponential in $p_{T}$ with a slope parameter $b \simeq 5.5$ fits the cata but the fit ignores important structure in the spectra.

Although no single-component purely thermal model can account for the spectra over the full $p_{T}$ range, over a restricted range the spectra can be fit with a

\footnotetext{
${ }^{8}$ In the Maxwell-Boltzmann limit the chemical potentials play a role in determining the relative abundances of particle species and in embodying conservation laws for baryon number and strangeness [Sol90] but are absorbed in the normalization for single particle inclusive spectra.
} 


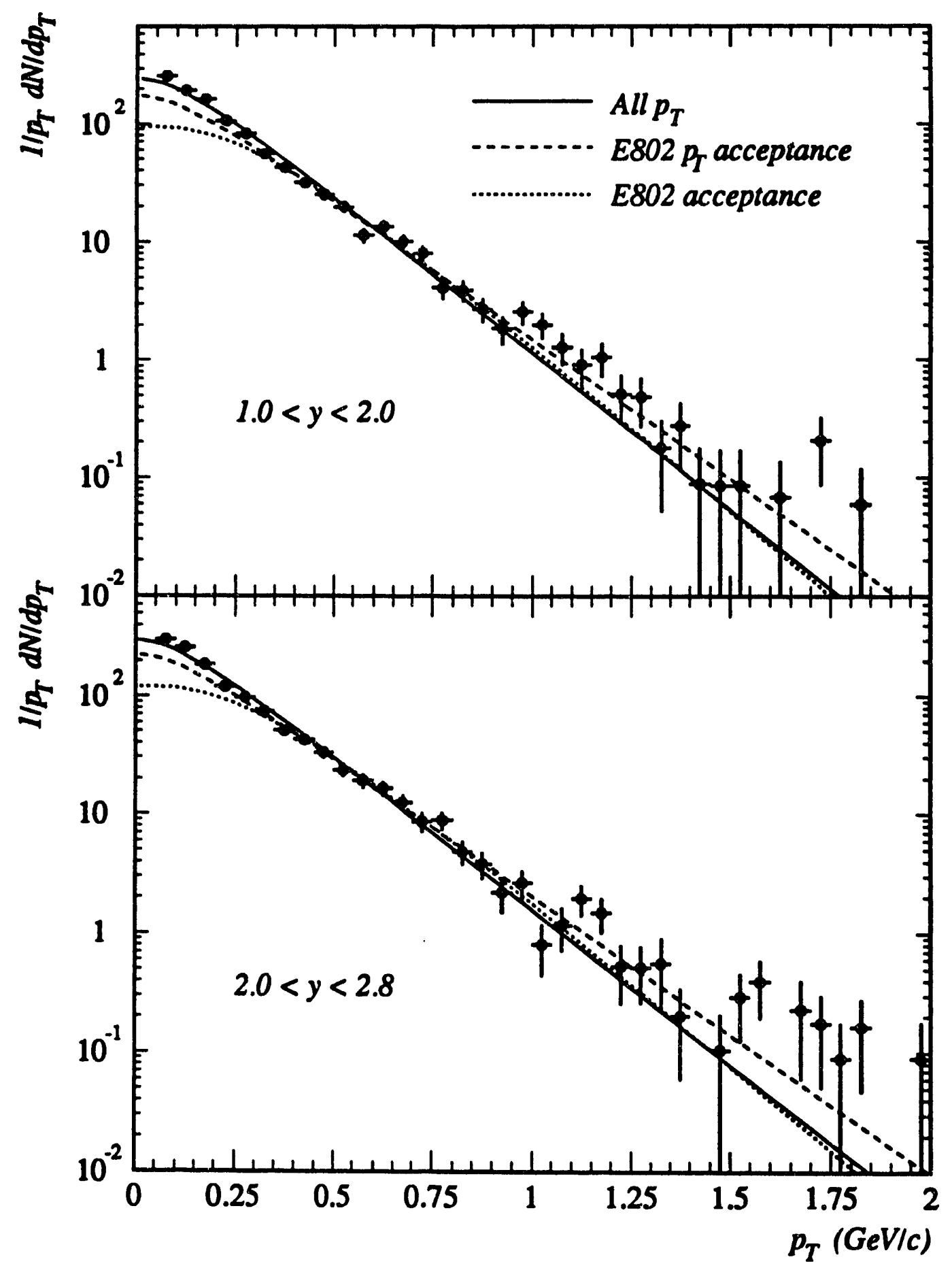

Figure 6.6: Single-component thermal fits to negative hadron $p_{T}$ distributions. Two different rapidity intervals are shown. The solid lines are fits to Equation 6.13, an exponential in transverse mass, over our full $p_{T}$ acceptance. The dashed and dotted lines are fits to Equations 6.13 and 6.19, the midrapidity Boltzmann form, over the acceptance of AGS experiment E802, $0.300<p_{T}<1.0 \mathrm{GeV} / c$. 
single component. The E802 collaboration has measured the transverse momentum and rapidity distributions of $\pi^{ \pm}, K^{ \pm}$and $p$ from central collisions of $14.6 \mathrm{~A}$ $\mathrm{GeV} / \mathrm{c}^{28} \mathrm{Si}$ projectiles with ${ }^{197} \mathrm{Au}$ nuclei [Abb90] and obtained good fits to the $\pi$ and $K$ data with either exponentials in transverse mass or Boltzmann distributions. For comparison we fit our spectra over their $p_{T}$ acceptance $0.300<p_{T}<1.0$ $\mathrm{GeV} / c$. The dashed lines are extrapolations of the fits to Equation 6.13, the exponential in the transverse mass. The dotted lines are extrapolations of the fits to the midrapidity Boltzmann form $m_{T} \exp \left(-m_{T} / T\right)$, Equation 6.19. The values of the temperature parameters $T_{0}$ and $T_{B}$ obtained for the exponential and Boltzmann fits are $T_{0}=182 \pm 9$ and $T_{B}=138 \pm 6 \mathrm{MeV}, T_{0}=185 \pm 9$ and $T_{B}=140 \pm 7$ $\mathrm{MeV}$ for the respective rapidity intervals $1.0<y<2.0$ and $2.0<y<2.8$. The errors are statistical only. These numbers are somewhat larger than the $\pi^{-}$results reported by $\mathrm{E} 802$ in a small rapidity interval $1.2<y<1.4$ close to the geometrical center of mass ${ }^{9}$ for central $\mathrm{Si}+\mathrm{Au}, T_{0}=161 \pm 10$ and $T_{B}=126 \pm 10 \mathrm{MeV}$. The increase in temperature from AGS to SPS energy is consistent with the well-known slow increase of $\left\langle p_{T}\right\rangle$ with $\sqrt{s}$. The Boltzmann form is artificial, even for the restricted acceptance, in spite of its virtue of imparting to $T_{B}$ a specific meaning and implying a specific dynamics. It requires a curvature in the spectra that is not substantiated by the data. The yield for $p_{T}<0.300 \mathrm{GeV} / c$ in excess of the extrapolations from the single-component fits may account for the relatively small number of $\pi^{-}$observed by $\mathrm{E} 802$ at midrapidity, as evidenced by the inability of Chapman and Gyulassy [Cha91] to find a model for the E802 data which obtained a consistent normalization of protons and pions at the same time. Stachel pointed out [Sta91] that a recent result from the E810 experiment at the AGS [Lov91], which has the same shape as the E802 data for $p_{T}>0.300$, reveals a clear rise above the exponential in transverse mass for $p_{T}<0.300 \mathrm{GeV} / c$.

A two-component structure or an increased yield relative to an exponential in $m_{T}$ are insufficient evidence for the existence of a low- $p_{T}$ enhancement. The observation of the two-component structure of the pion $p_{T}$ distribution, alternatively described as a concave shape, had already been made for measurements of the invariant cross section in $p p$ collisions at the ISR [Alp75]. One must observe a systematic effect in the ratio of the transverse momentum distribution from $A A$ collisions to an appropriate comparison distribution from $p p$ interactions at comparable energy. Defining the comparison spectrum is problematic unless both the $p p$ and $A A$ distributions have been measured by the same experiment because of inconsistencies in acceptance, efficiency and the corrections which may have been applied to the data. NA35 observed a low- $p_{T}$ enhancement for negative hadrons produced near midrapidity in $p+A u$ and ${ }^{16} \mathrm{O}+\mathrm{Au}$ collisions at $200 \mathrm{GeV}$ /nucleon relative to unpublished NA5 $p p$ data at $200 \mathrm{GeV} / c$ [Str88]. The NA35 result was corrected for electrons but; not for weak decays close to the target or secondary interactions. A stronger eflect was reported by the HELIOS collaboration [Ake90], CERN experiment NA34, who compared their data from $200 \mathrm{GeV} /$ nucleon $p+W$,

\footnotetext{
${ }^{9}$ In $A A$ collisions of different mass nuclei, for a given impact parameter the geometrical center of mass can be calculated frorn the number of participant target nucleons swept out by the overlapping projectile. For exarnple in $a b=0$ collision ${ }^{28} \mathrm{Si}$ sweeps out $\sim 75$ Au nucleons.
} 
${ }^{16} \mathrm{O}+\mathrm{W}$, and ${ }^{32} \mathrm{~S}+\mathrm{W}$ collisions for $1.0<y<1.9$ to a parametrization of the ISR results of Ref. Alp75. However, the NA34 data had also not been corrected for negative hadrons resulting from weak decays of $K_{0}^{p}$ and $\Lambda$ and from hadrons produced in secondary collisions [Jac91b]. Experiment E810 at the AGS plotted the invariant cross section as a function of transverse mass for negatively charged particles produced in ${ }^{28} \mathrm{Si}+\mathrm{Au}$ and ${ }^{28} \mathrm{Si}+\mathrm{Cu}$ interactions at $14.6 \mathrm{~A} \mathrm{GeV} / \mathrm{c}$ in the rapidity interval $2.2<y<2.6$ [Lov91]. They pointed out a substantial deviation from exponential at low $m_{T}$ compared to the $p p$ data of Blobel et al. at 12 and $24 \mathrm{GeV} / c$ which was exponential in $m_{T}$ down to $p_{T}=0$ [Blo74]. After arguing that contributions from misidentified electrons and $K^{-}$were likely to be negligible, they asserted that the effect represented an excess production of low $p_{T}$ pions in nucleus-nucleus collisions. However, their data were preliminary, corrected only for acceptance, and they did not attempt to quantify the result by constructing the ratio with respect to the $p p$ data as a function of $p_{T}$. We emphasize that the most dramatic examples of low- $p_{T}$ enhancement in $A A$ collisions have been observed for asymmetric systems and near target rapidity. A similar effect has been observed in $h A$ collisions by two Fermilab experiments [Gar77], [Cha79]. They were able to parametrize the invariant cross section in $h A$ with a $p_{T}$-dependent power of the target mass $A$

$$
\sigma_{p A}^{i n v}\left(p_{T}\right)=\sigma_{p p}^{i n v}\left(p_{T}\right) A^{\alpha\left(p_{T}\right)} .
$$

This parametrization was employed by Cronin et al. to describe the anomalous nuclear enhancement at high $p_{T}$ [Cro75]. For charged pions, the power function $\alpha\left(p_{T}\right)$ passed through a minimum at $p_{T} \sim 0.400 \mathrm{GeV} / c$.

Like experiment NA34, we will compare our data to the ISR data for $\sqrt{s}=23$ $\mathrm{GeV}$. Alper et al. [Alp75] measured the production spectra of charged hadrons at fixed rapidities at center-of-mass energies from $\sqrt{s}=23$ to $63 \mathrm{GeV}$ and obtained a good representation of the pion data for $p_{T} \lesssim 1.2 \mathrm{GeV} / c$ by including a quadratic term in $p_{T}$,

$$
E \frac{d^{3} \sigma}{d p^{3}}=A \exp \left(-b p_{T}+c p_{T}^{2}\right) \exp \left(-d y^{2}\right) .
$$

Over their full acceptance up to $p_{T} \sim 5 \mathrm{GeV} / c$, they used a parametrization with a high $p_{T}$ behavior suggested by the parton model of Blankenbecler and Brodsky [Bla74]

$$
E \frac{d^{3} \sigma}{d p^{3}}=A_{1} \exp \left(-b p_{T}\right) \exp \left(-d y^{2}\right)+A_{2} \frac{\left(1-p_{T} / p_{\text {beam }}\right)^{m}}{\left(p_{T}^{2}+c^{2}\right)^{n}} .
$$

The parameters $m$ and $n$ were predicted from simple quark-counting rules. Because their cross section measurements are tabulated in Ref. Alp75, we could compare their fit results to their data. Neither Equation 6.21 nor Equation 6.22 provides a good fit to the data at $\sqrt{s}=23 \mathrm{GeV}$ and $y=0$ with the parameters tabulated in Ref. Alp75. We refit the $\sqrt{s}=23, y=0$ data for $0<p_{T}<1.9$ to Equation 6.21 both with $A, b$, and $c$ all free to vary and with $b$ fixed to the published value $b=6.8$. We obtained $A=178 \pm 10, b=7.20 \pm 0.14, c=0.855 \pm .065$ and $A=153 \pm 3$, $c=0.675 \pm .014$, respectively. We fit our measured data to Equation 6.21 with all parameters free and obtained $A=507 \pm 26, b=6.10 \pm 0.26$ and $c=0.583 \pm 0.23$ 
for our midrapidity data set $2.0<y<2.8$. Within statistical errors the same values for $b$ and $c$ were obtained for $1.0<y<2.0$.

The results of the fits are shown in the upper panel of Figure 6.7. The solid line shows the fit of Equation 6.21 to our data, the dashed and dotted lines the fits to the data of Ref. Alp75 with the slope parameter $b$ free to vary and fixed at 6.8 , respectively. The fits to the $p p$ data have been renormalized to have the same value as the $A A$ data at $p_{T}=0$. Note that because $b$ is smaller for our data than for either fit to the $p p$ data, and because $c$ is not large enough to compensate for $p_{T}<2.0 \mathrm{GeV} / c$, our spectra always decrease less rapidly with $p_{T}$ than the $p p$ invariant cross section-instead of a low-p $p_{T}$ enhancement there is a steady rise in the relative yield with $p_{T}$. This is shown in the lower panel of Figure 6.7 where the ratio of our fit to the $p p$ fit is shown for the two cases, the solid line for the free parameter fit, the dashed line for the fit with $b$ fixed. The shape of the ratio plot is qualitatively similar to one made by the Axial Field Spectrometer collaboration comparing the normalized invariant cross sections of charged particles from $\alpha \alpha$ and $p p$ collisions at $\sqrt{s}=31 \mathrm{GeV} /$ nucleon st the ISR [Ake82]. They employed a minimum bias trigger to determine the ratio for $0.250 \lesssim p_{T}<2.0$ and a high-p single particle trigger for $p_{T}>2.0 \mathrm{GeV} / c$. Their ratio plot increases monotonically up to $p_{T} \sim 3.0 \mathrm{GeV} / c$.

The behavior of the cross section ratios for symmetric $A A$ collisions suggests that the low- $p_{T}$ enhancement observed in central collisions of unequal mass nuclei is a spectator matter effect. Garbut et al. [Gar77] suggested that the turnover in the power function $\alpha\left(p_{T}\right)$ at low $p_{T}$ in $h A$ collisions was related to variations in the center-of-mass kinematics for measurements made at fixed laboratory angles. Low $p_{T}$ particles at fixed laboratory angles are closer in rapidity to the nuclear targetthe turnover in $\alpha\left(p_{T}\right)$ at low $p_{T}$, and similarly the low-p $p_{T}$ enhancement in $A A$, could be associated with target fragmentation from spectator matter. Another possible spectator effect would be particle production from the cascading of participant final-state particles through cold spectator matter. We have already concluded from the average particle production per participant $N N$ pair that cascading is a weak effect in central ${ }^{32} \mathrm{~S}+{ }^{32} \mathrm{~S}$ interactions at $200 \mathrm{GeV} /$ nucleon. Both these spectator mechanisms would explain why the low- $p_{T}$ enhancement is stronger near target rapidity and for asymmetric systems. The preliminary observation of a lowpr enhancement by Love et al. [Lov91] for particles emitted in the forward rapidity hemisphere in the center-of-mass is something of a puzzle if longitudinal growth applies at AGS energy.

The best fits to the negative hadron $p_{T}$ spectra are obtained from the twocomponent forms of Equation 6.14, 6.13, 6.19, and 6.15. The results of leastsquares fits in the two rapidity intervals into which we have subdivided our data are tabulated in Table 6.2. $A_{1}, T_{1}$ and $A_{2}, T_{2}$ are the normalization contributions and temperature parameters of the low and high temperature components, respectively. The low-temperature component dominates at low $p_{T}$ and has the steeper asymptotic slope. As evidenced by the equality of the $\chi^{2}$ NDF all fits equally well describe the data. Although the different expressions introduce small but discernible differences for $p_{T}>1.0 \mathrm{GeV} / c$, the data cannot discriminate amongst 


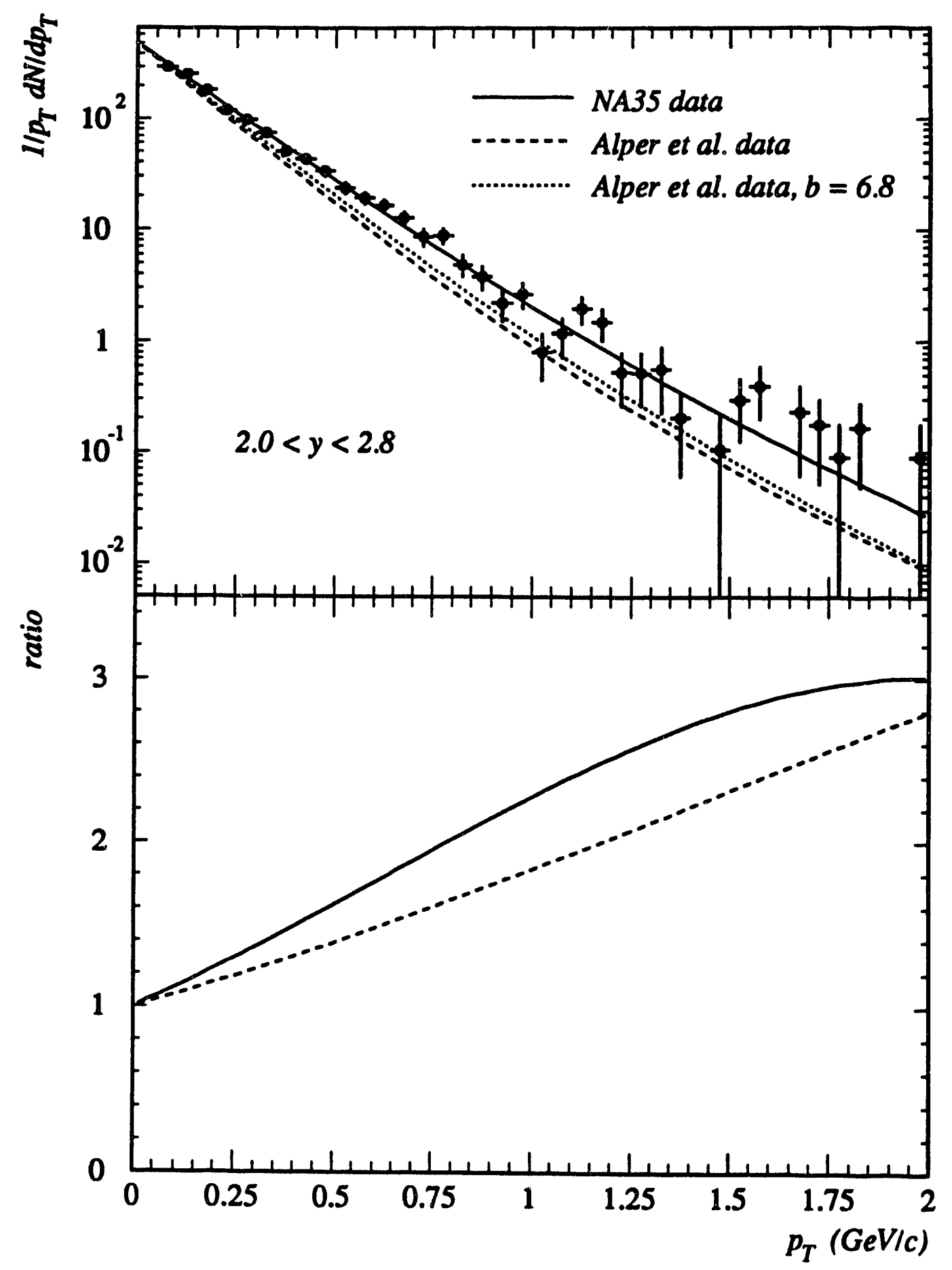

Figure 6.7: Linear plus quadratic exponential fit to negative hadron $p_{T}$ distributions. The solid line shows the fit of Equation 6.21 to our data, the dashed and dotted lines the fits to the data of Ref. Alp75 with the slope parameter $b$ free to vary and fixed at 6.8 , respectively. The fits to the $p p$ data have been renormalized to have the same value as the $A A$ data at $p_{T}=0$. The lower panel shows the ratios of the fit to the $A A$ data to the two fits to the $p p$ data. 


\begin{tabular}{|c|c|c|c|c|c|c|}
\hline$f\left(p_{T}\right)$ & $A_{1}$ & $T_{1}(\mathrm{MeV})$ & $\overline{A_{2}}$ & $T_{2}(\mathrm{MeV})$ & $R(\%)$ & $\chi^{2} / \mathrm{NDF}$ \\
\hline \multicolumn{7}{|c|}{$1.0<y<2.0$} \\
\hline \multicolumn{7}{|c|}{$<p_{T}>=0.359 \pm .006 \mathrm{GeV} / c$} \\
\hline 1 & $8700 \pm 2500$ & $74.6 \pm 9.0$ & $830 \pm 210$ & $178 \pm 8$ & $68 \pm 8$ & $25.1 / 26$ \\
\hline 2 & $940 \pm 250$ & $82 \pm 14$ & $283 \pm 61$ & $195 \pm 9$ & $75 \pm 8$ & $25.7 / 26$ \\
\hline 3 & $14600 \pm 2600$ & $64.7 \pm 4.7$ & $940 \pm 200$ & $159 \pm 6$ & $59 \pm 5$ & $24.5 / 26$ \\
\hline 4 & $3920 \pm 820$ & $72.6 \pm 7.6$ & $510 \pm 110$ & $176 \pm 8$ & $65 \pm 7$ & $24.9 / 26$ \\
\hline \multicolumn{7}{|c|}{$2.0<y<2.8$} \\
\hline \multicolumn{7}{|c|}{$<p_{T}>=0.365 \pm .006 \mathrm{GeV} / c$} \\
\hline 1 & $14700 \pm 7200$ & $63 \pm 11$ & $1360 \pm 300$ & $171 \pm 7$ & $78 \pm 7$ & $29.8 / 29$ \\
\hline 2 & $1440 \pm 730$ & $66 \pm 16$ & $430 \pm 74$ & $189 \pm 8$ & $84 \pm 7$ & $29.6 / 29$ \\
\hline 3 & $22700 \pm 6300$ & $57.1 \pm 5.8$ & $1620 \pm 320$ & $151 \pm 6$ & $68 \pm 6$ & $30.2 / 29$ \\
\hline 4 & $5990 \pm 2060$ & $62.4 \pm 9.3$ & $820 \pm 160$ & $168 \pm 7$ & $75 \pm 8$ & $29.7 / 29$ \\
\hline
\end{tabular}

Table 6.2: Summary of two-component fits to the negative hadron $1 / p_{T} d N / d p_{T}$ distributions: 1) Equation 6.14, 2) Equation 6.13, 3) Equation 6.19, 4) Equation 6.15. The low-temperature component dominates at low $p_{T}$ and has the steeper asymptotic slope. The relative yield in the high-temperature component $R$ is computed by weighting the fitted expressions by $p_{T}$ and integrating from $p_{T}=0.0$ to $p_{T}=2.0 \mathrm{GeV} / c$. 
them on the basis of goodness-of-fit. The statistical errors on the fitted parameters $A_{1}, T_{1}, A_{2}$, and $T_{2}$ correspond to the changes in the respective parameter which would produce a unit change in the weighted $\chi^{2}$ function with the other parameters held fixed. The errors were checked by employing the same Monte Carlo procedure used to assign errors to the extrapolated points and integrated yield of the $d N / d y$ distributions. To obtain the mean transverse momentum $\left\langle p_{T}\right\rangle$ and the relative yield in the high-temperature component $R$ we weighted the fitted expressions by $p_{T}$ and integrated from $p_{T}=0$ to $p_{T}=2.0 \mathrm{GeV} / c$, for example

$$
R=\frac{\int p_{T} f_{2}\left(p_{T}, A_{2}, T_{2}\right) d p_{T}}{\int p_{T}\left\{f_{1}\left(p_{T}, A_{1}, T_{1}\right)+f_{2}\left(p_{T}, A_{2}, T_{2}\right)\right\} d p_{T}} .
$$

The errors in the normalization coefficients $A_{1}$ and $A_{2}$ are large, especially $A_{1}$ which depends only on a small number of data points below $p_{T} \sim 0.400 \mathrm{GeV} / c$. In spite of this, the errors on $T_{1}, T_{2}, R$ and $\left\langle p_{T}\right\rangle$, defined as the rms deviations of the fit parameters over the Monte Carlo ensemble, are relatively small. The mean transverse momentum is the same for all the two-component fits.

As an example, the fits to the two-component form of Equation 6.14 are plotted as the solid lines in Figure 6.8. The dotted and dashed lines indicate the relative contributions of the low and high temperature components. If $R$ is mistakenly computed from the relative areas under the curves rather than the $p_{T}$-weighted integrated values, the yield in the high-temperature component will be underestimated by $\sim 20 \%$. We have chosen this particular expression because it occurs naturally in thermal models which include one-dimensional, longitudinal expansion. Pugh has pointed out that both Equation 6.14 and its high temperature limit, Equation 6.15, approximate expressions for small rapidity intervals which introduce longitudinal dynamics [Pug90]. An explicit example of this can be found in Kataja and Ruuskanen [Kat90] who obtain the following expression for the invariant cross section at $y=y_{0}$ for the case of a thermal distribution expanding longitudinally with no transverse flow,

$$
\left.\frac{1}{p_{T}} \frac{d^{2} N}{d y d p_{T}}\right|_{y=\nu_{0}} \propto m_{T} \times \sum_{n=1}^{\infty}( \pm 1)^{n+1} \exp (n \mu / T) K_{1}\left(n m_{T} / T\right) .
$$

In the Maxwell-Boltzmann limit only $n=1$ contributes and this reduces to Equation 6.14. Near midrapidity, one-dimensional hydrodynamic flow leads to the same $p_{T}$ distribution as the rapidity-integrated thermal distribution without flow. Because of the physical motivation provided by the negative hadron and proton rapidity distributions-that they can be simply understood if we assume nuclear transparency, thermal equilibrium and longitudinal, hydrodynamic expansion until freezeout-we single out Equation 6.14 as the one most likely to return a fit parameter $T$ which can be interpreted as a physical temperature.

Within statistical errors, the mean transverse momentum and temperature parameters are independent of rapidity for $y>1.0$, quantitative evidence that the invariant cross section approximately factorizes. The same conclusion was reached by Kafka et al. [Kaf77] for the $\left\langle p_{T}\right\rangle$ of $\pi^{+}$and $\pi^{-}$produced in $p p$ collisions 


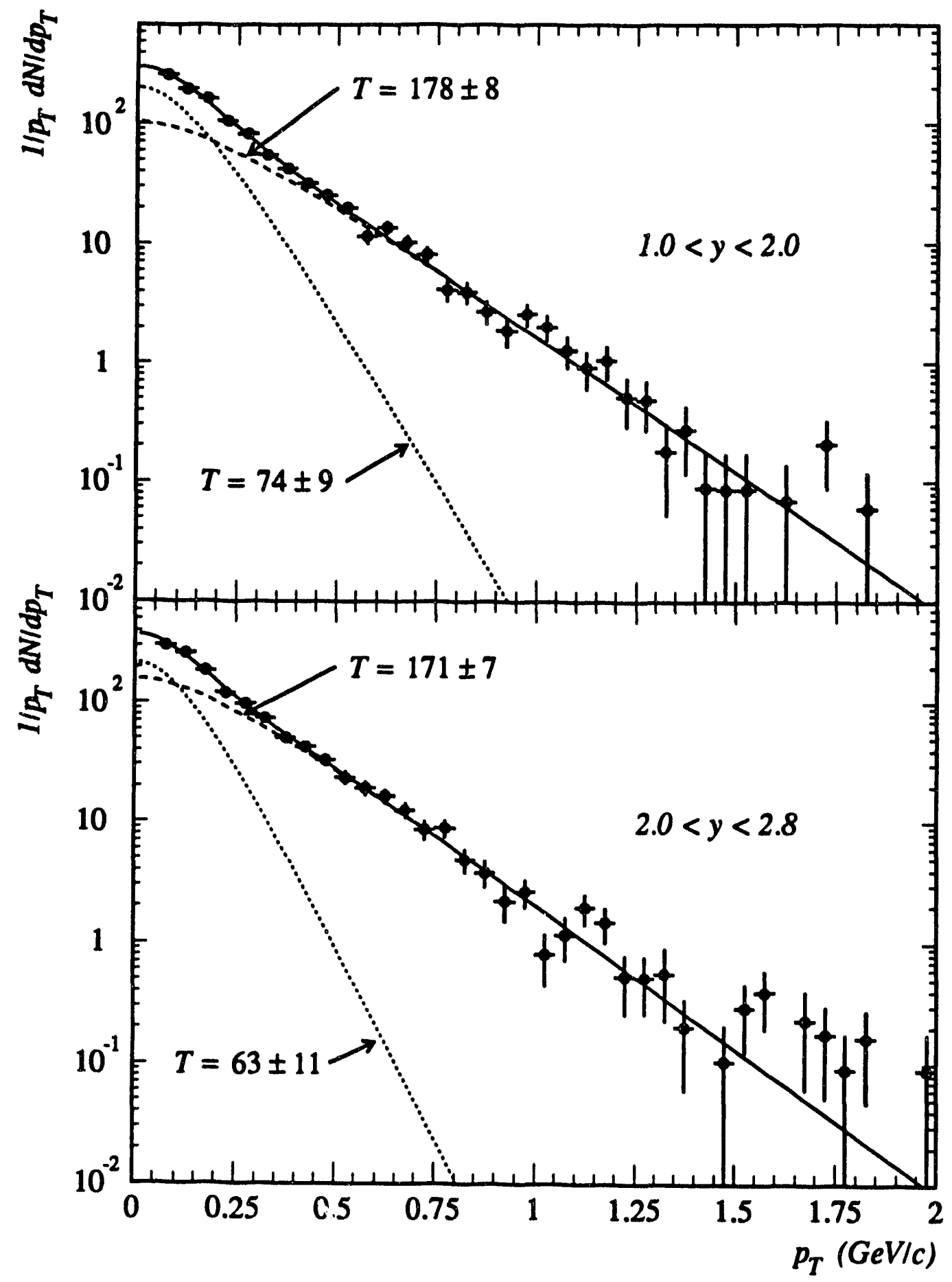

Figure 6.8: Two-component thermal fits to negative hadron $p_{T}$ distributions. Two different rapidity intervals are shown. The solid lines plot the results for the twocomponent form of Equation 6.14. The dashed and dotted lines show the respective contributions of the high and low temperature components. 
at $205 \mathrm{GeV} / c$. The mean $p_{T}$ we have measured, $\left\langle p_{T}\right\rangle=0.359 \pm .006$ and $<p_{T}>=0.365 \pm .006 \mathrm{GeV} / c$ for $1.0<y<2.0$ and $2.0<y<2.8$ respectively, is comparable to that obtained in $p p$ collisions at comparable energies. For $y>1.0$, Kafka et al. reported $\left\langle p_{T}\right\rangle \approx 0.350 \mathrm{GeV} / c$. From our fit to the midrapidity $p p$ data of Alper et al. at $\sqrt{s}=23 \mathrm{GeV}$, extrapolated to $p_{T}=0$, we obtained $\left\langle p_{T}\right\rangle \approx 0.325 \mathrm{GeV} / c^{10}$ Like our data, the bubble chamber data of Kafka et al. are really negative hadron data, with rapidity calculated assuming the pion mass. At fixed $\sqrt{s}$, a systematic increase in $\left\langle p_{T}\right\rangle$ with increasing projectile and target mass for central collisions of equal mass nuclei, combined with the monotonically increasing cross section ratio as a function of $p_{T}$ shown in Figure 6.7, would provide evidence for rescattering of produced particles in participant nuclear matter. A small effect of this kind has been observed for $\alpha \alpha$ compared to $p p$ collisions at the ISR at $\sqrt{s}=31 \mathrm{GeV}$ [Fae84].

It is clear from Table 6.2 that both the temperature parameters and the relative yield $R$ depend strongly on the functional form fitted to the data. Note also the large discrepancy between $T_{1}$ and $T_{2}$ and the temperature $T_{B}$ deduced from our single-component Boltzmann fits over a restricted $p_{T}$ acceptance, $T_{B} \approx 140 \mathrm{MeV}$. The same discrepancy occurs at AGS energy. Stachel reported [Sta91] that the preliminary data from experiment $E 810$, which is able to measure down to $p_{T}=0$ and has also obtained a data point for $p_{T} \sim 1.3 \mathrm{GeV} / c$, can be described by a two-component exponential in transverse mass with $T_{1}=83$ and $T_{2}=188 \mathrm{MeV}$ and $R=72 \%$. This is to be compared to the E802 result for $0.3<p_{T}<1.0$, $T_{B} \approx 125 \mathrm{MeV}$. Brown, Stachel and Welke [Bro91] approximately reproduced the E810 $p_{T}$ spectrum by a Monte Carlo simulation which assumed chemical and thermal equilibrium for a hadron gas consisting of pions, nucleons, and $\Delta$ resonances at $T=150 \mathrm{MeV}$ and which employed the Landau fireball expression Equation 6.3 to populate the single-particle inclusive distributions. Pions from resonance decay are responsible for the two-component spectral shape. Because they underestimated the measured cross section for $p_{T} \gtrsim 0.750 \mathrm{GeV} / c$, they concluded that the underlying temperature in $14.6 \mathrm{GeV} /$ nucleon $\mathrm{Si}+\mathrm{Au}$ central collisions exceeded $150 \mathrm{MeV}$. Moreover, they obtained an apparent temperature $T_{\text {app }}=125 \mathrm{MeV}$ by fitting their Monte Carlo spectrum to a single exponential in $m_{T}$ over the E802 acceptance. Interestingly, the fits of our data to the two-component exponential in $m_{T}$, the second entries in Table 6.2, obtain similar values for $T_{1}, T_{2}$, and $R$ as the E810 results reported by Stachel. For example, for $1.0<y<2.0$ we found $T_{1}=82 \pm 14, T_{2}=195 \pm 9$, and $R=75 \pm 8$.

The fully corrected proton $p_{T}$ spectrum $1 / p_{T} d N / d p_{T}$ as a function of $p_{T}$ is shown in Figure 6.9 for the rapidity interval $0.6<y<1.4$, over which our $p_{T}$ acceptance is unbiased. The non-invariant spectrum for the same data $d N / d p_{T}$ were plotted on a linear scale in Figure 5.24. The solid line is a single-component thermal fit to Equation 6.14 which yields $T=168 \pm 10 \mathrm{MeV}$. Note that the proton

\footnotetext{
${ }^{10}$ The bubble chamber data of Kafka et al. were measured down to $p_{T}=0$ and they explicitly plotted $\left\langle p_{T}\right\rangle$ as a function of rapidity $y$. The spectrometer data of Alper et al. were measured down to $p_{T} \sim .200 \mathrm{GeV} / c$. Our fit to their data assumes the cross section turns over as $p_{T} \rightarrow 0$, rather than rises exponentially.
} 


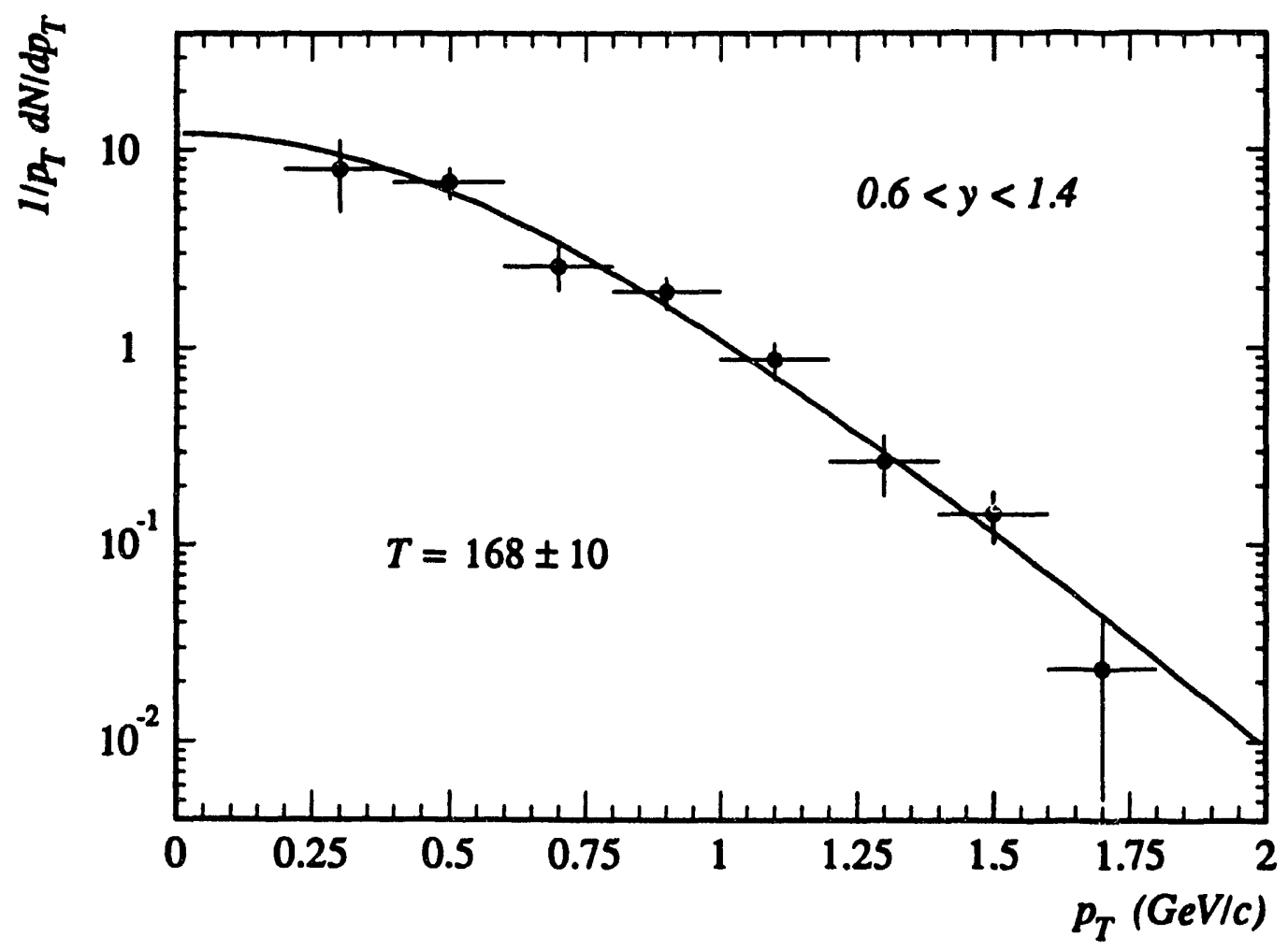

Figure 6.9: Proton transverse momentum distribution. The solid curve is the fit to Equation 6.14. The $p_{T}$ acceptance is unbiased in the rapidity interval shown.

\begin{tabular}{||cccc||}
\hline \hline$f\left(p_{T}\right)$ & $A$ & $T(\mathrm{MeV})$ & $\chi^{2} / \mathrm{NDF}$ \\
\hline \multicolumn{4}{|c||}{$0.6<y<1.4$} \\
\multicolumn{4}{|c}{$<p_{T}>=0.595 \pm .026 \mathrm{GeV} / c$} \\
1 & $6100 \pm 2900$ & $168 \pm 10$ & $5.2 / 6$ \\
2 & $2400 \pm 1100$ & $178 \pm 11$ & $5.3 / 6$ \\
3 & $4800 \pm 2200$ & $158 \pm 8$ & $5.0 / 6$ \\
4 & $3400 \pm 1500$ & $167 \pm 9$ & $5.2 / 6$ \\
& & & \\
\hline
\end{tabular}

Table 6.3: Summary of single-component firs to the proton $1 / p_{T} d N / d p_{T}$ distribution. The functional forms are the same as in Table 6.2. 
$p_{T}$ spectrum can be well described without invoking a second component. The results of the different thermal model fits are summarized in Table 6.3. If we omit the non-vertex particle correction, which has a strong effect on the shape of the spectrum for $p_{T} \gtrsim 1.2 \mathrm{GeV} / c$ (see Figure 5.22), the temperature parameters of all fits are systematically higher by $\sim 8 \mathrm{MeV}$ and the mean $p_{T}$ increases to $\sim 0.615$ $\mathrm{GeV} / c$. The mean $p_{T}$ we observe, $\left\langle p_{T}\right\rangle=0.595 \pm .026 \mathrm{GeV} / c$, is dramatically higher than that observed in $p p$ collisions at the same $\sqrt{s}$. Using the parametrization of Equation 6.22 and the fit parameters obtained by Alper et al. to determine the mean $p_{T}$ of midrapidity protons at $\sqrt{s}=23 \mathrm{GeV}$ for $0.0<p_{T}<2.0 \mathrm{GeV} / c$, we calculate $\left\langle p_{T}\right\rangle=0.415 \mathrm{GeV} / c$. This large increase in central ${ }^{32} \mathrm{~S}+{ }^{32} \mathrm{~S}$ collisions compared to $p p$ is strong evidence for nucleon rescattering in participant matter which may be an important mechanism driving nuclear matter toward thermal equilibrium. Combined with our estimates in Section 6.1 of the mean baryon rapidity shift and the mean number of negative hadrons per participant $N N$ pair, which argued for an absence of intranuclear cascading during the interpenetration phase of the collision, the high proton $\left\langle p_{T}\right\rangle$ indicates that rescattering in participant matter occurs during the expansion phase. This effect is unique to central nucleus-nucleus collisions and suggests that the nuclear fragmentation regions will remain important places to look for new physics as the available energy in heavy-ion accelerators continues to increase.

Two important features of the transverse momentum distributions are evident from comparison of the negative hadron and proton spectra:

1. The proton spectrum can be described by a simple thermal model while the negative hadron spectra require a superposition of two thermal sources at different temperatures to describe their shape.

2. Within errors, the proton distribution has the same temperature as the hightemperature component of the negative hadron distribution.

We will consider two models which have attempted to account for the concave shape of the negative pion $p_{T}$ spectrum, to account for the different shape of the proton $p_{T}$ spectrum, and a.t the same time reconcile the apparently different temperatures deduced from single-component fits over limited $p_{T}$ acceptances, which undermine a coherent interpretation of the data within a thermal equilibrium picture. One model, the collective flow model of Lee, Heinz and Schnedermann [Lee89], [Lee90], extended the blast wave model of Siemens and Rasmussen [Sie79] by superimposing an isentropic radial expansion with a radially increasing expansion velocity on a hadronic resonance gas in thermal and chemical equilibrium. The influence of a common collective flow velocity ocurs over different regions of momentum for hadrons of different mass, leading to different spectral shapes. Although Lee, Heinz and Schnedermann were able to obtain a concave pion $p_{T}$ spectrum and a proton p1 spectrum with a high apparent temperature, both of which fit film data from the NA35 streamer chamber [Lee90], their spherically expanding fireball picture failed badly at describing the longitudinal degree of freedom. Their flow model predicted a rapidity distribution even narrower than the thermal one obtained from 
Equation 61 at $T=200 \mathrm{MeV}$, much narrower than the data. Moreover, as noted by Jacak lac91a], the flow velocity is very sensitive to the curvature in the pion spectr:-i-if the concavity at low $p_{T}$ can be accounted for by different processes the extracted velocity will be much smaller. Brown, Stachel and Welke [Bro91] pointed out that the flow should be predominantly longitudinal since the initial pressure gradients are much greater in the longitudinal direction and suggested that Lee et al. strongly overestimate the transverse fow. For these reasons we consider a second model, which explicitly examines the impact of resonance decay kinematics on the pion and proton $p_{T}$ spectra, a more realistic and natural choice-it invokes well-understood processes from $p p$ physics which must occur in the nucleus-nucleus data.

The resonance idea is not new. Hagedorn and Ranft [Hag68] singled out the powerful distorting influence two-body resonance decays could exert on thermal particle spectra. Brockmann et al. [Bro84] showed that the low apparent temperature of pions in central $\mathrm{Ar}+\mathrm{KCl}$ collisions at $1.8 \mathrm{GeV} /$ nucleon at the BEVALAC could be explained by the decay kinematics of $\Delta$ resonances in thermal equilibrium with nucleons at the same temperature. We have already referred to the application of the same approach to the AGS pion spectra by Brown, Stachel and Welke [Bro91]. Sollfrank, Koch and Heinz [Sol90] and Barz, Bertsch, Kusnezov and Schulz [Bar91] were the first to assert that the production of higher resonances is an important factor in high-energy nucleus-nucleus collisions and explicitly calculate the contributions of their decays to the final-state $p_{T}$ spectra measured by NA35 at CERN. Although Barz et al. were unable to reproduce the negative hadron spectra for $p_{T}<0.2 \mathrm{GeV} / c$, Solifrank et al. found excellent agreement between the $p_{T}$ spectra predicted by their model and the NA35 data for $\pi^{-}, p, K_{S}^{0}$, and $\Lambda$. Both calculations assumed a thermodynamic model with a single temperature $T$ and determined the relative hadronic abundances by assuming chemical equilibrium. ${ }^{11}$

The model of Sollfrank et al. assumes an ideal relativistic hadron gas with constant temperature $T$ and no collective motion. Stable hadrons and resonances are assumed to be in thermal and chemical equilibrium, each at a chemical potential $\mu=b \mu_{\mathrm{b}}+s \mu_{\mathrm{s}}$ where $b$ and $s$ are the baryon number and strangeness of the hadron or resonance and $\mu_{\mathrm{b}}$ and $\mu_{\mathrm{s}}$ are chemical potentials which constrain the net baryon number and strangeness of the system. Because of strangeress conservation, $\mu_{\mathrm{s}}=f\left(\mu_{\mathrm{b}}, T\right)$ leaving $T$ and $\mu_{\mathrm{b}}$ as free parameters. Equation 6.17 is integrated over all rapidities to determine particle transverse momenta. Note that for all particles more massive than the pion (and for pions with $p_{T} \gtrsim 0.3$ $\mathrm{GeV} / \mathrm{c}$ ), the Maxwell-Boltzmann limit may be used and the transverse momentum distributions are determined from

$$
\frac{1}{p_{T}} \frac{d N}{d p_{T}}=\frac{g}{4 \pi^{2}} \exp (\mu / T) m_{T} K_{1}\left(m_{T} / T\right),
$$

\footnotetext{
${ }^{11}$ It is unclear why these similar calculations obtained such different results. The main differences in the models are the inclusion by Sollfrank et al. of a number of resonances omitted by Barz et al. and the use of a single thermal fireball compared to a superposition of fireballs uniformly spread in rapidity along the beam axis.
} 
the form we have used to extract the physical temperatures from the measured data and suggested by longitudinal expansion. Here $g$ is the statistical degeneracy and $\mu$ is determined for each stable particle or resonance from $\mu=b \mu_{\mathrm{b}}+s f\left(\mu_{\mathrm{b}}, T\right)$. It is clear from Equation 6.25 that once $T$ and $\mu_{\mathrm{b}}$ are specified, the momentum distribution and relative abundance of each hadron and resonance are fixed by the assumption of chemical equilibrium. There is no evolution in the model-the fireball disassembles instantaneously, without final-state interactions, and resonances decay into lower mass, stable particles. The contribution of a decay channel to a particular final state is calculated by properly folding the decay kinematics onto the momenturn distribution of the parent resonance.

The following decay channels were included in the calculation:

$$
\begin{aligned}
\eta & \rightarrow \pi^{-} \pi^{+} \pi^{0} \\
\rho^{0,-} & \rightarrow \pi^{-} \pi^{+, 0} \\
\omega & \rightarrow \pi^{-} \pi^{+} \pi^{0} \\
K^{*}-, 0 & \rightarrow \pi^{-} K^{0,+} \\
\Delta[\bar{\Delta}] & \rightarrow \pi^{-} N[\bar{N}] \\
\Sigma^{*}\left[\bar{\Sigma}^{*}(1385)\right] & \rightarrow \pi^{-} Y[\bar{Y}] .
\end{aligned}
$$

Only the $\Delta$ contributes to the proton spectrum. Note that only the $\rho$ undergoes a two-body, mass-symmetric decay. This is important. In a two-body decay to daughter particles with widely different masses, the mass-dependence of the Lorentz boost from the resonance rest frame to the laboratory frame leads to a strong asymmetry in the observed momentum. In the laboratory, the heavy particle typically has most of the momentum, resulting in a concentration of pions from twobody, mass-asymmetric decays at low $p_{T}$. At the temperature and baryon chemical potentials considered in Ref. Sol $90, T=200 \mathrm{MeV}$ and $\mu_{\mathrm{b}}=100-200 \mathrm{MeV}$, only $\pi^{-}$from $\rho$ decay have enough momentum to significantly populate the $p_{T}$ distribution for $p_{T} \gtrsim 0.600 \mathrm{GeV} / c$. Generally, pions from two-body decay exhibit a constant, asymptotic slope for $p_{T} \gtrsim m_{\pi}$ which is steeper than that of the parent resonance. The pions from thres-body decay have a nearly constant slope which is much steeper than their parent resonance. Sollfrank et al. pointed out that, because of the constant asymptotic slopes, effective temperatures $T_{\text {eff }}<T_{\text {thermal }}$ could be assigned to pions from resonance decay. The two-component, thermal parametrization embodies the significance of the underlying resonance structure to the negative hadron spectra. The low-temperature component is the superposition of thermal pions and pions from the decay of open resonance channels at a given energy scale. The high-temperature component is the superposition of thermal pions and pions from the open resonance channels which undergo twobody, mass-symmetric decay. Fits to the high-temperature component should give reasonable estimates of the thermal temperature.

For $T=200 \mathrm{MeV}$ and $\mu_{\mathrm{b}}=200 \mathrm{MeV}$, Sollfrank et al. tabulated the relative contributions of thermal pions and pions from the different decay channels. The yield of thermal pions and pions from $\rho$ decay sum to $\approx 60 \%$, in semi-quantitative agreement with the values we have calculated for the relative yield in the second 
component, $R=68 \pm 8$ and $78 \pm 7$, near target fragmentation and near midrapidity, respectively. Thermal pions only account for $30 \%$ of the total. Barz et al. [Bar91] also calculated the resonance yields for a model suggested by a string-breaking picture, with statistical weights determined solely from spin and isospin degeneracy, and found that the number of pions from $\rho$ decay exceeded the number of direct pions. In the thermal model the relative yields of direct and resonance pions depend strongly on temperature. At $T=150 \mathrm{MeV}$, Sollfrank et al. noted that the contribution from decay pions dropped to only $40 \%$ [Sol90]. Nevertheless, the agreement between the model calculation and the data is rather striking

The proton spectrum is simple to understand because only one resonance, the $\Delta$, is likely to be important [Bro91]. Because the proton is the massive finalstate particle in $\Delta$ decay, resonance decay protons contribute to the transverse momentum spectra over the same $p_{T}$ scale as do the thermal protons. Although the asymptotic slope of resonance decay protons is somewhat steeper than that of the thermal protons, the proton spectral shape is relatively insensitive to the effect of resonance decay. The temperature extracted from a single-component thermal fit to the proton spectrum will provide a good estimate of the underlying physical temperature. This accounts for the agreement between the proton mperature and the high temperature components of the negative hadron spectra. Based on the resonance picture and our fits to the data we conclude that the charged particle $p_{T}$ spectra are indeed thermal with an estimated temperature

$$
T_{f} \sim 170 \mathrm{MeV}
$$

at freezeout. The agreement between the proton and negative hadron temperatures confirms the assumption that the expansion is mainly longitudinal and has a negligible effect on the transverse momentum distributions. Because the pions from $\rho$ decay and the protons from $\Delta$ decay have steeper slopes than their thermal counterparts, which can only increase the slopes of the detectable final-state distributions, $T_{f}$ may be somewhat higher than the fitted value.

We are now in a position to estimate the baryon density per $\mathrm{fm}^{3}$ and the baryon chemical potential. Although we have emphasized the symmetric Landau two-fireball model, the negative hadron $d N / d y$ distribution is consistent with a plateau of \pm 1 unit of rapidity about midrapidity. The proton $d N / d y$ distribution is also fairly tlat. Thus the condition for the validity of the scaling hydrodynamic solution is approximately satisfied near midrapidity. Recall from Equation 1.37,

$$
n_{\mathrm{b}}\left(\tau_{0}\right)=\frac{1}{\tau_{0} A_{T}} \frac{d N_{\mathrm{b}}}{d y} .
$$

The cross-sectional overlap area of two circles of equal radii can be employed to calculate $A_{T}$ as a function of impact parameter $b$,

$$
A_{T}(b)=2 R^{2}\left\{\cos ^{-1}\left(\frac{b}{2 R}\right)-\left(\frac{b}{2 R}\right)\left[1-\left(\frac{b}{2 R}\right)^{2}\right]^{1 / 2}\right\}
$$


For $b<R, A_{T}$ is approximately linear in $b$ which implies that

$$
<A_{T}(b)>=A_{T}(<b>) \text {. }
$$

Because our Monte Carlo has a good absolute normalization with respect to our data, we can estimate

$$
<b>=2 / 3 b_{\max }=2 / 3(2.5 \mathrm{fm})=5 / 3 \mathrm{fm}
$$

which for $R \sim 3.5 \mathrm{fm}$ yields

$$
A_{T} \sim 25 \mathrm{fm}^{2} .
$$

Using the canonical value $\tau_{0}=1 \mathrm{fm} / c$, Equation 6.26 gives

$$
n_{b}\left(\tau_{0}\right)=\frac{2(2.3)}{1(25)}=0.18 \text { baryons per } \mathrm{fm}^{3}
$$

where the factor of two in the numerator converts proton rapidity density to baryon rapidity density for the isoscalar ${ }^{32} \mathrm{~S}+{ }^{32} \mathrm{~S}$ system. If the expansion is very rapid, so that we can approximate $T\left(\tau_{0}\right)$ by $T_{f}$, then Equation 1.21 for the net baryon number density of a two-flavor plasma as a function of $\mu_{\mathrm{q}}$ and $T$, under the assumptions outlined in Section 1.2.3, yields an upper bound

$$
\mu_{\mathrm{q}}=71 \mathrm{MeV} \quad \Longrightarrow \quad \mu_{\mathrm{b}}=210 \mathrm{MeV}
$$

Bjorken argued [Bjo83] that under general conditions

$$
c^{2} \leq 1 / 3
$$

so that from Equation 1.40 the temperature decreases as $\tau^{-1 / 3}$ or slower. Since estimates for the duration of the longitudinal expansion [Kaj83b] are typically $A^{1 / 3} \sim 5 \mathrm{fm} / c$ for large nuclei, we obtain a lower bound for the chemical potentials in ${ }^{32} \mathrm{~S}+{ }^{32} \mathrm{~S}$ at $200 \mathrm{GeV} /$ nucleon by calculating $\mu_{\mathrm{q}}$ for an initial temperature

$$
T\left(\tau_{0}\right)=T_{f}\left(\frac{\tau}{\tau_{0}}\right)^{1 / 3} \sim T_{f}(5)^{1 / 3} \sim 290 \mathrm{MeV} .
$$

The result,

$$
\mu_{\mathrm{q}}=25 \mathrm{MeV} \quad \Longrightarrow \quad \mu_{\mathrm{b}}=75 \mathrm{MeV} \text {. }
$$

In the same spirit we estimate the energy density from the Bjorken formula

$$
\varepsilon_{0}=\frac{<m_{T}>d N}{\tau_{0} A_{T}} \frac{d N}{d y} .
$$

From the transverse momentum distributions, the mean $m_{T}$ for negative hadrons and protons is 0.390 and $1.11 \mathrm{GeV} / \mathrm{e}$, respectively, which leads to the energy density in pions and nucleons

$$
\varepsilon \sim 3 \varepsilon_{\pi}+2 \varepsilon_{p}=\frac{1}{1(25)}(3 \times 20 \times 0.390+2 \times 2.3 \times 1.11),
$$




$$
\sim 1.1 \frac{\mathrm{GeV}}{\mathrm{fm}^{3}} \text {. }
$$

From the NA35 film measurement of $h^{-}, p, \Lambda, \pi$, and $K_{S}^{0}$, Wenig [Wen90] was able to obtain an approximate energy balance between the incident bombarding energy and the energy detected in the final state. He estimated that not more than $\sim 70 \%$ of the final state energy is contained in charged hadrons and leading baryons which suggests that

$$
\varepsilon_{0} \gtrsim 1.5 \frac{\mathrm{GeV}}{\mathrm{fm}^{3}}
$$




\section{Chapter 7}

\section{Summary and Conclusions}

We have measured the transverse momentum spectra and rapidity distributions of negatively charged hadrons and protons from central ${ }^{32} S+{ }^{32} S$ interactions at 200 $\mathrm{GeV} /$ nucleon incident energy, the highest energy regime which has been explored in nucleus-nucleus collisions. The data were taken using the NA35 streamer chamber at the CERN SPS and a novel imaging and data acquisition system based on CCDs, which replaced film images with directly-digitized electronic pictures. The viability of this technique has been demonstrated. Because of our long-range objective of fully automating streamer chamber data reduction, we have analyzed our data in a completely different manner from that employed for the NA35 film measurements, relying heavily on Monte Carlo simulation to understand our acceptance, determine our efficiency and unfold the data. As a consequence, we have achieved an improvement in the data reduction rate and have obtained statistically equivalent or superior results from a smaller data sample. We have also carefully studied the influence that secondary charged particles, originating either from the weak decay of neutral strange particles or from hadronic interaction, could exert on the spectra and concluded that they can have a strong impact both on the transverse momentum distributions and on the proton rapidity distribution. We have corrected our data for these effects.

The negative hadron rapidity distribution is too broad to be accounted for by thermal models which demand isotropic emission, at any temperature. It is compatible with models which emphasize longitudinal dynamics, either phenomenological, string-breaking pictures, such as the Lund fragmentation model, which predict a plateau over several central units of rapidity or models which introduce one-dimensional, hydrodynamic expansion. The negative hadron data is not precise enough and our rapidity acceptance is not broad enough to distinguish between the Landau model, which assumes complete stopping and central particle production, and the symmetric Landau two-fireball model, which assumes nuclear transparency and particle production in the fragmentation regions.

We have advocated the latter point of view, based on the proton rapidity distribution which is rather flat but suggests a broad peak at $y \sim 1.5$. Although the two-fireball fit to the data shows only a small overlap between the projectile and target fragmentation regions, implying nuclear transparency, it is unlikely that the 
central region is baryon-free for the initial rapidity gap $\Delta y=6$ at incident nucleon momenta $p_{l a b}=200 \mathrm{GeV} / c$. There is no evidence for the sharp peak close to target rapidity predicted by $p p$ data, suggesting that nuclei are more effective in stopping the projectile than nucleon targets. The proton rapidity distribution indicates more stopping than the FRITIOF model of the superposition of pp collisions. However, the mean rapidity shift of the participant target protons is much smaller than that observed in central collisions of protons with heavy nuclei. It is unclear whether the high baryon densities predicted by extrapolations of central $p A$ results can be achieved even in the most central collisions of equal-mass nuclei. The mean rapidity shift suggests limited intranuclear cascading during the interpenetration phase of the collision. This interpretation is supported by the agreement between our estimate of the mean number of negative hadrons per participant nucleon-nucleon pair and the mean negative multiplicity from isospin-averaged, $N N$ collisions at comparable energy.

The negative hadron transverse momentum distribution is approximately independent of rapidity, suggesting that the invariant cross section factorizes into a product of functions which depend separately on $p_{T}$ and $y$. This is consistent with a dynamical picture which superimposes longitudinal expansion on a thermal equilibrium intermediate state. Although the negative hadron $p_{T}$ spectra possess a two-component structure, a comparison to pp data at comparable center-of-mass energy showed no evidence for enhanced production at low and high transverse momentum. Rather, the ratio of the $A A$ to $p p$ data appears to increase monotonically which may be evidence for rescattering in participant matter. The two-component structure can be explained by a thermal and chemical equilibrium model which takes into account the kinematics of resonance decay. For intermediate $p_{T}$, the transverse momentum distribution is sensitive to thermal pions and pions from $\rho$ meson decay and may provide a reasonable estimate of the underlying physical temperature. This interpretation is consistent with the proton $p_{T}$ distribution which has a single-component thermal structure, which is not altered by the presence of protons from $\Delta$ resonance decay. As demanded by a thermal model, the temperature determined from the proton distribution is the same as the high-temperature component of the negative hadron spectrum. Using an expression motivated by one-dimensional longitudinal expansion we estimate a freezeout temperature $T_{f} \sim 170 \mathrm{MeV}$. This temperature implies a much higher mean $p_{T}$ in nucleus-nucleus collisions than has been observed in $p p$ interactions, strong evidence for nucleon rescattering in participant matter. Combined with our conclusion of limited cascading during the interpenetration phase, the observation of thermal protons with high mean $p_{T}$ suggests that rescattering occurs during expansion.

The charged particle spectra of negative hadrons and protons can be accounted for in a coherent physical picture which assumes nuclear transparency, evolution through a state of thermal equilibrium, followed by longitudinal hydrodynamic expansion until freezeout. Depending on the timescale of the expansion, temperatures significantly higher than $T_{f}$ may have been reached earlier in the collision. Both the energy density and temperature in the high density stage of nucleus- 
nucleus collisions appear high enough to encourage optimism about the prospects for production of a quark-gluon plasma at SPS energies. 


\section{Bibliography}

[Abb90] T. Abbot et al., Phys. Rev. Lett. 64, 847 (1990)

[Abe88] K. Abe et al., Phys. Lett. 200B, 266 (1988)

[Ada87] M. Adamus et al., Phys. Lett. 198B, 427 (1987)

[Ake87] C.W. Akerlof et al., Nucl. Instrum. Methods A260, 80 (1987)

[Ake82] T. Åkesson et al., Nucl. Phys. B209, 309 (1982)

[Ake90] T. Åkesson et al., Z. Phys. C46, 361 (1990)

[Alb83] J.P. Albanese et al., Nucl. Instrum. Methods 212, 111 (1983)

[Alb88] R. Albrecht et al., Z. Phys. C38, 109 (1988)

[Alb90] R. Albrecht et al., Z. Phys. C47, 367 (1990)

[Ale90] T. Alexopoulos et al. Phys. Rev. Lett. 64, 991 (1990)

[Aln87] G.J Alner et al. ( UA5 Collaboration ), Phys. Rep. 154, 247 (1987)

[Alp75] B. Alper et. al., Nucl. Phys. B100, 237 (1975)

[Alp81] K. Alpgard et. al., Phys. Lett. 107B, 315 (1981)

[Alp82] K. Alpgard et al., Phys. Lett. 112B, 183 (1982)

[And83] B. Andersson et al., Phys. Rep. 97, 31 (1983)

[And87] B. Andersson, G. Gustafson and B. Nilsson-Almqvist, Nucl. Phys. B281, 289 (1987)

[Ani80] R. Anishetty, P. Koehler, and L. McLerran, Phys. Rev. D22, 2793 (1980)

[Ang88] S.A. Angius et al., Nucl. Instrum. Methods A273, 283 (1988)

[Art84] V. Artemiev et al., Nucl. Instrum. Methods 224, 408 (1984)

[Aus69] J.S. Auslander, Nucl. Instrum. Methods 74, 52 (1969)

[Aus76] J.S. Auslander, Nucl. Instrum. Methods 136, 319 (1976) 
[Bac91] J. Bächler et al., Z. Phys. C52, 239 (1991)

[Bal77] J. Ballam and R.D. Watt, Ann. Rev. Nucl. Sci. 27, 75 (1977)

[Bam87] A. Bamberger et al., Phys. Lett. 184B, 271 (1987)

[Bam88] A. Bamberger et al., Phys. Lett. 205B, 583 (1988)

[Bam89] A. Bamberger et al., Z. Phys. C43, 23 (1989)

[Bar90] J. Bartke et al., Z. Phys. C48, 191 (1990)

[Bar91] H.W. Barz, G. Bertsch, D. Kusnezov and H. Schulz, Phys. Lett. 254B, 332 (1991)

[Bas81] M. Basile et al., Phys. Scrip. 23:4, 743 (1981)

[Bay83] G. Raym et. al., Nucl. Phys. A407, 541 (1983)

[Bel85] W. Bell et al., Z. Phys. C27, 191 (1985)

[Bel86] W. Bell et al., Z. Phys. A325, 7 (1986)

[Ben69] J. Benecke et al., Phys. Rev. 188, 2159 (1969)

[Ben81] J.L. Benichou et al., Nucl. Instrum. Methods 190, 487 (1981)

[Bey80] J.D.E.Beynon and D.R. Lamb, Charge-Coupled Devices and their Applications, McGraw-Hill (1980)

[Bjo76] J.D. Bjorken, in Current Induced Reactions, Proceedings of the International Summer Institute on Theoretical Particle Physics, Hamburg, 1975, J.G. Körner, G. Kramer, and D. Schildknecht, eds., Springer-Verlag (1976)

[Bjo83] J.D. Bjorken, Phys. Rev. D27, 140 (1983)

[Blo74] V. Blobel et al., Nucl. Phys. B69, 454 (1974)

[Bla74] R. Blankenbecler and S.J. Brodsky, Phys. Rev. D10, 2973 (1974)

[Blo83] M.M Blourke et al., Opt. Eng. 22(5), 607 (1983)

[Blo85] M.M Blourke et al., "Large Area CCD Image Sensors for Scientific Applications", in Solid State Imaging Arrays, K.N. Prettyjohns and E.L. Dereniak, eds., Proc. SPIE 570, 82 (1985)

[Boc81] R. Bock, E. Pagiola and J. Zoll, HYDRA Topical Manual, CERN, Geneva (1981)

[Bog73] H. Bøggild et al., Nucl. Phys. B57, 77 (1973) 
[Bog74] H. Bøggild and T. Ferbel, Ann. Rev. Nucl. Sci. 24, 451 (1974)

[Bon78] J.P. Bondorf, S.I.A. Garpman, and J. Zimanyi, Nucl. Phys. A296, 320 (1978)

[Bow86] J.B. Bowlin and A.S. Goldhaber, Phys. Rev. D34, 778 (1986)

[Boy70] W.S. Boyle and G.E. Smith, Bell Syst. Tech. J. 49, 587 (1970)

[Bro84] R. Brockmann et al., Phys. Rev. Lett. 53, 2012 (1984)

[Bro91] G.E. Brown, J. Stachel, and G.M. Welke, Phys. Lett. 253B, 315 (1991)

[Bru74] F. Bruyant et al., HYDRA Application Library, CERN, Geneva (1974)

[Bru87] R. Brun et al., GEANT3 User's Guide, CERN, Geneva (1987)

[Bul67] F. Bulos et al., Slac Report SLAC-74 UC-28 TID-4500 (1967)

[Bun91] P. Buncic and A. Sandoval, GSI Report GSI-91-1 (1991)

[Bus84] W. Busza and A. Goldhaber, Phys. Lett. 139B, 235 (1984)

[Bus88] W. Busza and R. Ledoux, Ann. Rev. Nucl. Part. Sci. 38, 119 (1988)

[But60] J.C. Butcher and H. Messel, Nucl. Phys. 20, 15 (1960)

[Cad73] J.A. Cadzow, Discrete-Time Systems: an Introductory Approach with Interdisciplinary Applications, Prentice-Hall (1973)

[Cap80] A. Capella and J. Tran Thanh Van, Phys. Lett. 93B, 146 (1980)

[Cap81] A. Capella and J. Tran Thanh Van, Z. Phys. C10, 249 (1981)

[Car72] P. Carruthers and Minh Duong-van, Phys. Lett. 41B, 597 (1972)

[Car73] P. Carruthers and Minh Duong-van, Phys. Rev. D8, 859 (1973)

[Cha79] D. Chaney et a]., Phys. Rev. D19, 3210 (1979)

[Cha91] S. Chapman and M. Gyulassy, Phys. Rev. Lett. 67, 1210 (1991)

[Cha92] S.I. Chase, PhD thesis, University of California, Berkeley, in preparation.

[Cla86] R.B Clare and D. Strottman, Phys. Rep. 141, 177 (1986)

[Cle86] J. Cleymans et al., Phys. Rep. 130, 217 (1986)

[Col75] J.C. Collins and M.J. Perry, Phys. Rev. Lett. 34, 1353 (1975)

[Cos87] J. Costales, E802 internal memo, unpublished (1987)

[Cro69] M.H. Crowell and E.F. Labuda, Bell Syst, Tech. J. 48, 1481 (1969) 
[Cro75] J.W. Cronin et al., Phys. Rev. D11, 3105 (1975)

[Dam86] C.J.S. Damerell, "Vertex Detectors", in Techniques and Concepts of High-Energy Physics IV, edited by T. Ferbel, Plenum (1987)

[Dar88] P. Darriulat, Z. Phys. C38, 325 (1988)

[Das78] S. Das Gupta, Phys. Rev. Lett. 41, 1450 (1978)

[Das81] S. Das Gupta and A.Z. Mekjian, Phys. Rep. 72, 131 (1991)

[Dat85] S. Daté, M. Gyulassy, and H. Sumiyoshi, Phys. Rev. D32, 619 (1985)

[DeM82a] C. DeMarzo et al., Phys. Rev. D26, 1019 (1982)

[DeM82b] C. DeMarzo et al., Phys. Lett. 112B, 173 (1982)

[DeM83a] C. DeMarzo et al., Nucl. Instrum. Methods 217, 405 (1983)

[DeM83b] C. DeMarzo et al., Nucl. Phys. 211B, 375 (1983)

[DeM87a] C. DeMarzo et al., Phys. Rev. D36 8, (1987)

[DeM87b] C. DeMarzo et al., Phys. Rev. D36 16, (1987)

[Ead71] W.T. Eadie et al., Statistical Methods in Experimental Physics, pg 9, North Holland (1971)

[Eck73] V. Eckardt et al., Nucl. Phys. B55, 45 (1973)

[Eck77] V. Eckardt et al., Nucl. Instrum. Methods 143, 235 (1977)

[Eck78] V. Eckardt et al., Nucl. Instrum. Methods 155, 389 (1978)

[Eis79] Y. Eisenberg et al., Nucl. Phys. B154, 239 (1979)

[Eva67] D.A. Evans and W.H. Barkas, Nucl. Instrum. Methods 56, 289 (1967)

[Fae84] M.A. Faessler, Phys. Rep. 115, 1 (1984)

[Fer50] E. Fermi, Prog. Theor. Phys. 5, 570 (1950)

[Fer79] T. Ferbel, in Proceedings of the First Workshop on Ultra-Relativistic Nuclear Collisions, LBL Report LBL-8957 (1979)

[Fer84] T. Ferbel and W.R. Molzon, Rev. Mod. Phys. 56, 181 (1984)

[Fes85] H.C. Fesefeldt, PITHA Report PITHA-85-02 (1985)

[Fey69] R.P. Feynman, Phys. Rev. Lett. 23, 1415 (1969)

[Fin69] R.J. Finkelstein, Thermodynamics and Statistical Physics, W.H. Freeman (1969) 
[Fis73] C. M. Fisher, "Track Sensitive Targets and Rapid Cycling Bubble Chambers," in Proceedings of the 1973 International Conference on Instrumentation for High Energy Physics, edited by S. Stipcich, Frascati (1973)

[Fle89] B. Fleischmann, NA35 Internal Note, unpublished

[For78] R. Ford and W. Nelson, SLAC-210, UC-32 (1978)

[Fri73] D.C. Fries et al., Nucl. Instrum. Methods 107, 141 (1973)

[Fro79] A.G. Frodesen, O. Skjeggestad, and H. Töfte, Probability and Statistics in Particle Physics, Universitetsforlaget (1979)

[Fuc91] M. Fuchs et al., GSI Report GSI-91-1 (1991)

[Gar77] D. Garbut et al., Phys. Lett. 67B, 355 (1977)

[Gav91] S. Gavin and P.V. Ruuskanen, Phys. Lett. 262B, 326 (1991)

[Gaz87] M. Gazdzicki, D. Schall and K. Tittel, GSI Report GSI-87-1 (1987)

[Gaz91] M. Gazdzicki and O. Hansen, Nucl. Phys. A528, 754 (1991)

[Gel64] M. Gell-Mann, Phys. Lett. 8, 214 (1964)

[Gel82] R. Geller, in Proceedings of the Fourth Workshop on ECR Sources, Grenoble (1982)

[Ger89] H. von Gersdorff et al., Phys. Rev. C39, 1385 (1989)

[Ger90] P. Gerber, H. Leutwyler, and J.L. Goity, Phys. Lett. 246B, 513 (1990)

[Gia79] G. Giacomelli and M. Jacob, Phys. Rep. 55, 1 (1979)

[Glu63] R.L. Gluckstern, Nucl. Instrum. Methods 24, 381 (1963)

[Gol74] A.S. Goldhaber, Phys. Lett. 53B, 306 (1974)

[GSI85] NA35 Collaboration, GSI Report GSI-85-38 (1985)

[Gue76] K. Guettler et al., Phys. Lett. 64B, 111 (1976)

[Gyu84] M. Gyulassy and T. Matsui, Phys. Rev. D29, 419 (1984)

[Gyu87] M. Gyulassy, CERN-TH 4794/87 (1987)

[Gyu91] M. Gyulassy, private communication

[Hag65] R. Hagedorn, Suppl. Nuovo Cim. 3, 147 (1965)

[Hag68] R. Hagedorn and J. Ranft, Suppl. Nuovo Cim. 6, 169 (1968)

[Hag83] R. Hagedorn, Rev. Nuovo Cim. 6, no. 10 (1983) 
[Han88] M. Hansroul, H. Jeremie and D. Savard, Nucl. Instrum. Methods A270, 498 (1988)

[Har84a] J.W. Harris and R. Stock, LBL Report LBL-17054 (1984)

[Har84b] J.W. Harris et al., LBL Report LBL-19114 (1984)

[Har89] J.W. Harris, private communication

[Hec87] E. Hecht and A.Zajac, Optics, pg 230, Addison-Wesley (1987)

[Hec78] H. Heckman et al., Phys. Rev. C17, 1735 (1978)

[Hec88] W. Heck et al., Z. Phys. C38, 19 (1988)

[Hec90] W. Heck, private communication

[Hov89] L. Hovland, S. Geller and L. Acton, in Proceedings of the Second AIAA/JPL International Conference on Solar System Exploration, California Institute of Technology, Pasadena (1989)

[How79] M.J. Howes and D.V. Morgan, eds., Charge-Coupled Devices and Systems, Wiley (1979)

[Hyn79] J. Hyncek, IEEE IEDM Tech. Dig. 611 (1979)

[Ima67] K. Imaeda, Nuovo Cim. A48, 482 (1967)

[Ito70] N. Itoh, Prog. Theor. Phys. 44, 291 (1970)

[Jac72] M. Jacob and R. Slansky, Phys. Rev. D5, 1847 (1972)

[Jac91a] B. Jacak, Nucl. Phys. A525, 77c (1991)

[Jac91b] B. Jacak, private communication

[Jam80] F.E. James and M. Roos, Nucl. Phys. B172, 475 (1980)

[Jam89] F.E. James and M. Roos, MINUIT User's Guide, CERN, Geneva (1989)

[Jan77] G. Jansco et al., Nucl. Phys. B124, 1 (1977)

[Jan81] J.R. Janesick, J. Hynecek and M.M. Blourke, "Solid State Imagers for Astronomy", Proc. SPIE 290, 165 (1981)

[Jan85] J.R. Janesick et al., "The Future Scientific CCD", Proc. SPIE 501, 1 (1985)

[Jan90] J.R. Janesick, private communication

[Kad90] K. Kadija et al., LBL Report LBL-29651 (1990) 
[Kaf77] T. Kafka et al., Phys. Rev. D16, 1261 (1977)

[Kaj82] K. Kajantie and L. McLerran, Phys. Lett. 119B, 203 (1982)

[Kaj83a] K. Kajantie and L. McLerran, Nucl. Phys. B214, 261 (1983)

[Kaj83b] K. Kajantie, R. Raitio, and P.V. Ruuskanen, Nucl. Phys. B222, 152 (1983)

[Kat90] M. Kataja and P.V. Ruuskanen, Phys. Lett. 243B, 181 (1990)

[Kin78] K. Kinoshita, H. Satz, and D. Schildknecht, Phys. Rev D17, 1834 (1978)

[Kit89] T. Kitagaki et al., Nucl. Instrum. Methods A281, 81 (1989)

[Kla85] A. Klar and J. Hüffner, Phys. Rev. D31, 491 (1985)

[Kle86] K. Kleinknecht, Detectors for Particle Radiation, Cambridge University Press (1986)

[Kro90] D. Krofcheck et al., Nucl. Instrum. Methods A288, 497 (1990)

[Lan65] L.D. Landau, Collected Papers of L. D. Landau, edited by D. Ter Haar, Pergamon (1965), p. 569

[Lan69] L.D. Landau and E.M. Lifshitz, Course of Theoretical Physics Vol. 5, Statistical Physics, Pergamon (1969)

[Lau72] P Laurikainen, W.G. Moorhead and W. Matt, Nucl. Instrum. Methods 98, 349 (1972)

[Lee89] K.S. Lee and U. Heinz, Z. Phys. C43, 425 (1989)

[Lee90] K.S. Lee, U. Heinz and E. Schnedermann, Z. Phys. C48, 525 (1990)

[Lov91] W.A. Love et al., Nucl. Phys. A525, 601c (1991)

[Maj82] R. Majka et al., Nucl. Instrum. Methods 192, 241 (1982)

[Mar91] S. Margetis, GSI Report GSI-91-01 (1991)

[McL82] L. McLerran, Phys. Rep. 88, 379 (1982)

[McL86] L. McLerran, Rev. Mod. Phys. 58, 1021 (1986)

[Moi84] A.M. Moiseev, Sov. J. Part. Nucl. 15, 296 (1984)

[Mul85] B. Müller, The Physics of the Quark-Gluon Plasma, Springer-Verlag (1985)

[Nag82] S. Nagamiya, Phys. Rev. Lett. 49, 1383 (1982) 
[Nik81] N.N. Nikolaev, Sov. J. Part. Nucl. 12, 63 (1981)

[Nil86] B. Nilsson-Almqvist and E. Stenlund, University of Lund Report LU-TP 86-14 (1986)

[Ody90] G. Odyniec, private communication

[PDG88] Particle Data Group, G.P. Yost et al., Phys. Lett 204B, 1 (1988)

[Per74] M.L. Perl, High Energy Hadron Physics, Chapter 8, John Wiley \& Sons (1974)

[PQM88] Proc. Quark Matter 1987, 7. Phys. C38 (1988)

[PSC82] Proposal No. CERN/PSC/82, unpublished

[Pug86] H.G. Pugh et al., LBL Report LBL-22314 (1986)

[Pug90] H.G. Pugh, Phys. Scrip. T32, 208 (1990)

[Raj75] R. Raja, Nucl. Instrum. Methods 126, 247 (1975)

[Rem88] L.P. Remsburg, M.J. Tannenbaum and E802 collaboration, Z. Phys. C38, 35 (1988)

[Ren90] R. Renfordt, private communication

[Ric74] P. Rice-Evans, Spark, Streamer, Proportional and Drift Chambers, Richelieu Press Limited (1974)

[Roh87] D. Rohrich, PhD thesis, University of Marburg, unpublished

[Roh89] F. Rohrbach, NA35 Internal Note, unpublished

[San87] A. Sandoval et al., Nucl. Phys. A461 465, (1987)

[San91] A. Sandoval, private communication

[Sat85] H. Satz, Ann. Rev. Nucl. Part. Sci. 35, 245 (1985)

[Sch74] W. Scheid, H. Muller, and W. Greiner, Phys. Rev. Lett. 32, 741 (1974)

[Sey86] P. Seyboth, NA5 internal note

[Shu72] E.V. Shuryak, Yad. Fiz. 16, 395 (1972)

[Sie79] P.J. Siemens and J.O. Rasmussen, Phys. Rev. Lett. 42, 880 (1979)

[Sjo86] T. Sjostrand, Computer Phys. Comm. 39, 347 (1986)

[Sol65] F. Solmitz et al., Three View Geometry Program - TVGP, Alvarez Group Progr. Note, P-117 LRL 
[Sol90] J. Sollfrank, P. Koch and U. Heinz, Phys. Lett. 252B, 256 (1990)

[Soo67] K. Soop, "Bubble Chamber Measurements and Their Evaluation", in Progress in Nuclear Techniques and Instrumentation Volume 2, North Holland (1967)

[SPS75] Proposal No. CERN/SPSC/75-1/P37, unpublished

[Sta89a] J. Stachel and P. Braun-Munzinger, Phys. Lett. 216B, 1 (1989)

[Sta89b] J. Stachel and P. Braun-Munzinger, Nucl. Phys. A495, 393c (1989)

[Sta91] J. Stachel, Nucl. Phys. A527, 167c (1991)

[Sto86] R. Stock, Phys. Rep. 135, 259 (1986)

[Str84] H. Stroebele, Nucl. Instrum. Methods 221, 523 (1984)

[Str88] H. Stroebele et al., Z. Phys. C38, 89 (1988)

[Str91] H. Stroebele, private communication

[Tho77] W. Thome et al., Nucl. Phys. B129, 365 (1977).

[Tin87] M.L. Tincknell et al., Opt. Eng. 26, 1067 (1987)

[To087] W.S. Toothacker et al., Phys. Lett. 197B, 295 (1987)

[Van66] J.T. Vanderslice, H.W. Schamp, Jr., and E.A. Mason, Thermodynamics, Prentice-Hall (1966)

[Wen90] S. Wenig, GSI-Report GSI-90-23 (1990)

[Wes76] G.D. Westfall et al., Phys. Rev. Lett. 37, 1202 (1976)

[Whi74] J. Whitmore, Phys. Rep. 10, 273 (1974)

[Wol86] B.H. Wolf et al., GSI-Report GSI-86-02 (1986)

[Wol91] K. Wolf, private communication

[Wro85] A. Wróblewski, Acta Phys. Polonica B16, 379 (1985)

[Zal85] K. Zalewski, Ann. Rev. Nucl. Part. Sci. 35, 55 (1985) 

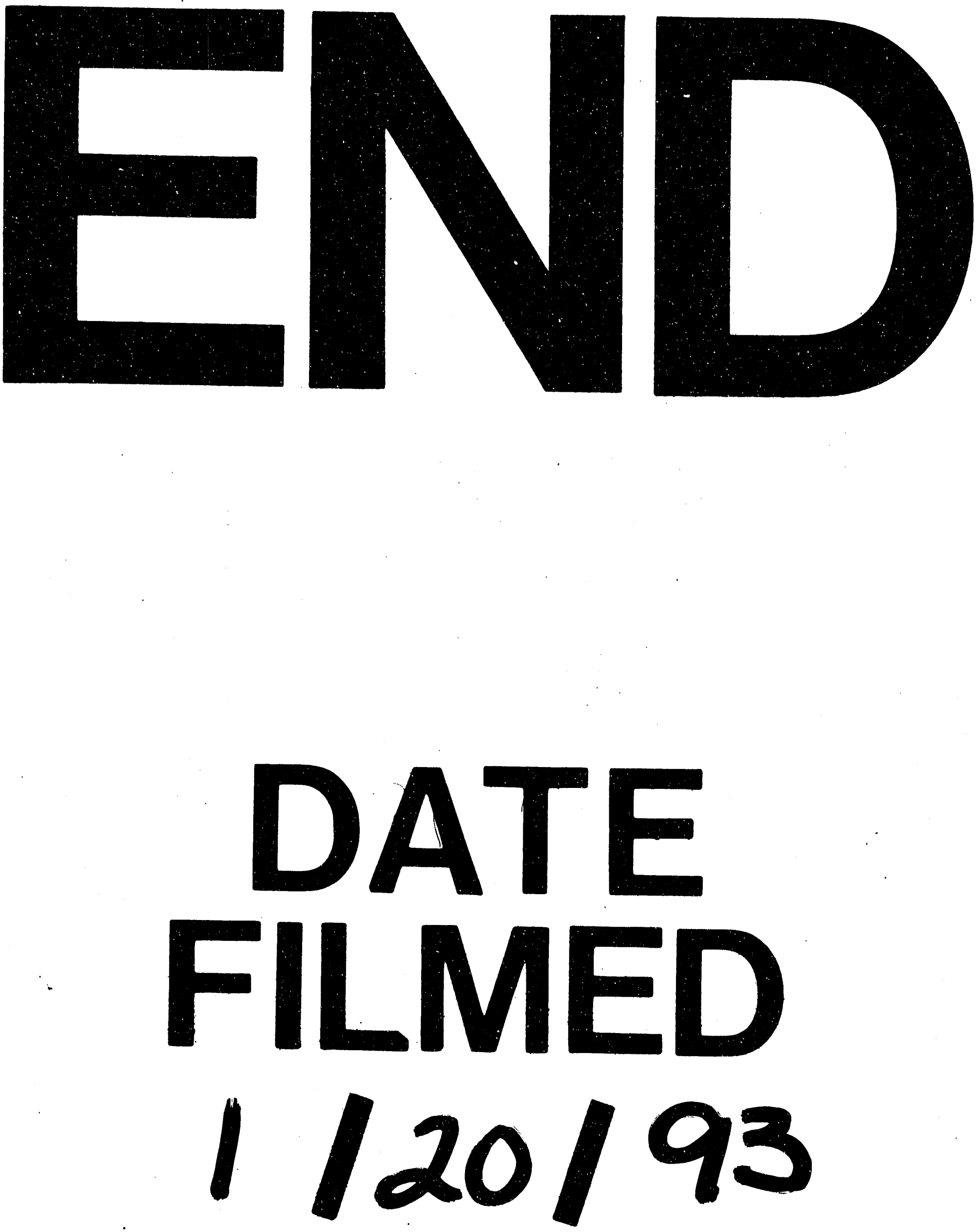
